\title{
The Impact of the Perceived Value of Records on the Use of Electronic Recordkeeping Systems
}

\author{
by \\ Matthew James Lewellen
}

A thesis

submitted to the Victoria University of Wellington in fulfilment of the requirements for the degree of Doctor of Philosophy

Victoria University of Wellington

2015 



\begin{abstract}
Today's electronic documents and digital records are rapidly superseding traditional paper records and similarly need to be managed and stored for the future. This need is driving new theoretical recordkeeping models, international electronic recordkeeping standards, many instances of national recordkeeping legislation, and the rapid development of electronic recordkeeping systems for use in organizations. Given the legislative imperative, the exponential growth of electronic records, and the importance to the individual, organization, and society of trustworthy electronic recordkeeping, the question arises: why are electronic recordkeeping systems experiencing different rates of acceptance and utilization by end users? This research seeks to address that question through identifying the factors that influence a user's intention to use an electronic recordkeeping system.

Although a significant body of research has been dedicated to studying system use in various situations, no research in the information systems discipline has yet focused specifically on electronic recordkeeping and its unique set of use-influencing factors.

This research creates a new conceptual research model by selecting constructs to represent the technology acceptance literature and adding additional constructs to represent organizational context and knowledge interpretation. It also introduces a new construct: the perceived value of records.

A survey instrument was developed and administered to a sample of public servants from the New Zealand government in order to evaluate the research model quantitatively and determine the relative importance of the factors.

By identifying the factors that impact the use of electronic recordkeeping systems, this research will inform future strategies to improve the capture and retention of our digital heritage. As Archives New Zealand states: "Do nothing, lose everything. If no action is taken, public sector digital information will be lost."
\end{abstract}




\section{Acknowledgments}

I wish to express my sincere thanks and appreciation to the following people and organizations who have supported me in this project:

- My primary supervisor, Val Hooper, for her rigorous attention to detail, continuous encouragement, insightful suggestions and guidance, and the time taken to read this tome across numerous time zones.

- My co-supervisor, Gillian Oliver, for her insight, guidance, enthusiasm, kind words, encouragement, and contagious love of recordkeeping.

- The New Zealand public sector people and organizations who supported me with their time, access, and willingness to contribute to this research.

- My friend Thoron Hollard, who helped me sort out my en dashes and semicolons, because he really cares about that sort of thing.

- To my wife, Ruth, who willingly agreed to take up many of my family responsibilities to allow me to carve out research time, encouraged me to work hard and stay on schedule even when I didn't want to, and provided the rock-solid foundation on which this project was built (... even though she still refuses to read the final output).

- And to my boys, Keiran and Brayden, for their love of learning and constant willingness to provide distraction. 


\section{Contents}

1 Introduction $\quad 1$

1.1 Chapter Overview . . . . . . . . . . . . . . . . . . . 1

1.2 Motivation for the Research . . . . . . . . . . . . . . . . . 1

1.3 Research Gap . . . . . . . . . . . . . . . . . . . 3

1.4 Research Questions and Objectives ... . . . . . . . . 4

1.5 Research Methodology . . . . . . . . . . . . . . 5

1.6 Value and Importance of the Research . . . . . . . . . . 5

1.6.1 Academic Value of the Research . . . . . . . . . . . 6

1.6.2 Practitioner Value of the Research . . . . . . . . . 6

1.7 Thesis Outline . . . . . . . . . . . . . . . . 7

1.8 Chapter Summary . . . . . . . . . . . . . . . 8

2 Literature Review $\quad 9$

2.1 Chapter Overview . . . . . . . . . . . . . . . . . . . . . . 9

2.2 Records and Recordkeeping . . . . . . . . . . . . . . . . 10

2.2.1 Key Characteristics of Records . . . . . . . . . . 11

2.2.2 A Short History of Records and Recordkeeping . . . . . 14

2.2.3 A Short History of Electronic Recordkeeping . . . . . . 16

2.3 Recordkeeping Models and Theories . . . . . . . . . . . . . . 19

2.3.1 Structuration Theory . . . . . . . . . . . . . . . . 19

2.3.2 The Records Continuum Model . . . . . . . . . . . . 21

2.4 Electronic Document and Records Management . . . . . . . . 25

2.4.1 Reorganizing Recordkeeping . . . . . . . . . . . . 25

2.4.2 EDRMS Defined . . . . . . . . . . . . . 27

2.4.3 The Future of EDRMS . . . . . . . . . . . . . . . 28

2.5 EDRMS-Use Constructs . . . . . . . . . . . . . . . . . . 30

2.5.1 System Use: the Dependent Variable . . . . . . . . . . 30

2.5.2 Determinants of System Use . . . . . . . . . . . . 35

2.6 Chapter Summary . . . . . . . . . . . . . . . 55 
3 Conceptual Research Model $\quad 57$

3.1 Chapter Overview . . . . . . . . . . . . . . . . 57

3.2 Technology Acceptance Factors . . . . . . . . . . . . . 58

3.2.1 Effort Expectancy . . . . . . . . . . . . . . 59

3.2.2 Performance Expectancy . . . . . . . . . . . . . . . 59

3.3 Organizational Context Factors . . . . . . . . . . . . . 60

3.3 .1 Social Influence . . . . . . . . . . . . . . . 60

3.3.2 Perceived Power Security . . . . . . . . . . . . 61

3.4 Knowledge Interpretation . . . . . . . . . . . . . . . 62

3.5 Chapter Summary . . . . . . . . . . . . . . . 64

4 Research Design and Methodology $\quad 67$

4.1 Chapter Overview . . . . . . . . . . . . . . . . . . . 67

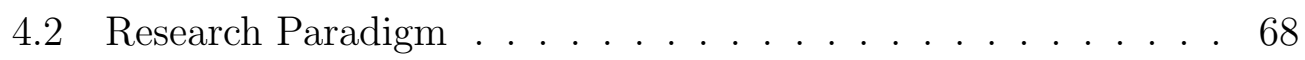

4.3 Sample Population . . . . . . . . . . . . . . . . . 70

4.4 Research Methodology . . . . . . . . . . . . . . . . 71

4.4.1 Phase I: Qualitative Methods . . . . . . . . . . . 73

4.4.2 Phase II: Quantitative Methods . . . . . . . . . . 76

4.5 Chapter Summary . . . . . . . . . . . . . . 80

5 Phase I: Qualitative Methods $\quad 81$

5.1 Chapter Overview . . . . . . . . . . . . . . . . . . . 81

5.2 Stage 1: Interviews . . . . . . . . . . . . . . . . . 82

5.2 .1 Interview Instrument Creation . . . . . . . . . . . . 82

5.2 .2 Data Collection Methods . . . . . . . . . . . . 83

5.2.3 Thematic Analysis Methods . . . . . . . . . . . . 84

5.2.4 Measurement Item Creation Analysis and Results . . . 86

5.3 Stage 2: Proofreading . . . . . . . . . . . . . 107

5.3.1 Proofreading Methods . . . . . . . . . . . 107

5.3.2 Proofreading Analysis and Results . . . . . . . . . 108

5.4 Stage 3: Card Sorting . . . . . . . . . . . . . . . 112

5.4.1 Card Sorting Introduction Procedure . . . . . . . . . . 113

5.4 .2 Closed Card Sorting Methods . . . . . . . . . . . . . 115

5.4 .3 Open Card Sorting Methods . . . . . . . . . . . . . . 116

5.4.4 Card Sorting Analysis and Results . . . . . . . . . . 117

5.5 Chapter Summary . . . . . . . . . . . . . . . . . . . 126 
6 Phase II: Quantitative Methods 129

6.1 Chapter Overview . . . . . . . . . . . . . . . . . . . . . . . . . . . 129

6.2 Stage 4: Survey Design . . . . . . . . . . . . . . . . 130

6.2.1 Online Survey Platform . . . . . . . . . . . . . 130

6.2.2 Analysis-Informed Data Capture . . . . . . . . . . . 131

6.2.3 Creation of Survey Instrument . . . . . . . . . . . . . . 132

6.2.4 Survey Documentation Templates . . . . . . . . . . 133

6.3 Stage 5: Survey Pretest . . . . . . . . . . . . . . . 133

6.4 Stage 6: Survey . . . . . . . . . . . . . . . . . . . 135

6.5 Chapter Summary . . . . . . . . . . . . . . . 135

7 Phase II: Data Analysis 137

7.1 Chapter Overview . . . . . . . . . . . . . . . . . . . . . . 137

7.2 Descriptive Statistics . . . . . . . . . . . . . . . 138

7.2.1 Response Rate . . . . . . . . . . . . . . . . . . . . . 138

7.2.2 Sample Demographics . . . . . . . . . . . . . . . 139

7.2.3 EDRMS Experience Profile . . . . . . . . . . . . . 141

7.3 Factor Analysis . . . . . . . . . . . . . . . . . . . . . . . . . 143

7.3.1 Circle of Correlations . . . . . . . . . . . . . . . . 144

7.3.2 Factor Analysis by Construct . . . . . . . . . . . . . . 145

7.4 Partial Least Squares Path Modeling (PLS-PM) . . . . . . . . 162

7.4.1 Selection of PLS-PM . . . . . . . . . . . . . 162

7.4.2 PLS-PM Described . . . . . . . . . . . . . . . . 164

7.4.3 PLS-PM Outer (Measurement) Model Analysis . . . . 165

7.4.4 PLS-PM Inner (Structural) Model Analysis . . . . . . 172

7.4.5 PLS-PM Model Significance Tests . . . . . . . . . . . . 175

7.4.6 PLS-PM Bootstrapping and Model Validation . . . . . 179

7.5 Analysis of Hypothesized Effects . . . . . . . . . . . . . . . . . 181

7.6 Additional Comments by Respondents . . . . . . . . . . . . . 184

7.7 Chapter Summary . . . . . . . . . . . . . . . . . 188

8 Discussion $r 191$

8.1 Chapter Overview . . . . . . . . . . . . . . . . . . . . 191

8.2 The Measures of the Constructs . . . . . . . . . . . . . . . . . 192

8.2.1 Technology Acceptance . . . . . . . . . . . . . . . 192

8.2.2 Organizational Context . . . . . . . . . . . . . 195

8.2.3 Knowledge Interpretation . . . . . . . . . . . . . . . 199

8.3 The Research Model . . . . . . . . . . . . . . . . . . . . . . 202

8.3.1 Hypothesized Path Effects . . . . . . . . . . . . 203 
8.3.2 The Explanatory Power of the Model . . . . . . . . . . 212

8.4 Chapter Summary . . . . . . . . . . . . . . . . . . . 212

9 Conclusions $\quad 215$

9.1 Chapter Overview . . . . . . . . . . . . . . . . . . . . . 215

9.2 Overview of the Research . . . . . . . . . . . . . . . . . 216

9.2.1 Research Gap . . . . . . . . . . . . . . . . . 216

9.2.2 Research Objectives and Conceptual Model . . . . . . 216

9.2.3 Research Design . . . . . . . . . . . . . . . . . . 218

9.2.4 Research Findings and Model Evaluation . . . . . . . . 220

9.3 Contributions of the Research . . . . . . . . . . . . . . . . . . 221

9.3.1 Academic Value of the Research . . . . . . . . . . . . . 221

9.3.2 Practitioner Value of the Research . . . . . . . . . . . . 223

9.3.3 Implications of the Research . . . . . . . . . . . . . . 223

9.4 Limitations of the Research . . . . . . . . . . . . . . . 224

9.4.1 Limitations of the Conceptual Model . . . . . . . . . . 224

9.4.2 Limitations of the Research Design . . . . . . . . . . 225

9.4.3 Limitations of the Research Instrument . . . . . . . . . 226

9.5 Directions for Future Research . . . . . . . . . . . . . . . . 227

9.5.1 Improved Measures of Social Influence . . . . . . . . . 227

9.5.2 Social Influence in Mandatory Use Situations . . . . . 228

9.5.3 Aligment with the Records Continuum Model . . . . . 229

9.5.4 Development of a Multilevel Theoretical Model . . . . 229

9.5.5 Benchmarking the Prevalence of the Four Knowledge

Management Discourses . . . . . . . . . . . . 230

9.6 Chapter Summary . . . . . . . . . . . . . . . . . . . . . . 230

$\begin{array}{ll}\text { A Interview Instrument } & 231\end{array}$

B Proofreading of Stage 1 Items 235

C Final Survey Instrument $\quad 239$

D Survey Communications Templates 249

$\begin{array}{ll}\text { E Final Survey Data } & 257\end{array}$

F Bootstrap Validation $\quad 265$ 


\section{List of Figures}

2.1 Conceptual model of recordkeeping metadata (Final Report CRKM Project, 2007) . . . . . . . . . . . . . . . 18

2.2 Dimensions of the duality of structure (Giddens, 1984) . . . . 20

2.3 The Records Continuum Model (Upward, 2005) . . . . . . . . 24

2.4 Example of a recordkeeping metadata relationship (McKemmish, Acland, Ward, \& Reed, 1999) . . . . . . . . . . 26

2.5 'IS use' from variance and process perspectives (Fidock \& Carroll, 2009) . . . . . . . . . . . . . . . . . 31

2.6 DeLone and McLean IS Success Model (DeLone \& McLean, 1992) . . . . . . . . . . . . . . . . . . . . . 32

2.7 Seddon's respecification of the DeLone \& McLean IS Success Model (Seddon, 1997) . . . . . . . . . . . . . . 33

2.8 Past conceptualizations of the system-usage construct (BurtonJones \& Straub, 2006) . . . . . . . . . . . . . . 34

2.9 TAM: Technology Acceptance Model (Davis, 1986) . . . . . . 36

2.10 TAM2: extension of the Technology Acceptance Model (Venkatesh \& Davis, 2000) . . . . . . . . . . . . . . . . . 39

2.11 UTAUT: Unified Theory of Acceptance and Use of Technology (Venkatesh, Morris, Davis, \& Davis, 2003) . . . . . . . . . . . 41

2.12 TAM3: Technology Acceptance Model 3 (Venkatesh \& Bala, 2008) . . . . . . . . . . . . . . . . . 43

2.13 TRA: Theory of Reasoned Action (Davis, Bagozzi, \& Warshaw, 1989) . . . . . . . . . . . . . . . 4 46

2.14 Addition of the subjective norm to the Technology Acceptance Model (Schepers \& Wetzels, 2007) . . . . . . . . . . . . 47

2.15 Addition of perceived power security and the subjective norm to TAM (Ong, Lai, Wang, \& Wang, 2005) . . . . . . . . . 49

2.16 Four discourses of knowledge management research (Schultze \& Stabell, 2004) . . . . . . . . . . . . . 52 
3.1 Conceptual Research Model of EDRMS Use . . . . . . . . . 58

3.2 Research Model of EDRMS-use . . . . . . . . . . . . . . 65

5.1 Card sorting data: example of raw data capture . . . . . . . 117

5.2 Dendrogram of closed card sort data (unadjusted) . . . . . . . 118

5.3 Dendrogram of closed card sort data (adjusted) . . . . . . . . 124

5.4 Dendrogram of open card sort data (unadjusted) . . . . . . . . 125

5.5 Dendrogram of open card sort data (adjusted) . . . . . . . . 126

7.1 Survey Participant Demographics . . . . . . . . . . . . . . . 140

7.2 Survey: EDRMS Experience Profile . . . . . . . . . . . . . . . 142

7.3 Circle of Correlations: Effort Expectancy . . . . . . . . . . . . 146

7.4 Circle of Correlations: Performance Expectancy . . . . . . . . 149

7.5 Circle of Correlations: Social Influence . . . . . . . . . . . . . 152

7.6 Circle of Correlations: Perceived Power Security . . . . . . . . 155

7.7 Circle of Correlations: Social Influence and Power measurement items . . . . . . . . . . . . . . 156

7.8 Circle of Correlations: Power-related measurement items . . . 157

7.9 Circle of Correlations: Perceived Value of Records . . . . . . . 159

7.10 Plot: Outer Model Loadings . . . . . . . . . . . . . . . . 167

7.11 Bar chart: measurement item loadings . . . . . . . . . . . 168

7.12 Research Model with Path Coefficients . . . . . . . . . . . . . 173

7.13 Construct Correlation Matrix . . . . . . . . . . . . . . 178

7.14 The Measured Research Model . . . . . . . . . . . . . . . . . 181

8.1 Bar chart of Path Effects . . . . . . . . . . . . . 203

C.1 Survey Instrument (Page 1) Consent to Participate . . . . . . 240

C.2 Survey Instrument (Page 2) Effort Expectancy . . . . . . . . . 241

C.3 Survey Instrument (Page 3) Performance Expectancy . . . . . 242

C.4 Survey Instrument (Page 4) Social Influence . . . . . . . . . . 243

C.5 Survey Instrument (Page 5) Perceived Power Security and Perceived Value of Records . . . . . . . . . . . . . . . . . 244

C.6 Survey Instrument (Page 6) Attitude and Intention-to-use . . 245

C.7 Survey Instrument (Page 7) Demographics . . . . . . . . . . . 246

C.8 Survey Instrument (Page 8) Thank You confirmation . . . . . 247

D.1 Research Information Sheet . . . . . . . . . . . . . . 252

E.1 Bar Chart: Effort Expectancy survey responses . . . . . . . . . 258 
E.2 Bar Chart: Performance Expectancy survey responses . . . . . 259

E.3 Bar Chart: Social Influence survey responses . . . . . . . . . . 260

E.4 Bar Chart: Perceived Power Security survey responses . . . . 261

E.5 Bar Chart: Perceived Value of Records survey responses . . . . 262

E.6 Bar Chart: Intention to Use survey responses . . . . . . . . . 263 


\section{List of Tables}

5.1 Phases of Thematic Analysis (Braun \& Clarke, 2006) . . . . . 85

5.2 Performance Expectancy: thematic analysis . . . . . . . . 87

5.3 Performance Expectancy: items from the literature . . . . . . 88

5.4 Performance Expectancy: items from Stage 1 . . . . . . . . . . 90

5.5 Effort Expectancy: thematic analysis . . . . . . . . . . . . 91

5.6 Effort Expectancy: items from the literature . . . . . . . . . . 92

5.7 Effort Expectancy: items from Stage 1 . . . . . . . . . . 93

5.8 Social Influence: thematic analysis . . . . . . . . . . . . . . . 94

5.9 Social Influence: items from the literature . . . . . . . . . . 95

5.10 Social Influence: items from Stage 1 . . . . . . . . . . . . 97

5.11 Perceived Power Security: thematic analysis . . . . . . . . . . 99

5.12 Perceived Power Security: items from the literature . . . . . . 99

5.13 Perceived Power Security: items from Stage 1 . . . . . . . . . 100

5.14 Perceived Value of Records: thematic analysis . . . . . . . . . 101

5.15 Perceived Value of Records: items from Stage 1 . . . . . . . . 105

5.16 Proofreading: distribution of proofreading feedback . . . . . . 108

5.17 Measurement Items: items from Stage 2 . . . . . . . . . . 111

5.18 Closed card sort: agreement frequency matrix (unadjusted) . . 119

5.19 Closed card sort: agreement frequency matrix (adjusted) . . . 123

7.1 Effort Expectancy (EE): PCA-based item pool . . . . . . . . . 147

7.2 Performance Expectancy (PE): PCA-based item pool . . . . . 150

7.3 Social Influence (SI): PCA-based item pool . . . . . . . . . . . 153

7.4 Perceived Value of Records (PVR): PCA-based item pool . . . 160

7.5 Outer Model Measures of Fit. . . . . . . . . . . . . . . . . . 166

7.6 Unidimensionality Indicators: measures of validity . . . . . . . 169

7.7 Inter-construct correlations and reliability measures . . . . . . 170

7.8 Outer Model Loadings and Cross-loadings . . . . . . . . . . . 171

7.9 Total Path Effects . . . . . . . . . . . . . . . . . . . . . . 174 
7.10 Inner Model Significance Tests . . . . . . . . . . . . . . 176

7.11 Measures of Quality of Model Fit . . . . . . . . . . . . . . 179

7.12 Research Hypotheses Results Summary . . . . . . . . . . . . . 182

7.13 Survey Open Answer Question: thematic analysis . . . . . . . 185

8.1 Belief that recordkeeping is mandatory . . . . . . . . . . 196

8.2 Perceived Value of Records 2x2 Value Matrix . . . . . . . . . . 202

F.1 Bootstrapping Analysis: Weights . . . . . . . . . . . . 266

F.2 Bootstrapping Analysis: Loadings . . . . . . . . . . . . . . . . 267

F.3 Bootstrapping Analysis: Paths . . . . . . . . . . . . . . . . . . 268

F.4 Bootstrapping Analysis: R-squared . . . . . . . . . . . . 268 


\section{Chapter 1}

\section{Introduction}

\section{Contents}

1.1 Chapter Overview . . . . . . . . . . . 1

1.2 Motivation for the Research . . . . . . . . . 1

1.3 Research Gap . . . . . . . . . . . . . 3

1.4 Research Questions and Objectives ....... 4

1.5 Research Methodology . . . . . . . . . . 5

1.6 Value and Importance of the Research . . . . 5

1.7 Thesis Outline . . . . . . . . . . . 7

1.8 Chapter Summary . . . . . . . . . . 8

\subsection{Chapter Overview}

This chapter provides an introduction to the thesis and explains the motivation for the research undertaken. The central research question and objectives are set out, and the methodological approach is summarized. The chapter ends with an outline of subsequent chapters.

\subsection{Motivation for the Research}

Records and archives have been at the foundation of human civilization since ancient times. The word archive (Greek: archeion) describes the 'keystone' of an arch. Metaphorically, the ancient Greeks considered the archive as the 
keystone in the arch of government - the crucial piece that keeps the arch from crumbling; thereby underlining the importance of records and archives to society. They recognized the importance of reliable recorded evidence to the functioning of government and created a special location to store that evidence (Upward, 2005).

In modern times, the ability of an organization to collect, store, and use knowledge (in the form of records) has important consequences for its performance (Olivera, 2000). However, records must be trustworthy for an organization to rely on them to meet its legal and business requirements (NARA, 2005; Cullen, Hirtle, Levy, Lynch, \& Rothenberg, 2000). To meet those requirements, a specialist discipline - records management - developed over time to support the collection, classification, storage, and retrieval of records. This discipline also addresses methods to manage the key characteristics of records: reliability, authenticity, integrity, and usability (ISO 15489.1, 2002). Traditionally, this discipline has focused on physical (paper) records.

At the beginning of the twenty-first century, governments in many countries began to implement electronic document and records management systems EDRMS (Nguyen, Swatman, \& Fraunholz, 2009; Agourram \& Robson, 2006; Johnston \& Bowen, 2005; Sprehe, 2005). This is often done in an attempt to manage the exponential increase in both electronic records (Duranti, 2010) and the "unstructured information captured in paper and electronic formats, such as emails, word processed and spreadsheet contents" (Joseph, 2008) produced by end users. EDRMS are also implemented in response to legislative imperatives such as the New Zealand Public Records Act (2005) or the US Sarbanes-Oxley Act of 2002 (Nguyen \& Swatman, 2007). Recordkeeping legislation has raised the profile of organizational recordkeeping standards and their importance at the regulatory level.

EDRMS are being used to improve and control electronic recordkeeping within organizations (Svärd, 2013; Wilkins, Swatman, \& Holt, 2009a; Joseph, 2008). An important implication is that these systems transfer the responsibility of recordkeeping from the records and information management (RIM) professionals to the end users, those prolific creators of the electronic records (Joseph, Debowski, \& Goldschmidt, 2012). Consequently, end users now have responsibility for both identifying documents worthy of retention and applying descriptive metadata to those records. The selection of descriptive metadata (including the correct application of suitable records classifications) drives the application of retention schedules and ultimately impacts future contextual search and retrieval (Joseph, Debowski, \& Goldschmidt, 2013; Sprehe \& McClure, 2005). 
In spite of the active interest by senior management in investing in largescale implementations of EDRMS, multiple published case studies observed that rates of acceptance and use of these systems by end users were often disappointing (S. Jones, 2012; Nguyen et al., 2009; Maguire, 2005; Smyth, 2005; D. Williams, 2005). Low rates of use could lead to an unproductive cycle where users who chose not to store records in the system are not likely to use the system to retrieve records since it is unlikely that the records they were seeking would have been stored in the system initially. ${ }^{1}$

Nonetheless, there are also examples of successful EDRMS implementations with high utilization and tangible benefits (Wilkins, Swatman, \& Holt, 2009b, 2007; Nguyen, Swatman, \& Fraunholz, 2008a; D. Williams, 2005).

By identifying the factors that impact the acceptance and use of electronic recordkeeping systems, this research will inform future strategies to improve the capture and retention of our digital heritage. An electronic document can only be a trustworthy record if it is managed systematically (the primary purpose of the EDRMS). As Archives New Zealand states: "Do nothing, lose everything. If no action is taken, public sector digital information will be lost" (Archives New Zealand, 2009, p. 8).

\subsection{Research Gap}

Electronic recordkeeping systems that rely on end user participation are a useful class of system for studying a user's intention to use a system. Even though the use of such systems can be mandated by senior management or even by legislation, the actual use of the systems requires action and judgment by end users: action, in appropriately identifying records; and judgment, in storing such records in logical contextual categories and classifications. As such, the electronic recordkeeping environment provides a significant margin for users to participate or not according to their perception of the importance of their documents and the information they contain.

\footnotetext{
${ }^{1}$ This low utilization reflects the capture of comparatively simple office-style documents. More complex records - particularly those forming in Web 2.0 technologies - are experiencing their own set of recordkeeping challenges (Lips \& Rapson, 2009, 2010). From a records perspective, the impact of low utilization also means that documents are not being properly managed, and can be lost through destruction or deterioration, or else made irretrievable through lack of an indexing mechanism (Wilson, 1995). Loss of these records also has an impact on society. In the short term, the loss of accountability - a cornerstone of our legal system and civilization, and in the long term, ultimately a loss of history (Johnston \& Bowen, 2005).
} 
The reasons that explain and support utilization rates of generic Information Systems (IS) have been widely studied and have been organized into a variety of well-tested and validated technology acceptance models. Leading examples of such measurement models include Davis's (1989) Technology Acceptance Model (TAM); Goodhue \& Thompson's (1995) Task-Technology Fit (TTF) model; Dishaw and Strong's (1999) combined TAM/TTF model; Venkatesh and Davis's (2000) extended TAM2 model; Venkatesh, Morris, Davis, \& Davis's (2003) Unified Theory of Acceptance and Use of Technology (UTAUT) model; and Venkatesh and Bala's (2008) Technology Acceptance Model 3 (TAM3).

Acknowledging the known limitations of these models (Benbasat \& Barki, 2007), they have nonetheless been successfully applied to a wide variety of traditional IS tools and systems, albeit with few examples having been applied to any information management-class systems. Some limited efforts have been made to apply these models to classes of systems that are similar to that of this research, including Organizational Memory Systems (Li, Baowei, \& Ye-zhuang, 2007, 2004) and Knowledge Management Systems (Li et al., 2007), which primarily focus on capturing 'lessons learned' to improve future performance. However, no studies have been found to date that apply technology acceptance-class models directly to electronic recordkeeping systems with their unique set of contexts and use cases. Furthermore, the models, when applied in these related studies, have shown variable and differing results in explaining system utilization rates.

\subsection{Research Questions and Objectives}

The main research question considered is:

- What factors influence a user's intention to use an electronic recordkeeping system?

Converted into the research objectives, the aim of the research is:

- To identify the factors that influence a user's intention to use an electronic recordkeeping system.

- To determine the relative importance of the factors that influence a user's intention to use an electronic recordkeeping system. 


\subsection{Research Methodology}

As the factors that influence a user's intention to use an electronic recordkeeping system are not known, the research began with a literature review. This review revealed the importance of records and recordkeeping in society, introduced the underlying theories and models that support modern recordkeeping practices and standards, and introduced prior research that focused on similar aspects of technology acceptance, albeit not within a recordkeeping context.

Taking a post-positivist stance and a reductionist approach (Creswell, 2008), a conceptual research model was then created based on the findings from the literature review. Most of the constructs required by the model were already available and validated; however, there was one aspect that was incomplete. This required the development of a new construct to complete the model: the perceived value of records. The research then turned to the construct measurement items to determine if they (and the constructs themselves) were sufficient to be used within a recordkeeping context.

A mixed-method approach was adopted consisting first of a qualitative phase to explore and seek out suitable contextually-appropriate measurement items that adequately reflect the conceptual model's constructs. To this end, data were collected via personal interviews and a pool of measurement items was developed based on an analysis of the interview transcripts. Additional data-gathering activities were used to further assign and validate the measurement-item-to-construct relationships, with the knowledge that these measurement items would eventually be used to construct the quantitative survey instrument.

In the quantitative phase, the survey instrument (consisting primarily of the construct measurement items) was developed to gather data for the measurement and validation of the research model. The data were collected via an online survey of a large New Zealand public sector organization.

\subsection{Value and Importance of the Research}

This research provided the first empirical measurement of the set of unique acceptance and use factors associated with electronic recordkeeping systems. The main benefits of the research to academics and practioners are introduced below. 


\subsubsection{Academic Value of the Research}

The primary academic contribution of this research is the combination of existing IS technology acceptance theory with recordkeeping and its set of underlying theories.

Information systems that are designed to support recordkeeping requirements are quite complicated, and they require the input of extensive recordkeeping metadata and an understanding of what records are and how records should be stored and organized. Since electronic recordkeeping systems expect end users to take on this responsibility, these systems end up inhabiting a unique position in the list of enterprise information systems in that the use of these systems is often ancillary to the end user's main roles, responsibilities, and performance measures.

To address this gap, this research develops a new construct: the perceived value of records. This new construct is defined, provided with a set of measurement items, and empirically measured and validated.

Furthermore, this research provides additional nomological validity checks of existing research constructs and their measurement items in the context of electronic recordkeeping systems and in the context of the culture of the New Zealand public sector.

\subsubsection{Practitioner Value of the Research}

Unfortunately, the level of acceptance and use of EDRMS has been mixed. With an increasing reliance on electronic records, the impact of low utilization of an organization's electronic recordkeeping system means that electronic documents are not being properly managed, can be lost through destruction or deterioration, or else they can be made irretrievable through a lack of a suitable indexing mechanism.

By identifying the factors that impact the use of electronic recordkeeping systems, this research helps identify and prioritize the factors that constitute the greatest barriers to use. Knowledge of these factors will help software developers to improve their system's user interface, provide trainers with critical insight from which they could develop training materials that target the most important knowledge gaps, and provide managers with the means to develop policies and change management strategies that will reduce barriers and ultimately increase the acceptance and use of these critical systems. 


\subsection{Thesis Outline}

The remainder of the thesis is structured in eight additional chapters as follows:

- Chapter 2: Literature Review

The literature review covers the importance of records and recordkeeping to society and introduces the primary models and theories supporting modern recordkeeping approaches and standards. The focus then moves to electronic recordkeeping systems, and finally to the introduction of constructs that are expected to influence the use of these systems.

- Chapter 3: Conceptual Research Model

In this chapter, the constructs introduced in the literature review are organized into a conceptual research model. The model consists of factors (constructs) that were selected to represent three conceptual areas: technology acceptance, organization context, and knowledge interpretation.

- Chapter 4: Research Design and Methodology

The research design and methodological approach is presented in this chapter. This includes consideration of the research paradigm and the definition of a suitable population from which to sample. The final research design, a mixed-methods approach consisting of a qualitative phase followed by a quantitative phase, is presented.

- Chapter 5: Phase I: Qualitative Methods

The qualitative data-gathering activities are discussed in detail in this chapter. These include three stages consisting of semi-structured interviews, proofreading of the measurement items, and the card sorting activities used to link the measurement items to the research model's constructs. Each stage consists of the methods, analysis, and findings, as each stage forms the foundation for the next stage.

- Chapter 6: Phase II: Quantitative Methods

This chapter focuses on the quantitative methods phase. This phase includes the design and development of the survey instrument, the survey pretest activities, and the final survey data-gathering activities and measurement approach. 
- Chapter 7: Phase II: Data Analysis

The data analysis chapter focuses on the data gathered during the final survey stage. This analysis includes descriptive statistics followed by factor analysis, which seeks to identify the most appropriate measurement items for inclusion in the final structural equation model. The statistical measurement of the model is covered, followed by the measure of the hypothesized path effects. The findings are further supported by a qualitative analysis of the open comments provided by the survey respondents.

- Chapter 8: Discussion

The discussion chapter brings all of the findings together. It starts by considering the measures of the constructs as they were evaluated in the qualitative and quantitative phases of the research. Next, the research model is discussed, including the implications of the empirical findings to the research hypotheses.

- Chapter 9: Conclusions

Finally, the conclusion chapter provides a summary of what the research has achieved in terms of answering the research questions, achieving the research objectives, and its contributions. The limitations of the research are discussed and several directions for possible future research are suggested.

\subsection{Chapter Summary}

In this chapter, the motivation for the research is introduced and the research gap is identified. The research question is then introduced, namely: What factors influence a user's intention to use an electronic recordkeeping system? This question lays the foundation for the two research objectives which seek to identify those factors, and once identified, determine the relative importance of the those factors.

The general research methodology is then described, as is the value of the research to both the academic and practitioner fields. The chapter concludes with a summary outline of the contents of the remaining thesis chapters. 


\section{Chapter 2}

\section{Literature Review}

Contents

2.1 Chapter Overview . . . . . . . . . . . 9 9

2.2 Records and Recordkeeping ......... 10

2.3 Recordkeeping Models and Theories . . . . . . 19

2.4 Electronic Document and Records Management 25

2.5 EDRMS-Use Constructs . . . . . . . . . 30

2.6 Chapter Summary . . . . . . . . . . 55

\subsection{Chapter Overview}

The literature review examines the foundation of electronic recordkeeping systems by first exploring the concept of a record and then providing an account of the evolving historical approaches to recordkeeping. Structuration Theory is introduced as the primary theoretical mechanism for considering a society's recordkeeping requirements, while the Records Continuum Model is introduced as the foundation for modern recordkeeping and archival science. The resultant electronic recordkeeping technology, the primary subject of this research, is introduced with a focus on its suitability to meet society's current and future recordkeeping requirements.

An account and consolidation of the most relevant Information Systems (IS) literature is then provided with consideration first given to the dependent variable: system use. The determinants of system use are then explored by focusing on factors associated with generic models for the acceptance and use 
of technology; on factors associated with the organizational context of the system, and finally on factors relating to the interpretation of the value of recorded knowledge by the system's users.

\section{$2.2 \quad$ Records and Recordkeeping}

Without reliable recorded evidence, we are, in fact, doomed to personal ignorance, cruel manipulation and exploitation, and ultimately societal chaos. (Cook, 1997, p. 23)

A record is defined in ISO 15489.1: Records Management ${ }^{1}$ as "information created, received, and maintained as evidence and information by an organization or person, in pursuance of legal obligations or in the transaction of business" (ISO 15489.1, 2002, p. 3). Records management is defined as the "field of management responsible for the efficient and systematic control of the creation, receipt, maintenance, use and disposition of records, including processes for capturing and maintaining evidence of and information about business activities and transactions in the form of records" (ISO 15489.1, 2002, p. 3). Records are maintained as evidence for the purpose of accountability (Oliver, Evans, Reed, \& Upward, 2009; ISO 15489.1, 2002; Cullen et al., 2000). They must be trustworthy in order to stand as evidence.

Many modern office workers spend their days creating documents. A document is defined as any "recorded information or object which can be treated as a unit" (ISO 15489.1, 2002, p. 3). With such a general definition, a document can appear in a wide range of forms (e.g., reports, letters, emails, memos, SMS text messages, scraps of paper, etc.). However, these information units are not records, or at least not until they have been placed under systematic control that can guarantee certain key characteristics required for use as evidence. As such, records and the process of records management are related and reliant on each other, for a record must be managed systematically in order to maintain the evidentiary chain.

Records management practices have developed over time to focus on managing and protecting a record's key evidentiary characteristics that contribute to the trustworthiness of that record. These characteristics are briefly discussed below.

\footnotetext{
${ }^{1}$ ISO 15489: Records Management is frequently referenced in this section. It is the international standard for records management and, by its nature, an influential and authoritative source.
} 


\subsubsection{Key Characteristics of Records}

Trustworthy records are essential for an organization to meet its legislative and internal business needs. From a records management perspective, the following characteristics are used to describe trustworthy records: authenticity, reliability, integrity, and usability (Sprehe, 2008; NARA, 2005; ISO 15489.1, 2002; Cullen et al., 2000; Upward, 1996).

\section{Authenticity (and Provenance)}

An authentic record is one that can be proven to be what it purports to be, to have been created or sent by the person purported to have created or sent it, and to have been created or sent at the purported time (ISO $15489.1,2002$, p. 7 ). To prove authenticity, records require policies and procedures that control and record the creation, transmission, receipt, and maintenance of records (NARA, 2005). These systematic controls ensure that records creators are authorized and identified and that records are protected against unauthorized addition, deletion, and alteration; in other words, a documented history of the record. This history of a record or object is called its provenance.

The term provenance is used extensively in the study of fine art, where a documented history helps an object to 'attain an authority'. It is a method of placing the object in a particular context and determining its importance relative to other objects (Moreau et al., 2008).

In the case of historic manuscripts, the need to determine provenance led to the creation of a body of knowledge within archival science known as diplomatics. Diplomatics is dedicated to examining clues that support provenance claims. One of the original definitions of diplomatics (circa $17^{\text {th }}$ century) was "the establishment of certain and accurate terms and rules by which authentic instruments can be distinguished from spurious, and certain and genuine ones from uncertain and suspect ones" (MacNeil, 2004, p. 202).

In modern times, the scientific, financial, and art communities find knowledge of provenance (for example, the ownership and/or access history) of objects to be of great importance as this history provides vital elements for determining the object's relative trust level (Hasan, Sion, \& Winslett, 2007). In the case of records, provenance has even been called the fundamental principle of archival science (Hasan et al., 2007) for its importance in contributing to a record's authenticity. 
The record of an object's provenance must itself also be a trusted record. Examples of domains that require secure provenance include law (where ownership history must be maintained if it is to be used as reliable evidence); authorship (where provenance records or evidence can be used to resolve timing disputes associated with research or patent applications); scientific data (tracking data and data transformation through large collaborative projects); digital forensics (any changes to data or documents would be known); and regulatory compliance ranging from trusted medical records to government and financial institution recordkeeping compliance legislation (Hasan et al., 2007).

\section{Reliability}

A reliable record is defined as one whose contents can:

be trusted as a full and accurate representation of the transactions, activities or facts to which they attest and can be depended upon in the course of subsequent transactions or activities. Records should be created at the time of the transaction or incident to which they relate, or soon afterwards, by individuals who have direct knowledge of the facts or by instruments routinely used within the business to conduct the transaction. (ISO 15489.1, 2002, p. 7)

From that definition, it can be noted that the context of the record's creation is important in determining its reliability. Automated, real-time capture of objective transaction information would have the highest reliability, whereas timely first-hand accounts by individuals would have slightly less reliability, as the content is necessarily filtered through personal subjective interpretation (Collopy, 1996). Again, a record's provenance or lineage can be used as evidence in support of a record's reliability (Simmhan, Plale, \& Gannon, 2005), with records created at the time of the event considered to be the most reliable.

\section{Integrity}

The integrity of a record refers to its being complete and unaltered. The ISO standard on Records Management states: 
It is necessary that a record be protected against unauthorized alteration. Records management policies and procedures should specify what additions or annotations may be made to a record after it is created, under what circumstances additions or annotations may be authorized, and who is authorized to make them. Any authorized annotation, addition or deletion to a record should be explicitly indicated and traceable. (ISO 15489.1, 2002, p. 7)

ISO 15489.1 emphasizes the need for systematic management policies and procedures to guard against alteration and fraud. This again illustrates the need to capture a complete and auditable record of a record's provenance as an important indicator of that record's integrity. The risk of fraud in the provenance record (in addition to fraud in the record itself), has led to the development of secure provenance, especially in electronic records (Hussein, Karim, \& Selamat, 2007). Furthermore, the systems themselves must have integrity. The perceived need for system integrity is also helping to build the case for the transparency commonly associated with open source software solutions (Ince, Hatton, \& Graham-Cumming, 2012).

Another aspect of integrity is the contextual and structural integrity of the content of records. This includes the physical and logical format and the relationships between content elements. Failure to maintain the structural integrity of records may impair a record's reliability and authenticity (NARA, 2005); but more importantly, it will impact the record's usability.

\section{Usability}

A usable record is one that can be found and understood in its original context. The ISO standard on Records Management further states:

A usable record is one that can be located, retrieved, presented and interpreted. It should be capable of subsequent presentation as directly connected to the business activity or transaction that produced it. The contextual linkages of records should carry the information needed for an understanding of the transactions that created and used them. It should be possible to identify a record within the context of broader business activities and functions. The links between records that document a sequence of activities should be maintained. (ISO 15489.1, 2002, p. 7) 
There are thus two aspects of a record's usability: the usability of the record itself and the functionality of the system that is managing the record. To keep an individual record usable, it must be preserved over time. In general, the primary goal of preservation is "to allow future users to retrieve, access, decipher, view, interpret, understand, and experience documents, data, and records in meaningful and valid (that is, authentic) ways" (Cullen et al., 2000, p. 54). One of the challenges for preservation is that records must be altered to preserve them, and this impacts on the record's integrity and authenticity (Cullen et al., 2000). Clearly, a balance must be sought to maintain authenticity and usability.

The second aspect of usability relates to the functionality of the system in which the record is being managed. The definition requires that a usable record be able to be "located, retrieved, presented and interpreted." In order to do that, sufficient information must be captured about the record and the context of its creation and use. This information should be sufficient to locate the record, while the contextual linkages should provide an understanding of the transactions that created and used them (NARA, 2005).

\subsubsection{A Short History of Records and Recordkeeping}

The history of records and records management in western civilization is presented in this section. ${ }^{2}$ The three models of records management covered below have been refined over time to meet the evidence requirements of the day, and now form the foundation for much of our current legal system and economy.

\section{The Archive}

Western civilization's approach to recordkeeping can be traced back thousands of years to ancient Greece. The ancient Greeks considered trustworthy records to be the key to a trustworthy government. In fact, the word 'archive' (Greek: archeion) describes the 'keystone' of an arch, thereby illustrating the role that records play in preserving the delicate 'arch of government'. To safeguard these critically important records, the ancient Greeks created a secure repository location in which they could store their historical recorded evidence.

The Romans expanded the scope of their archivum by providing a public office in which citizens could record their transactions, thereby providing a

\footnotetext{
${ }^{2}$ Much of the historical context featured in this section is sourced from Upward's seminal work found in Archives: Recordkeeping in Society (Upward, 2005).
} 
location and legitimizing mechanism to create and guard the authenticity and reliability of their records. The archive became the safe house as well as the formal witness of action. Much of the success of Rome and its wealth from trade can be attributed to the confidence that merchants had in the 'truth' of the records lodged in the archivum (Duranti, 1996; Upward, 2005).

This tradition has continued, with most modern nation states continuing to support national archives, an institution (and location) to guard the authenticity and reliability of their national records and history (Duranti, 1996).

\section{Archival Diplomatics}

The period following the fall of the Roman empire coincided with the loss of the Roman archivum as witness and guardian of the community's official records; however, the need for authenticity and reliability of documents (especially representing transactions or treaties) continued as a necessary part of a functioning marketplace or government. Lacking an archivum as the location/witness to provide legitimacy to documents, a mark of authenticity and reliability was instead embedded into the document itself. The professions of the notary and the jurist came into being. This meant that an official state-appointed person provided that mark of legitimacy on the document (according to the rules of diplomatics) as a method of determining authenticity and the provenance of these official documents in order to guard against fraud (MacNeil, 2004).

In other words, the methods of managing the key characteristics of a record (authenticity, reliability, integrity, and usability) effectively split into two camps. On the one hand, there was the archive, a secure storage location and official witness of transactions. On the other hand, there was the autonomous record (e.g., diplomas, certificates, etc.) signaling its trustworthiness through embedded markers of authenticity and reliability, supported by a range of officials trained to make and read those embedded markers.

\section{Bureaucratic Recordkeeping}

The third wave of change came about during the rise of nation states, global colonization, and 'big business', resulting in the development of the bureaucratic recordkeeping approach (Upward, 2005). Organizations were beginning to rival governments in complexity and required standardized records to run geographically dispersed operations. They duly created powerful systematic 
control systems called registries. These registries (which were often located in regional offices, in contrast to the archive's single, central location) effectively managed metadata (i.e., data about data) for two primary purposes: describing the records themselves, and describing the relationships between records. Registrars (the keeper of the registries) were now the guardians of authenticity, reliability and integrity for the new systems.

The bureaucratic recordkeeping systems supported the current operations of organizations, and their records often had a relatively short period of value. Modern techniques and theory developed during this time focused on managing a record's life-cycle, and these helped to guide records managers on rules of creation, use, storage, and eventual disposal of records (Upward, 2005).

Today, western civilization relies on all three methods to maintain reliable recorded evidence. ${ }^{3}$ Nation states actively maintain national archives that store and maintain records of events and activities. Official documents are still issued with embedded markers of authenticity or an official witnessing by a notary or justice of the peace, and organizations and governments are still operating official recording departments to maintain registries of transactions and operational activities. Nonetheless, the point at which each key characteristic is sufficient to raise the status of a document to that of a record is contingent on the interpretation of that characteristic and on the needs as defined by the legislative and regulatory setting of the society in which the record resides.

\subsubsection{A Short History of Electronic Recordkeeping}

During the 1970s, along with the introduction of computing and computerized information systems, the recordkeeping and archival community found itself at a crossroads. Information was increasingly being stored electronically, and these electronic records didn't fit well in their paper-based, records-life-cyclemodeled world. One side argued that archival work was scholarly and that the methods supporting historical research should be shifted from a record's content to its context as a way of dealing with the volume and format of these new electronic records (Cook, 1997, p. 19). The other side proposed that archivists should "abandon their traditional history-based orientation, and

\footnotetext{
${ }^{3}$ Although all three methods are in use in modern western civilization, the EDRMS shows the legacy of the Roman archivum - a central repository to guard the authenticity and reliability of its records.
} 
transform themselves into records or information managers, into computer scientists, into technical experts in the new media" (Cook, 1997, p. 19).

In addition, separating the digital information streams and declaring which one is the record and which one is not is more difficult than it would appear. For example, a digital office document may be considered self-contained, whereas a database query is only transitory. Speaking of David Bearman's contribution to the recordkeeping literature, Cook noted that electronic files, such as computer dumps or backups, are not evidence of business transactions, but rather "views or frozen snapshots of information, and views that are by definition incomplete, not linked to creator context or activity, and therefore without assurance of reliability and authenticity. They are not records" (Cook, 1997, p. 23).

At what point does an electronic file become worthy of being called a record? What are the attributes of an electronic record and how can those attributes be applied in the electronic medium where physical form is meaningless?

Before an electronic recordkeeping system could be introduced into practice, the definitions and attributes of an electronic record had to be determined. Starting in the 1990s, there have been several efforts to develop these definitions and to set standards for electronic records and electronic recordkeeping, including: the Pittsburgh Project (Pittsburgh Project, 1993); the ongoing InterPARES project (Gilliland, Rouche, Lindberg, \& Evans, 2005; Duranti \& Blanchette, 2004; MacNeil, 2002; InterPARES Project, 2011); and the Clever Recordkeeping Metadata Project (Evans, Reed, \& McKemmish, 2008; Final Report CRKM Project, 2007; Evans, McKemmish, \& Bhoday, 2006; CRKM Project, 2011). The outcome of these projects was the creation and standardization of recordkeeping metadata.

Recordkeeping metadata is defined as including "all standardised information that identifies, authenticates, describes, manages and makes accessible, through time and space, documents created in the context of social and business activity" (McKemmish, Acland, Ward, \& Reed, 1999, p. 7). Additional work in refining models of recordkeeping metadata (McKemmish et al., 1999; Evans \& Lindberg, 2004; Final Report CRKM Project, 2007) has resulted in a refined conceptualization of recordkeeping metadata that became an international standard, ISO 23081.1 (2006) - Managing metadata for records. An example of an electronic recordkeeping metadata model for use in a business and socio-legal context is provided in Figure 2.1.

In the next section, the discussion will focus on the theoretical underpinnings of how records and society relate to, and support, each other. 


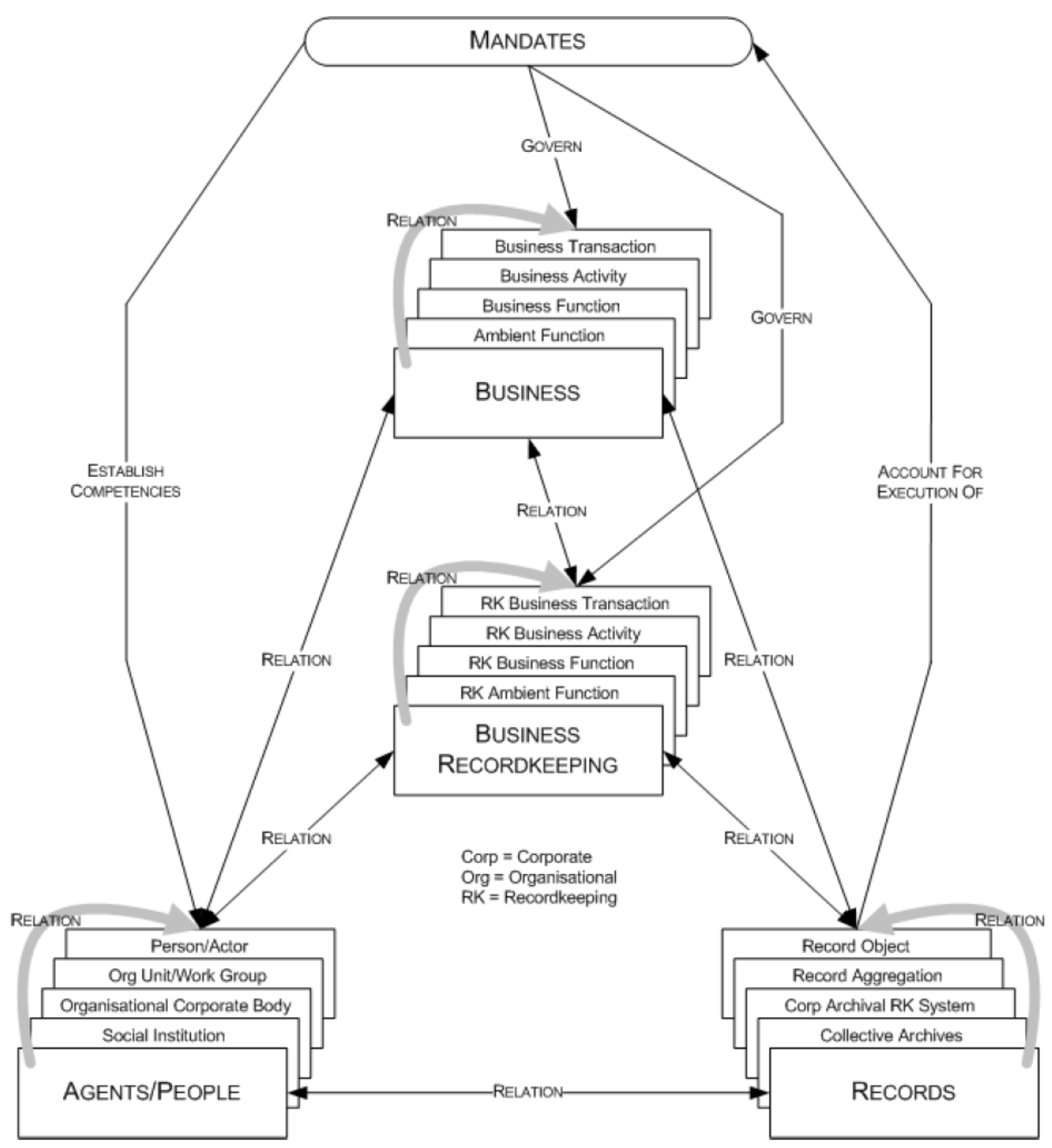

Figure 2.1: Conceptual Model of Recordkeeping Metadata in Business and Socio-Legal Contexts (Final Report CRKM Project, 2007, p. 4) 


\subsection{Recordkeeping Models and Theories}

Recordkeeping has evolved primarily out of practice rather than theory. As society has increased its requirements for maintaining reliable evidence, recordkeeping has sought to meet those requirements through evolving mechanisms, with the aim of maintaining reliable and trustworthy records to a level that is acceptable in that society. As such, recordkeeping is primarily governed by the norms and needs of the society that it supports. However, societies change over time, and given the complexity and volume of information created in today's digital age, it has been necessary to develop a theoretical foundation to build new solutions and information strategies. Giddens' structurational concept of space-time distanciation is the primary theoretical concept underpinning Upward's development of the Records Continuum Model (Upward, 1997). These are both discussed below.

\subsubsection{Structuration Theory}

Structuration Theory is a general theory of social organization (Giddens, 1984). It is a commonly accepted platform upon which we view and consider people's actions and the structure of society as they recursively evolve over time (M. Jones, Orlikowski, \& Munir, 2004; M. Jones \& Karsten, 2008). It consists of a model to explain the relationships between individuals (agents) and the structure of the society in which they reside (structure). At its heart, it attempts to reconcile the apparent theoretical dichotomies of social systems such as agent/structure, micro/macro, subjective/objective, etc. As it is dealing at a theoretical level (a theory of theories), Weaver and Gioia go so far as to state that "Structuration is a bona fide meta-theory" (Weaver \& Gioia, 1994, p. 579).

At the time of Giddens' writings, there were two competing traditions in sociology. There was the tradition of naturalistic sociology (positivism) that argued that there were relatively strong social laws and external social structures that acted on relatively passive individuals as a way of explaining social phenomena (Proctor, 2005). There was also a competing interpretive tradition that saw the actions of individuals (through phenomenology and ethnomethodology) as producing the phenomena of larger social structures (M. Jones et al., 2004, p. 300).

Giddens found this dualism to be unsatisfactory, and proposed that structure and human agency should be perceived as a mutually constitutive duality. In other words, he found a middle way between positivism and 
interpretivism where societal structure is the outcome of human agency and practices, but it is also the medium in which those practices take place and is thus influencing both the shape and form of those practices. In this light, societal structure is both affecting individuals and being affected by those individuals simultaneously. Giddens' use of the term structuration invokes the condition of societal structures as being in a constant state of creation through the flow of everyday social practices (M. Jones \& Karsten, 2008, p. 131).

To assist with analysis, Giddens created a model with three dimensions of structure (signification, dominance, and legitimation) that interact with three dimensions of interaction (communication, power, and sanction) through what he terms modalities (interpretive schemes, facility, and norms). See Figure 2.2 below.

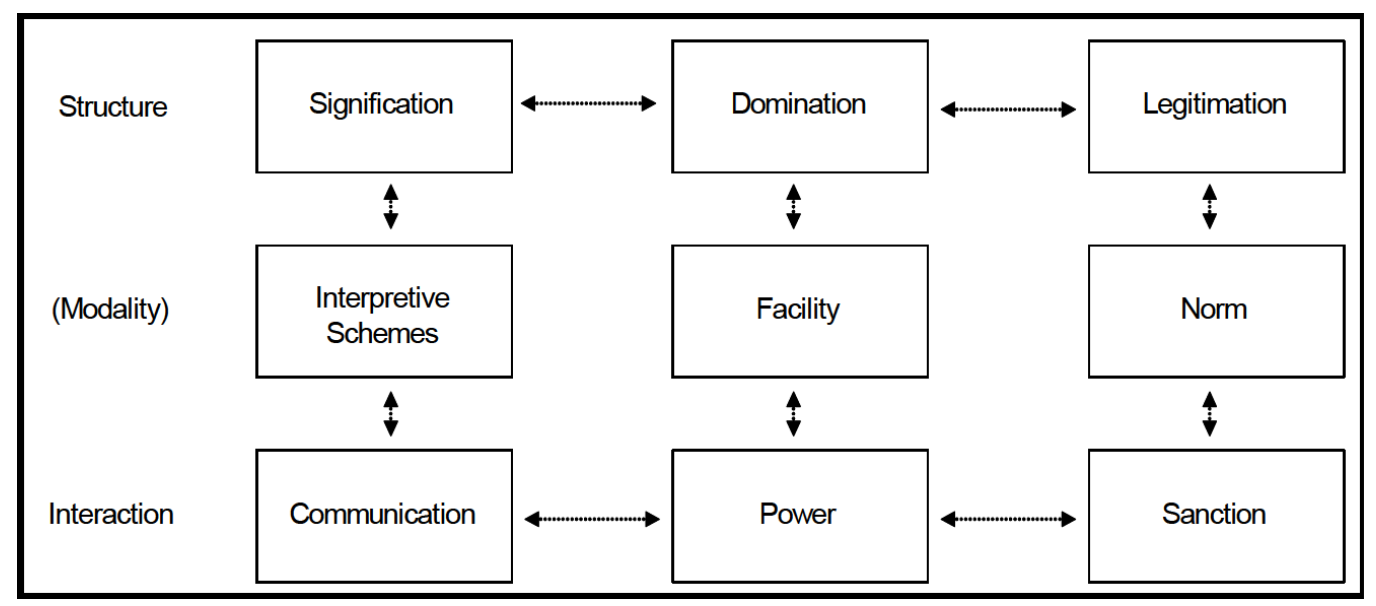

Figure 2.2: The Dimensions of the Duality of Structure (adapted from Giddens, 1984 , p. 24)

Jones and Karsten used clothing to illustrate Giddens' model of the duality of structure (M. Jones \& Karsten, 2008, pp. 129-130). The selection and form of clothing is a structure of the society. Doctors wear white coats to communicate a signification of their role in the societal structure. People in hospitals understand and expect these white coats and are able to interpret their significance. As an example of domination, the police and military utilize uniforms with insignia to communicate power and dominance roles to other members of their societies. Legitimation is less about communicating significance or dominance and more about meeting expectations for normal 
and acceptable behavior; for example, in the degree of formality of dress allowed in an office situation (e.g., casual Fridays).

The concept of space-time distanciation is a recurring theme in Giddens' work, and refers to the intersecting points of space and time as being necessary coordinates to determine the structure of society at that place and that time. Giddens sees structuration as involving three 'intersecting planes of temporality': durée (the timeframe of day-to-day life), dasein (the human lifespan), and the longue durée (the timeframe of social institutions), thus illustrating that his theory is able to link the individual to that of the longerlived social institution (M. Jones \& Karsten, 2008, p. 133).

However, Giddens' structuration theory is often considered too broad to be used by itself. Poole \& DeSanctis note that:

[S]tructuration theory has been the theoretical lens of choice for most scholars during the past decade. It provides a major theoretical pillar, though it specifies no detailed theorems or formal hypotheses; and it offers little methodological guidance. [...] [S]tructuration is more a guiding philosophy of social scientific inquiry than a theory per se. It offers a grand formulation - a way of viewing the world - that is so general and encompassing that it cannot be falsified. (Poole \& DeSanctis, 2004, p. 207)

Nevertheless, Giddens' theoretical approach to explaining the constitution of society does provide a logical foundation for understanding how societies form and change their norms over time. This movement at the societal level also informs the changing level of acceptability that defines a record over time - an important attribute in defining recordkeeping requirements. Upward (1997) explored this implication to recordkeeping while developing his Records Continuum model.

\subsubsection{The Records Continuum Model}

For the greater part of the $20^{\text {th }}$ century (up through the $1980 \mathrm{~s}$ ), the dominant model controlling the records management-archives relationship consisted of the life-cycle model (J. Atherton, 1985; Gilliland et al., 2005; Johnston \& Bowen, 2005).

The life-cycle model is based on the premise that the life of a record can be divided into eight separate stages in two phases, starting with a records management phase consisting of the 
1. creation or receipt of information in the form of records,

2. classification of the records or their information in some logical system,

3. maintenance and use of the records, and

4. their final disposition through destruction or transfer to an archive.

This is then followed by an archival phase consisting of the

5. selection/acquisition of the records by an archive,

6. description of the records in inventories, indexing, etc.,

7. preservation of the records or, perhaps, the information in the records, and

8. reference and use of the information by researchers and scholars ( $\mathrm{J}$. Atherton, 1985).

The two phases were most often managed by completely separate organizations, with the records management phase being managed by the organization based on its needs, followed by a handover of the records to an archival organization supporting a different set of users and stakeholders governed by different policies and procedures. Inevitably, the archivist had little or no input into how records were initially captured, and the records manager often neglected to identify permanently valuable records for the future.

In 1985, Atherton stated that the "split between the records management and archival phases of the life-cycle is no longer acceptable" and recommended merging the recordkeeping and archival phases into a single continuum to help maintain contextual continuity throughout the managed life of a record (J. Atherton, 1985, p. 47). At the same time, archives were under a growing 'threat' by the spread of electronic documents and the Internet. In 1996, Upward noted that "[i]f the archival profession is to avoid a fracture along the lines of paper and electronic media, it has to be able to develop ways of expressing its ideas in models of relevance to all ages of recordkeeping" (Upward, 1996, p. 269).

To help practitioners move to a more unified approach to the recordsarchival relationship, Upward introduced a new model based on Giddens' notion of time-space distanciation (Giddens, 1984). Upward's model introduced a space-time model for records. In short, a record can be and be 
interpreted in many dimensions across time. For example, they can be created (as a record) and created again (either in new contextual groupings or in reinterpretation) without the need for a linear approach to time.

Upward described the new Records Continuum model as:

a major transition in archival practice. That transition involves leaving a long tradition in which continuity was a matter of sequential control. Electronic recordkeeping processes need to incorporate continuity into the essence of recordkeeping systems and into the life-span of documents within those systems. (Upward, 1997, p. 11)

Upward's Records Continuum Model created an intersection of the main records life-cycle processes (creation, capture, organization, and pluralization) and dimensions of 'recordness' including evidentiality, transactionality, recordkeeping containers, and identity. These were expressed metaphorically in concentric circles, where each of the processes could take place at intersections of different user dimensions in a sort of records space-time. See Figure 2.3.

The concentric circles expand into different user dimensions, which consist of different stakeholders and their requirements. These relationships are not necessarily linear, but can react and interact across both time and space. For example, the record itself is, by definition, a snapshot in time; however, how the record is interpreted and re-presented can change depending on the view and the context of its use.

The four dimensions include:

1. Dimension 1 Create: This dimension focuses on the primary actors, the acts themselves, and the documents that record the acts and trace and represent the acts.

2. Dimension 2 Capture: This dimension focuses on the personal and organizational recordkeeping systems and the policies that ensure that records are captured in context and in sufficient detail for use as evidence.

3. Dimension 3 Organize: The third dimension focuses on the recordkeeping processes within an organization. It ensures that the recordkeeping regime is sufficient to form an accurate memory of its business and social functions.

4. Dimension 4 Pluralize: The forth dimension embeds the records into the society in which they reside. It ensures that the records can be 


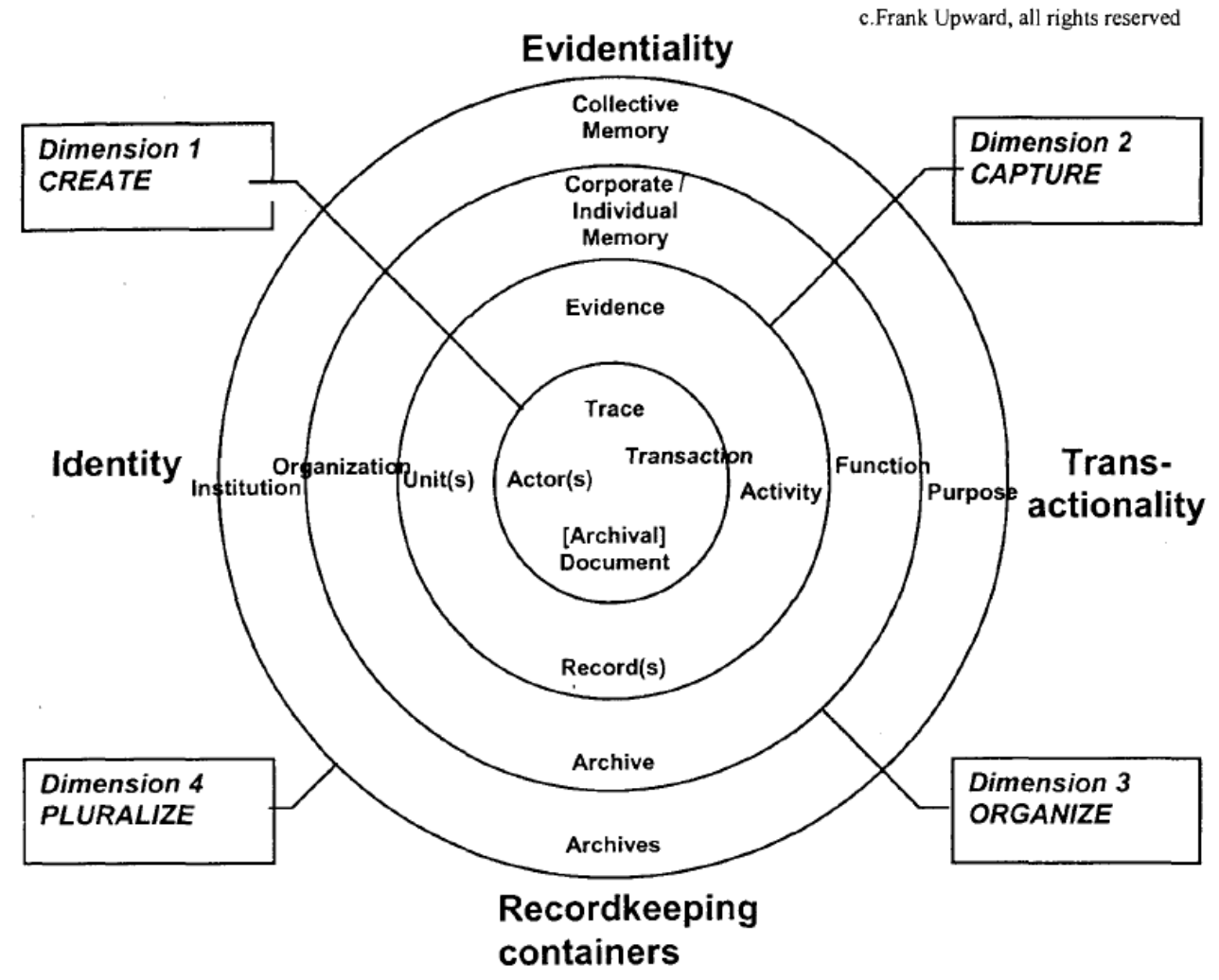

Figure 2.3: The Records Continuum - a spacetime model (Upward, 2005)

carried beyond the life of an organization or individual and add to the collective social, historical, or cultural memory (McKemmish, 1997; Upward, 1996, 1997).

McKemmish summarized the records continuum model as a way of thinking about recordkeeping in both our organizations and in our society (McKemmish, 1997; Upward, 2005). In this light, the model helps to identify key evidential, recordkeeping, and contextual features of the continuum and places them in relationship to each other. It also represents the multidimensional nature of the recordkeeping function. It maps the evidential, recordkeeping, and contextual features of the continuum against the dimensions of the recordkeeping function, and it finally places it in a broader socio-legal and technological environment (McKemmish, 1997).

In 2000, with the assistance of Reed and Schauder, Upward introduced additional continuum models, including the Information Continuum Model, 
the Information Systems Continuum Model, and the Publishing Continuum Model (Upward, 2000). All of the models use the same dimensions but with different processes, and represent the "intra-disciplinary information management and systems nature of records management, as it does for each of the specialisations depicted" (Upward, 2000, p. 133).

The continuum approach to records as they exist in both space and time is critical to the mental move required to address the functional requirements and need for virtual archives to manage the future's electronic records (Cook, 1994). Nevertheless, both structuration theory and the related records continuum model are an abstraction of reality - and they still require a tangible method of application to recordkeeping in general and electronic recordkeeping specifically.

\subsection{EDRMS: Electronic Document and Records Management Systems}

By the turn of the millennium, the current recordkeeping theory, in the form of Upward's Records Continuum Model (Upward, 1996, 1997, 2000), had caught the attention of much of the recordkeeping profession and had formed the basis of the international standard ISO 15489: Records Management. Furthermore, work had begun on defining the model of electronic recordkeeping metadata (later published as ISO 23081: Managing metadata for records - see example on p. 18) that would be required to make electronic recordkeeping technically possible. The focus now turned to developing a system to create and manage electronic records.

\subsubsection{Reorganizing Recordkeeping}

In 2001, the State Records Authority of New South Wales (Australia) created a strategy called DIRKS: Designing and Implementing Recordkeeping Systems (National Archives of Australia, 2001). This strategy was designed to assist organizations in setting up a compliant enterprise-wide recordkeeping system.

More recently, standard-setting authorities have built upon this foundation and either developed or adopted improved standards. Examples include the International Council on Archives (ICA)'s "Digital Recordkeeping Standard" (2010), as well as checklist-style functional requirements on electronic recordkeeping like those developed under the Australasian Digital Record- 
keeping Initiative and adopted by the ICA, such as "Principles and Functional Requirements for Records in Electronic Office Environments" (2008).

DIRKS and other national and international manuals have provided a practical method for an organization to determine and document their recordkeeping requirements and develop their contextual metadata strategies. For an example of recordkeeping metadata in use, see Figure 2.4.

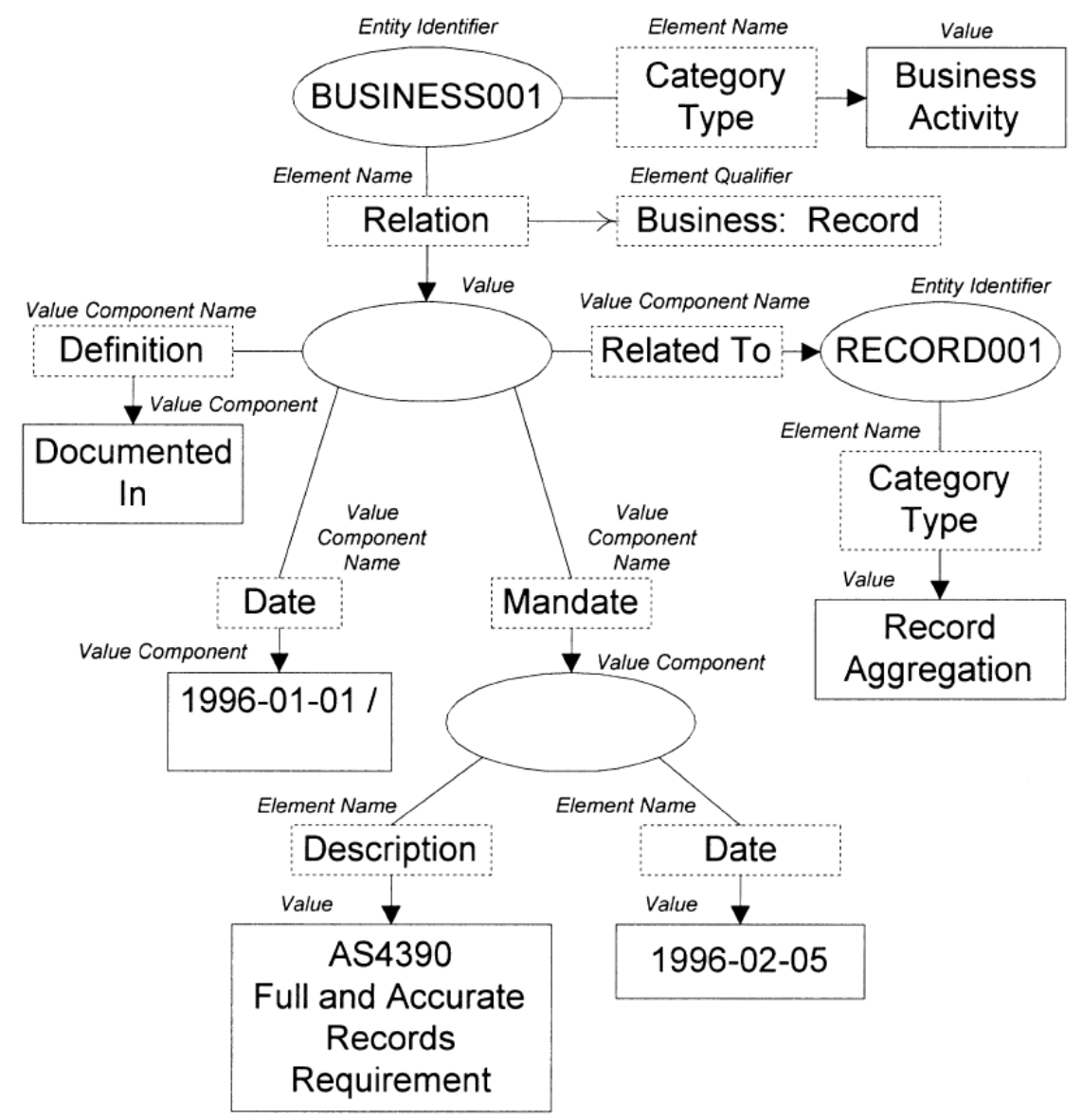

Figure 2.4: The recordkeeping metadata relationship between a record of a loan application (RECORD001) and the loan application business activity (Business001) (McKemmish, Acland, Ward, \& Reed, 1999, p. 25)

The creation of recordkeeping functional requirements created a suitable foundation for a technology solution that could be used in digital office environments. This technology gap was rapidly filled in the market by a number of commercial electronic recordkeeping systems. 


\subsubsection{EDRMS Defined}

When focusing on possible enterprise-wide solutions, Bearman made a strong differentiation between information systems and recordkeeping systems (Cook, 1997, p. 23). The former cover the storage and retrieval of both information (as in data fragments in a database) and documents, while the latter focus on the more formal requirements of records management, which include protecting the key characteristics of records such as authenticity and integrity (see Section 2.2.1).

In the traditional life-cycle model, documents were generally only embedded in a formal recordkeeping system (archive) after they had satisfied their initial purpose within the organization (often years after). However, the records continuum model introduced a 'spacetime shift' in the perception of records (Upward, 2000, p. 126). The result of this shift in thinking was ultimately to push the ideal requirement for formal recordkeeping as close to the moment of creation as possible.

It logically follows that any electronic systems that were designed to manage records should also be intimately involved with their creation. In an effort to reconcile Bearman's differentiation between information systems and recordkeeping systems in terms of the management of documents, Benfell (2002) stated that a single, combined Electronic Document and Records Management Systems (EDRMS) would generally be the most effective enterprise-wide solution. Several EDRMS solutions soon entered the marketplace to focus on the management of 'office'-type documents.

The State Records of South Australia (SRSA), for example, defines an EDRMS as:

An automated system used to manage the creation, use, management and disposal of physical and electronically created documents and records for the purpose of supporting the creating, revision and management of digital documents, improving an organisation's work-flow and providing evidence of business activities. (SRSA as cited by Nguyen, Swatman, \& Fraunholz, 2008b, p. 524)

Efforts to create standards for electronic recordkeeping systems software soon followed, with some early examples including the the European Commission's MoReq: Model Requirements for Electronic Records (Cain, 2002) and the United States Department of Defense's Electronic Records Management Software Applications Design Criteria Standard (DoD 5015.02-STD, 2007). 
The electronic recordkeeping software standards continue to develop. With the release of MoReq2010 (Lappin, 2011a; MoReq2010, 2011), which includes a new modular requirements structure, standard XML schema, and a vendor certification process, the commercial offerings in the electronic records management software space could expand significantly (Cunningham, 2011). In spite of some process concerns (Fresko, 2010), it is likely that electronic recordkeeping standards and resultant technology solutions will continue to change rapidly to meet the evolving needs of the organizations and societies that they support.

\subsubsection{The Future of EDRMS}

The development of national and international recordkeeping standards and the push to develop electronic recordkeeping systems arrived during the collapse of the dot-com bubble and after a number of high profile accounting scandals including the collapse of WorldCom (Ackman, 2002) and Enron (Holtzman, Venuti, \& Fonfeder, 2003). A 2007 review of recordkeeping legislation in force for Australia, the USA, the UK, and the EU reveals that "records management has gradually moved from a position on the very periphery of organizational attention towards a far more central legal requirement for both public and private sector organizations" (Nguyen \& Swatman, 2007). Given the legislative imperative, the operational benefits, and the rapid adoption by large organizations, the EDRMS quickly joined other wholeof-enterprise systems, which include Enterprise Resource Planning (ERP), Customer Relationship Management (CRM), and Supply Chain Management (SCM) (Nguyen et al., 2008b). EDRMS as a class is now the predominant solution available to organizations to centrally manage their electronic records.

Harries (2009) successfully predicted that the EDRMS would be a foundational technology that would support what he terms a true "business transformation". Harries argued that EDRMS are the key enabler for two main reasons: 1) "e-records are one aspect of the modernization process, because the electronic management of electronic information is a necessary enabler for sustainable business change", and 2) "that better management is essential to meet information policy and compliance requirements in this modernised environment" (Harries, 2009, p. 16). He concluded that organizational management and public policy is undergoing a radical shift, and argues that larger, networked recordkeeping systems are required in order to move the perception of electronic records management from "a 'back-office' 
end-of-process activity to one that is central to the delivery of outcomes" (Harries, 2009, p. 24).

With the introduction of Microsoft Sharepoint, which focuses more on collaboration than recordkeeping (Lappin, 2011b), and the continued fragmentation of electronic document repositories, many began to think that EDRMS might be a passing fad. Lappin (2011a) thought that declaring the death of EDRMS was premature: "EDRMS has lost momentum but most of those organisations that have succeeded in implementing them corporate-wide have felt a benefit and will continue to maintain and develop their systems." It is likely that as the information systems ecology continues to evolve, the EDRMS will continue to evolve with them, taking the lead role in some cases and working in tandem or even behind the scenes with others.

Nonetheless, EDRMS are not without their limitations. In terms of recordkeeping models, the EDRMS is based on the records repository model (Lappin, 2010). The current electronic file standards do not (yet) include sufficient and trustworthy information to make individual files into records in their own right; so it remains the act of lodging the electronic file into an EDRMS that provides it with its legitimacy. Furthermore, the process of lodging the document remains a manual one (Joseph et al., 2012; Bailey, 2009).

Another limitation of EDRMS applications is that they typically only manage the 'office'-type documents, and often exclude records represented in other enterprise business systems and Web 2.0 platforms (Lips, Rapson, \& Hooper, 2008; Lips \& Rapson, 2009, 2010), which arguably create the bulk of 'records' in most organizations. Reed (2008) advocated a significant scope enhancement to the EDRMS concept that would expand its domain to include automated collection of evidence of transactions taking place in other systems across the enterprise via the use of a common service-oriented architecture. $^{4}$

This concept of comprehensive or ubiquitous recordkeeping has broader implications for the structure of future organizations and for the discipline of records management. A current direction of research seeks to explore the broader impact (and opportunity) of weaving records management principles throughout an organization under the banner of recordkeeping informatics (Oliver et al., 2009, 2010; Upward, Reed, Oliver, \& Evans, 2013). In addition, there were also efforts to embed recordkeeping principles directly into the

\footnotetext{
${ }^{4}$ This functional requirement is supported by the MoReq2010 standard (Lappin, 2011a; MoReq2010, 2011), with technical standards modules being released from late 2012.
} 
'office'-type file formats (Mishina, Yoshihama, \& Kudo, 2007), which could introduce a new 'diplomatics' aspect to the measure of reliability in electronic records stored outside of an EDRMS repository. Furthermore, advances in cloud computing seek to redefine the concept of the 'records repository' while potentially increasing the portability (and potential of ubiquity) of records to anywhere on the Internet (Duranti, 2012; Lappin, 2010).

\subsection{Constructs Associated with the Use of EDRMS Technology}

The previous sections explored the literature with a focus on records, recordkeeping, and the purpose and importance of an EDRMS in modern organizations. Returning to the research question, the focus will now turn to the acceptance and use of EDRMS in the organizational context. The dependent variable, system use, is considered in Section 2.5.1, followed by an exploration of relevant factors that could potentially influence EDRMS-use in Section 2.5.2.

\subsubsection{System Use: the Dependent Variable}

Information systems are generally implemented in organizations with the expectation that they will be used by staff to the benefit of the organization. It is not surprising that the system use construct appears widely in the literature and has been under continual investigation since the 1970s (Barkin Gary \& Stephen, 1977, as referenced by Burton-Jones \& Straub, 2006). IS research has since dedicated much study and effort to understanding and measuring many aspects of the acceptance and use of technology (DeLone \& McLean, 1992; Venkatesh et al., 2003), using both variance and/or process research models (Fidock \& Carroll, 2009). See Figure 2.5.

Both approaches provide a partial understanding of the system under study. Variance research largely utilizes quantitative, survey-based methods, where measures are carefully developed to ensure their statistical validity and reliability. In variance research, researchers seek to identify which independent variables best predict the variation in occurrence or the magnitude of the dependent variable under study (Burton-Jones \& Straub, 2006). However, given the reliance on survey-based methods, variance models are also limited in scope to being 'snapshots' in time, where the selection of the time-slice is dependent upon the requirements of the research at hand. 


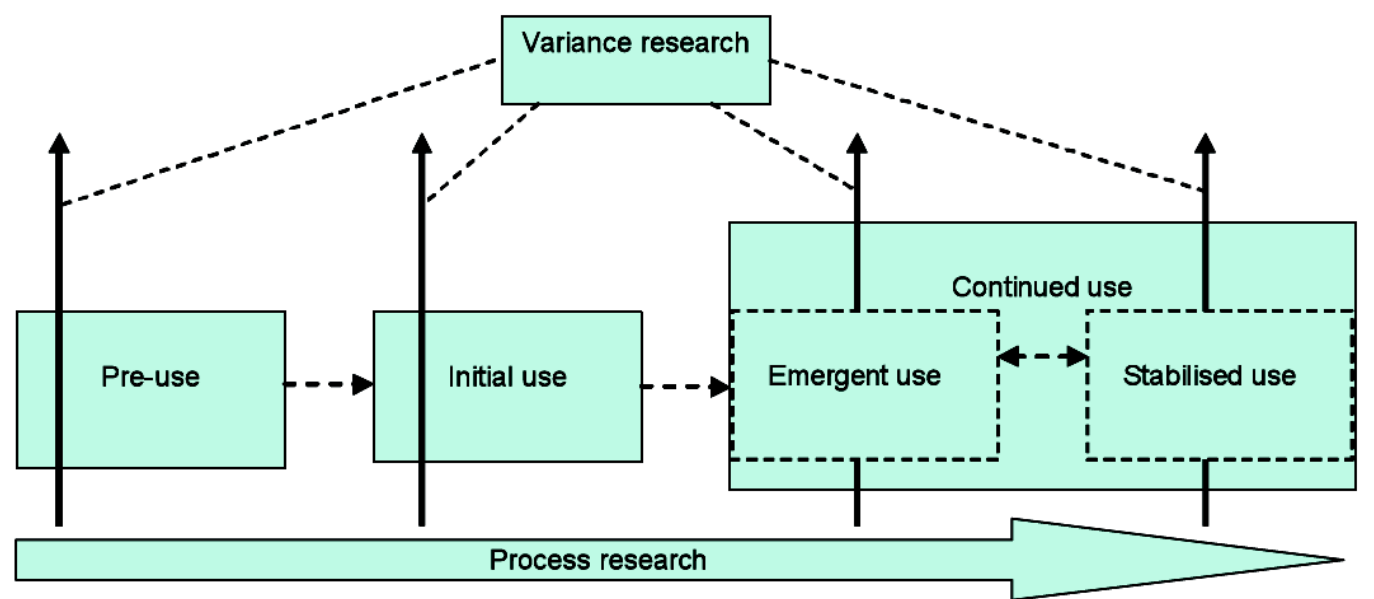

Figure 2.5: Representing and exploring system use from both variance and process perspectives (Fidock \& Carroll, 2009, p. 238)

In contrast, process research typically focuses on qualitative descriptions and focuses analysis on the unfolding nature of the phenomena of interest. Qualitative research methods are preferred for process models as they provide richer descriptions of the nature and sequence of events over time (Fidock \& Carroll, 2009).

IS research has approached the system use construct from many different angles. One example, the IS Success literature, focused on large metamodels that connected system use to organizational net benefits (DeLone \& McLean, 1992; Seddon, 1997; DeLone \& McLean, 2003). DeLone and McLean introduced their initial IS Success Model in 1992, which linked use and user satisfaction to individual impact as a predictor/mediator of organizational impact (DeLone \& McLean, 1992). See Figure 2.6.

Seddon critiqued the DeLone \& McLean IS Success Model as having "both a variance and an event-in-a-process interpretation" (Seddon, 1997, p. 242), and that such "muddled thinking" would be counterproductive for future IS research.

Seddon introduced an alternative to the DeLone \& McLean IS Success Model model in his 1997 respecification. Seddon's interest in transforming DeLone \& McLean's IS Success model into a variance model was primarily to facilitate the use of quantitative research techniques. This included the introduction of four new variables: expectations, consequences, perceived 


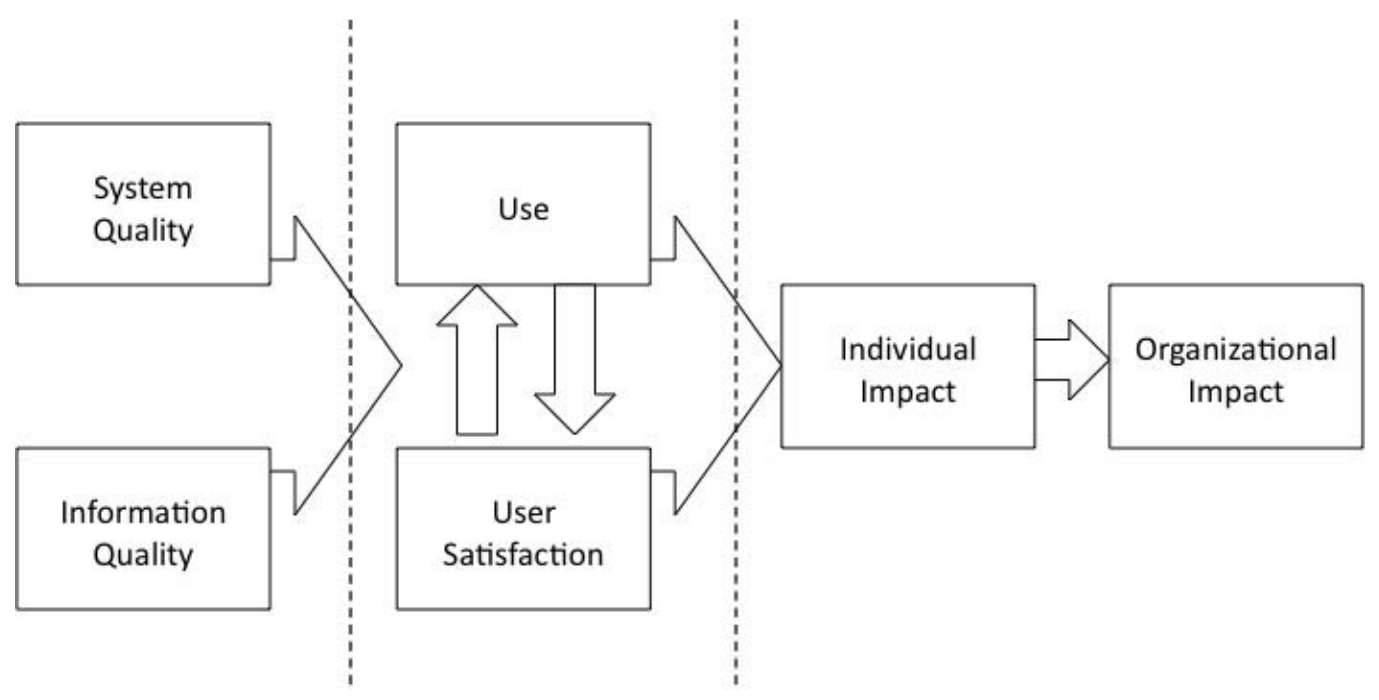

Figure 2.6: DeLone and McLean IS Success Model (DeLone \& McLean, 1992)

usefulness, and net benefits. See Figure 2.7 (p. 33). In this way, success became reliant on use.

Seddon further argued that system use is a behavior, not a success measure (Seddon, 1997, p. 244). He suggested that measures of net benefits would better serve as an independent input to a user's perceived usefulness and user satisfaction, and further suggested that net benefits could be measured in terms of individuals, organizations, and/or to society. ${ }^{5}$

Applying Giddens' Structuration Theory (see Section 2.3.1), the use of information technology initially creates the perceived benefits of that use, which in turn affects and drives the user's perception and likelihood of further use of that technology, thus demonstrating the complex relationship as they recursively evolve over time. Given the potential multidirectional relationship of system use with other constructs, it is unsurprising to find that it has been treated as both the dependent and independent variable in various research models. See Figure 2.8 (p. 34).

A particularly relevant definition of system use is: "an individual user's employment of one or more features of a system to perform a task" (Burton-

\footnotetext{
${ }^{5}$ Note: another stream of IS research has focused on how to measure the benefits of information system use on organizational performance (Melville, Kraemer, \& Gurbaxani, 2004). This line of research generally focuses on the 'bottom line', and uses profitability as the final arbiter of IS success. However, the relationship of trustworthy electronic records to profitability - particularly in the Public Sector, which is largely adopting EDRMS technologies for ethical and legislative reasons - would be tenuous at best.
} 


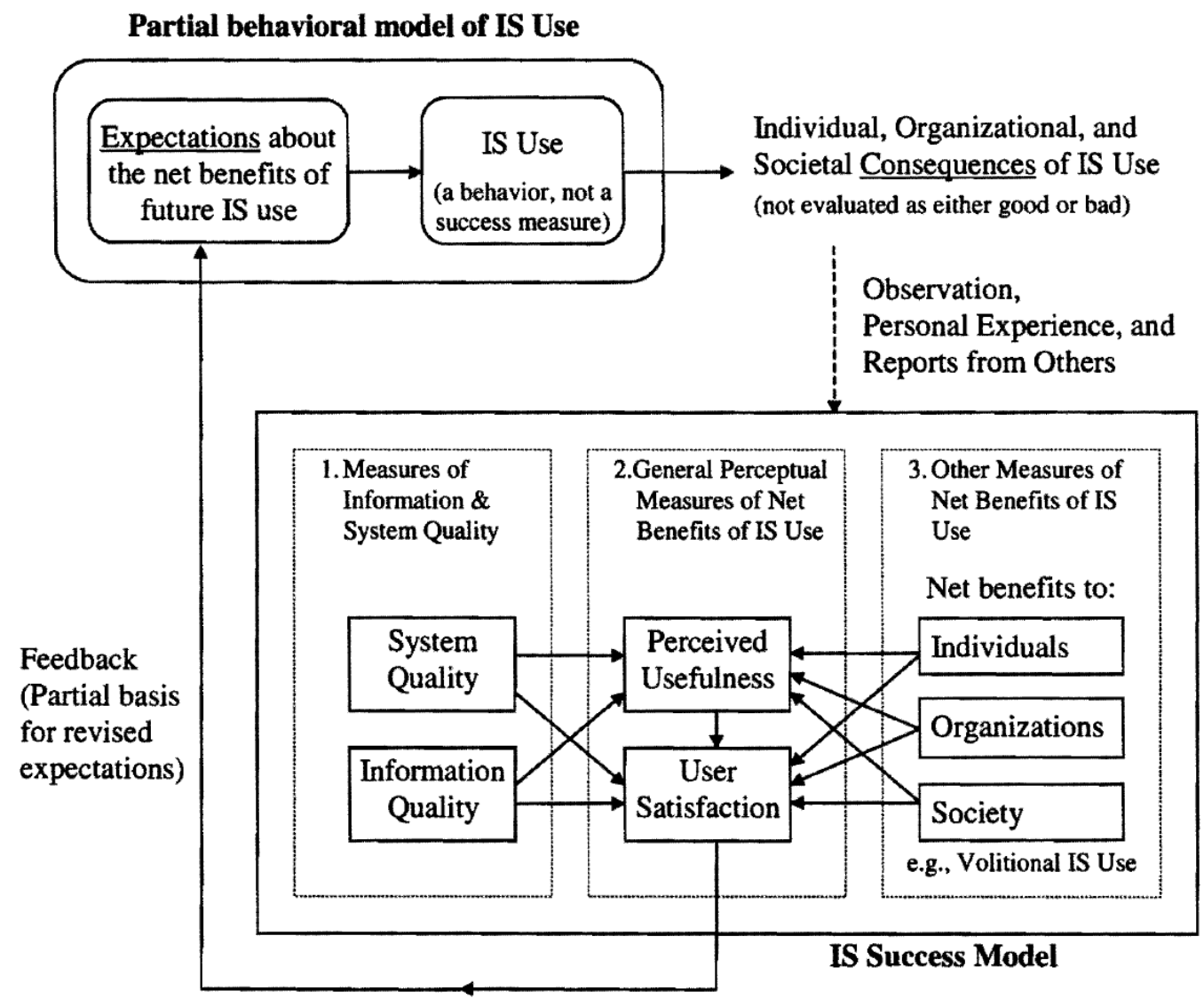

Key:

Rectangular boxes

IS Success model

Rounded boxes

Partial behavioral model of IS Use

Solid-line arrows

Independent (necessary and sufficient) causality

Dotted-line arrow

Influence (not causal, since observer's goals are unknown)

Figure 2.7: Seddon's respecification of the DeLone \& McLean IS Success Model (Seddon, 1997, p. 245) 


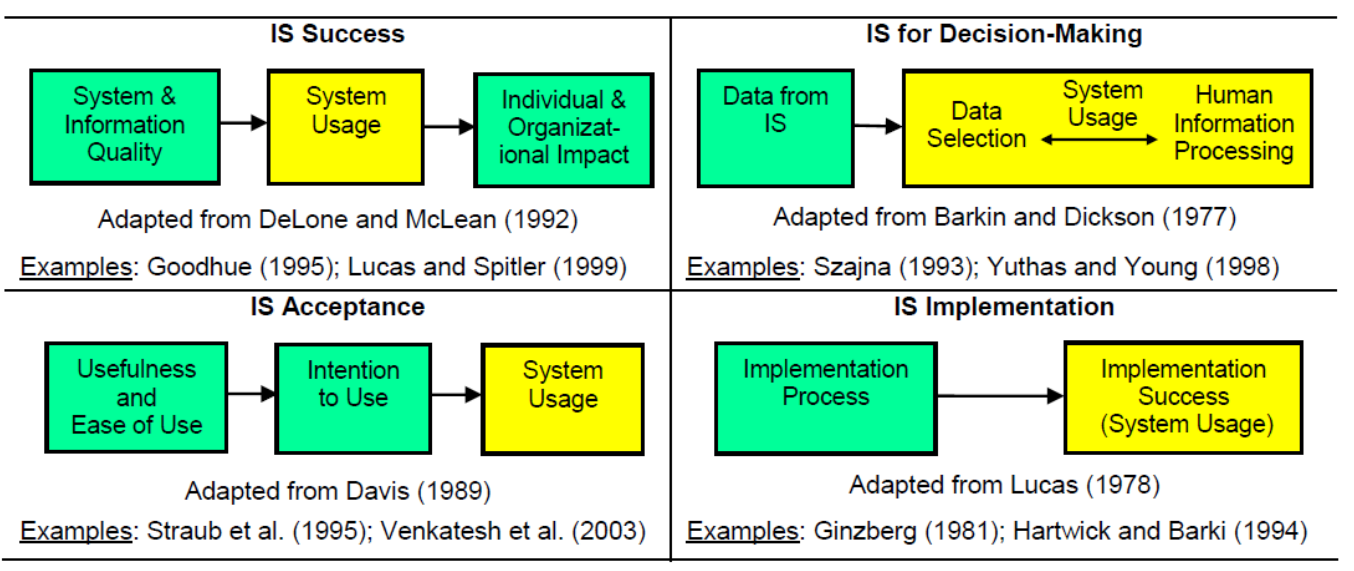

Figure 2.8: Past conceptualizations of the system-usage construct (BurtonJones \& Straub, 2006, p. 2)

Jones \& Straub, 2006, p. 6). As stated in the definition, three interactions are necessary: involving the user, the system, and a task or function. This definition has two main implications. Most importantly, it delineates the construct from similar concepts such as information use (Choo, Bergeron, Detlor, \& Heaton, 2008). The present study is less concerned with whether individuals utilize the information stored in the EDRMS - e.g., to enhance decision-making or reduce risk - and more interested in the antecedent variables that lead to the decision to contribute to the system and store a document and/or a record. Secondly, this definition of system use specifically excludes an evaluation of how well the system is used. As such, this definition further differentiates the construct from other qualifying concepts such as appropriate use (Chin, Gopal, \& Salisbury, 1997) or quality of use (Auer, 1998). Although quality of use is important to the value of the resulting records and their management, the focus of this research remains limited to identifying and measuring the factors connected solely to the intention to use the system in the first place.

Having limited the scope of system use to its location as the dependent variable, it is now important to place 'boundaries' and/or 'modal qualifiers' around the type of system use under consideration (Gregor, 2006). For example, EDRMS-use could include: initial storage, entering of metadata, record workflow, retrieval, versioning, long-term storage, contextual grouping, managing of security, search, etc. 
Ashton and Reed (2011) simplified the EDRMS-use options by considering when and how the users interact with records:

There are two major intersections of users and recordkeeping. The first is putting records into the system, and the second is search and retrieval to find and use existing records. These do not have to work on the same paradigm. Different mechanisms can be constructed to support both (Ashton \& Reed, 2011, p. 7).

To help focus the research, and to avoid excessive attention on individual recordkeeping processes or functions, system use will focus primarily on the act of contribution; however, measurement of the construct will use published measurement items where possible and appropriate and will include additional aspects of use that are specific to electronic recordkeeping systems.

Unfortunately, actual usage data is extremely difficult to obtain from large organizations. Nonetheless, a number of earlier studies have demonstrated that actual usage behavior and behavioral intention are highly correlated (Fishbein \& Ajzen, 1975; Davis, Bagozzi, \& Warshaw, 1989; Dishaw \& Strong, 1999; Turner, Kitchenham, Brereton, Charters, \& Budgen, 2010). In a systematic review of 79 relevant empirical studies, Turner et al. (2010) found that behavioral intention to use was likely correlated with actual use, and this finding supports the relationship initially posited by Fishbein and Ajzen (1975). The implication is that a user's intention to use can be measured and used as a suitable and reliable proxy for their actual system use behavior.

In many of the above system use models, an intention to act also implies unconstrained freedom to choose. However, in practice, constraints such as limited ability, time, environmental or organizational factors, and unconscious habits will limit this freedom (Wilkins et al., 2009b, p. 55).

\subsubsection{Determinants of System Use}

Next to be considered are factors that may affect system use (or rather intention to use). The literature provides a number of conceptual areas (including models and constructs) that warrant attention.

Primarily, an EDRMS is an enterprise-class technology system. Therefore, an individual's willingness to use that technology must be considered on par with any other technology system (see Technology Acceptance below). Secondly, electronic recordkeeping technologies are necessarily used in an organizational context, so social conditions specific to the organization and 
their combined impact on the user are next considered (see Organizational Context on p. 45). Finally, the interpretation required to value electronic records and the process of recordkeeping are considered (see Knowledge Interpretation on p. 50).

\section{Technology Acceptance}

Technology acceptance is an area of IS theory that attempts to model how users come to accept and use a technology. Figure 2.9 reproduces Davis' original Technology Acceptance Model (TAM) (Davis, 1986). TAM is one of the most influential theories in technology systems adoption and use behavior research (Venkatesh \& Davis, 2000; Venkatesh et al., 2003; Wilkins et al., 2009b) and was adapted from Ajzen and Fishbein (1980)'s Theory of Reasoned Action (TRA) (Venkatesh \& Davis, 2000).

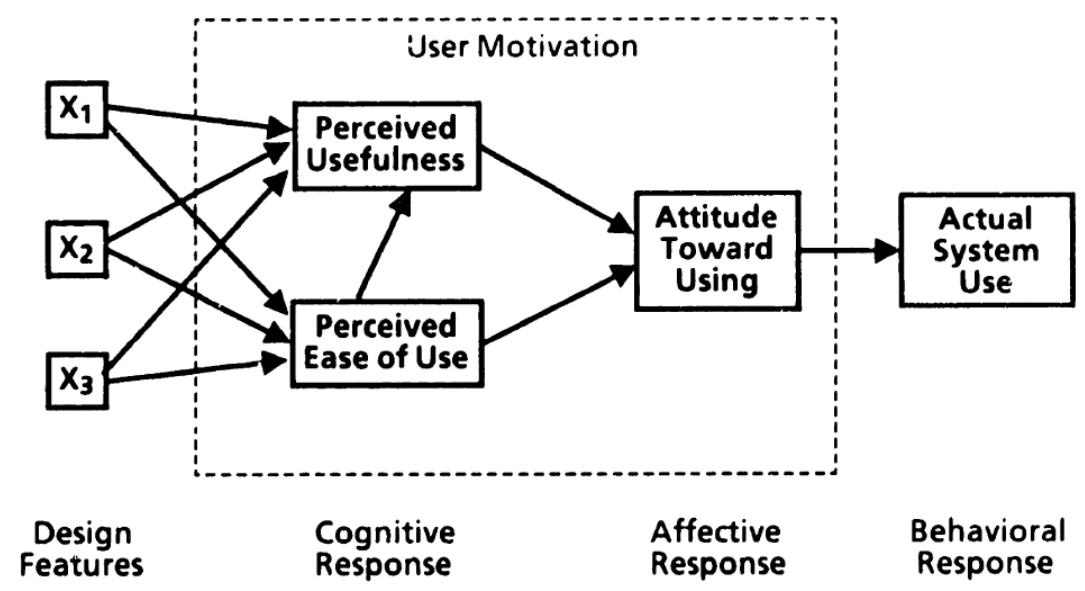

Figure 2.9: The Technology Acceptance Model (TAM) (Davis, 1986, p. 24)

The Technology Acceptance Model is based on the observation that an actual behavior (e.g., system use or 'tool use') is highly correlated with the intention to perform the behavior (Turner et al., 2010; Dishaw \& Strong, 1999; Bagozzi, 1982). Furthermore, the intention to perform the behavior is determined by the user's perception of the technology's usefulness and its ease of use. These two constructs form the basis for one's attitude toward use, which loads onto one's intention to use and, ultimately, use. As a model, TAM consistently explains a large proportion of the variance in use-intentions and ultimately use-behavior across all time frames, with the explained variance regularly approaching 40 percent (Venkatesh \& Davis, 2000, p. 186). 
However, the model is not without its detractors. For example, when commenting on the application of TAM to practical situations as part of an open-ended survey, Dennis commented: "imagine talking to a manager and saying that to be adopted, technology must be useful and easy to use. I imagine the reaction would be 'Duh!' The more important questions are what makes technology useful and easy to use" (Y. Lee, Kozar, \& Larsen, 2003, p. 766).

Nonetheless, this research submits that the ability to identify usefulness and/or ease of use as issues in getting a particular technology accepted will help practitioners to begin to narrow the search for the source of the underlying acceptance problem(s).

In the 1990s, TAM enjoyed a significant amount of attention in the IS research community as other researchers sought to replicate (and validate) Davis's original study and provide additional empirical evidence relating perceived usefulness, perceived ease of use, and system use. This included replication of the original study with improved analysis tools such as test-retest scenarios (Hendrickson, Massey, \& Cronan, 1993) and structural equation modeling (SEM) (Adams, Nelson, \& Todd, 1992) ${ }^{6}$ as well as studies that focused on providing the theoretical underpinning of the relative importance of TAM constructs; that is, perceived usefulness and perceived ease of use (Karahanna \& Straub, 1999).

The results of TAM under SEM scrutiny supported the model's strengths, but also detected some possible weaknesses in several measurement items when used in different contexts. The established TAM measurement items were then studied with more rigor, using newer, sensitive statistical methods. Segars and Grover (1993, p. 525) found that indeed the measurement items did load differently on the two primary TAM constructs under different contexts. They concluded:

Such findings in no way diminish the value of Davis' (1989) original scales or the value of identifying measures that explain technology acceptance. Instead, they challenge the IS community to further explore the nature and specific influences of factors that may alter the "user perception-usage" equation.

In an effort to further explore the "user perception-usage" equation, Venkatesh and Davis created TAM2 - see Figure 2.10 (p. 39) - which was an extension to the TAM model with a focus on additional determinants and

\footnotetext{
${ }^{6}$ For more information on Structural Equation Modeling, see Section 7.4 on p. 162.
} 
modifiers to perceived usefulness (Venkatesh \& Davis, 2000). In 2003, this was followed by a massive reformulation of the technology acceptance model into the Unified Theory of Acceptance and Use of Technology (UTAUT) (Venkatesh et al., 2003). UTAUT is reproduced in Figure 2.11 (p. 41).

In 2007, the Journal of the Association for Information Systems (JAIS) featured a special issue focusing on the theoretical implications of TAM (including versions such as UTAUT). Benbasat and Barki (2007) acknowledged that perceived usefulness and perceived ease of use are influential constructs "almost to the point of certainty"; however, they declared that the "key problem with TAM could be described as a focus on a middle range theory that provides a potentially useful bridge to antecedents and consequences of adoption, but the bridge seems to have become an end in itself" (Benbasat \& Barki, 2007, p. 216). Straub and Burton-Jones (2007) agreed that the focus on TAM constructs has limited research into other aspects of technology acceptance and use, but they took exception to the assertion that TAM was 'unassailable' by pointing out the unresolved risk of common method bias ${ }^{7}$ among the majority of empirical TAM studies. Goodhue (2007) further commented that TAM studies are generally quite static, while business processes and their support systems are naturally undergoing change. This raises the question of "how to design (and redesign) tasks at the same time as designing (and redesigning) information system" (Goodhue, 2007, p. 221), thus highlighting another potential weakness in TAM in that its approach "does not encourage us to think about these larger contexts."

In 2008, Venkatesh returned to TAM (partially in response to the JAIS special issue) with the addition of a new direction of extension into the determinants of perceived ease of use resulting in TAM3 (Venkatesh \& Bala, 2008). TAM3 is reproduced in Figure 2.12 (p. 43).

In the next two sections, the Technology Acceptance Model's two primary determinants of system use constructs, perceived usefulness and perceived ease of use are covered in more detail, including examples of how they have been approached via the various models and analysis techniques referenced above.

Perceived usefulness In the original Technology Acceptance Model (TAM), Davis tested the construct perceived usefulness in two studies and found that

\footnotetext{
${ }^{7}$ Common method bias - or common method variance (CMV) - is "variance that is attributable to the measurement method rather than to the constructs the measures represent" (Podsakoff, MacKenzie, Lee, \& Podsakoff, 2003, p. 879). Steps taken to mitigate the risk of CMV in this research are addressed in Section 4.4.2 on p. 79.
} 
this construct and associated measurement scales showed excellent psychometric characteristics and strong validity. Davis found that perceived usefulness was correlated 0.63 with self-reported system use in the first study and correlated 0.85 in the second study, demonstrating both a strong and significant correlation with system use (Davis, 1989, p. 333).

Davis defined perceived usefulness as:

the degree to which a person believes that using a particular system would enhance his or her job performance (Davis, 1989, p. 320).

Since TAM's original perceived usefulness construct proved to be such a fundamental driver of use-intention, in 2000 Venkatesh \& Davis sought to better understand the determinants of this construct and introduced a number of new antecedent factors to this specific construct, resulting in the TAM2 variance model. This is shown in Figure 2.10.

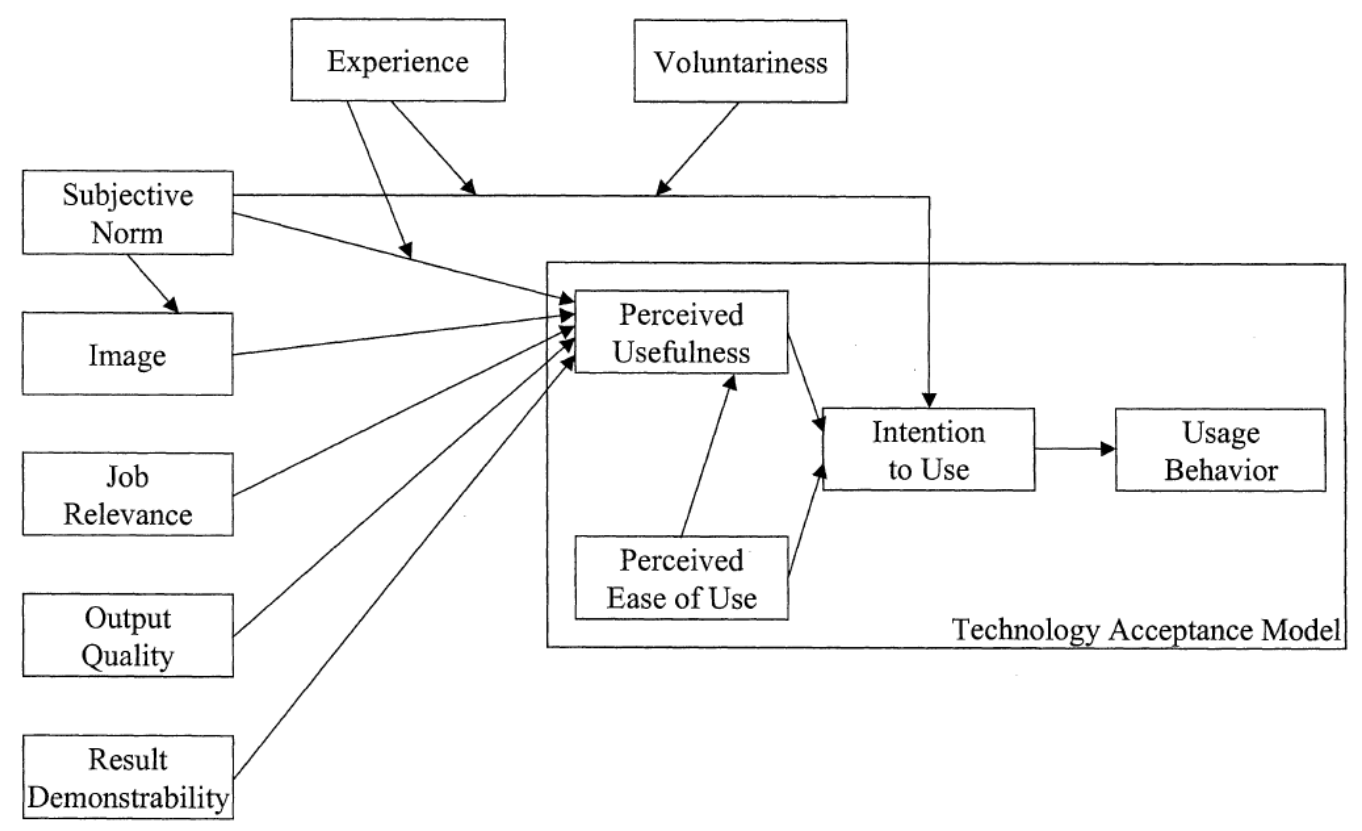

Figure 2.10: TAM2 - extension of the Technology Acceptance Model: a study of Perceived Usefulness (Venkatesh \& Davis, 2000, p. 188)

The addition of the new determinant factors in TAM2 (i.e., subjective norm, image, job relevance, output quality, and results demonstrability) successfully 
explained up to 60 percent of the variance associated with perceived usefulness immediately prior to the introduction of a new technology - i.e., during the pre-use phase as illustrated in Figure 2.5 (p. 31).

However, in their two study groups, the explanatory capability of the TAM2 model fell back to 40 percent and 44 percent (on par with the original TAM model) after only three months of use experience with the technology (Venkatesh \& Davis, 2000, p. 196). This illustrates the decay in the importance of these additional variables as they are replaced with actual experience during the initial-use and continued-use phases of technology adoption. After three months of use, the ability of the TAM2 model to explain intention to use was not appreciably better than the simpler TAM in stabilized continued-use scenarios (Venkatesh \& Davis, 2000, p. 195). Only subjective norm, job relevance, and results demonstrability (and TAM's perceived ease of use) continued to have significant explanatory power.

In 2003, Venkatesh and Davis created the Unified Theory of Acceptance and Use of Technology (UTAUT) model in an attempt to improve on TAM's explanatory value (see Figure 2.11). This new technology acceptance model built on the success of TAM; however, it focused on expanding the definition of the core constructs to encapsulate other similar constructs from the literature and thus deliver on its promise as a 'unifying theory'. Among the reformulated constructs was a newly renamed construct, performance expectancy, to replace and expand upon the original perceived usefulness.

Performance expectancy is conceived of as a slightly broader construct that includes multiple overlapping constructs including: perceived usefulness (Davis, 1989; Davis et al., 1989; Venkatesh \& Davis, 2000); relative advantage (Moore \& Benbasat, 1991); and outcome expectation (Compeau \& Higgins, 1995; Compeau, Higgins, \& Huff, 1999). 


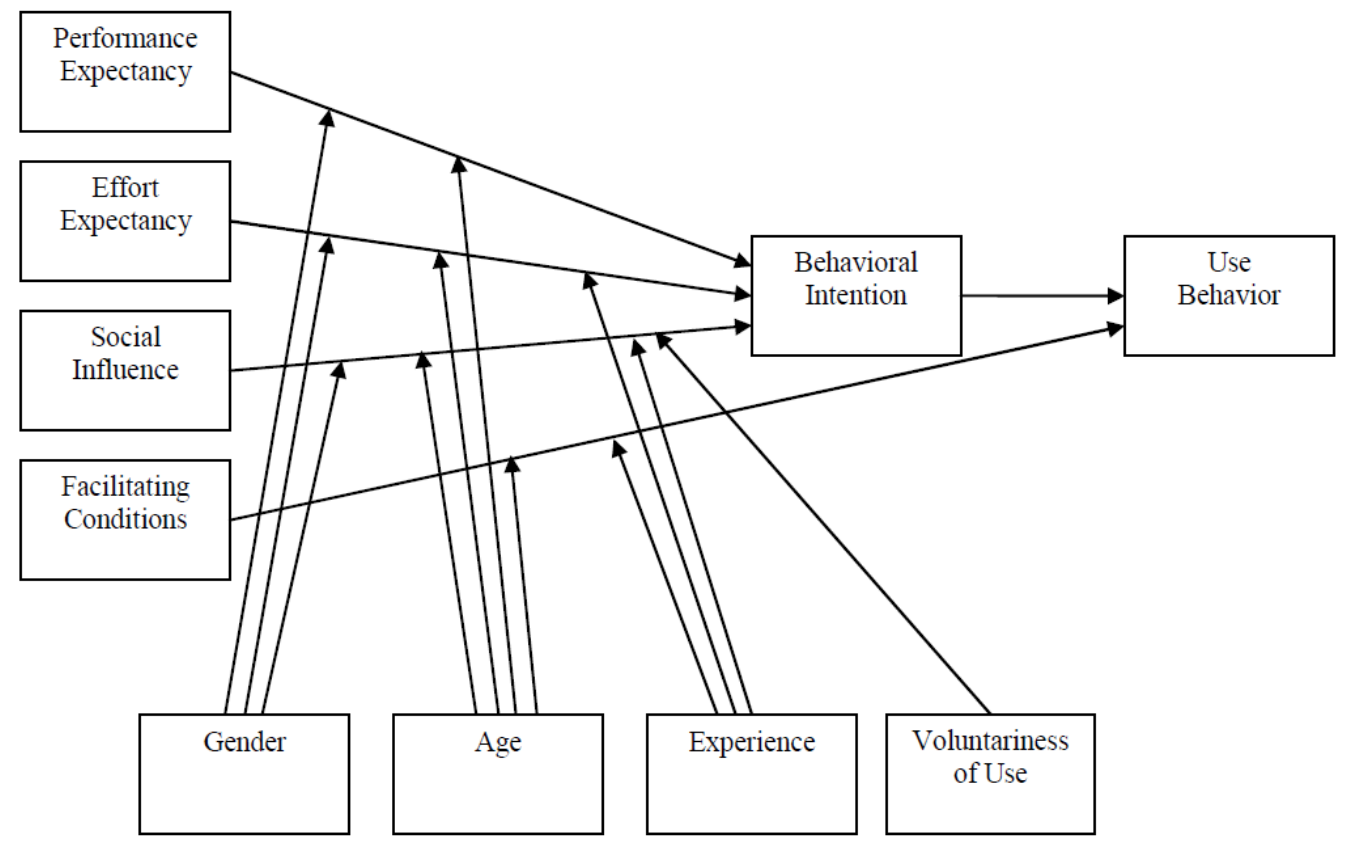

Figure 2.11: UTAUT: Unified Theory of Acceptance and Use of Technology (Venkatesh, Morris, Davis, \& Davis, 2003, p. 447)

Performance expectancy is defined as:

the degree to which an individual believes that using the system will help him or her to attain gains in job performance (Venkatesh et al., 2003, p. 447).

The results of the UTAUT model found that the performance expectancy construct (within each of the individual studies) remained the strongest predictor of intention and "remains significant at all points of measurement in both voluntary and mandatory settings" (Venkatesh et al., 2003, p. 447). Performance expectancy, which encapsulates perceived usefulness, is therefore a candidate for a variance model construct of EDRMS-use and was incorporated into the research model.

Perceived ease of use The second major component of the Technology Acceptance Model is perceived ease of use. Davis defined perceived ease of use as "the degree to which a person believes that using a particular system would be free of effort" (Davis, 1989, p. 320). In his 1989 studies, Davis found 
that perceived ease of use was correlated 0.45 with system use in Study 1 and 0.69 in Study 2 (Davis, 1989, p. 333).

This construct was also broadened and redefined in the 2003 UTAUT model (p. 41), where it was recast as effort expectancy. Like the other UTAUT constructs, effort expectancy is conceived as a slightly broader construct that includes multiple overlapping constructs including: perceived ease of use (Davis, 1989; Davis et al., 1989; Venkatesh \& Davis, 2000); complexity (Thompson, Higgins, \& Howell, 1991); and ease of use (Moore \& Benbasat, 1991).

Effort expectancy is defined as:

the degree of ease associated with the use of the system (Venkatesh et al., 2003, p. 450).

Generally, effort-oriented constructs would be expected to be more relevant to use-intention in the early stages of a new system or behavior, during the period when new process issues represent hurdles to be overcome. And indeed, in the original UTAUT research, effort expectancy was significant, but only during the first time-period (post-training), becoming non-significant over periods of extended and sustained usage.

The UTAUT model, with all of its constructs and mediating factors, accounted for an impressive 70 percent of the variance in behavioral intention (Venkatesh et al., 2003, p. 467). It has since received a large following, including replication and even model extensions (for example: Wang, Wu, \& Wang, 2009; Verhoeven, Heerwegh, \& De Wit, 2010). However, UTAUT is not without its criticisms; for example, van Raaij and Schepers pointed out that:

UTAUT's high $R^{2}$ is only achieved when moderating the key relationships with up to four variables (gender, age, experience and voluntariness) in order to yield more significant coefficients. This makes the model less parsimonious than TAM and TAM2. More importantly, we find the grouping and labeling of items and constructs problematic, especially for facilitating conditions and social influence (van Raaij \& Schepers, 2008, p. 840).

In 2008, Venkatesh and Bala returned to the original TAM2 model (which had focused on the determinants of perceived usefulness), and expanded the model with an increased focus on the determinants of perceived ease of use, thus creating TAM3. See Figure 2.12 (p. 43). 


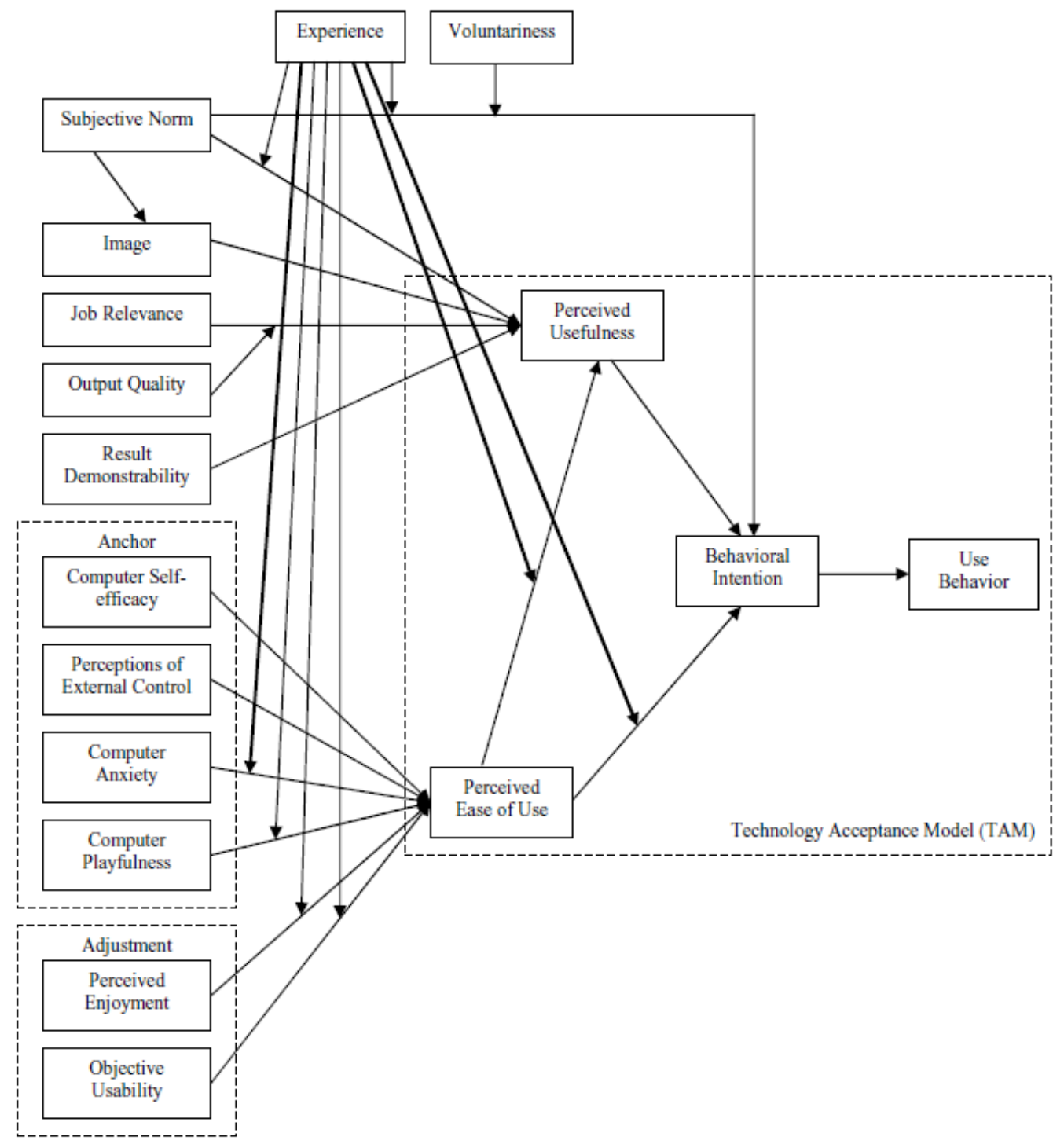

Figure 2.12: Technology Acceptance Model 3 (TAM3) (Venkatesh \& Bala, 2008, p. 280) 
This model successfully enhanced the understanding of the perceived ease of use construct and provided a level of richness and detail that would allow the model to be used in practice to design interventions; however, even the authors acknowledged that TAM3 is far from a parsimonious model (Venkatesh \& Bala, 2008, pp. 301-302).

In the context of electronic recordkeeping systems, an organization's standard operating procedures generally mandate the capture of records and working documents in the system. However, even in mandatory-use scenarios, the use of the system (i.e., entering the required metadata, deciding on the appropriate classification, etc.) continues to be at the discretion of the user. The user must weigh up the effort to use the recordkeeping system against alternative storage options, such as saving the document locally or emailing it without submitting it to the system (Bruce, 2005). As such, it is likely that perceived ease of use issues will remain a significant factor in system use long after implementation and well into stabilized continued-use situations.

The long-term significance of perceived ease of use/effort expectancy is supported by a recent application of UTAUT to electronic medical records systems, where ease of use remained significant in all timeframes (Venkatesh, Sykes, \& Zhang, 2011, p. 7). This is also expected to remain a significant factor in recordkeeping systems, especially for occasional-use individuals, who may feel as if they need to relearn the system at each use (having forgotten how it works in between uses) (Kim \& N. Malhotra, 2005).

Due to its more inclusive and slightly broader definition, the UTAUT construct effort expectancy is another candidate for a variance model construct as applied to electronic recordkeeping system use. Therefore, it was also incorporated into the research model.

In conclusion, TAM-derived constructs work well, but they are not without limitations. For example, TAM constructs focus primarily on perceived personal benefits and hence fail to consider organizational benefits, except through the lens of the individual (Hardgrave \& Johnson, 2003, p. 326). In addition, other studies have noted that some national cultures are more sensitive to user perceptions than others, and therefore the magnitude of measures of the TAM/UTAUT constructs (e.g., usefulness/performance expectancy and ease of use/effort expectancy) may (Venkatesh \& Zhang, 2010) or may not (McCoy, Galletta, \& King, 2007, p. 89) be universal. Finally, as the use of technology increasingly becomes ubiquitous in all aspects of a user's life, the context of that use (e.g., as a leisure activity) will impact the strength of measures such as performance expectation (which assumes a task-driven orientation) as opposed to other factors such as a technology's perceived 
enjoyment (Van der Heijden, 2004) or hedonic motivation (Venkatesh, Thong, \& Xu, 2012, p. 161), which can provide alternative behavioral motivations for continued use.

Having considered the factors that may impact a single user and their use of a technology, we will now examine factors that account for the situation where users interact with a technology and with each other.

\section{Organizational Context}

Electronic recordkeeping systems (as discussed in Section 2.4.2) are primarily used within an organizational context. Whereas the original TAM constructs target an individual's perceptions toward a particular technology, EDRMS are necessarily used within an organization and therefore within a social structure/ context, where environmental factors, particularly interactions with other individuals and social/cultural norms, can affect an individual's perceptions of the technology. Furthermore, use of technologies in an organizational context can also unbalance existing power interactions within the organization (Kling, 1980), further highlighting the importance of social interactions and norms on attitudes toward a recordkeeping technology.

Lee (2014) went so far as to describe TAM and UTAUT as 'incomplete models' because they fail to take into account the social structure(s) in which the user operates. Giddens' Structuration Theory (discussed in Section 2.3.1), identifies three main social interactions that are used in the constitution of a society: communication, power, and sanction (see Figure 2.2, p. 20). These interactions guided the selection of additional construct candidates for the research model.

Social Influence Fishbein and Ajzen (1975)'s Theory of Reasoned Action (see Figure 2.13) introduced the theory that a behavioral intention was determined in part by one's internal attitude toward the behavior and in part by the external social pressure from others concerning that behavior. Their early model introduced a construct that sought to measure the external social influences associated with a particular behavior which they called the subjective norm. This was defined as: "the person's perception that most people who are important to him think he should or should not perform the behavior in questions" (Fishbein \& Ajzen, 1975, p. 302).

The subjective norm became a widely used construct. It was applied directly to technology use in Ajzen's theory of planned behavior (Ajzen, 1991). Taylor and Todd also used the construct in their decomposed theory of planned 


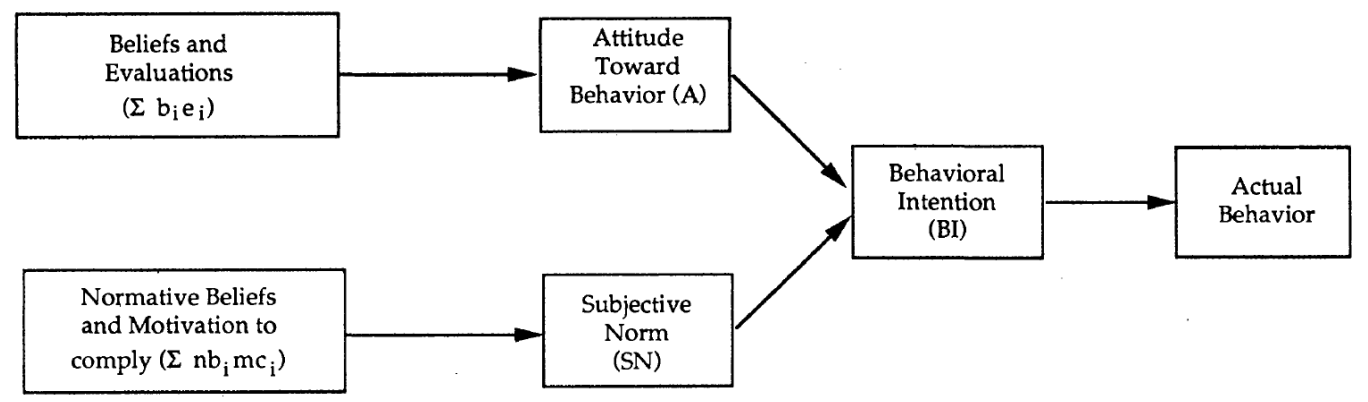

Figure 2.13: Fishbein and Ajzen's Theory of Reasoned Action (Davis, Bagozzi, \& Warshaw, 1989, p. 984)

behavior (Taylor \& Todd, 1995b), where they found attitude, subjective norm, and perceived behavioral control each contribute to behavioral intention.

The subjective norm was later introduced to the TAM constructs by Schepers and Wetzels (2007), where it was hypothesized to have effects on both intention to use and perceived usefulness. See Figure 2.14 (p. 47). In mandatory-use (and sometimes voluntary-use) situations, the compliance effect is often observed, where people choose to perform an action - i.e., to exhibit intention to use when one or more important referents say they should, even though they do not like or believe in it (Schepers \& Wetzels, 2007, p. 91). This is in alignment with Giddens' sanction interaction. The second effect is internalization and it is theorized to affect perceived usefulness. In this situation, there is a human tendency "to interpret information from important others as evidence about reality" (Schepers \& Wetzels, 2007, p. 91). These effects are in alignment with Giddens' sanction and communication interactions.

As the use of electronic recordkeeping systems in public sector organizations is generally reliant on the behavior of end users, the compliance effect and the internalization effect of the subjective norm on the intention to use become particularly salient. In Schepers and Wetzels (2007)'s meta-analysis of published quantitative TAM studies that incorporated the subjective norm, it was found that the subjective norm contruct exhibited a large impact on behavioral intention. In addition, the impact of the subjective norm on perceived usefulness was also large and significant. The explained variance $\left(R^{2}\right)$ associated with intention to use approached 0.48 , which was an improvement over TAM without incorporating the social norm.

In an earlier study of the adoption of mandated-use systems in the banking industry, the addition of the subjective norm to TAM in mandatory adoption 


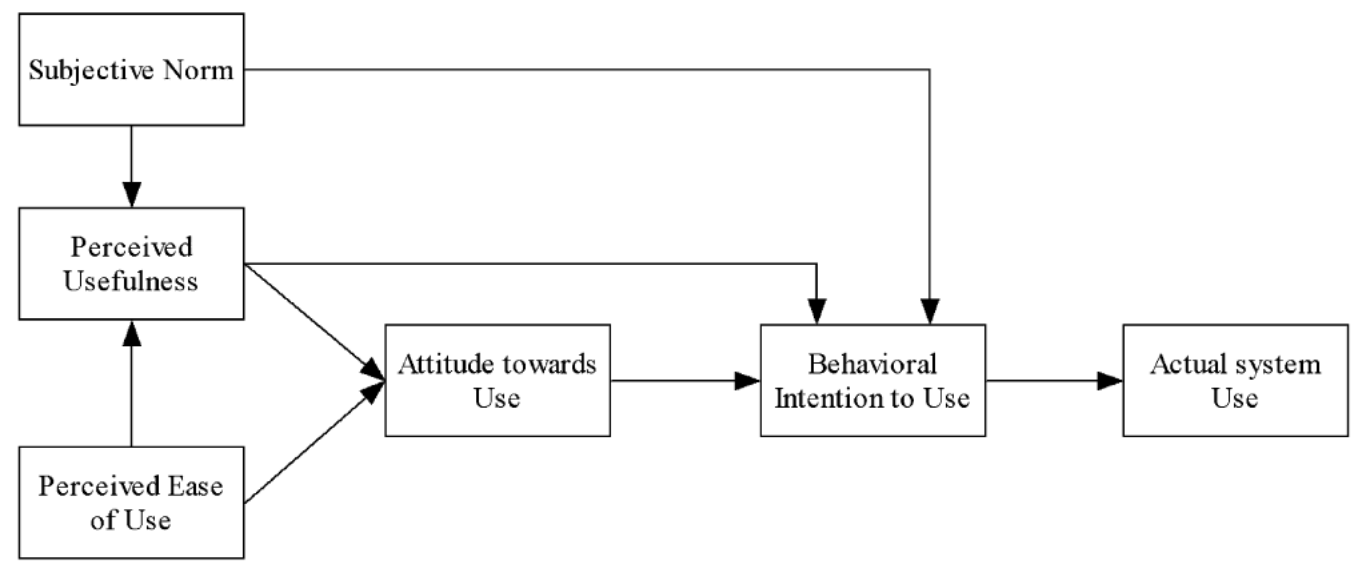

Figure 2.14: Addition of the Subjective Norm to the Technology Acceptance Model (Schepers \& Wetzels, 2007, p. 98)

scenarios was also found to increase the explained variance associated with intention to use (this time, with an $R^{2}$ of 0.55 ) (Brown, Massey, MontoyaWeiss, \& Burkman, 2002).

The subjective norm construct was later recast as social influence in the UTAUT model (p. 41). As with other UTAUT constructs, social influence was defined to be a slightly broader construct made up of similar overlapping constructs from the literature including: subjective norm (Fishbein \& Ajzen, 1975; Ajzen, 1991; Mathieson, 1991; Taylor \& Todd, 1995a, 1995b), social factors (Thompson et al., 1991), and image (Moore \& Benbasat, 1991).

Social influence is defined as:

the degree to which an individual perceives that important others believe he or she should use the new system (Venkatesh et al., 2003, p. 451).

When tested in the UTAUT model, social influence was found to be significant in explaining intention to use - again, particularly when use was mandated (Venkatesh et al., 2003, pp. 451-452). In true mandatory-use situations, users are generally forced into a situation where they either use the technology or they may engage in alternative (e.g., destructive) behaviors, and this may or may not be intentional (Brown et al., 2002). Use of EDRMS in public sector organizations is often legislatively and operationally mandated, but the extent of use is still at the discretion of the user. Thus, social influence would be expected to remain a significant factor in their use-intention, and is another candidate construct for use in the research model. 
Social influence would be expected to vary depending on local cultural norms. In a comparison of Western versus non-Western studies, the subjective norm had a slightly larger effect on intention-to-use in Western studies (Schepers \& Wetzels, 2007, p. 100); and in a US versus China study, UTAUT (which included social influence) was found to 'work differently' in China, where there are generally stronger collective pressures to conform (Venkatesh \& Zhang, 2010).

In considering Giddens' Structuration Theory, it is argued that social influence incorporates two of the three main societal interactions. The compliance effect satisfied Giddens' sanction interaction, while internalization satisfied the communication interaction. This leaves the final interaction: power.

Perceived Power Security Knowledge management systems (KMS), which are similar to electronic document management systems, have the capability of incorporating Giddens' structure of domination via the interaction of power (refer to Figure 2.2, p. 20). In this context, two kinds of power over resources are manifested: authoritative - power extending over people; and allocative power extending over objects or materials (Orlikowski, 1992, p. 405).

EDRMS technologies (particularly when newly implemented) have the potential to disrupt both. First, by increasing the transparency of individuals' contributions to the EDRMS and making them accessible to the organization, managers and peers can access and potentially judge the work of individuals. Second, by centralizing recordkeeping into an EDRMS, individuals with allocative power over legacy systems and/or control over individual documents may perceive a loss of power to those who administer and manage the EDRM system. Furthermore, these feelings may become entrenched and be exhibited long into the continued-use phase (p. 31).

The ability of information technology systems to centralize and change power ownership within organizations has been studied for some time (Kling, 1980). In terms of power shifts (i.e., loss of security) associated with the introduction of new technology systems, the more negative the expectations of employees, the more negative are their reactions, including apprehension, anxiety, stress, and fear (Martinko, Henry, \& Zmud, 1996). The intensity of these emotions can affect one's intention to use a technology, as well as impacting the technology's perceived ease of use (Hackbarth, Grover, \& Yi, 2003). This continuum of emotion has been captured in the construct: perceived power security. 
Perceived power security is defined as:

the degree to which a person believes that using a particular system will be free from threats of insecurity regarding loss of power and influence (Ong, Lai, Wang, \& Wang, 2005, p. 3).

In their study, which focused on the implementation and subsequent acceptance of a knowledge management system, Ong et al. (2005) combined the traditional TAM model constructs with both the subjective norm and perceived power security. See Figure 2.15. They found the explained variance of their model to be much higher than TAM alone, explaining 61 percent of the behavioral intention to use (Ong et al., 2005, p. 6).

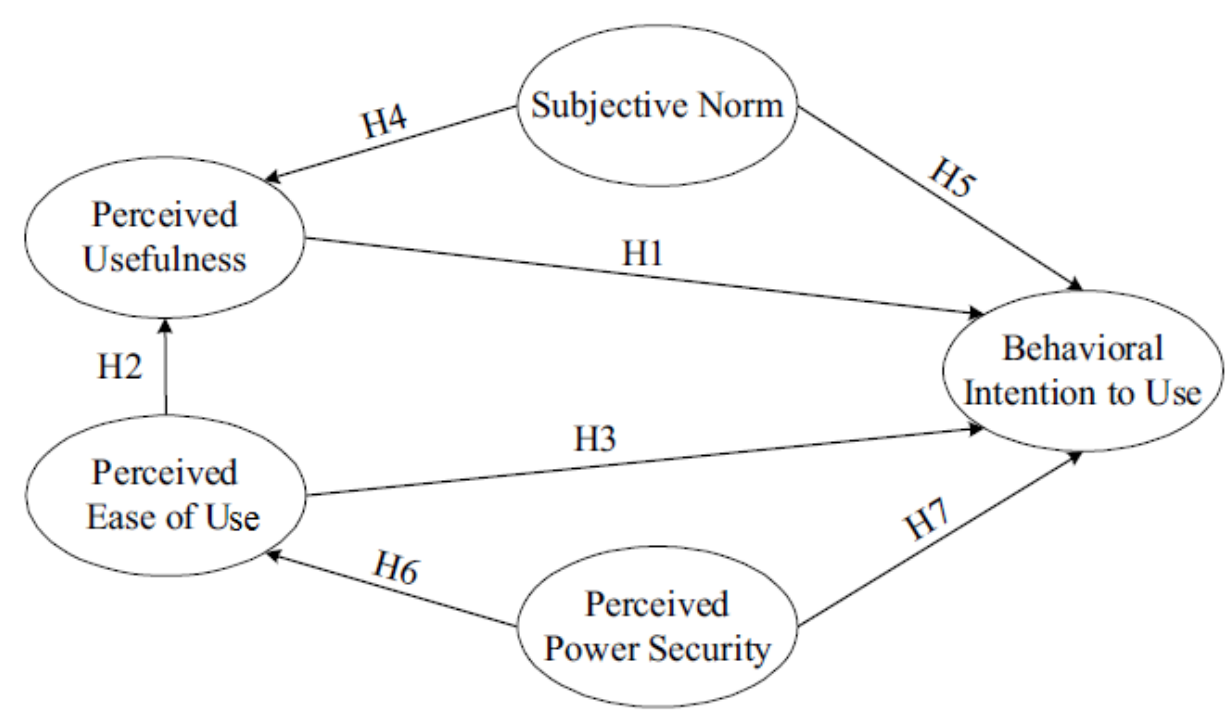

Figure 2.15: Addition of perceived power security and the subjective norm to the Technology Acceptance Model (Ong, Lai, Wang, \& Wang, 2005, p. 6)

In terms of satisfying Giddens' three structurational interactions, the subjective norm/social influence (with its ability to satisfy both the communication and sanction interactions) and perceived power security (with its ability to satisfy Giddens' power interaction) appear to cover all three.

Thus far, we have addressed technology acceptance constructs related to use of the system and organizational context constructs representing the social context of its use. In the next section, we will investigate how users perceive and interpret the value of electronic records (and the process of 
recordkeeping) and how the importance assigned to these records (and the information they contain) may impact on the users' willingness to use the system.

\section{Knowledge Interpretation}

EDRMS require end users to add contextual metadata when entering their documents into the system or when declaring those documents as records. As discussed in Section 2.2, the creation and tracking of these documentspecific contextual metadata are fundamentally what differentiates documents from records; however, this prerequisite necessarily requires additional understanding and effort on the part of end users. Therefore, the contribution of documents into an EDRMS requires both a user's willing participation and their interpretation as to what is record-worthy. Although there is continuing research into how to remove the need for voluntary participation in the capture of contextual recordkeeping metadata (Bailey, 2007, 2009), the need for user participation is likely to remain, as will the challenges involved in obtaining and maintaining the participation of end users (Kettunen \& Henttonen, 2010).

J. Klein, Connell, and Jasimuddin (2007), while reviewing the organizational memory literature, ${ }^{8}$ noted that the literature predominantly paints a picture of organizational memory as a repository that is essential for organizational effectiveness. However, the authors also note that depending on the user's frame of reference, retention of detailed documents and records is not necessarily an unqualified good (J. Klein et al., 2007). For example, organizations with a strong knowledge of previous events and solutions may, to their detriment, be less likely to try new and creative solutions. Thus, users may view the value of records differently, depending on how an organization uses and interprets that repository of information, experience, and knowledge. In other words, the value that an end user places on their organization's records repository is expected to influence their willingness to participate in recordkeeping activities.

The quantification of the term value becomes more complicated when one acknowledges that it can have different meanings for different people. Examples of different types of value include: utility, social significance, emotional, and spiritual (Boztepe, 2007), with measurement scales including continuums

\footnotetext{
${ }^{8}$ Organizational Memory refers to several structures and mechanisms that store and reflect previous events and decisions within an organization (including the structures consisting of records and documents). See Walsh and Ungson (1991).
} 
such as extrinsic/intrinsic value, active/reactive value, and self-oriented/otheroriented measures of value (Holbrook, 1999). In order to measure the value of records, it is important to locate and define potential sources of value in the mind of the user, particularly as it applies to information and knowledge.

To help explore these core value assumptions, J. Klein et al. (2007) looked to a four-paradigm framework adapted for knowledge management by Schultze and Stabell (2004), which was in turn based on a framework developed by Burrell and Morgan in 1979.

Burrell and Morgan had created a two-dimensional framework to explore sociological paradigms in organizations. Their ontological dimension (subjectivity to objectivity) was combined with a social order dimension (sociologies of regulation to radical change), and thereby created a $2 \times 2$ grid of sociological paradigms that the authors labeled functionalist, interpretivist, radical structuralist, and radical humanist paradigms (Burrell \& Morgan, 1979).

In 1996, Deetz recast Burrell and Morgan's framework's social order dimension to instead contrast consensus and dissensus within the organization (Deetz, 1996). Consensus reflects a social order based on trust and the common good, where science and/or knowledge is seen as neutral and a 'hegemonic order' is seen as the natural state. In contrast, dissensus reflects a social order based on suspicion and conflict of interest, where science, knowledge, and the 'current order' is seen as heavily politicized (Schultze \& Stabell, 2004).

Deetz also argued that the ontological dimension (subjectivity to objectivity) in the original framework was 'untenable and meaningless' and has more political than descriptive value (Schultze \& Stabell, 2004). In searching for a suitable replacement continuum, Schultz and Stabell changed Burrell and Morgan's original ontological dimension to an epistemological dimension borrowed from Giddens' Structuration Theory, adopting dualism and duality as the two main world views encompassing information and knowledge.

Schultze and Stabell refer to their final framework (see Figure 2.16) as discourses of organizational knowledge (rather than paradigms) "in order to highlight that each of them is plagued by internal debates, that the edges between the worldviews are not well demarcated, and that debates in one world view influence debates in the others" (Schultze \& Stabell, 2004, p. 555). Schultze and Stabell's knowledge discourses include the neofunctionalist discourse, constructivist discourse, critical discourse, and the dialogic discourse. These are explained in more detail below. 


\begin{tabular}{|c|c|c|}
\hline & Duality & Dualism \\
\hline Dissensus & $\begin{array}{l}\text { Dialogic Discourse } \\
\text { Metaphor of Knowledge: discipline } \\
\text { Role of Knowledge in } \\
\text { Organizations: deconstruction of } \\
\text { totalizing knowledge claims, } \\
\text { creation of multiple knowledges } \\
\text { Theories: post-structuralist } \\
\text { theories, feminist theories, } \\
\text { postmodern theories }\end{array}$ & $\begin{array}{l}\text { Critical Discourse } \\
\text { Metaphor of Knowledge: power } \\
\text { Role of Knowledge in Organizational } \\
\text { Underclass: reformation of social } \\
\text { order } \\
\text { Theories: labour process }\end{array}$ \\
\hline Consensus & $\begin{array}{l}\text { Constructivist Discourse } \\
\text { Metaphor of Knowledge: mind } \\
\text { Role of Knowledge in } \\
\text { Organizations: coordinating action, } \\
\text { shared context, recovery of } \\
\text { integrative values, generation of } \\
\text { understanding } \\
\text { Theories: structuration theories, } \\
\text { theories of practice, sensemaking, } \\
\text { actor network theory }\end{array}$ & $\begin{array}{l}\text { Neo-Functionalist Discourse } \\
\text { Metaphor of Knowledge: asset } \\
\text { Role of Knowledge in Organizations: } \\
\text { progressive enlightenment, } \\
\text { prediction, reduction of uncertainty, } \\
\text { optimal allocation of resources } \\
\text { Theories: resource-based view of } \\
\text { firm, transaction cost theory, } \\
\text { information processing theory, } \\
\text { contingency theories }\end{array}$ \\
\hline
\end{tabular}

Figure 2.16: The four discourses of knowledge management research (Schultze \& Stabell, 2004, p. 556) 
Neo-functionalist (Dualism/Consensus) In this discourse, "knowledge is viewed as an asset and the role of knowledge is to progress individuals, organizations and society to the ideal state of enlightenment (or competitive advantage)" (Schultze \& Stabell, 2004, p. 557). In the field of organizational memory and recordkeeping research and practice, the neo-functionalist discourse is arguably the 'default' discourse (J. Klein et al., 2007). However, it is one that is not universally shared by all end users, and it is necessarily the end users who have replaced the traditional 'records clerks' in taking responsibility for contributions to electronic recordkeeping repositories.

Constructivist (Duality/Consensus) The constructivist discourse is concerned with "integrating knowledge and coordinating collective action in organizations, which are conceptualized as systems of distributed knowledge" (Schultze \& Stabell, 2004, p. 558). Adherents of this discourse believe that knowledge cannot be separated from its application. As such, J. Klein et al. (2007) suggest an emerging picture of a Markovian organization. Markov theory is a mathematical theory that "concerns events that are conditional upon those that [immediately] precede them and affect those that come after" (Schwarz, 2002). For individuals with this view of knowledge, the information needs required to solve a current problem are sought in the present, not from the past. Furthermore, any knowledge not required by the organization is simply abandoned and lost to the organization. In other words, knowledge "is integrated with the activities that the organization [currently] undertakes" (J. Klein et al., 2007). In organizations with users who predominantly adhere to the constructivist discourse, efforts to create and maintain physical repositories for organizational memory (e.g., EDRMS) would be seen as having little value or impact on organizational effectiveness. In their view, organizational memory is limited to "knowledge in use: working knowledge" (J. Klein et al., 2007).

The neo-functionalist and constructivist discourses are arguably the most common views found in healthy and successful organizations (due to their focus on the consensus social order dimension). The final two discourses are less common, and primarily occur in organizations with social orders that are categorized as dissensus. These include:

Critical (Dualism/Dissensus) The critical discourse can be categorized as an almost anti-organizational discourse in that it often: "aligns itself with the interest of the workers against management. It regards knowledge as 
an entity separate from the individual knower and knowledgeable action [... with the risk that knowledge can be] extracted from one social grouping and appropriated by another, thus becoming a means of exercising power and control" (Schultze \& Stabell, 2004, p. 559). Adherents view knowledge as having value and capable of being stored (and as such, are similar to neofunctionalists). However, J. Klein et al. (2007) go further and suggest that this discourse sees 'objectified knowledge' as a source of power in an organization with dissensus, and point out that the possessor of that knowledge can either use it to preserve the organization or destroy it through, for example, knowingly maintaining outdated or dysfunctional practices (J. Klein et al., 2007).

Dialogic (Duality/Dissensus) The final discourse type views knowledge within the concept of duality in an organization typifying dissensus. Adherents of the dialogic discourse adopt "a Foucauldian perspective [that] contends that knowledge and power are inextricably intertwined giving rise to the concept of power-knowledge" (Schultze \& Stabell, 2004, p. 560). In this view, those with power-knowledge are likely to use it to further their own interests, which is a common goal in highly political environments.

The main difference in the above ontological perspectives could be argued to be the perceived value-duration of a particular knowledge artifact. These time-slices of a record's perceived value-duration can again be considered in light of Giddens' structuration theory (Section 2.3.1) and his 'planes of temporality': durée (the timeframe of day-to-day life), dasein (the human lifespan), and the longue durée (the timeframe of social institutions).

Those adhering to a neo-functionalist or critical knowledge discourse see all records as potential long-term 'assets' that are worthy of keeping and managing for use in the future (with the value-duration of the knowledge artifact extending to the dasein and longue durée temporal planes). However, an individual's willingness to share that knowledge or participate in an organizational recordkeeping activity is strongly influenced by the power politics of the organization - i.e., whether the organizational culture is primarily categorized as consensus or dissensus. It can thus be argued that even if an individual believes that a particular knowledge artifact has value and would 'normally' be willing to participate, this willingness to participate is contingent on the organizational political context.

In contrast, those adhering to the constructivist or dialogic knowledge discourse perceive the same knowledge artifacts as supporting the current 
'working knowledge' only. Even in the most supportive of consensus-driven organizations, these individuals would still see little or no value in making efforts to formally retain documents and/or records beyond their current application. In dissensus organizations, these individuals may even perceive centralized efforts to systematize and store this 'low value' knowledge as a form of conspiracy and a developing threat against the workers - further steeling their intention not to participate.

\subsection{Chapter Summary}

In this chapter, the literature was explored to set the context and seek out suitable conceptual areas to address the research question: What factors influence a user's intention to use an electronic recordkeeping system?

Primarily, an EDRMS is an enterprise-class technology system. Therefore, an individual's willingness to use that technology (i.e., technology acceptance) must be considered on par with any other technology system. Davis et al. (1989)'s Technology Acceptance Model (TAM) has two primary constructs that have proven over time to be a consistent predictor of system use. The user's perceived usefulness and perceived ease of use were found to explain a significant portion of behavioral intentions, and those intentions were found to drive system use. Venkatesh et al. (2003)'s subsequent UTAUT model redefined the constructs as performance expectancy and effort expectancy, thereby completely capturing the original TAM constructs and attempting to encompass and align similar ones from the literature. The slightly broader UTAUT constructs will be favored over the TAM constructs when used in the conceptual research model.

The second consideration is that electronic recordkeeping systems are implemented across an organization for shared use by its employees, and so an individual's perceptions of a system's usefulness is likely influenced by the social context in which it is being used (i.e., organizational context). Schultze and Stabell (2004)'s categorization of four knowledge management discourses highlights the importance of the political climate and culture, particularly on the power-politics continuum of consensus versus dissensus organizations. Giddens (Section 2.3.1) named three main interactions between individuals in the act of structuring their society: communication, power, and sanction. In terms of the potential impact of the organizational context on an individual's beliefs regarding an EDRMS, the subjective norm (recast by UTAUT as social influence) has been selected for its ability to represent interactions with 
fellow employees, covering both of Giddens' communication and sanction interactions. Perceived power security was selected to address Giddens' final interaction: power.

An approach to the final consideration of how individual's value electronic records and recordkeeping (i.e., knowledge interpretation) is suggested as a result of the second half of Schultze and Stabell (2004)'s knowledge management discourses. In addition to the power-politics continuum of consensus and dissensus, Schultze and Stabell also included a knowledge interpretation dimension, with some users believing that stored knowledge can be an 'asset' that has a lasting value that can be applied in future situations, whereas other users believe that knowledge can only have value while in use, as working knowledge. Accordingly, it would appear that only one of Schultze and Stabell's four knowledge management discourses is conducive to the willing use of EDRMS: that of the neo-functionalists, who both believe knowledge can and should be stored and who also exist in an organizational context that encourages and supports that belief.

With these three categories of the determinants of EDRMS-use derived from the literature, the focus now moves to combining these constructs and developing the conceptual research model. 


\section{Chapter 3}

\section{Conceptual Research Model}

\section{Contents}

3.1 Chapter Overview . . . . . . . . . . 57

3.2 Technology Acceptance Factors . . . . . . 58

3.3 Organizational Context Factors . . . . . . . 60

3.4 Knowledge Interpretation . . . . . . . . 62

3.5 Chapter Summary . . . . . . . . . . . . 64

\subsection{Chapter Overview}

In this chapter, a conceptual research model that includes the hypothesized relationships between its constructs is created to address the research objectives; namely, to identify the factors that influence a user's intention to use an electronic recordkeeping system; and, once identified, to determine their relative importance and influence.

A review of the literature identified three primary sources of factors that may be considered to influence EDRMS use. These sources are grouped into technology acceptance factors, organizational context factors (including constructs that measure a user's localized socio-political climate), and knowledge interpretation factors, including a new construct: the perceived value of records.

Based on the literature, a conceptual research model was developed to more fully explore and relate the factors associated with the use of electronic recordkeeping systems. See Figure 3.1. Each of the conceptual research model's 
constructs and their hypothesized relationships are more fully described in the following sections.

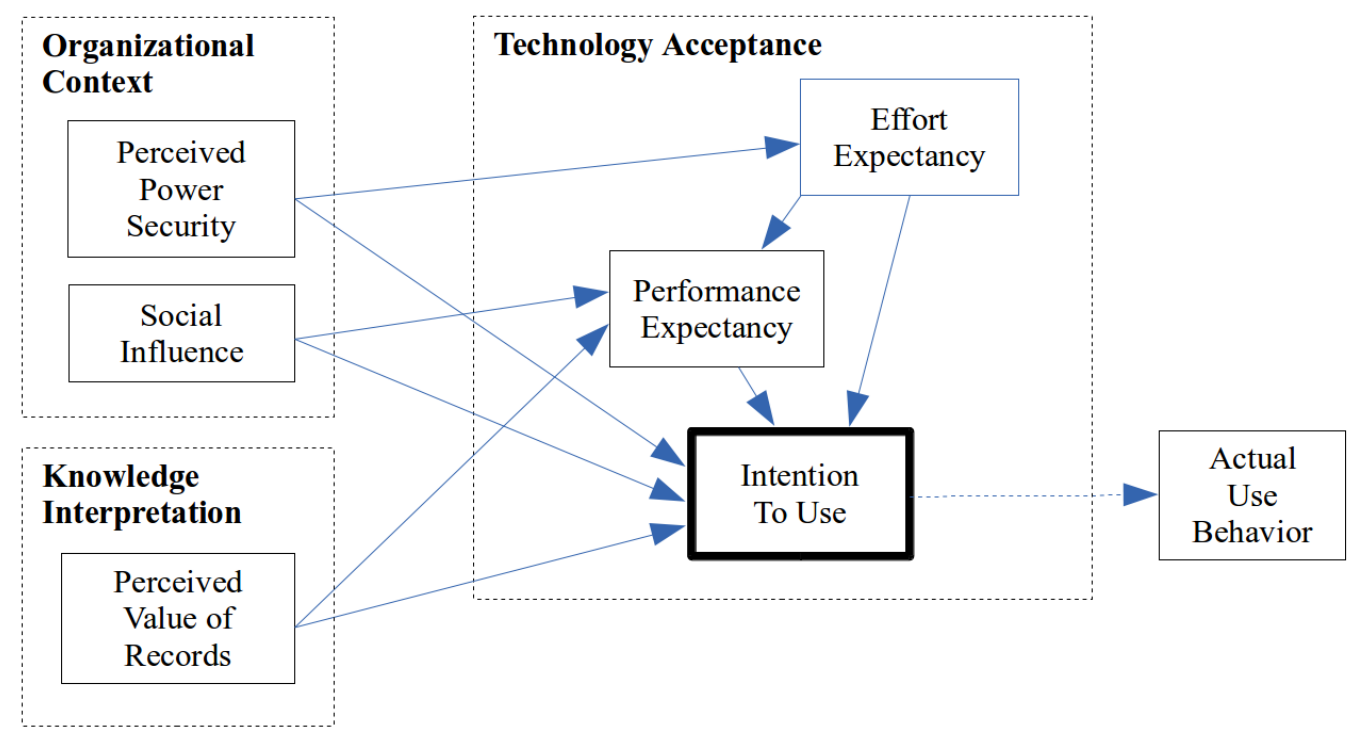

Figure 3.1: A conceptual research model combining the literature with selected research constructs to explain the intention to use an EDRMS

\subsection{Technology Acceptance Factors}

The dependent variable of the research model is intention to use (as a proxy for systems use). At the core of the conceptual research model is Davis et al. (1989)'s Technology Acceptance Model (TAM) and its ability to explain aspects of the behavioral intention concerning system use.

As discussed in Section 2.5.2, TAM is predicated on two powerful and wellresearched constructs that capture behavioral attitudes and that have been repeatedly shown to influence users' intention to use a technology. These are perceived usefulness and perceived ease of use. These constructs were recast in Venkatesh et al. (2003)'s UTAUT model as performance expectancy and effort expectancy respectively. The UTAUT constructs capture the original meaning of the TAM constructs, but have the additional intent and benefit of unifying other closely-related constructs in the literature.

Given the slightly broader definition of the UTAUT constructs and their intent to consolidate the overlapping constructs found in the literature, they 
were selected over the original TAM constructs for this research. However, the UTAUT constructs, due to their similarity to TAM definitions and intent, will be considered interchangeable with their source TAM constructs when considering the application of validated theoretical relationships from the literature. These two technology acceptance constructs have been included in the conceptual research model in order to represent the generic technology acceptance factors associated with the use of an information technology. These two constructs and their associated research propositions will be considered in more detail below.

\subsubsection{Effort Expectancy}

It is expected that an easy to use system will present lower barriers to use: the lower the effort, the more likely a user will be to consider using the system to complete a task. In accordance with the published and validated relationships in the technology acceptance literature, effort expectancy (Venkatesh et al., 2003, p. 450) - see Section 2.5.2 - is expected to positively impact both a user's intention to use a technology and the performance expectancy (Venkatesh et al., 2003, p. 447) of that technology. The associated research propositions are as follows:

- Research Proposition 1 (P1): effort expectancy (perceived ease of use) will have a positive impact on a user's intention to use an electronic recordkeeping system.

- Research Proposition 2 (P2): effort expectancy (perceived ease of use) will have a positive impact on a user's performance expectancy (perceived usefulness) of an electronic recordkeeping system.

\subsubsection{Performance Expectancy}

The second technology acceptance construct, performance expectancy (perceived usefulness) (Venkatesh et al., 2003, p. 447), has been found to be positively and strongly associated with intention to use in every study consulted that attempted to validate technology acceptance constructs in organizational contexts (see Section 2.5.2). It is expected that this trend will continue amongst EDRMS users, with those users who perceive the system to be useful continuing to have a strong intention to continue to use it. The associated research proposition is as follows: 
- Research Proposition 3 (P3): performance expectancy (perceived usefulness) will have a positive impact on a user's intention to use an electronic recordkeeping system.

As illustrated in the conceptual research model (Figure 3.1), given that an electronic recordkeeping system necessarily operates within an organization, a user's willingness to participate in storing records in an electronic recordkeeping system is also anticipated to be influenced by external social factors associated with the user's immediate environment (their organizational context). Therefore, constructs capturing the organizational context of EDRMS use are also expected to influence the intention to use.

\subsection{Organizational Context Factors}

Users of EDRMS are necessarily associated with an organization and are thus under the influence of social pressures and norms that form that organizational context. These social influences represent a continuum of behaviors. Based on the literature, the conceptual research model has adopted two representative constructs: social influence (based on the subjective norm) and perceived power security, which attempt to reflect the primary sources of social influences expected to impact a user's decision to use an EDRMS.

\subsubsection{Social Influence}

As discussed in Section 2.5.2, social influence (Venkatesh et al., 2003, p. 451) is a well-studied construct that has proven its worth through standardized and validated measurements. As with the technology acceptance constructs, social influence is the UTAUT construct that is defined in an attempt to combine closely-related constructs from the literature, and it is based fundamentally on the subjective norm. As with the previous constructs, this research will favor the broader intent and definition of the UTAUT construct; however, social influence and the subjective norm will be used interchangeably when applying findings from the literature concerning the proposed relationships of this construct with others in the model.

Within an organization, employees are likely to be influenced in deciding whether to use an EDRMS both by what their managers may think (e.g., the impact of performance reviews) and what their peers think (e.g., the desire to conform to the social norm), as well as the need to work flexibly in teams 
with other employees who may require or demand access to their records. Furthermore, earlier research has provided evidence that social influence (subjective norm) has a significant effect on intention to use, either directly or indirectly, through its effects on performance expectancy (perceived usefulness) (Karahanna, Straub, \& Chervany, 1999; Venkatesh \& Davis, 2000).

The associated research propositions are as follows:

- Research Proposition 4 (P4): social influence (subjective norm) will have a positive impact on a user's performance expectancy (perceived usefulness) of an electronic recordkeeping system.

- Research Proposition 5 (P5): social influence (subjective norm) will have a positive impact on a user's intention to use an electronic recordkeeping system.

\subsubsection{Perceived Power Security}

As discussed in Section 2.5.2, perceived power security is a second, lessresearched social construct that is defined as "the degree to which a person believes that using a particular system will be free from threats of insecurity regarding loss of power and influence" (Ong et al., 2005, p. 3).

EDRMS technologies have the potential to disrupt both authoritative (extending over people) and allocative (extending over objects or materials) types of power resources (Orlikowski, 1992, p. 405). By increasing the transparency of individuals' contributions to the EDRMS, individuals may feel more open to being judged with an associated loss of security. Also, by centralizing recordkeeping into an EDRMS, individuals with allocative power over legacy systems and/or individual documents may perceive a loss of power to those who administer and manage the EDRMS. As such, users who choose to use the system are expected to experience a shift in power associated with control over their records, and they are likely to resist due to concerns about their power relationships. The more secure a user feels in terms of their power relationships, the more that person is likely to intend to use the system:

- Research Proposition 6 (P6): perceived power security will have a positive impact on a user's intention to use an electronic recordkeeping system.

In addition, the literature indicated that the more insecure or negative employees' expectations are, the more negative are their affective reactions 
including apprehension, anxiety, stress, and fear. Hackbarth et al. (2003) have shown that there is a negative relationship between computer anxiety and users' perception of ease of use. This leads to the next research proposition.

- Research Proposition 7 (P7): perceived power security will have a positive impact on a user's effort expectancy (perceived ease of use) of an electronic recordkeeping system.

The selection of social influence and perceived power security also represent the possible 'interactions' proposed in Giddens' Structuration Theory (p. 20)-communication, power, and sanction. This provides a theoretically grounded basis for the selection of the organizational context constructs, their proposed interrelationships, and ultimately the selection of their underlying measurement items.

\subsection{Knowledge Interpretation}

As discussed in Section 2.5.2, the final determinant of system use focuses on a user's perceptions surrounding the importance and usefulness of the recordkeeping process itself. It focuses on the user's needs and their interpretation of the value of recorded documents: knowledge interpretation.

The location of the individual on that value continuum is expected to impact their willingness to use an EDRMS to store documents and records for use in the future. For the records manager and archivist, the purpose of and need for records is clear: the activity of recordkeeping has value and is a necessary part of a functioning society (Pederson, 2003). However, this view of the value of stored knowledge and records is not necessarily shared by everyone. Nonetheless, due in part to the volume of documents being created in modern society, current EDRMS designs require end users (rather than records managers or records clerks) to make the actual effort to nominate, classify, and store documents and records into the system (Joseph et al., 2012). However, not all end users share the same interpretation of the value of records, and many end users may believe that formal storage of those documents and records is simply not worth the effort, instead relying on personal collections based on their personal anticipated information needs (Bruce, 2005).

Schultze and Stabell (2004) defined four knowledge discourses (Figure 2.16 on p. 52) to illustrate different world views and differing interpretations concerning the value of recorded knowledge. Furthermore, each discourse is 
tuned to work within an organizational context, the same context as shared by current implementations of EDRMS. However, of those four world views, only one of them - that of the neo-functionalists - appears to value the capture of records and the process of recordkeeping in a consensus-oriented organizational context.

The literature suggests that the main difference in the ontological perspectives of these knowledge discourses is the perceived value-duration of a particular knowledge artifact. Those adhering to a neo-functionalist or critical knowledge discourse see all records as potential long-term 'assets' that are worthy of keeping and managing for use in the future. In contrast, those adhering to a constructivist or dialogic knowledge discourse perceive the same knowledge artifacts as merely supporting the current 'working knowledge' (i.e, having only short-term value). These latter individuals would see little or no value in investing in the effort to formally classify and manage documents and/or records beyond their current application.

Lacking a suitably validated construct from the literature, this study further developed the perceived value of records. This was initially defined ${ }^{1}$ as a user's belief that a document ${ }^{2}$ has value beyond the current application and is worthy of storing for the future. Note that this is more targeted than existing qualitatively described constructs including the value accorded to information (Oliver, 2008) or the value of information (Svärd, 2014).

It is expected that individuals who have a higher perceived value of records will believe that an EDRMS is a useful tool to capture and manage those documents and records into the future. The associated research proposition is:

- Research Proposition 8 (P8): perceived value of records will have a positive impact on a user's performance expectancy (perceived usefulness) of an electronic recordkeeping system.

Furthermore, it is expected that individuals with a higher perceived value of records will also see the entire exercise of electronic records management as an activity worthy of their time and effort, consequently increasing their intention to use the system. The final associated research proposition is:

\footnotetext{
${ }^{1}$ For the final refined definition of Perceived Value of Records, see Section 8.2.3, p. 201.

${ }^{2}$ Refer to Section 2.2 and the broader definition of document: any "recorded information or object which can be treated as a unit" (ISO 15489.1, 2002, p. 3).
} 
- Research Proposition 9 (P9): perceived value of records will have a positive impact on a user's intention to use an electronic recordkeeping system.

The resulting research model (see Figure 3.2) illustrates the proposed relationships between the selected constructs. Furthermore, it provides an illustration of the relationship of the construct measurement items (In) to the constructs. It is expected that these constructs will work together to increase or decrease a user's intention to use an EDRMS.

For example, it can be envisioned that even if an individual believes that a particular document has value and would 'normally' be willing to participate in recordkeeping (i.e., has a high perceived value of records), this 'willingness' (intention to use) may be contingent on (or be correlated with) the organizational political context, with lower intention to use observed in dissensus organizational contexts.

Likewise, individuals with a low perceived value of records may perceive efforts to systematize and store this 'low value' knowledge as a form of conspiracy and/or a developing threat against the workers, thus further steeling their intention to not participate.

\subsection{Chapter Summary}

This chapter has presented a conceptual research model derived from the literature. The core of the model is based on the technology acceptance literature, where the constructs effort expectancy and performance expectancy and their proposed interactions are presented. Next, electronic recordkeeping technology is necessarily used in an organizational context, with this conceptual area being represented by two additional constructs, social influence and perceived power security, and their proposed interactions. Finally, the interpretation required to value electronic records and the process of recordkeeping was addressed via individuals' knowledge interpretation, which included the introduction of a new construct: the perceived value of records. The constructs and their associated research propositions were integrated into a single research model. 


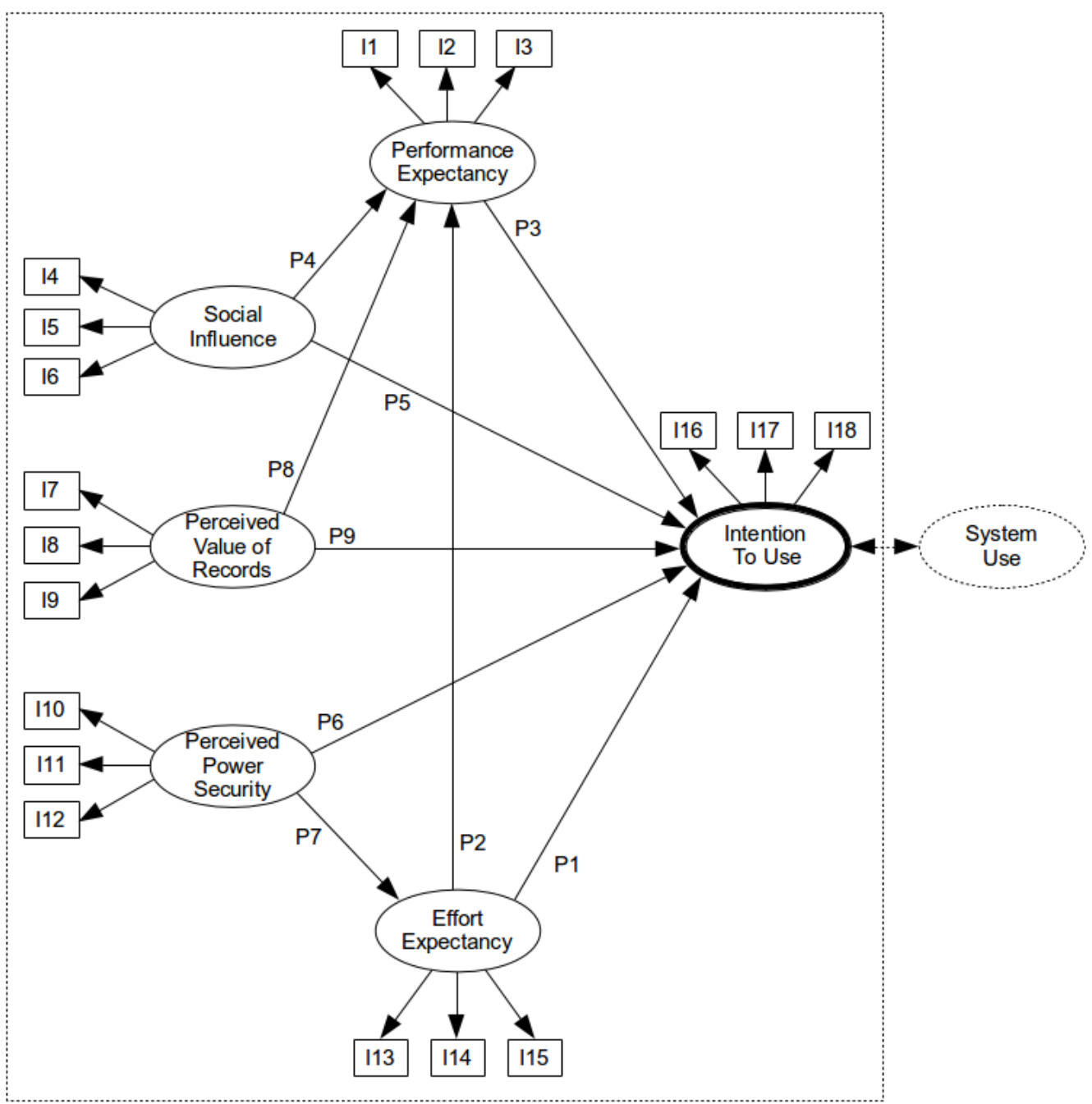

Figure 3.2: Research model with designated research propositions (P1-P9) and example construct measurement item relationships. 


\section{Chapter 4}

\section{Research Design and Methodology}

Contents

4.1 Chapter Overview . . . . . . . . . 67

4.2 Research Paradigm ............. 68

4.3 Sample Population . . . . . . . . . . 70

4.4 Research Methodology ........... 71

4.5 Chapter Summary . . . . . . . . . . 80

\subsection{Chapter Overview}

This chapter explains the design and methodological approach used for the empirical phase of the research. Once the conceptual research model was established and the research propositions developed from a foundation of theory and prior research (see Chapter 3), it was necessary to plan the research itself. This plan would ultimately provide the mechanism for answering the initial research question and addressing each of the research objectives. The first section outlines the philosophical stance used to approach the research and identifies the paradigm that influenced the subsequent approach for each research phase. Next, the sample population is considered, as the approach required to access this group influenced the final selection of research methodology. Finally, the research methodology is discussed with a focus on 
two distinct phases of the research, the first being exploratory and mainly qualitative, the second being confirmatory and quantitative - a means of testing and validating the research model.

\subsection{Research Paradigm}

Prior to embarking on a new piece of research, the researcher is expected to consider any underlying assumptions concerning how and what they will learn during their inquiry. Creswell (2008) summarized the sources of these assumptions and knowledge claims as follows:

Philosophically, researchers make claims about what is knowledge (ontology), how we know it (epistemology), what values go into it (axiology), how we write about it (rhetoric), and the process for studying it (methodology) (Creswell, 2008, p. 6).

Bhaskar (1975) framed three broad ontological positions of knowledge in the philosophy of science, and advocated transcendental realism as, in his opinion, it more closely approximates reality. ${ }^{1}$ Transcendental realism was similarly selected as the base ontology for this research as it regards the objects of knowledge as "the structures and mechanisms that generate phenomena" and regards those objects/structures as real (in the Humian sense); however, it acknowledges that knowledge of those objects is necessarily produced in "the social activity of science" (Bhaskar, 1975, pp. 24-25), thereby adding an interpretive element to this ontological foundation.

This balance between positivist and interpretivist perspectives is found throughout recordkeeping research and theory. As discussed in Section 2.3, recordkeeping theory has been heavily influenced by Giddens' Stucturation Theory (Giddens, 1984; Upward, 1997). Like Bhaskar, Giddens developed Structuration Theory as a way of overcoming what he saw as deficiencies in the two dominant epistemological and methodological approaches used in social analysis in the late 1970s and early 1980s (M. Jones \& Karsten, 2008, p. 130); in other words, he found both positivist/naturalist as well as interpretive sociologies to have significant weaknesses when applied to sociological issues.

\footnotetext{
${ }^{1}$ This is in contrast to classical empiricism, as put forward by Hume, which asserts that the ultimate objects of knowledge are 'atomistic events' and that these, if known, would perfectly constitute given facts and ultimately reality; and transcendental idealism, as formulated by Kant, which asserts that all objects of knowledge are merely idealized models, and knowledge is a "structure rather than a surface" (Bhaskar, 1975, p. 25).
} 
Thus, Structuration Theory sought to "avoid such asymmetrical and dualistic treatment of action and structure by conceptualizing the two as a mutually constitutive duality" (M. Jones \& Karsten, 2008, p. 131).

However, Giddens did not offer a methodological recommendation for those who choose the structurational theoretical perspective. In fact, he admitted: "I do not try to wield a methodological scalpel... there is [nothing] in the logic or the substance of structuration theory which would somehow prohibit the use of some specific research technique, such as survey methods, questionnaires or whatever" (Giddens, 1984 as quoted in M. Jones \& Karsten, 2008 , p. 131). However, those who choose to use quantitative tools must guard against the temptation to claim a complete understanding based on their quantitative results.

That said, the theoretical perspective (paradigm) claimed for this research is post-positivism (Creswell, 2008, p. 7). The relationships of the constructs derived from the literature review - as set forth in the conceptual research model - imply determination, reductionism, empirical observation and measurement, and theory verification (i.e., a positivist/naturalist heritage). The methodology best able to test the relationship between variables is the survey, as it can collect data that is able to test theory and causal relations, and does so from "theoretically grounded expectations about how and why the variables ought to be related" (Pinsonneault \& Kraemer, 1993, p. 9). Nonetheless, as Giddens highlighted, the 'variables' themselves are based on a foundation of interpretive constructivism.

Methodologically, this research thus required a mixed-methods approach (Creswell, 2008). Part of the research activity included the investigation and development of measurement items and the exploration of the research model's new construct (perceived value of records), which had yet to be fully defined and validated quantitatively. This initial phase of the research necessarily included the use of qualitative methods in order to explore and gain an understanding of how people view, understand, and relate to the essence of the model constructs, and how they communicate that meaning. This exploratory work was required in order collect and validate suitable measurement items for use in the quantitative survey and its subsequent statistical analysis. 


\subsection{Sample Population}

The sample was drawn from a population consisting of employees from a New Zealand public sector organization that has implemented an EDRMS. ${ }^{2}$ The unit of analysis is the individual EDRMS user.

New Zealand has taken a strong stance on electronic recordkeeping. The New Zealand Government was an early adopter of ISO 15489 and placed the international standard materially into force after passing the Public Records Act (2005). The Public Records Act sets a mandatory framework for recordkeeping in public offices and local authorities ${ }^{3}$ - i.e., the public sector.

A 2010 Government Recordkeeping Survey of Public Offices (which was the last survey of its type) found that New Zealand Government offices used a variety of recordkeeping systems, with the majority of public offices (93\%) continuing to use paper systems for one or more record series. In terms of electronic records, only $44 \%$ used compliant electronic recordkeeping systems. Furthermore, $28 \%$ of public offices reported that they were undertaking a project to implement a system to manage electronic records (Dudding \& Mayo, 2010b). In addition, the 2010 Government Recordkeeping Survey of Local Authorities found similar trends with $59 \%$ of local authorities reporting use of electronic recordkeeping systems, and 33\% undertaking a project to implement a system to manage electronic records (Dudding \& Mayo, 2010a).

In addition, Archives New Zealand was actively developing a Government Digital Archive, with an associated Digital Continuity Action Plan, which claimed "to be a world first initiative to prevent important public records being lost and ensuring that today's information is available tomorrow" (Archives New Zealand, 2011). This national system of electronic records requires the compliant use of electronic recordkeeping metadata in order to maintain the context (and authenticity and reliability) of the electronic records for the planned transition from the organization's EDRMS to the new national repository.

Of particular interest to this study are ongoing efforts to capture electronic documents and records that are personally authored by employees (e.g., documents associated with projects or policy creation or research). Depending

\footnotetext{
${ }^{2}$ It should be pointed out that the observed EDRMS has been implemented primarily to head office business units, many of whom have used a version of the EDRMS since 2006. However, the organization is also still implementing the EDRMS, as other business units have not yet been transitioned on to the system.

${ }^{3}$ The Public Records Act 2005 does not cover privately owned companies, political parties, or charitable trusts.
} 
on the organization, project or policy documents are often generated for a particular event or purpose, and then left to the author to manage (or not) beyond that usage event. EDRMS will provide these knowledge workers (often for the first time) with a system that is capable of capturing all of their working papers and communications, and by placing them under a management strategy, transform these documents into records of those activities and events.

This type of record is also likely to be low-volume and of an ad hoc nature, which makes it more challenging to fully capture its context. The targeted individuals are most likely to experience higher personal ownership of their documents and thus exhibit variable EDRMS-use intention depending on their interpretation of the value of the documents and the organizational context in which these documents are generated.

It is therefore this subpopulation - the sample frame consisting of the creators of low-volume, high-value documents that have historically been poorly managed - that was the primary target of this study. The sample was identified with the assistance of EDRMS administrators from within the public sector organizations - forming a judgmental sample in terms of the final business units approached.

The target population consisted of knowledge workers and managers ${ }^{4}$ who generate reports and/or documentation, such as policy/strategy development, project management, business analysis, etc. These individuals were expected to have a personal ownership of their documents, which was expected to impact their willingness to participate in organizational recordkeeping activities.

\subsection{Research Methodology}

As discussed in Section 4.2, this research firmly resides in a post-positivist paradigm as it seeks to identify and measure the factors that impact the use of an EDRMS, and it does so through the use of a survey. This approach implies determination, reductionism, empirical observation and measurement,

\footnotetext{
${ }^{4}$ The target population of users can be contrasted with the records and information management (RIM) professionals who manage more rigid and structured records series within the same organizations, such as HR personnel files or case files that have historically been well managed in both paper and electronic series. The professional records clerks are less likely to have emotional connections with the records under management as these structured records were likely generated by someone else and/or created as part of an established business process, have a high volume, and are part of a daily routine. Professional recordkeepers were excluded from the study.
} 
and theory verification (i.e., a positivist/naturalist heritage). Nonetheless, Giddens highlighted that the variables themselves (in this case, the constructs and survey measurement items) are based on a foundation of interpretive constructivism. As such, this research required the use of a mixed-methods approach.

Mixed-methods as it pertains to a research methodology is described as follows:

Mixed-method inquiry intentionally combines different methods that is, methods meant to gather different kinds of information. [...] The underlying premise of mixed-method inquiry is that each paradigm offers a meaningful and legitimate way of knowing and understanding. The underlying rationale for mixed-method inquiry is to understand more fully, to generate deeper and broader insights, to develop important knowledge claims that respect a wider range of interests and perspectives. (Greene \& Caracelli, 1997, p. 7)

When seeking support for the use of mixed-methods, Greene, Caracelli, and Graham (1989) identified five purposes for mixed-method evaluations: triangulation, complementarity, development, initiation, and expansion. Of these five purposes, development is the primary reason for mixing methods in the context of this research project. Greene et al. (1989) state the purpose and rationale for using development as a justification for adopting mixed-methods as follows:

PurPose: Development seeks to use the results from one method to help develop or inform the other method, where development is broadly construed to include sampling and implementation, as well as measurement decisions (Greene et al., 1989, p. 259).

RATiOnALE: [Development seeks to] increase the validity of constructs and inquiry results by capitalizing on inherent method strengths (Greene et al., 1989, p. 259).

The research design thus consisted of two phases that align with the research objectives as outlined in Section 1.4, and that generally follow the methodological processes for construct creation and validation put forward by Moore and Benbasat (1991) and Lewis, Templeton, \& Byrd (2005). The two research phases are as follows: 
1. Phase I: Qualitative Phase consisted of qualitative methods used to develop a pool of potential measurement items as they pertain to the conceptual research model and its constructs.

This phase was used to address aspects of the first research objective: To identify the factors that influence a user's intention to use an electronic recordkeeping system.

2. Phase II: Quantitative Phase consisted of quantitative methods to validate the links between the measurement items and their constructs, and - via the use of a survey - test the research model.

This phase was used to address aspects of the second research objective: To determine the relative importance of the factors that influence a user's intention to use an electronic recordkeeping system.

The qualitative and quantitative phases of the research design are discussed in more detail below.

\subsubsection{Phase I: Qualitative Methods}

Once the conceptual research model's constructs were identified (see Figure 3.2 on p. 65), the next step of the research design was to validate that the proposed constructs worked within the context of electronic recordkeeping and to identify a pool of suitable measurement items.

The four specific objectives of the qualitative research phase were to:

1. validate the proposed constructs and measurement items derived from the literature in the context of electronic recordkeeping;

2. validate the new construct, perceived value of records;

3. identify additional factors that may impact a user's intention to use an electronic recordkeeping system and that are not specifically covered by the constructs of the research model; and

4. validate the relationships of the respective measurement items to their parent constructs.

The research design of the qualitative phase consisted of three stages: Stage 1: in-depth personal interviews to develop an initial pool of measurement items; Stage 2: proofreading with attention to the comprehension of the measurement items; and Stage 3: card sorting to validate the relationships between the constructs and their measurement items. 


\section{Stage 1: Interviews}

As recommended by Suhr (2006), the conceptual research model in Chapter 3 was developed by first reviewing the relevant theory and research literature to support the model's specification. All but one of the research model's constructs have been sourced from the literature and come with pre-validated measurement items. The exception is a newly proposed construct: perceived value of records. Stage 1 explored the meaning and definition of this new construct, generally following the established construct-building procedures as put forward by Churchill (1979) and Lewis et al. (2005), as well as exploring the suitability of the rest of the constructs in the context of electronic recordkeeping.

The technique selected to develop and explore the constructs was the interview (Byrne, 2001b). A semi-structured set of interview questions was administered to a dozen individuals who were selected from the targeted sample population. A convenience/judgmental sample (Boudreau, Gefen, \& Straub, 2001; Pinsonneault \& Kraemer, 1993) was deemed to be suitable for the purposes of this exploration as the targeted constructs were relatively constrained. The interviews were digitally recorded and a written transcript of each of the interviews was created to support the subsequent coding and thematic analysis (Byrne, 2001a; Woods, Priest, \& Roberts, 2002).

The resulting themes were then converted into a pool of measurement item statements. At this point, the measurement items were independent of their research model constructs, as one of the goals of this stage of the research was to provide support for the constructs by first independently creating the foundational pool of measurement items. ${ }^{5}$

\section{Stage 2: Proofreading}

This stage sought to increase the quality of the pool of measurement items. Moore and Benbasat (1991) discussed the need for 'valid item statements' to be developed by the authors. To improve the item statements, ten volunteers were recruited to provide assistance and input. These volunteers were given a limited background concerning the research project, and were simply told that the system referred to is an electronic recordkeeping system. They were

\footnotetext{
${ }^{5}$ It was possible that some emergent measurement items would not be explained by the model's constructs, potentially suggesting the need for additional constructs to fully capture the determinants of EDRMS-use. Subsequent to the analysis, no additional constructs were found to be required.
} 
then asked to review the pool of measurement items and to comment on each item's clarity and comprehensibility.

Individual feedback on the measurement items was obtained and considered in context with other feedback in an effort to maximize the clarity and shared understanding of the essence of each measurement item. Problematic measurement items were reworked to incorporate the feedback. The final wordings were checked for tense and clarity, and only then were the measurement items tentatively associated with a parent construct.

\section{Stage 3: Card Sorting}

Stage 3 focused on scale development (Moore \& Benbasat, 1991; Jackson \& Paunonen, 1985) and construct validation (Hinkle, 2008; Peter, 1981). Construct or content validity is defined as "the degree to which the score or scale being used represents the concept about which generalizations are to be made" (Bohrnstedt, 1970, p. 91 as referenced in Davis, 1989, p. 323).

The technique selected to develop and explore construct validity is card sorting (Spencer \& Warfel, 2004). The goal of this stage is to verify that the pool of measurement items (from Stage 1 and Stage 2) individually load onto one (and only one) of the constructs from the research model. To do this, individual measurement items were written on separate cards, and these cards were then sorted into groups by different participants.

There are two main variations of card sorting that were used to measure different aspects of construct validity. Closed card sorting (Spencer \& Warfel, 2004 ) is where categories are provided to the participants, and they are then asked to sort each measurement item against the available categories. Open card sorting (Faiks \& Hyland, 2000) is where participants are asked to create their own groupings. These two techniques measure two different aspects of validity (Rugg \& McGeorge, 2005; Boudreau et al., 2001), with the former measuring convergent validity - the likelihood that different judges agree that an item reflects the same construct; and the latter measuring discriminant validity - the likelihood that an item unambiguously reflects a single construct (and only that construct).

The goal of this qualitative stage was to maximize the shared understanding of the relationship between a measurement item and its construct, while acknowledging that the interpretation of the author may not be the same as that of participants. Based on the participant feedback, some measurement items were adjusted to more clearly reflect a particular construct, or they were allocated to a different construct, or - in the case of vague measure- 
ment items that appeared to link to multiple constructs - some measurement items were removed from the pool. This also provided the opportunity to determine if the set of constructs in the research model were both necessary and sufficient to explain the set of measurement items that emerged from the Stage 1: interviews.

The outcome of the Stage 3: card sorting was a pool of measurement items with a strong qualitative link to a parent construct. At this point in the research, the research design switched to a primarily quantitative methodological phase.

\subsubsection{Phase II: Quantitative Methods}

Phase II of the research design focused on quantitative methods - specifically on the development and delivery of a survey instrument that was capable of gathering sufficient data to quantitatively validate the relationship between the measurement items and the constructs, and ultimately determine the relative importance of the factors in the research model.

Building on Stages 1-3 of the qualitative phase, the research design of the quantitative phase consisted of three additional stages: Stage 4: Survey Design covered the design of the survey instrument and its implementation within a suitable online survey technology; Stage 5: Survey Pretest included running a pretest of the online survey and using the feedback to further refine the survey instrument; and Stage 6: Survey consisted of using the instrument to gather data to validate the underlying research model.

\section{Stage 4: Survey Design}

The survey design stage consisted of transforming the final measurement item pools into a survey instrument. Although this is not specifically a data-gathering stage (although different designs were trialled and discussed), the effort and consideration required to create the survey instrument is considerable, and therefore it warrants its own stage in the research design.

The objectives of Stage 4 included:

1. the selection of an appropriate online survey delivery technology and training in its use;

2. consideration of the data analysis approach and the implications of how and in what detail the data is required to be captured; 
3. the creation of the survey instrument including: design, item grouping, section flow, branding, etc.; and

4. the creation of associated documentation and communications, including: participant information sheets, participation waivers, introductory emails, graduated automated reminder emails, 'thank you' emails, etc.

The outcome of this stage was a draft 'survey instrument package' that included all materials required to initiate the final data-gathering activity. However, awareness of the data analysis approach was also required in order to inform how the data would be captured by the survey instrument.

The selected analysis approach is one which focuses on confirmatory factor analysis (CFA) as opposed to exploratory factor analysis (EFA) (Suhr, 2006). A class of structural equation modeling was selected as the analysis method because it provides "inferential data analysis and hypothesis testing where the pattern of inter-relationships among the study constructs are specified a priori and grounded in established theory" (Hoe, 2008, p. 76). Specifically, Partial Least Squares Path Modeling (PLS-PM) was selected, because

Unlike the classical covariance-based approach, PLS-PM does not aim at reproducing the sample covariance matrix. PLS-PM is considered as a soft modeling approach where no strong assumptions (with respect to the distributions, the sample size and the measurement scale) are required. (Vinzi, Trinchera, \& Amato, 2010, p. 48)

The PLS-PM analysis method is discussed in greater detail in Section 7.4. However, its identification at this point helped to determine the level of detail and the format required for the survey data collection.

A five-point Likert scale was selected and used for the design of the survey instrument, as it has been found to be sufficient in capturing a measure of reliability (Hinkin, 1998). In addition, design decisions, such as making questions mandatory and ordering them in terms of difficulty, were taken during the design stage (and during the analysis and interpretation stages) in order to avoid common pitfalls associated with statistical data analysis (Helberg, 1995).

The next stage sought to increase the quality of the survey instrument and refine the orchestration of the survey activities through a pretest of the entire survey instrument package. 


\section{Stage 5: Survey Pretest}

The pretest stage focused on two aspects of the online survey: the testing of the survey instrument itself; and the familiarization with and testing of the survey distribution platform and mode of transmission and data collection.

Although a pretest is generally recommended, Dillman (2007) warns that the survey pretest is often done "haphazardly, if at all." He further emphasizes that a properly conceived pretest phase is a chance to:

- evaluate the distribution procedures and see whether any problems arise;

- discover production mistakes by having a few people fill out the survey;

- learn whether people clearly understand all of the questions; and

- provide an opportunity for feedback to improve the survey.

In the case of this online survey, it was also where the technology meets the measurement items, and it provided the researcher with an opportunity to pretest the survey instrument as well as the platform on which it would be administered.

The research design for the survey pretest consisted of administering the survey to 10 participants selected as a convenience sample. These individuals were debriefed subsequent to the survey in an attempt to discover any production mistakes or distribution errors, and they were also given the opportunity to provide additional feedback to improve the survey. The outcome of this stage was the final draft of the survey instrument.

\section{Stage 6: Survey}

The main data-gathering stage in the research design was the final survey. The survey was distributed to a sample selected from the target population (see Section 4.3) and it utilized a communication plan based on the modified Tailored Design Method (Dillman, 2009), the intention of this method being to minimize non-response bias (Sivo, Saunders, Chang, \& Jiang, 2006).

Sample Size: The required sample size was estimated based on the number of constructs required to provide sufficient explanatory power for the research model. For structural equation modeling in general, this is set forth as a 'formula', with a range of 5-20 participants required per parameter estimated 
(Suhr, 2006). The actual number of participants per parameter required is affected by the normality of the data and the estimation method used; however, for PLS-PM, 10 participants per construct is the generally agreed number (Schreiber, Nora, Stage, Barlow, \& King, 2006). Furthermore, a 'critical sample size' or rule-of-thumb sample of 200 is also mentioned elsewhere in the literature (Garver \& Mentzer, 1999; Hoe, 2008; Hox \& Bechger, 1998). Given that there are 6 constructs in the conceptual research model, a multiplier of 10-20 (resulting in 60-120 participants) would provide the lower required sample size estimate. For added security and statistical sensitivity, an effort was made to increase the sample size to $\approx 200$ participants. In the end, 193 usable responses were obtained (see Section 7.2, p. 138).

Common Method Variance (CMV): Also known as common method bias, CMV is "variance that is attributable to the measurement method rather than to the constructs the measures represent" (Podsakoff et al., 2003, p. 879). Data gathered from surveys and questionnaires can sometimes be at risk of CMV. This bias can arise from many sources, including the use of a common rater, the manner in which items are presented to participants, the context in which the items are presented, and other external contextual influences that may be independent of the survey instrument (e.g., time, location and media) yet still affect how people respond (S.-J. Chang, van Witteloostuijn, \& Eden, 2010).

In an attempt to control for CMV risk, Chang et al. (2010, p. 181) recommend a number of ex ante and ex post remedies - "use multiple remedies, not just one remedy, in order to assuage the various concerns about CMV." Several of their recommended "remedies" were considered and incorporated into the research design.

- Collect data from multiple sources. This research incorporated data from multiple public sector organizations and used multiple data collection techniques including interviews, card-sorting, and surveys (see Research Stages above) in an effort to minimize source and method bias.

- Design the data collection instruments with care. The survey measurement items were sourced from the literature and cross-referenced with the thematic analysis from the interviews and cardsorted prior to incorporation into the survey instrument in an effort to minimize bias. Subsequent to data collection, the measurement items also underwent statistical validity checking. 
In addition, other recommended CMV-reducing techniques were used when collecting the survey data including: 1) assuring anonymity and confidentiality, 2) neutral phrasing of measurement items, and 3) positive and negative measurement items, and 4) care to make the measurement items clear and unambiguous. These steps removed many of the potential biases from the comprehension stage of the response process.

- Complicated regression models reduce the likelihood of CMV. Response bias is more likely to emerge in simple survey models, so "complicated specifications of regression models reduce the likelihood of CMV" (S.-J. Chang et al., 2010, p. 179). The analysis of the survey data required the use of partial least squares path modeling paired with statistical validity checks, thereby removing an additional source of potential CMV risk.

These methods, combined with an emphasis on keeping the response rate as high as possible to avoid non-response bias (Sivo et al., 2006; Dillon, Madden, \& Firtle, 1990), were put in place to manage CMV risk.

\subsection{Chapter Summary}

This chapter has explained the approach to the research design and methodology used in the empirical phase of the research. The first section outlined the philosophical stance behind the research, and illustrated the need for different methods to approach each of the research phases. Next, the sample population was described in more detail, as the approach required to access this group would dictate aspects of the final research design. Finally, the research methodology was discussed with a focus on the two distinct phases of the research, the first being qualitative and exploratory, and the second being quantitative and confirmatory - a means of testing and validating the research model.

In the next two chapters, the results of Phase I: Qualitative Methods (Stages 1-3) and Phase II: Quantitative Methods (Stages 4-6) are discussed in more detail. 


\section{Chapter 5}

\section{Phase I: Qualitative Methods}

\section{Contents}

5.1 Chapter Overview . . . . . . . . . . 81

5.2 Stage 1: Interviews ............ 82

5.3 Stage 2: Proofreading . . . . . . . 107

5.4 Stage 3: Card Sorting . . . . . . . . . 112

5.5 Chapter Summary . . . . . . . . . 126

\subsection{Chapter Overview}

As noted in Chapter 4: Research Design 8 Methodology, this chapter will cover Phase I of the empirical research in more depth. Phase I relied on qualitative methods to develop a pool of potential measurement items and related them to the conceptual research model and its constructs. The purpose of Phase I was to address the first research objective: to identify the factors that influence a user's intention to use an electronic recordkeeping system.

The research covered in this chapter was conducted in three stages of data gathering and analysis, where each stage built on the results of the previous stage. The stages were as follows:

1. Stage 1: Interviews - interviews were conducted and then converted, through thematic analysis, into a pool of measurement items. 
2. Stage 2: Proofreading - external input was sought to create clear measurement items that maximized the shared understanding of each statement.

3. Stage 3: Card sorting - relationships were created between measurement items and their parent construct.

In the next three sections, each of the above stages are discussed in detail, including the methods used to capture the data, the results that were obtained, and the analysis and implications that were considered before moving on to the next stage. The Phase I research activities covered in this chapter set the stage for the Phase II quantitative methods that are covered in Chapter 6 .

\subsection{Stage 1: Interviews}

The first stage of the qualitative research phase began with interviews. The specific objectives of this stage were to:

1. validate the existing set of construct measurement items derived from the literature in the context of electronic recordkeeping and further exploring potential determinants of each construct;

2. explore the new construct: perceived value of records; and

3. search for additional items or factors that may impact a user's intention to use an electronic recordkeeping system and that are not specifically covered by the constructs of the research model.

\subsubsection{Interview Instrument Creation}

In consideration of Stage 1's research objectives (stated above), a questionnaire was created that presented the questions without disguising the purpose of the interview (Smith \& Albaum, 2012). However, in order to explore the answers and follow-up on interesting comments that might provide evidence of unanticipated research factors, the structured questionnaire was only used as a guide, incorporating unstructured and probing, open-ended questions as deemed appropriate and necessary by the interviewer.

The resulting interview instrument (see Appendix A) is roughly divided into four sections. The first section starts with simple questions concerning the individual, their role in the organization, and their experience with the 
recordkeeping system technology. The second section explores the various constructs suggested by the literature review as they appear in the conceptual research model. The third section then focuses on the new construct perceived value of records - approaching the issue from several directions. The final section explores the participant's intention to continue to use the technology in the future and provides the opportunity to add additional ideas or anecdotes as they see fit.

\subsubsection{Data Collection Methods}

The dyadic nature of interviews is one in which bias and error are likely to be present, which may be a result of interviewer and interviewee background characteristics, psychological attributes, and behavior (Smith \& Albaum, 2010). In order for the interview instrument to successfully capture a useful amount of high-value information, a wider range of factors than 'good questions' must be taken into account. Some of these include: context, content, structure, disclosure, feedback, and levels of cooperation and/or conflict (Stewart \& Cash, 2008).

In addition, Smith and Albaum (2012, p. 41) emphasize that dyadic interactions can be affected by other intervening factors that can be controlled by the interviewer:

The interviewer can likewise control the style of interviewing and thereby affect the quality of information obtained. For example, styles can range from socio-emotional (maintaining a warm, sympathetic, and understanding relationship with the respondent) to formal (where the person-oriented actions of the interviewer are held to a socially acceptable minimum). The researcher must then assess the desirability of one style over the other in a given situation.

With the above firmly in mind, twelve (12) interview participants were sought from the New Zealand public sector who also met the criteria of the target population (see Section 4.3). The sample consisted of a judgment sample $^{1}$ of four individuals selected from each of three different organizations, representing a variety of roles and management levels. All were willing to

\footnotetext{
${ }^{1}$ The interview participants were recommended by the organizations' records managers based on a verbal description of the targeted sample population. They were generally 'good users' of their systems and had been involved with attempts to recruit others to use the system.
} 
discuss the use of their electronic recordkeeping system within the context of their organization.

In order to promote trust and cooperation, the interviews were held in quiet meeting rooms or private offices that were familiar to the interviewee. A positive socio-emotional approach dominated the tone of the interviews, with friendly non-verbal queues and feedback used liberally to encourage disclosure. Furthermore, the structure of the interview instrument was designed so that the more difficult questions were posed in the second half of the interview in order to maximize the quality of the interview responses (Byrne, 2001b).

Each interview was approximately one hour in duration, was digitally recorded, and was transcribed by the interviewer immediately after the interview, within a timeframe ranging from 'that afternoon' to 1-3 days after the interview. Copies of the interview transcripts were made available to participants on request. The data contained in the transcripts were used as the basis for the next step - the development of the initial pool of measurement items.

\subsubsection{Thematic Analysis Methods}

The next step in the interview stage focused on the analysis of the interview transcripts in an attempt to derive some meaning and/or patterns that could contribute to the development of a pool of measurement items. Thematic analysis was selected as the most suitable technique (Byrne, 2001a; Priest, Roberts, \& Woods, 2002; Woods et al., 2002). Thematic analysis is a method for identifying, analyzing, and reporting patterns (or themes) within data (Braun \& Clarke, 2006); or in other words, "a form of pattern recognition within the data, where emerging themes become the categories for analysis" (Fereday \& Muir-Cochrane, 2008).

Braun and Clarke (2006, p. 78) argue that qualitative methods fall into basically two camps. In the first camp are found methods that are "tied to, or stemming from, a particular theoretical or epistemological position. [...] Second, there are methods that are essentially independent of theory and epistemology, and can be applied across a range of theoretical and epistemological approaches." They conclude that thematic analysis is "firmly in the second camp." The different steps or phases of thematic analysis as described by Braun and Clarke (2006, p. 87) and are reproduced in detail in Table 5.1.

After consideration of the pros and cons of using software to ease the process of thematic coding for qualitative research (A. Atherton \& Elsmore, 


\section{Phase}

1. Familiarising yourself with your data:

2. Generating initial codes:

3. Searching for themes:

4. Reviewing themes:

5. Defining \& naming themes:

6. Producing the report:

\section{Description of the process}

Transcribing data (if necessary), reading and rereading the data, noting down initial ideas.

Coding interesting features of the data in a systematic fashion across the entire data set, collating data relevant to each code.

Collating codes into potential themes, gathering all data relevant to each potential theme.

Checking if the themes work in relation to the coded extracts (Level 1) and the entire data set (Level 2), generating a thematic map of the analysis.

Ongoing analysis to refine the specifics of each theme, and the overall story the analysis tells; generating clear definitions and names for each theme.

The final opportunity for analysis. Selection of vivid, compelling extract examples, final analysis of selected extracts, relating back of the analysis to the research question and literature, producing a scholarly report of the analysis.

Table 5.1: Thematic Analysis: phases and the description of the thematic analysis process (reproduced from Braun \& Clarke, 2006, p. 87) 
2007), it was decided that the pragmatic goals of this research - namely, the creation of a pool of measurement items - outweighed the difficulties of 'contextual reassembly' that might warrant the use of manual qualitative analysis methods. ${ }^{2}$ To this end, QSR International's NVivo 10 qualitative data analysis software greatly assisted in the completion of the subsequent thematic analysis.

Using NVivo, every phrase and paragraph of the interview transcripts was separately considered and coded. Each theme (or 'node' in NVivo) was formed based on the interpretation of the general intent of an interview statement and was both labeled and defined at creation. Several iterations were necessary to code interview phrases logically and consistently. To assist with coding consistency, a thematic analysis 'dictionary' was created for personal use. This dictionary grew to contain over sixty codes, their working definitions, and 1-3 illustrative quotations per defined code. The number of codes was eventually reduced/combined to form forty (40) themes that effectively emerged from the interview stage.

Two main measures were used to determine the relative importance of a particular theme: the number of participants who mentioned the theme, and the total number of times it came up in the interviews. For example, the importance of 'effective search' in an EDRMS was a theme mentioned by all 12 participants and referred to 51 times. In contrast, the influence of organizational policy on use was only mentioned by 3 participants and on only 4 occasions, while the need for a more flexible EDRMS interface was only mentioned once.

\subsubsection{Item Creation Analysis and Results}

As recommended in Table 5.1, the emergent codes were first developed and applied as a single pool across the entire data set. However, these were later grouped into themes and then further grouped according to the constructs in alignment with the research model (Figure 3.2). Given the challenge of reporting this iterative and interconnected analysis process in a linear fashion, the following sections will detail the development of individual measurement

\footnotetext{
${ }^{2}$ It could be argued that contextual reassembly is more targeted at the 'first camp' of analysis techniques, where methods such as Grounded Theory require the reassembly of themes to create new theory (Corbin \& Strauss, 2008).
} 
items as they appeared by construct. ${ }^{3}$ Each of the construct categories are separately detailed below.

\section{Performance Expectancy (PE)}

To develop and refine the measurement items (and validate the ones taken from the literature), the interviews were first coded with context-specific codes using NVivo. The intent was to capture themes emerging from the interviews themselves that further highlighted electronic recordkeeping-specific issues and requirements (i.e., potential determinants of use). Table 5.2 lists the thematic codes that were tentatively associated with performance expectancy. Examples of the thematic coding include electronic recordkeeping-specific performance criteria such as effectiveness of security control and effectiveness of document classification.

\begin{tabular}{lcc} 
Interview Codes: Performance Expectancy & Sources & References \\
\hline Effectiveness of search & 12 & 51 \\
Personal job productivity & 10 & 27 \\
Safety of storage & 10 & 18 \\
Effectiveness of security control & 9 & 37 \\
Effectiveness of document classification & 8 & 24 \\
Reliability of system (up-time) & 8 & 16 \\
Integration into business process & 5 & 13 \\
Overcoming an existing barrier & 4 & 10 \\
Effectiveness of document management controls & 4 & 15 \\
Speed of system & 4 & 6 \\
Effectiveness of reporting on record status & 3 & 8 \\
\hline TOTAL & 12 & 225
\end{tabular}

Table 5.2: Performance Expectancy: frequency of NVivo node-coding by number of interview participants (sources) and total number of reference quotations (references).

Table 5.2 details all of the final codes/themes that were tentatively grouped against performance expectancy. In order to fully capture the shared meaning

\footnotetext{
${ }^{3}$ The constructs representing the independent variables of the conceptual research model are the focus of this stage. Measurement items for the dependent variable were dealt with during the creation of the survey instrument.
} 
and shared understanding of how participants define usefulness (performance expectancy) in the context of electronic recordkeeping system, this stage of the research sought to combine (and validate) any published measurement items with new measurement items that emerged from the interview stage and the thematic analysis. This superset of performance expectancy measurement items was expected to provide richer and context-specific descriptive power when used to reflect the latent construct during the later quantitative phase (Phase II).

The published measurement items for UTAUT's performance expectancy are reproduced in Table 5.3. A code was assigned to each construct, consisting of the construct abbreviation and a two-digit reference number - e.g., PE03. As the UTAUT measurement items were specifically developed to encompass a very broad definition of 'technology' and 'system', they required minor changes to wording in order to align them with the emergent measurement items or to help better target the context of electronic recordkeeping systems in use in the public sector.

Performance Expectancy: Existing Validated Items \& Source Construct

\begin{tabular}{|l|l|l|}
\hline $\begin{array}{l}\text { Item } \\
\text { Code }\end{array}$ & Measurement Item & Source Construct \\
\hline PE01 & $\begin{array}{l}\text { I would find the system } \\
\text { useful in my job. }\end{array}$ & $\begin{array}{l}\text { Perceived Usefulness (Davis, 1989; } \\
\text { Davis, Bagozzi, \& Warshaw, 1989; } \\
\text { Venkatesh \& Davis, 2000) }\end{array}$ \\
\hline PE02 & $\begin{array}{l}\text { Using the system enables } \\
\text { me to accomplish tasks } \\
\text { more quickly. }\end{array}$ & $\begin{array}{l}\text { Relative Advantage (Moore \& } \\
\text { Benbasat, 1991) }\end{array}$ \\
\hline PE03 & $\begin{array}{l}\text { Using the system } \\
\text { increases my productivity. }\end{array}$ & $\begin{array}{l}\text { Relative Advantage (Moore \& } \\
\text { Benbasat, 1991) }\end{array}$ \\
\hline PE04 & $\begin{array}{l}\text { If I use the system, I will } \\
\text { increase my chances of } \\
\text { getting a raise. }\end{array}$ & $\begin{array}{l}\text { Outcome Expectations (Compeau \& } \\
\text { Higgins, 1995; Compeau, Higgins, \& } \\
\text { Huff, 1999) }\end{array}$ \\
\hline
\end{tabular}

Table 5.3: Performance Expectancy: validated measurement items from the literature (Venkatesh, Morris, Davis, \& Davis, 2003)

The majority of the measurement items in Table 5.3 appeared to be logical and work well in an electronic recordkeeping context. However, based on the interviews, a cultural phenomenon, which can be described as a public sector 
ethos, was evident in the responses. In this context, public sector ethos is defined as the extent to which an individual shares an altruistic philosophy that allows them to separate responsibility from ownership.

This was most in evidence when discussing situations where personal effort would have been expected to lead to personal gain - a key driver in performance expectancy. In these cases, several participants had no expectation of receiving personal recognition. Two examples of public sector ethos from the interview transcripts are as follows:

It's not my work! It's not my document! I'm responsible for doing a good job and creating it, so I'm accountable for creating a good product, but the product isn't. . I don't own it! I can't say: 'I wrote the policy. This is my policy.' Or, 'This is my piece of research.' It is the public's. It is the Government's. It is the Minister's. It's my boss's.

Another participant stated:

I am personally accountable for the quality of the document and the quality of what I produce. But, I can't claim it as... mine - if something goes really well, for example.

Given the lack of apparent connection between effort and reward (specifically 'pay'), the measurement item PE04: "If I use the system, I will increase my chances of getting a raise" was less reflective of the desires or expectations of public sector employees than other potential outcome expectations such as 'recognition.' As such, Item PE4 was updated to: "If I use the system, I will increase my chances of getting recognition in the workplace."

The remaining themes - those not already covered by published measurement items - were then converted into draft measurement item statements and added to the measurement item pool. The final superset of potential measurement items combined the existing (adjusted) UTAUT measurement items with the new draft measurement items that emerged from the thematic analysis. The superset of measurement items relating to the performance expectancy construct category are listed in Table 5.4. 
Performance Expectancy (PE): Measurement Items \& Source Construct

\begin{tabular}{|c|c|c|}
\hline $\begin{array}{l}\text { Item } \\
\text { Code }\end{array}$ & Proposed Measurement Item & Source \\
\hline PE01 & I would find the system useful in my job. & UTAUT (PE) \\
\hline PE02 & $\begin{array}{l}\text { Using the system enables me to accomplish tasks } \\
\text { more quickly. }\end{array}$ & UTAUT (PE) \\
\hline PE03 & Using the system increases my productivity. & UTAUT (PE) \\
\hline PE04 & $\begin{array}{l}\text { If I use the system, I will increase my chances of } \\
\text { getting recognition in the workplace. [adjusted] }\end{array}$ & UTAUT (PE) \\
\hline PE05 & $\begin{array}{l}\text { Using Search allows me to locate important } \\
\text { records effectively. }\end{array}$ & proposed \\
\hline PE06 & $\begin{array}{l}\text { When I store a record in the system, I am } \\
\text { confident it will be there in the future. }\end{array}$ & proposed \\
\hline PE07 & $\begin{array}{l}\text { The system can effectively manage the security } \\
\text { required for our sensitive records. }\end{array}$ & proposed \\
\hline PE08 & $\begin{array}{l}\text { I find the document classification in the system to } \\
\text { be intuitive. }\end{array}$ & proposed \\
\hline PE09 & $\begin{array}{l}\text { From my own experience, I have found the system } \\
\text { to be reliable. }\end{array}$ & proposed \\
\hline PE10 & $\begin{array}{l}\text { I know when I am supposed to use the electronic } \\
\text { recordkeeping system in my job. }\end{array}$ & proposed \\
\hline PE11 & $\begin{array}{l}\text { We use the electronic recordkeeping system as a } \\
\text { way to achieve one or more of our business goals. }\end{array}$ & proposed \\
\hline PE12 & $\begin{array}{l}\text { The integration of the electronic recordkeeping } \\
\text { system with my other office programmes and } \\
\text { tools saves me time. }\end{array}$ & proposed \\
\hline PE13 & $\begin{array}{l}\text { I have noticed that the system is often slow in its } \\
\text { operation. }\end{array}$ & proposed \\
\hline PE14 & $\begin{array}{l}\text { I rely on reports generated from the system as } \\
\text { one of the tools to manage and achieve my job } \\
\text { responsibilities. }\end{array}$ & proposed \\
\hline
\end{tabular}

Table 5.4: Performance Expectancy: proposed set of Stage 1 measurement items combining those sourced from the literature (UTAUT: Venkatesh, Morris, Davis, \& Davis, 2003) with those that emerged from Stage 1's thematic analysis. 


\section{Effort Expectancy (EE)}

Measurement items were then developed for ease of use (effort expectancy) in a similar manner to those developed for performance expectancy. A set of codes/themes as captured in NVivo 10 were categorized against effort expectancy. Table 5.5 provides the emergent codes and themes, including electronic recordkeeping-specific performance criteria such as ease of filing and ease of search.

\begin{tabular}{lcc} 
Interview Codes: Effort Expectancy & Sources & References \\
\hline Clear and understandable interface & 12 & 39 \\
Familiarity through regular use & 10 & 14 \\
Ease of learning & 10 & 30 \\
Ease of filing & 7 & 13 \\
Ease of search & 7 & 13 \\
Ease of records administration & 5 & 7 \\
Convenience - well-integrated & 3 & 5 \\
Flexibility of the system interface & 1 & 3 \\
\hline TOTAL & 12 & 124
\end{tabular}

Table 5.5: Effort Expectancy: frequency of NVivo node-coding by number of interview participants (sources) and total number of reference quotations (references).

The published and validated measurement items for effort expectancy are listed in Table 5.6. These measurement items were designed to encompass a very broad definition of 'technology' and 'system use' and similarly required additional measurement items to fully reflect the latent effort expectancy construct in the narrower context of electronic recordkeeping systems.

A review of the published measurement items showed a very good overlap between the codes/themes that emerged from the interviews and those reflected in the UTAUT effort expectancy measurement items. For example, the most prevalent category of comments from the interviews concerned the need for a clear and understandable interface, with over 39 references representing all 12 participants. Opinions on what that entails were often at odds, for example one participant stated:

As far as the interface is concerned... it's pretty intuitive and easy to use. 
While another participant complained:

I would say that the system is not user-friendly, it is difficult to navigate, it requires a lot of effort to input documents... Yeah, I think it's functionally difficult to use.

Effort Expectancy (EE): Existing Validated Items \& Source Construct

\begin{tabular}{|c|c|c|}
\hline $\begin{array}{l}\text { Item } \\
\text { Code }\end{array}$ & Measurement Item & Source Construct \\
\hline EE01 & $\begin{array}{l}\text { My interaction with the system } \\
\text { would be clear and } \\
\text { understandable. }\end{array}$ & $\begin{array}{l}\text { Perceived Ease of Use (Davis, } \\
\text { 1989; Davis, Bagozzi, \& } \\
\text { Warshaw, 1989) }\end{array}$ \\
\hline EE02 & $\begin{array}{l}\text { It would be easy for me to } \\
\text { become skillful at using the } \\
\text { system. }\end{array}$ & $\begin{array}{l}\text { Perceived Ease of Use (Davis, } \\
\text { 1989; Davis, Bagozzi, \& } \\
\text { Warshaw, 1989) }\end{array}$ \\
\hline EE03 & $\begin{array}{l}\text { I would find the system easy to } \\
\text { use. }\end{array}$ & $\begin{array}{l}\text { Perceived Ease of Use (Davis, } \\
\text { 1989; Davis, Bagozzi, \& } \\
\text { Warshaw, 1989) }\end{array}$ \\
\hline EE04 & $\begin{array}{l}\text { Learning to operate the system } \\
\text { is easy for me. }\end{array}$ & $\begin{array}{l}\text { Ease of Use (Moore \& Benbasat, } \\
\text { 1991) }\end{array}$ \\
\hline
\end{tabular}

Table 5.6: Effort Expectancy: validated measurement items from the literature (Venkatesh, Morris, Davis, \& Davis, 2003)

UTAUT's EE01: "My interaction with the system would be clear and understandable" covers the above mentioned emergent interview theme sufficiently. Similarly, emergent coding associated with 'learning' or 'skill' are also sufficiently covered by existing UTAUT measurement items.

The remaining emergent themes - those not covered by published measurement items - were then converted into draft measurement items and added to the measurement item pool. The proposed superset of effort expectancy measurement items are listed in Table 5.7. 
Effort Expectancy (EE): Measurement Items \& Source Construct

\begin{tabular}{|l|l|l|}
\hline $\begin{array}{l}\text { Item } \\
\text { Code }\end{array}$ & Proposed Measurement Item & Source \\
\hline EE01 & $\begin{array}{l}\text { My interaction with the system would be clear } \\
\text { and understandable. }\end{array}$ & UTAUT (EE) \\
\hline EE02 & $\begin{array}{l}\text { It would be easy for me to become skillful at } \\
\text { using the system. }\end{array}$ & UTAUT (EE) \\
\hline EE03 & I would find the system easy to use. & UTAUT (EE) \\
\hline EE04 & Learning to operate the system is easy for me. & UTAUT (EE) \\
\hline EE05 & $\begin{array}{l}\text { I use the system often enough to maintain my } \\
\text { skill with its use }\end{array}$ & proposed \\
\hline EE06 & $\begin{array}{l}\text { The process of filing - contributing a record - is } \\
\text { easy and straightforward. }\end{array}$ & proposed \\
\hline EE07 & $\begin{array}{l}\text { I have no trouble searching for records in the } \\
\text { system. }\end{array}$ & proposed \\
\hline EE08 & $\begin{array}{l}\text { I can learn how to do administrative tasks } \\
\text { without difficulty. }\end{array}$ & $\begin{array}{l}\text { proposed } \\
\text { of my computer's office tools and programs. }\end{array}$ \\
\hline EE09 & $\begin{array}{l}\text { The system is flexible enough to support my } \\
\text { needs as well as the organization's requirements. }\end{array}$ & proposed \\
\hline EE10 & \\
\hline
\end{tabular}

Table 5.7: Effort expectancy: proposed set of Stage 1 measurement items combining those sourced from the literature (UTAUT: Venkatesh, Morris, Davis, \& Davis, 2003) with those that emerged from Stage 1's thematic analysis. 


\section{Social Influence}

The third major construct in the conceptual research model is social influence, which is based primarily on the subjective norm. This is part of the Organizational Context group of constructs in the conceptual research model and represents the external social influences experienced by users.

Once again, some codes/themes were categorized against the social influence construct. Examples of these codes/themes are provided below in Table 5.8.

\begin{tabular}{lcc} 
Interview Codes: Social Influence & Sources & References \\
\hline Influence of manager's opinion & 11 & 29 \\
Influence of peer's opinion & 11 & 27 \\
Importance of alignment of opinion with others & 10 & 17 \\
Influence of social 'critical mass' in usage & 9 & 18 \\
Status of diligent users & 7 & 12 \\
Importance of manager champions & 6 & 17 \\
Importance of influencing others (being a champion) & 3 & 4 \\
Influence of organization policy (i.e., in code of ethics) & 3 & 4 \\
\hline TOTAL & 12 & 128
\end{tabular}

Table 5.8: Social Influence: frequency of NVivo node-coding by number of interview participants (sources) and total number of reference quotations (references).

The published measurement items used in UTAUT for social influence are listed in Table 5.9. These UTAUT measurement items were specifically designed to encompass a very broad range of likely sources of social influence within a generic organization. Although it was expected that these would be sufficient to cover the majority of the relevant organizational contexts, they were nonetheless validated against the emergent interview themes in the context of electronic recordkeeping in the public sector.

There is excellent overlap between the codes that emerged from the interviews and those reflected in the UTAUT's social influence measurement items. For example, evidence of social influence from the various social strata is well represented, including peers, managers, and senior managers. However, aspects of Image (Moore \& Benbasat, 1991), which did not exhibit strong loading in the UTAUT model, were in evidence as an emergent interview theme. 
Social Influence (SI): Existing Validated Items and Source Construct

\begin{tabular}{|c|l|l|}
\hline Item & Measurement Item & Source Construct \\
\hline SI01 & $\begin{array}{l}\text { People who influence } \\
\text { my behavior think that } \\
\text { I should use the } \\
\text { system. }\end{array}$ & $\begin{array}{l}\text { Subjective Norm (Fishbein \& Ajzen, } \\
\text { 1975; Davis, Bagozzi, \& Warshaw, 1989; } \\
\text { Ajzen, 1991; Mathieson, 1991; Taylor \& } \\
\text { Todd, 1995a, 1995b) }\end{array}$ \\
\hline SI02 & $\begin{array}{l}\text { People who are } \\
\text { important to me think } \\
\text { that I should use the } \\
\text { system. }\end{array}$ & $\begin{array}{l}\text { Subjective Norm (Fishbein \& Ajzen, } \\
\text { 1975; Davis, Bagozzi, \& Warshaw, 1989; } \\
\text { Ajzen, 1991; Mathieson, 1991; Taylor \& } \\
\text { Todd, 1995a, 1995b) }\end{array}$ \\
$\begin{array}{l}\text { The senior management } \\
\text { of this business has } \\
\text { been helpful in the use } \\
\text { of the system. }\end{array}$ & $\begin{array}{l}\text { Social Factors (Thompson, Higgins, \& } \\
\text { Howell, 1991) }\end{array}$ \\
\hline SI04 & $\begin{array}{l}\text { In general, the } \\
\text { organization has } \\
\text { supported the use of } \\
\text { the system. }\end{array}$ & $\begin{array}{l}\text { Social Factors (Thompson, Higgins, \& } \\
\text { Howell, 1991) }\end{array}$ \\
\hline
\end{tabular}

Table 5.9: Social Influence: validated measurement items from UTAUT (Venkatesh, Morris, Davis, \& Davis, 2003)

For example, when discussing the perceived 'status' of system users, one user found EDRMS usage to be a strong indicator of quality of character:

I generally think that people who use [EDRMS] really well, will also be a really on-to-it type of person. Whereas, someone who is refusing to use [EDRMS] - or using it in a bit of a moronic way, or writing really moronic metadata - is going to be one of those people you are really going to struggle with. They are going to have that individualistic style. They are not going to keep a good paper trail. They are not going to be focused on customer service.

Yet on the other hand, that 'good user status' may have had personal appeal, but it may not have broader appeal in the organizational context:

In general, I don't think the organization appreciates the value of those people. 
Furthermore, the concept of critical mass or information density came out quite clearly. If very few people use the system, the benefit of using it decreases for others: this is an aspect of social influence missed by the UTAUT measurement items. In this case, the EDRMS repository becomes only one of several other potential places for storing information, and one with a higher overhead of metadata entry. Without broad usage, the system can never become a valued asset from the standpoint of the collective memory. One participant summarized the problem:

It handles everything! It's just that nobody uses it!

Additional measurement items sourced from constructs that were purported to be included in UTAUT's social influence, but not included in the final UTAUT item list, were reintroduced into the measurement item pool. These were sourced from constructs including: social factors (Thompson et al., 1991) and image (Moore \& Benbasat, 1991). These items were retested for their loading on to social influence in the context of electronic recordkeeping. The proposed set of social influence measurement items is listed in Table 5.10. 
Social Influence (SI): Measurement Items \& Source Construct

\begin{tabular}{|c|c|c|}
\hline $\begin{array}{l}\text { Item } \\
\text { Code }\end{array}$ & Proposed Measurement Item & Source \\
\hline SI01 & $\begin{array}{l}\text { People who influence my behavior think } \\
\text { that I should use the system. }\end{array}$ & UTAUT (SI) \\
\hline SI02 & $\begin{array}{l}\text { People who are important to me think } \\
\text { that I should use the system. }\end{array}$ & UTAUT (SI) \\
\hline SI03 & $\begin{array}{l}\text { The senior management of this business } \\
\text { has been helpful in the use of the } \\
\text { system. }\end{array}$ & UTAUT (SI) \\
\hline SI04 & $\begin{array}{l}\text { In general, the organization has } \\
\text { supported the use of the system. }\end{array}$ & UTAUT (SI) \\
\hline SI05 & $\begin{array}{l}\text { I use the system because of the } \\
\text { proportion of my co-workers who use } \\
\text { the system. }\end{array}$ & $\begin{array}{l}\text { Social Factors } \\
\text { (Thompson, Higgins, \& } \\
\text { Howell, 1991) }\end{array}$ \\
\hline SI06 & $\begin{array}{l}\text { People in my organization who use the } \\
\text { system have more prestige than those } \\
\text { who do not. }\end{array}$ & $\begin{array}{l}\text { Image (Moore \& } \\
\text { Benbasat, 1991) }\end{array}$ \\
\hline SI06a & $\begin{array}{l}\text { People in my organization who use the } \\
\text { system are more reliable than those who } \\
\text { do not. }\end{array}$ & $\begin{array}{l}\text { Proposed change to } \\
\text { SI06 }\end{array}$ \\
\hline SI07 & $\begin{array}{l}\text { People in my organization who use the } \\
\text { system have a high profile. }\end{array}$ & $\begin{array}{l}\text { Image (Moore \& } \\
\text { Benbasat, 1991) }\end{array}$ \\
\hline
\end{tabular}

Table 5.10: Social Influence: proposed set of Stage 1 measurement items combining those sourced from UTAUT (Venkatesh, Morris, Davis, \& Davis, 2003) with others sourced from the literature that support themes from Stage 1's thematic analysis. 


\section{Perceived Power Security}

The fourth construct - perceived power security - represents the second aspect (i.e., the internal social aspect) of the Organizational Context component of the conceptual research model.

In the literature, the perceived power security construct initially appeared to be quite important in explaining some perceived barriers to using an electronic recordkeeping system. However, among the interview participants, the importance of 'power security' in electronic recordkeeping systems did not emerge as an important consideration. Even when emphasizing the potential risks associated with transparency, judgment, and power-loss during interviews, the majority of the interview participants appeared unconvinced as to the importance of perceived power security in explaining system use. For example, one participant explained:

I guess there is always that risk, but as long as I am happy with the standard that I produce, then I don't think that it's an issue for me. Everyone will interpret things as they interpret them, but as long as I am personally happy with my performance, then I'm comfortable with that. I understand, however, that some would fret terribly about it, and they would find it terribly, terribly difficult, and they would choose to avoid using the system.

As the above illustrates, many people see system use as just part of the job, with many seeing it instead as a beneficial source of evidence that would increase their sense of security:

If you want job security in terms of decisions that are made, you will make sure that you have a record, even if you have your own little personal hard copy sitting near you.

However, power and its use did come out as potentially important at some levels in the organization, particularly as applied to the perceived recordkeeping motivations thought to be the case at senior management levels:

Yes, I think they don't want to go on the record. Knowledge is power, and if you're not around the table, they don't want you to know what they're up to. They are probably scared of making records that people could go back and look at. 
The emergent themes from the interview coding are shown in Table 5.11. Note that where the most referenced code - Fear of Judgement - ranked quite highly, many of those comments were in response to strongly probing questions and reflected a lack of personal concern and fear. What did come out was that perceived power security was not considered a direct problem or threat among the 12 interview participants. However, the majority agreed that they could understand a level of concern in others.

\begin{tabular}{lcc} 
Interview Codes: Perceived Power Security & Sources & References \\
\hline Fear of judgement & 12 & 39 \\
Reputational insurance & 11 & 19 \\
Knowledge is power & 2 & 4 \\
\hline TOTAL & 12 & 62
\end{tabular}

Table 5.11: Perceived Power Security: frequency of NVivo node-coding by number of interview participants (sources) and total number of reference quotations (references).

The perceived power security measurement items used by Ong et al. (2005) are reproduced in Table 5.12 as they were applied to the intention to use a knowledge management system.

Perceived power security (PPS): Items and Source Construct

\begin{tabular}{|l|l|l|}
\hline $\begin{array}{l}\text { Item } \\
\text { Code }\end{array}$ & Measurement Item & $\begin{array}{l}\text { Source } \\
\text { Construct }\end{array}$ \\
\hline PPS01 & $\begin{array}{l}\text { I don't worry that the KMS system will } \\
\text { affect my influence in the work environment. }\end{array}$ & $\begin{array}{l}\text { Ong, Lai, Wang, } \\
\text { \& Wang, 2005) }\end{array}$ \\
\hline PPS02 & $\begin{array}{l}\text { I don't worry that the KMS will affect my } \\
\text { power in the work environment. }\end{array}$ & $\begin{array}{l}\text { (Ong, Lai, Wang, } \\
\text { \& Wang, 2005) }\end{array}$ \\
\hline
\end{tabular}

Table 5.12: Perceived Power Security: validated measurement items from the literature (Ong, Lai, Wang, \& Wang, 2005).

Given that the original measurement items were targeted at a knowledge management systems (KMS) - a class of system that focuses on capturing employees' knowledge, as opposed to capturing their documents and records the measurement items were adjusted to reflect the context of an electronic 
recordkeeping system. The resulting set of adjusted perceived power security measurement items is shown in Table 5.13.

Perceived power security (PPS): Items and Source Construct

\begin{tabular}{|l|l|l|}
\hline $\begin{array}{l}\text { Item } \\
\text { Code }\end{array}$ & Measurement Item & Source Construct \\
\hline PPS01 & $\begin{array}{l}\text { I don't worry that the electronic record- } \\
\text { keeping system will affect my influence in } \\
\text { the work environment. }\end{array}$ & $\begin{array}{l}\text { Adapted from Ong, } \\
\text { Lai, Wang, and Wang } \\
(2005)\end{array}$ \\
\hline PPS02 & $\begin{array}{l}\text { I don't worry that the electronic record- } \\
\text { keeping system will affect my power in } \\
\text { the work environment }\end{array}$ & $\begin{array}{l}\text { Adapted from Ong, } \\
\text { Lai, Wang, and Wang } \\
(2005)\end{array}$ \\
\hline
\end{tabular}

Table 5.13: Perceived Power Security: proposed set of Stage 1 measurement items consisting of items adjusted from the literature.

\section{Perceived Value of Records (PVR)}

The perceived value of records is the final independent variable construct in the conceptual research model, and it represents the Knowledge Interpretation component. Perceived value of records is also a new construct being further developed for this research. Lewis et al. (2005, p. 391) recommend that construct formation includes three initial pieces of information: the premise, a conceptual definition, and a list of construct dimensions. Accordingly, the perceived value of records was initially defined ${ }^{4}$ as "a user's belief that knowledge artifacts (e.g., written documents, letters, emails, etc.) have value beyond the current application and are worthy of storing for the future" (Lewellen, 2013; Lewellen, Hooper, \& Oliver, 2013).

The second objective of the interview stage focused on capturing the conceptual dimensions of the perceived value of records. The codes/themes that emerged from the interview transcripts, along with the number of sources and the total references, are presented in Table 5.14.

The results of the perceived value of records thematic analysis were then considered through the lens of the Records Continuum Model (Figure 2.3, p. 24), particularly the continuum of evidentiality. With this in mind, the emergent interview themes were able to be grouped into three main dimensions:

\footnotetext{
${ }^{4}$ For the final refined definition of Perceived Value of Records, see Section 8.2.3, p. 201.
} 


\begin{tabular}{lcc} 
Interview Codes: Perceived Value of Records & Sources & References \\
\hline Need for 'organizational memory' & 12 & 47 \\
Ownership of accountability & 12 & 34 \\
Achieved recordkeeping maturity (information culture) & 11 & 55 \\
Evidence of personal accomplishment & 9 & 24 \\
Legislative compliance & 9 & 22 \\
Necessity for effective business process & 9 & 19 \\
Evidence of use of public funds & 9 & 15 \\
Capture of societal history & 7 & 15 \\
Collaboration between teams & 7 & 14 \\
Protection against staff turnover & 7 & 10 \\
\hline TOTAL & 12 & 255
\end{tabular}

Table 5.14: Perceived Value of Records: frequency of NVivo node-coding by number of interview participants (sources) and total number of reference quotations (references).

ownership of accountability, need or value of 'organizational memory', and need for reliable evidence. These are discussed in more detail in below:

Ownership of accountability The first dimension of the perceived value of records considered was the role of records to trace accountability and the importance of employee ownership - the responsibility - to create those records. The recordkeeping standard clearly states:

It is particularly important that the policy obliges all employees to create and maintain records that meet the legal, regulatory, fiscal, operational, and archival/historical needs of the organization (ISO 15489.1, 2002, p. 1).

With that in mind, the ability of employees to understand why accountability is important, and to personally own that need for accountability, has emerged from the interviews as one of the major components that is likely to impact the perceived value of records. Many of the interview participants correctly identified the need for maintaining records, particularly for legislative compliance reasons or as evidence for the proper use of public funds. One interview participant summed it up aptly by recognizing the value of recordkeeping. 
As part of an open and transparent society, where the citizens deserve the right to know what the government is doing.... Records assume an importance for that reason; that if need be, citizens can trace back why decisions were made.

That said, it came through very clearly that many employees do not yet own the need to participate in creating that 'transparent society' through good personal recordkeeping. Many comments reflected this interpretation, including:

I just think that the organization has bigger fish to fry.

There is no management champion of it [EDRMS] by any means.

The last thing he wants to know about is actually filing anything.

This apparent lack of ownership at a personal level of the need to maintain organizational accountability is expected to impact the perceived value of records and ultimately the intention to use an electronic recordkeeping system.

Value of organizational memory "An organization's ability to collect, store and use knowledge it has generated through experience can have important consequences for its performance" (Olivera, 2000, p. 811). This statement, if true, simultaneously provides a mechanism and outcome, as well as a basic business driver for creating, maintaining, and accessing a form of organizational memory.

Stein (1995) defined Organizational Memory as "the means by which knowledge from the past is brought to bear on present activities, thus resulting in higher or lower levels of organizational effectiveness." Walsh and Ungson (1991) also emphasized that the repositories of that organizational memory need not be limited to its records, and that organizational memory exists in 'bins' including individuals, culture, transformations, structures, and ecology. As discussed, organizational memory can exist in many guises, with records being one of the most visible, obvious, and tangible forms.

Organizational Memory is specifically mentioned in the Records Continuum Model as being part of the continuum of evidentiality. As such, the second dimension of perceived value of records can be attributed to the role that records have to play in the maintenance and transfer of information through time. 
Interview participants identified a number of different non-accountability roles that records play in the organization, including continuity - the helpful role of records during periods of high staff turnover; reuse of information - the ability to reuse information and thus provide efficiency gains to organizational processes; collaboration - between individuals, teams, and business units through a shared and trusted repository; business process - the role of records in the operation of business processes over time; and even societal history the role of records in clarifying the changes in society over time.

Interview participants had a range of views on the value of organizational memory. Comments were sometimes quite extreme:

I think part of it is the valuable resource. I dip into the records to find: 'What did we say last time? What kind of proforma approach to attacking an issue?' - and that's really useful!

In contrast, another participated commented:

I'm not so sure [that records are valuable] - probably not so much. In the area of IT, things are moving so fast that sometimes a decision that's 18-months old is almost irrelevant.

Thus, a user's individual contextual need to access the organizational memory - their need for a trustworthy repository and the value to them of what it contains - is also expected to impact their perceived value of records.

Need for trustworthy evidence The last dimension that emerged from interview participants concerning the perceived value of records is a more personal requirement for trustworthy evidence. This is another use of records (and more specifically, recordkeeping metadata), and could be considered separate from the broader accountability requirement or the need for the information contained in records in terms of 'organizational memory'. This is the ability to retain trustworthy records that are important primarily to the individual. Nine of the interview participants made at least 24 mentions of the importance of maintaining evidence of their personal accomplishments, with the primary motivation being the use of that evidence as leverage for promotion or for the demonstration of the ongoing delivery of value to the organization.

The need for trustworthy evidence of employees' accomplishments is highlighted in a recent case where fewer employees were going to be retained after an organizational restructuring: 
I think, possibly, the issue coming to a head now, for them, is: they don't have a record of their work very easily. And they are realizing that that could be a real problem coming up. So, I think that the value of records could hit home for a few people real soon. So, while I haven't got everything filed in the system, the key points are in there. So I'm reasonably comfortable, whereas most other people [...] are [saying], 'What are we going to rely on in terms of a record of our work?'

Another interview participant cheerfully concluded:

You know, I can sum it all up. It's all ass-covering. And asscovering is a good thing!

The primary themes of perceived value of records that emerged from the interviews can thus be linked back to the information that is required at various points time as illustrated in the Records Continuum Model (Figure 2.3, p. 24). The Records Continuum includes the use of a record as evidence, as corporate/individual memory, and finally as a collective memory for the purposes of accountability. However, this requires ownership of that collective responsibility by individuals. With that in mind, the proposed PVR measurement items, representing the construct dimensions or 'facets' of the construct (Bollen \& Lennox, 1991), have been brought together in Table 5.15. 
Perceived Value of Records (PVR): Proposed Measurement Items

\begin{tabular}{|c|c|c|}
\hline Code & Proposed Measurement Item & Dimension \\
\hline PVR01 & $\begin{array}{l}\text { Records management is everyone's } \\
\text { responsibility. }\end{array}$ & Accountability \\
\hline PVR02 & $\begin{array}{l}\text { Records management requirements } \\
\text { are a barrier to working efficiently. }\end{array}$ & Accountability \\
\hline PVR03 & $\begin{array}{l}\text { Records management is an essential } \\
\text { part of my work. }\end{array}$ & Accountability \\
\hline PVR04 & $\begin{array}{l}\text { I make sure all my work-related } \\
\text { emails are filed appropriately. }\end{array}$ & Accountability \\
\hline PVR05 & $\begin{array}{l}\text { The records repository is the } \\
\text { preferred way to share documents } \\
\text { with other teams or business units in } \\
\text { my organization. }\end{array}$ & Organizational Memory \\
\hline PVR06 & $\begin{array}{l}\text { Records are an important source of } \\
\text { information that I use regularly in my } \\
\text { job. }\end{array}$ & Organizational Memory \\
\hline PVR07 & $\begin{array}{l}\text { Particularly during times of high staff } \\
\text { turnover, records are necessary to } \\
\text { provide continuity to business } \\
\text { processes. }\end{array}$ & Organizational Memory \\
\hline PVR08 & $\begin{array}{l}\text { I save my records into the system } \\
\text { with the thought that someone in the } \\
\text { future will read them. }\end{array}$ & Organizational Memory \\
\hline PVR09 & $\begin{array}{l}\text { I rely on the recordkeeping system to } \\
\text { provide reliable evidence of my } \\
\text { personal accomplishments. }\end{array}$ & Evidence \\
\hline
\end{tabular}

Table 5.15: Perceived Value of Records: proposed set of Stage 1 measurement items as generated from the emergent interview themes and organized according to their construct dimension. 


\section{Stage 1 Summary and Conclusion}

The interview stage used thematic analysis to create a set of emergent themes associated with each of the constructs of interest.

The resulting themes were then considered in light of the measurement items published in the literature. Where an existing measurement item reflected the essence of the emergent theme, it was added to the pool of measurement items. Where the emergent themes uncovered additional dimensions of the constructs, they were transformed into new measurement items. In this way, the measurement items from the literature were validated for use in the context of electronic recordkeeping systems, while the interviews provided additional measurement items to increase the depth and breadth of measurement in the context of electronic recordkeeping.

Next, the new construct - perceived value of records - was explored using three construct dimensions borrowed from the Records Continuum Model. The emergent themes - having been found to align nicely with the proposed construct dimensions - were also converted into measurement items and added to the pool.

Finally, the process of conducting the thematic analysis independently of a predetermined structure supported the search for any additional items or factors. These additional factors, if detected, could potentially impact a user's intention to file documents in an electronic recordkeeping system in a way that is not specifically covered by the constructs of the conceptual research model. None was found; however, the convergent and discriminant validity of the final measurement items would be further tested in Stage 3.

The next stage of the research used new participants to assist in proofreading the pool of measurement items and helped to improve their wording, clarity, and quality. 


\subsection{Stage 2: Proofreading}

Stage 2 of the qualitative research phase focused on improving and aligning the measurement items and beginning the process of construct validation (Peter, 1981). Construct or content validity is defined as "the degree to which the score or scale being used [e.g., measurement items] represents the concept about which generalizations are to be made" (Bohrnstedt, 1970, p. 91, as referenced in Davis, 1989, p. 323). This stage focused on each individual measurement item in order to determine if the statement itself was both clear and easy to understand and therefore was able to measure the concept it attempted to represent.

The objectives of this stage were to:

1. identify measurement items from Stage 1 that were vague, unclear, or difficult to understand, and address those issues;

2. align all of the measurement item statements in terms of tense, grammar, and style; and

3. combine the required changes into an improved Stage 2 measurement item pool for use in later research stages.

The data collection, analysis methods, and results of Stage 2 are described in more detail below.

\subsubsection{Proofreading Methods}

Prior to developing the survey instrument (in Phase II), the measurement items must first go through an iterative refining process (Lewis et al., 2005; Moore \& Benbasat, 1991). Stage 1 in the process collected all of the measurement items from the literature along with those proposed by the researcher in order to create an initial pool of measurement items. See Section 5.2.4.

The next stage in this process (Stage 2) elicited the input from ten proofreading participants. These volunteers were given limited background concerning the research project and simply were told that the 'system' referred to was an electronic recordkeeping system. They were then asked to review 43 measurement items (presented on paper) from the Stage 1 measurement item pool. If the item was clear and understandable, and therefore required no change, the participants were asked to place a tick in front (to indicate 
active approval). If there were issues, they were invited to indicate comments, suggestions, or changes as appropriate.

The ten proofreading participants were selected from two New Zealand public sector organizations and represented three different business units. Two participants represented a team that is responsible for the development of internal organizational research projects and surveys. They provided particularly detailed feedback on phrasing, identifying (from the vantage point of their experience in organizational research) potential 'double-barreled' questions and problematic word-choices that could be confusing to potential survey participants.

In total, 90 issues were raised that affected 32 measurement items, with many of those issues reiterating the same or similar problems. See Table 5.16 for a breakdown of the proofreading feedback.

\begin{tabular}{lcc} 
Measurement Item Feedback & Issues & Questions \\
\hline No change required & 0 & 12 \\
Minor change (e.g., tense, word alignment) & 34 & 19 \\
Major change (e.g., rewording, clarification, phrasing) & 56 & 26 \\
Additional measurement item suggested & 0 & 2 \\
\hline TOTAL & 90 & 45
\end{tabular}

Table 5.16: Feedback on Measurement Items: the frequency of issues/ suggestions raised (Issues) and the number of affected measurement items (Questions) raised during the Stage 2 proofreading activity.

\subsubsection{Proofreading Analysis and Results}

The proofreading feedback, consisting of both issues and suggestions, was then transcribed onto a single master copy. See Appendix B on p. 235. By creating a single master document, all of the issues and suggestions could be viewed simultaneously and considered in context with other feedback. This was an important step in identifying a shared view and common understanding of the items, including how best to represent their meaning.

A number of suggestions dealt with the need to standardize the word system. Sometimes it was referred to as 'the system', or the 'electronic recordkeeping system,' or the 'records repository,' etc. It was suggested that statement should either refer to the organization's specific system name, or 
else it be set up as a variable name - e.g., the system - where the variable is defined earlier for clarity. There were 19 instances where participants highlighted this specific issue, and it was agreed that the EDRMS would be referred to as 'the system' where required.

Other issues raised (by frequency) included suggestions for specific wordchoice (17), alignment of the tense for all measurement items (13), phrasing suggestions (12), and the need for additional clarification (9).

The two measurement items associated with perceived power security were found to be the most confusing, with six participants taking issue with one or more parts of each measurement item statement. The result was that the existing items were rewritten for clarity. In addition, two new measurement items that reflect both the authoritative and allocative aspects of power were introduced to the pool. These two aspects of power were discussed in the literature review and provide additional dimensions to this construct.

Problematic measurement items were then reworked to incorporate the best of the feedback. The final wording was then checked for tense and clarity. The resulting pool of measurement items containing the updated wording is provided in Table 5.17 .

Table 5.17 - Stage 2 Measurement Item Wording

\begin{tabular}{|c|c|}
\hline Item Code & Measurement Item \\
\hline PE01* & I find the system useful in my job. \\
\hline PE02 & $\begin{array}{l}\text { Using the system enables me to accomplish tasks more } \\
\text { quickly. }\end{array}$ \\
\hline PE03 & Using the system increases my productivity. \\
\hline PE04* & $\begin{array}{l}\text { Using the system increases my chances of getting recognition } \\
\text { in the workplace - e.g., contributes to promotion chances. }\end{array}$ \\
\hline PE05* & $\begin{array}{l}\text { Using the system's Search Function allows me to locate } \\
\text { records effectively. }\end{array}$ \\
\hline PE06* & $\begin{array}{l}\text { When I store a record in the system, I am confident that I } \\
\text { will be able to retrieve it in the future. }\end{array}$ \\
\hline PE07* & $\begin{array}{l}\text { The system effectively manages security to the level required } \\
\text { for my sensitive records. }\end{array}$ \\
\hline PE08* & I find that the records are effectively organized in the system. \\
\hline PE09* & I have found the system to be reliable. \\
\hline PE10* & I know when I am supposed to use the system in my job. \\
\hline & ... continued on next page \\
\hline
\end{tabular}


Table 5.17 - continued from previous page

\begin{tabular}{|c|c|}
\hline Item Code & Measurement Item \\
\hline PE11* & $\begin{array}{l}\text { My organization uses the system as a way to achieve one or } \\
\text { more of its goals. }\end{array}$ \\
\hline PE12* & $\begin{array}{l}\text { The system's ability to integrate with my other computer } \\
\text { applications ultimately saves me time in my job. }\end{array}$ \\
\hline PE13 & I have noticed that the system is often slow in its operation. \\
\hline PE14* & I use the system to report on the status of various records. \\
\hline EE01* & $\begin{array}{l}\text { I find the system's interface (e.g., its menus and layout) to } \\
\text { be clear and understandable. }\end{array}$ \\
\hline EE02* & I find it easy to become skillful at using the system. \\
\hline EE03* & I find the system easy to use in my job. \\
\hline EE04* & Learning to operate the system was easy for me. \\
\hline EE05* & I use the system often enough to maintain my skill in its use. \\
\hline EE06* & $\begin{array}{l}\text { The process of contributing a record to the system (i.e., } \\
\text { electronic filing) is easy and straightforward. }\end{array}$ \\
\hline EE07 & I have no trouble searching for records in the system. \\
\hline EE08* & $\begin{array}{l}\text { I can learn how to do administrative tasks in the system } \\
\text { without difficulty. }\end{array}$ \\
\hline EE09* & $\begin{array}{l}\text { I can access the system directly from other computer appli- } \\
\text { cations - e.g., directly from Microsoft Office. }\end{array}$ \\
\hline EE10* & $\begin{array}{l}\text { The system is flexible enough to support both my needs as } \\
\text { well as the organization's requirements. }\end{array}$ \\
\hline SI01 & $\begin{array}{l}\text { People who influence my behavior think that I should use } \\
\text { the system. }\end{array}$ \\
\hline SI02 & $\begin{array}{l}\text { People who are important to me think that I should use the } \\
\text { system. }\end{array}$ \\
\hline SI03* & $\begin{array}{l}\text { The senior management of this organization support the use } \\
\text { of the system. }\end{array}$ \\
\hline SI04* & $\begin{array}{l}\text { In general, the organization has supported the use of the } \\
\text { system. }\end{array}$ \\
\hline SI05* & $\begin{array}{l}\text { I use the system because many of my co-workers also use the } \\
\text { system. }\end{array}$ \\
\hline SI06* & $\begin{array}{l}\text { People in my organization who use the system are more highly } \\
\text { regarded than those who do not. }\end{array}$ \\
\hline $\mathrm{SIO}^{*}$ & $\begin{array}{l}\text { People in my organization who use the system are more } \\
\text { reliable than those who do not. }\end{array}$ \\
\hline
\end{tabular}


Table 5.17 - continued from previous page

\begin{tabular}{|c|c|}
\hline Item Code & Measurement Item \\
\hline SI08* & $\begin{array}{l}\text { People in my organization who regularly use the system } \\
\text { acquire a higher profile. }\end{array}$ \\
\hline $\mathrm{PPS} 1^{*}$ & $\begin{array}{l}\text { Placing my documents in the system - where other people } \\
\text { may view them - may positively affect my reputation. }\end{array}$ \\
\hline PPS02* & $\begin{array}{l}\text { I worry that by recording my knowledge in the system, I will } \\
\text { be less valuable to the organization as a source of knowledge. }\end{array}$ \\
\hline PPS03* & $\begin{array}{l}\text { By placing my records in the system, I feel that I have more } \\
\text { control over them. }\end{array}$ \\
\hline PPS04* & $\begin{array}{l}\text { I am concerned that by putting my documents into the system, } \\
\text { there is a potential for people to judge my work. }\end{array}$ \\
\hline PVR01 & Records management is everyone's responsibility. \\
\hline PVR02 & $\begin{array}{l}\text { Records management requirements are a barrier to working } \\
\text { efficiently. }\end{array}$ \\
\hline PVR03 & Records management is an essential part of my work. \\
\hline PVR04 & I make sure all my work-related emails are filed appropriately. \\
\hline PVR05* & $\begin{array}{l}\text { The system is the preferred way to share documents with } \\
\text { other teams or business units in my organization. }\end{array}$ \\
\hline PVR06* & $\begin{array}{l}\text { I regularly refer to records to obtain information needed in } \\
\text { my job. }\end{array}$ \\
\hline PVR07* & $\begin{array}{l}\text { Particularly during times of high staff-turnover, records are } \\
\text { necessary to provide continuity to my organization's business } \\
\text { processes. }\end{array}$ \\
\hline PVR08 & $\begin{array}{l}\text { I save my records into the system with the thought that } \\
\text { someone in the future will read them. }\end{array}$ \\
\hline PVR09 & $\begin{array}{l}\text { I rely on the system to provide reliable evidence of my personal } \\
\text { accomplishments. }\end{array}$ \\
\hline
\end{tabular}

Table 5.17: Measurement Items after Stage 2 proofreading. Those marked with an asterisk $\left(^{*}\right)$ were improved as a result of the Stage 2 proofreading feedback. 


\subsection{Stage 3: Card Sorting}

The final stage of the qualitative phase (Stage 3) explored the measurementitem-to-construct relationship and used some quantitative techniques to verify that this relationship contained a shared interpretation, understanding, and meaning amongst a sample of participants. To this point, the relationship between a measurement item and its parent construct had merely been proposed by the researcher. This stage sought to lend reliability and validity to that assigned relationship by incorporating the views of others.

The objectives of this stage were to:

1. test the convergent validity of the measurement-item-to-construct relationship and thereby verify that the proposed relationship is similarly interpreted and shared by other participants;

2. test the discriminant validity of the measurement-item-to-construct relationship and thereby verify that the provided number of constructs are both sufficient and necessary to explain the number of measurement items that emerged from Stages 1 and 2; and

3. recategorize and adjust those measurement items and/or constructs as required to maximize the convergent and discriminant validity measures, should any of the proposed measurement item relationships change as a result of the analysis.

The technique selected to explore the relationship between the construct and its measurement items was card sorting (Moore \& Benbasat, 1991; Hinkle, 2008). The goal of this stage was to verify that the pool of measurement items loaded appropriately onto one (and only one) of the constructs in the research model. Two methods of card sorting - closed card sorting and open card sorting - were employed to measure these two different aspects of validity (Boudreau et al., 2001; Rugg \& McGeorge, 2005). Items demonstrating lower reliability were candidates for elimination, as these items would likely have low explanatory power in the final research model (Hinkin, 1998; Moore \& Benbasat, 1991).

A sample of 24 participants (12 for each variation) were recruited to participate in card sorting in order to obtain a recommended sample size to measure validity (J. R. Wood \& L. E. Wood, 2008). The participants were drawn from a single New Zealand public sector organization, but represent a number of different levels within the organizational hierarchy, including a 
director, several functional managers, and a range of team leaders and workers from various functional areas who were attending an out-of-town conference together.

\title{
5.4.1 Card Sorting Introduction Procedure
}

Participants were all provided with a similar introduction to the stated goals of the research project and the purpose of the card sorting stage.

\section{Example Card Sort}

After the introduction, a simple card sort example was completed by the researcher in front of the participant to demonstrate the purpose of the card sort validation. The following food items were placed on cards and described as follows:

Let's take a look at an example of how people may sort a group of items. Consider these common foods.

\author{
Spinach \\ Beef \\ Peanut butter \\ Pork \\ Lettuce \\ Silverbeet \\ Beans and lentils
}

Generally, people will sort these into three groups.

\begin{tabular}{ccc} 
Group 1 & Group 2 & Group 3 \\
\hline Spinach & Peanut butter & Beef \\
Lettuce & Beans and lentils & Pork \\
Silverbeet & &
\end{tabular}


Now, if I were to tell you the categories I was studying, it may influence how you view these groups. For example, if I were studying vegetarian and non-vegetarian foods, most people would sort them like this:

\begin{tabular}{cc} 
Vegetarian & Non-Vegetarian \\
\hline Spinach & Beef \\
Lettuce & Pork \\
Silverbeet & \\
Peanut butter & \\
Beans and lentils &
\end{tabular}

But if I were studying high-protein and low-protein foods, most people would sort them like this:

\begin{tabular}{cc} 
Low-Protein & High-Protein \\
\hline Spinach & Beef \\
Lettuce & Pork \\
Silverbeet & Peanut butter \\
& Beans and lentils
\end{tabular}

Now, if I were to give you no categories at all, and leave it to you to sort, I might see 2 or 3 categories that may contain different items depending on how people interpret the categories. But in all cases for the above example, the meats and leafy greens are always clustered, while the Peanut butter and Beans and lentils migrate around. If I drop the Peanut butter and Beans and lentils from my list of measurement items, then I am left with two unambiguous groupings that appear to work in all situations:

\begin{tabular}{cc} 
Group A & Group B \\
\hline Spinach & Beef \\
Lettuce & Pork \\
Silverbeet &
\end{tabular}


You may notice that some measurement items in my project are easy to categorize, and some are more difficult. That's to be expected, and those may be candidates for dropping from the list. Do your best.

At this point, the participant would either be asked to sort the 45 measurement item statements (cards) against the research model constructs (i.e., the closed card sort) or else they were asked to make their own categories and category titles (the open card sort).

\subsubsection{Closed Card Sorting Methods}

The first variation was closed card sorting (Spencer \& Warfel, 2004). First, the participants were provided with the research model's construct categories written on cards laid across the top of a table:

$$
\begin{gathered}
\text { Performance Expectancy (Usefulness) } \\
\text { Effort Expectancy (Ease of use) } \\
\text { Social Influence } \\
\text { Perceived Power Security } \\
\text { Perceived Value of Records }
\end{gathered}
$$

The construct categories were briefly described. It was pointed out that software generally needs to be both useful and easy to use for someone to be willing to use it. Does the technology do what is expected and in a way that makes sense? If so, then the software itself is designed well.

EDRMS are also used in the context of an organization, so social influences may be important. Managers and peers may/may not encourage and support its use, and these external social pressures may impact a user's willingness to use it. Furthermore, users may have internal/personal concerns, either over control of the files themselves or over knowledge-is-power aspects of information being shared in a central repository. These are captured under perceived power security.

Finally, perceived value of records was introduced, as even if the software is well designed and people support its use, if the user doesn't value records, they still may not participate.

At that point, participants were provided with the 45 measurement items printed on cards. These cards were shuffled; however, the first 2-3 cards had been chosen to be unambiguous - e.g., EE03: I find the system easy to use in my job. The participant was then left to do their sort (about 15 minutes). 
When the sort was completed, the participant was asked to recheck their sorting. They were also asked if any cards were particularly difficult to place. The item codes - e.g., PE04, PVR07, etc. - were noted on the reverse side of the cards to facilitate data capture, and the resulting data were recorded in a notebook.

\subsubsection{Open Card Sorting Methods}

The second variation was open card sorting (Faiks \& Hyland, 2000). In this variation, participants were asked to sort and develop their own categories from the available item pool. This was a test of discriminant validity (measuring that a factor unambiguously loads onto a single construct). ${ }^{5}$

The open card sort participants were shown the same introduction example sort and were similarly introduced to the goals of the research project; however, the research model (and construct categories) were deliberately withheld.

The open card sort was much more challenging for participants, who generally had to approach the sorting iteratively, and the time-to-sort was approximately double that of the closed card sort. As such, the participants selected for the open card sort were generally individuals who were known to have a longer attention span and a greater willingness to participate.

The participants were told that between 5-7 categories were expected, and that the measurement items would not necessarily be evenly distributed between the categories. The range of 5-7 categories was specified to provide leeway for participants to use their own logical grouping, while limiting the number of categories to a reasonable number. Even so, one participant apologetically created 8 categories. The participants were also warned that positive and negative statements could be in the same category - e.g., 'negative statements' was not an expected category. They were then provided with a stack of empty cards and a pencil, so that they could create working titles for their categories as they sorted.

When the sort was completed, the participants were asked to recheck their work. They were also asked if any cards were particularly difficult to place, and then the resulting data were recorded in the research notebook. All participants were thanked for their time and participation.

\footnotetext{
${ }^{5}$ Some of the established and validated construct measurement items from the literature had been altered or added to, taking into account the new situation-specific information from the interview stage. This additional validation provided an opportunity to check the nomological validity for some of the more established constructs and their associated measurement instruments (Boudreau et al., 2001, p. 12).
} 


\subsubsection{Card Sorting Analysis and Results}

The card sorting data were entered into a LibreOffice 4 Calc spreadsheet with two worksheet tabs, one to capture the open card sort data and the other to capture the closed card sort data. The data were entered by participant and by group, using " 1 " to denote a positive sort in that group, and "0" to represent that item's absence. See Figure 5.1 below.

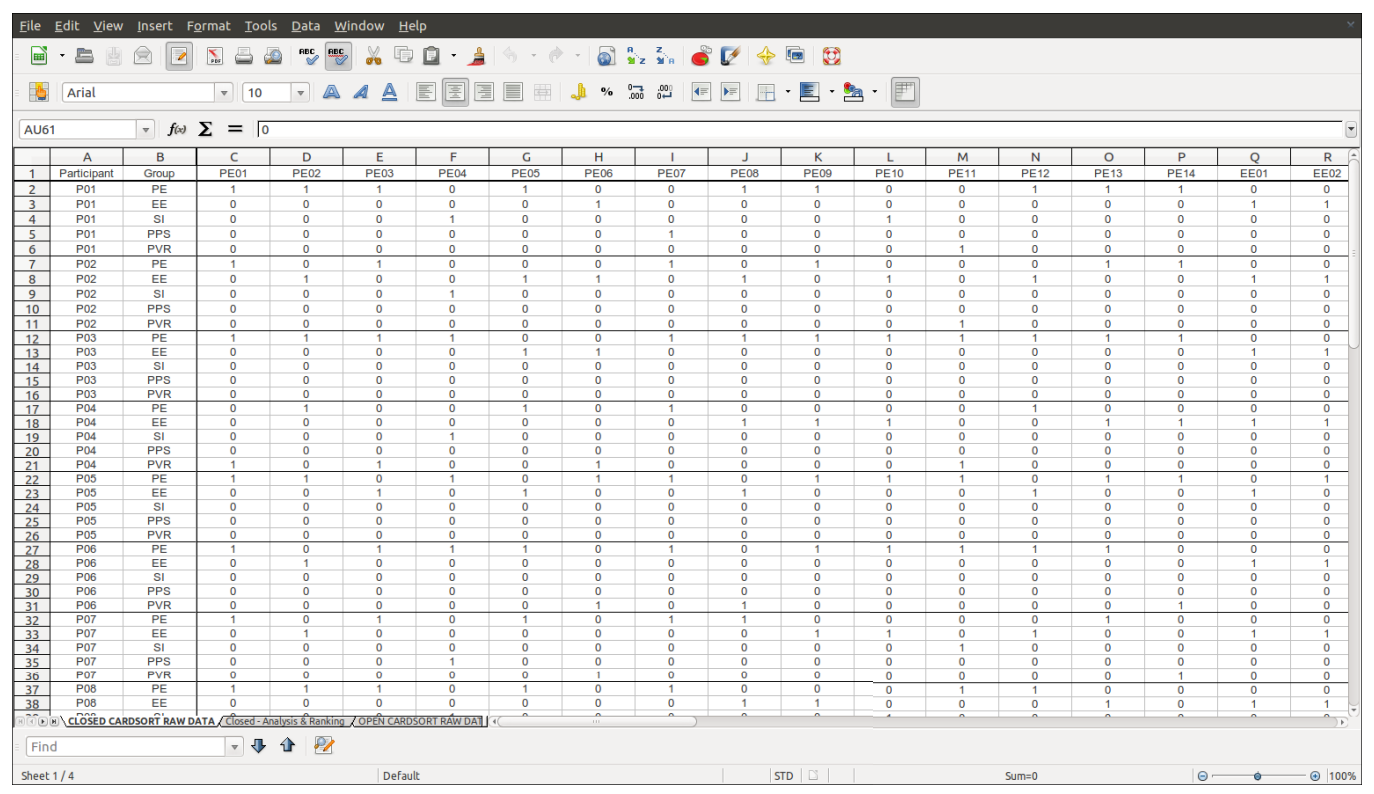

Figure 5.1: Card sorting data were captured as 1 or 0 to facilitate analysis

Once the data were entered (and checked and double-checked), the columns recording the Participant and Group columns were deleted and the data were saved as a simple text file (e.g., open.txt) so as to retain their grouping structure without any links to participant or construct. The data were then analyzed using a cluster analysis matrix that featured the use of a Jaccard coefficient analysis and an automated graphical visualization of the output in the form of a dendrogram or 'tree diagram' (Sanchez, 2012a; Salmoni, 2012; Hinkle, 2008; Faiks \& Hyland, 2000). The analysis software used was $R$ Statistics (R Core Team, 2013) running on an Ubuntu 12.04 LTS Linux platform. 


\section{Closed Card Sort Analysis and Results}

The closed card sort data were analyzed in two ways: the creation of the dendrogram as an output of the statistical cluster analysis, and the creation of a matrix describing the agreement frequency or likelihood that a measurement item was grouped in the expected construct category (stated as a percentage).

The initial dendrogram of the closed card sort was configured to create 5 clusters (indicated with boxes) in anticipation of alignment with the five constructs under investigation. See Figure 5.2. It was hoped that the 5 clusters would align well with the research model's construct categories, which would indicate acceptable discriminant validity. The resulting dendrogram revealed that the first four clusters of measurement items were solidly clustering as anticipated - i.e., SI, EE, PVR, and PPS. ${ }^{6}$ However, Figure 5.2 also revealed a cluster of weaker measurement items with statistically ambiguous clustering in the right-hand cluster.

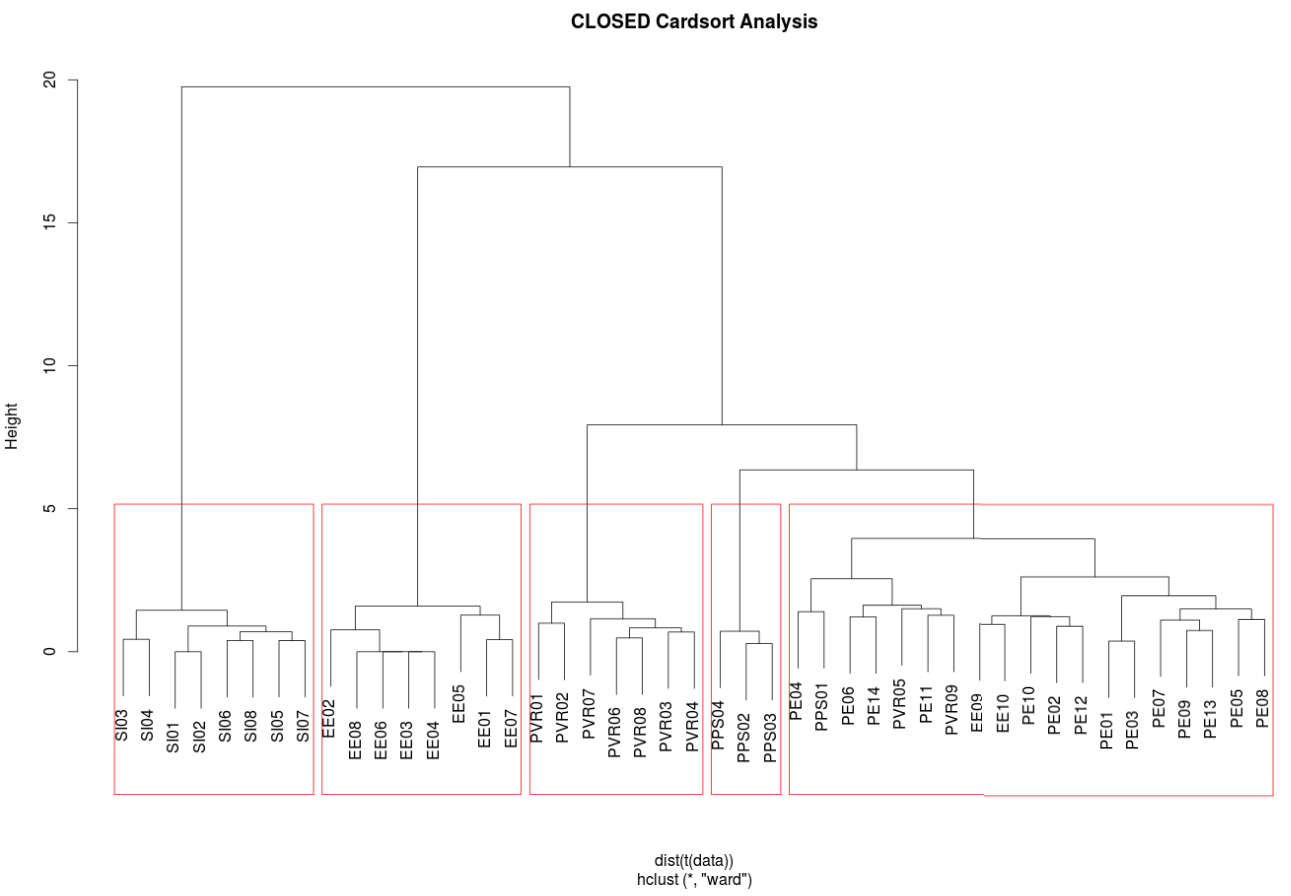

Figure 5.2: Dendrogram of the unadjusted (raw) closed card sort data

\footnotetext{
${ }^{6}$ Note that the items were coded using the initials of the construct names, where: SI $=$ Social Influence; EE = Effort Expectancy, PVR = Perceived Value of Records, PPS = Perceived Power Security, and PE = Performance Expectancy.
} 
To assess the relationship strength of the measurement items, a simple matrix was created to describe the likelihood that a measurement item was sorted into the expected construct category. So, if an item were sorted in the expected category 9 times out of 12 sorts, it would be awarded an agreement frequency of 0.75 . See Table 5.18 for the ranked agreement frequency of the raw closed card sort data.

Measurement Items \& Agreement Frequency with Construct Categories

\begin{tabular}{|ll|ll|ll|ll|ll|}
\hline PE01 & 0.83 & EE03 & 1.00 & SI01 & 1.00 & PPS02 & 1.00 & PVR03 & 0.92 \\
PE03 & 0.75 & EE04 & 1.00 & SI02 & 1.00 & PPS03 & 0.92 & PVR06 & 0.83 \\
PE07 & 0.75 & EE06 & 1.00 & SI05 & 0.92 & PPS04 & 0.83 & PVR08 & 0.83 \\
PE09 & 0.75 & EE08 & 1.00 & SI06 & 0.92 & PPS01 & 0.42 & PVR01 & 0.67 \\
PE05 & 0.67 & EE01 & 0.92 & SI03 & 0.83 & & & PVR04 & 0.67 \\
PE13 & 0.67 & EE02 & 0.92 & SI04 & 0.83 & & & PVR07 & 0.67 \\
PE02 & 0.58 & EE07 & 0.83 & SI07 & 0.83 & & & PVR02 & 0.58 \\
PE12 & 0.58 & EE05 & 0.75 & SI08 & 0.83 & & & PVR09 & 0.33 \\
PE14 & 0.58 & EE09 & 0.67 & & & & & PVR05 & 0.17 \\
PE11 & 0.42 & EE10 & 0.42 & & & & & & \\
PE08 & 0.33 & & & & & & & & \\
PE10 & 0.33 & & & & & & & & \\
PE04 & 0.25 & & & & & & & & \\
PE06 & 0.25 & & & & & & & & \\
\hline
\end{tabular}

Table 5.18: Closed card sort: the unadjusted (raw) observed agreement frequency of measurement items against their related construct category. Those that are $<0.50$ (in italic) lack sufficient discriminant validity.

To address measurement items with apparent poor construct loading, any closed card sort measurement item receiving an agreement frequency of $<0.50$ was both qualitatively and quantitatively reviewed in an attempt to understand and address the poor loading in light of the measurement item statement and its relationship with other statements in the dendrogram or agreement frequency matrix. The 0.50 threshold was selected at this stage as a conservative cut-off to maximize the number of remaining measurement items (Straub, 1989), although some studies go as low as 0.35 (Lewis et al., 2005). Any measurement items achieving an agreement frequency $\geq 0.50$ were retained unchanged at this stage, but their suitability as measurement items would be validated again during the final survey data analysis. 
At this point, the poor performers (those achieving $<0.50$ ) were evaluated, adjusted, or dropped as follows.

PE06 (0.25) When I store a record in the system, I am confident that I will be able to retrieve it in the future.

This item loaded more highly on to EE (reflecting the confidence or 'perceived ease' of retrieval) and PVR (reflecting the need and/or value of the record in the future) at 0.33 each. The expected interpretation of PE06 was as a reflection of the stability and reliability of the software i.e., will the system work in the future? However, the aspect of interest is the importance of the preservation and retrievability of the record. As such, this item was moved to PVR and rephrased to read: I save my records into the system with the thought that someone in the future will read them.

Change: 1) changed text to: "I save my records into the system with the thought that someone in the future will read them."; and 2) changed item coding to PVR11, and removed PE06 from PE item pool.

PE04 (0.25) Using the system increases my chances of getting recognition in the workplace - e.g., contributes to promotion chances.

This item strongly loaded on to SI (0.58). The expected interpretation was on PE - a means-to-an-end; however, the effect was indirect and was at least once removed from other direct performance aspects of the system. Most participants saw this item as more about people and less about the system, and many noted it as difficult to sort.

Change: changed item coding to SIO9, and removed PE04 from PE item pool.

PE10 (0.33) I know when I am supposed to use the system in my job.

This item loaded more highly on to EE (0.42) - a reflection of the effort associated with having to figure out when to use the system. The intention was to discover the strength of the connection between using the system and one's job (PE). However, the emphasis on clarity of when the system is to be used would also logically reduce effort. This item was ambiguous.

Change: removed item PE10 from pool.

PE08 (0.33) I find that the records are effectively organized in the system. This item loaded more highly on to EE (0.58). The intention was to 
measure the effectiveness of the classification schema as an aspect of usefulness (PE); however, an effective classification schema would also make the system easier-to-use (EE). Furthermore, 8 of the original interview participants made 24 references to the challenges associated with document classification, with many expressing 'confusion'. A more logical classification system would be expected to increase the system's ease-of-use.

Change: 1) changed text to: "I find that the records are logically organized in the system"; and 2) changed item coding to EE11, and removed PE08 from PE item pool.

PE11 (0.42) My organization uses the system as a way to achieve one or more of its goals.

This item loaded more highly on to PVR (0.50). The intention was to link achievement of goals to the usefulness of the system (PE); however, the more common interpretation appeared to be that linking organizational goals to the use of the system was a reflection of the perceived value of the records (PVR). Similar to PE04, PE11 was also an indirect measure of the system performance and was therefore ambiguous. To strengthen the relevance to PVR, the statement was rephrased to read: My organization relies on records as a way to achieve one or more of its goals.

Change: 1) changed text to: "My organization relies on records as a way to achieve one or more of its goals."; and 2) changed item coding to PVR10, and removed PE11 from PE item pool.

EE10 (0.42) The system is flexible enough to support both my needs as well as the organization's requirements.

This item loaded more highly on to PE (0.50). In the interviews, many participants complained of the difficulty of working in 'someone else's' classification (EE); however, the more direct interpretation reflects on the capability of the system design $(\mathrm{PE})$.

Change: changed item coding to PE15 and removed EE10 from EE item pool.

PPS01 (0.42) Placing my documents in the system - where other people may view them - may positively affect my reputation.

This item loaded more highly on to SI (0.50). The intention was to capture the level of concern over the change-of-power associated 
with the transparency that comes with a central repository for all documents. However, more participants saw this reputation as being formed externally and attributed by others in the organization (SI).

Change: changed item coding to SI10, and removed PPS01 from PPS item pool.

PVR05 (0.17) The system is the preferred way to share documents with other teams or business units in my organization.

This item loaded more highly on to PE (0.42). The intention was to determine how well an organization safeguards its records - e.g., by sharing access in a controlled manner in the system as opposed to removing records from control and emailing them, etc. However, this item was too indirect, and more participants viewed this as a straightforward capability of the system (PE). This item was ambiguous.

Change: removed item PVR05 from pool.

PVR09 (0.33) I rely on the system to provide reliable evidence of my personal accomplishments.

This item loaded equally well on to PE (0.33). The interviews revealed the importance of records to the individual as evidence of their personal accomplishments and this item was expected to reflect PVR well. However, the phrasing of this item put more emphasis on the system in that role (PE). Rephrasing the statement to: 'I have used records to provide reliable evidence of my personal accomplishments' was intended to fix that ambiguity.

Change: 1) changed text to: "I have used records to provide reliable evidence of my personal accomplishments."

It can be seen that several of the poor-performing measurement items were found to be ambiguous across all construct categories. As such, PE06, PE10, and PVR05 were removed from the measurement item pool. However, five of the measurement items were found to have achieved $\mathrm{a} \geq 0.50$ loading on to different constructs - PE04 (SI: 0.58), PE08 (EE: 0.58), PE11 (PVR: 0.50), EE10 (PE: 0.50), and PPS01 (SI: 0.50). These were recoded to the new construct and removed from their original construct pool. In addition, some items (PVR09, EE11, and PVR10) were reworded in an effort to improve their loading on to their new construct category. ${ }^{7}$

\footnotetext{
${ }^{7}$ Note: PVR09 and PVR11 - indicated in Table 5.19 in italics - did not meet the $\geq 0.50$ threshold. However, they had their text adjusted (as had EE11 and PVR10), which was
} 
In order to measure the impact of the recoding on the measurement-itemto-construct relationship, the above changes were applied to the original closed card sort data, resulting in a new adjusted agreement frequency matrix as applied to the closed card sort data. See Table 5.19 below.

Measurement Items and Agreement Frequency with Construct Categories

\begin{tabular}{|ll|ll|ll|ll|ll|}
\hline PE01 & 0.83 & EE03 & 1.00 & SI01 & 1.00 & PPS02 & 1.00 & PVR03 & 0.92 \\
PE03 & 0.75 & EE04 & 1.00 & SI02 & 1.00 & PPS03 & 0.92 & PVR06 & 0.83 \\
PE07 & 0.75 & EE06 & 1.00 & SI05 & 0.92 & PPS04 & 0.83 & PVR08 & 0.83 \\
PE09 & 0.75 & EE08 & 1.00 & SI06 & 0.92 & & & PVR01 & 0.67 \\
PE05 & 0.67 & EE01 & 0.92 & SI03 & 0.83 & & & PVR04 & 0.67 \\
PE13 & 0.67 & EE02 & 0.92 & SI04 & 0.83 & & & PVR07 & 0.67 \\
PE02 & 0.58 & EE07 & 0.83 & SI07 & 0.83 & & & PVR02 & 0.58 \\
PE12 & 0.58 & EE05 & 0.75 & SI08 & 0.83 & & & PVR10 & 0.50 \\
PE14 & 0.58 & EE09 & 0.67 & SI09 & 0.58 & & & PVR09 & 0.33 \\
PE15 & 0.50 & EE11 & 0.58 & SI10 & 0.50 & & & PVR11 & 0.33 \\
\hline
\end{tabular}

Table 5.19: Closed card sort: the adjusted observed agreement frequency of measurement items on their related construct category.

The adjusted closed card sort data were also used to create an adjusted closed card sort cluster dendrogram. See Figure 5.3. The new analysis revealed that the number of measurement items with statistically ambiguous clustering had greatly decreased. The improvement reflects the removal of lowperforming items and the recategorizing of other items to more appropriate construct categories; however, it could not reflect how well the improved wording would affect loading without conducting additional card sorting activities. This was reviewed again during the survey data analysis stage.

Nonetheless, the closed card sort results were considered sufficient to designate a pool of measurement items suitable for use in the quantitative survey phase. Measurements of discriminant validity would remain an area to be monitored during the subsequent stages when a larger data set and more sensitive statistical tools could be employed.

expected to improve their loading. The impact of the rewording on construct loading was verified during the final data analysis stage. 


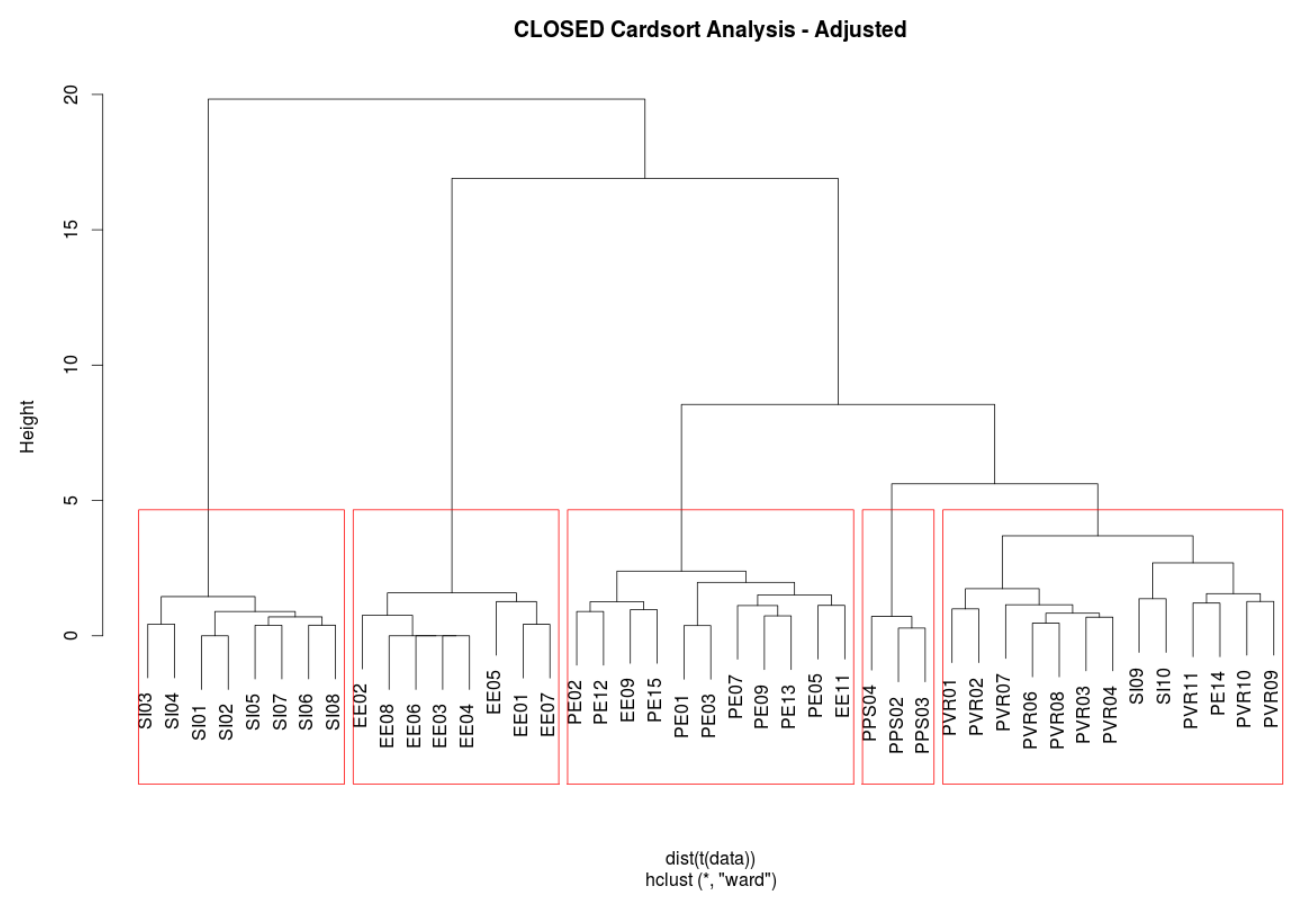

Figure 5.3: Dendrogram of the adjusted closed card sort data

\section{Open Card Sort Analysis and Results}

The open card sort data were analyzed in a similar manner to the closed card sort data, except for the creation of the agreement frequency matrix. It was not possible to create an agreement frequency matrix of the measurements as all of the categories provided by the participants were unique to each participant and ranged from 5-8 categories.

Given the lack of consistent categories, it could be expected that this data might not cluster as well as the closed card sort data. Once again, the cluster analysis dendrogram proved to be the primary analysis tool due to its ability to cluster the measurement items together independently of the number or title of the categories provided by the participants.

The initial dendrogram of the unadjusted (raw) open card sort data - see Figure 5.4 - supported the trends previously seen in the closed card sort data as shown in both Figure 5.2 on p. 118 and Figure 5.3 on p. 124; namely, that the construct categories display measurable convergent and discriminant validity. 


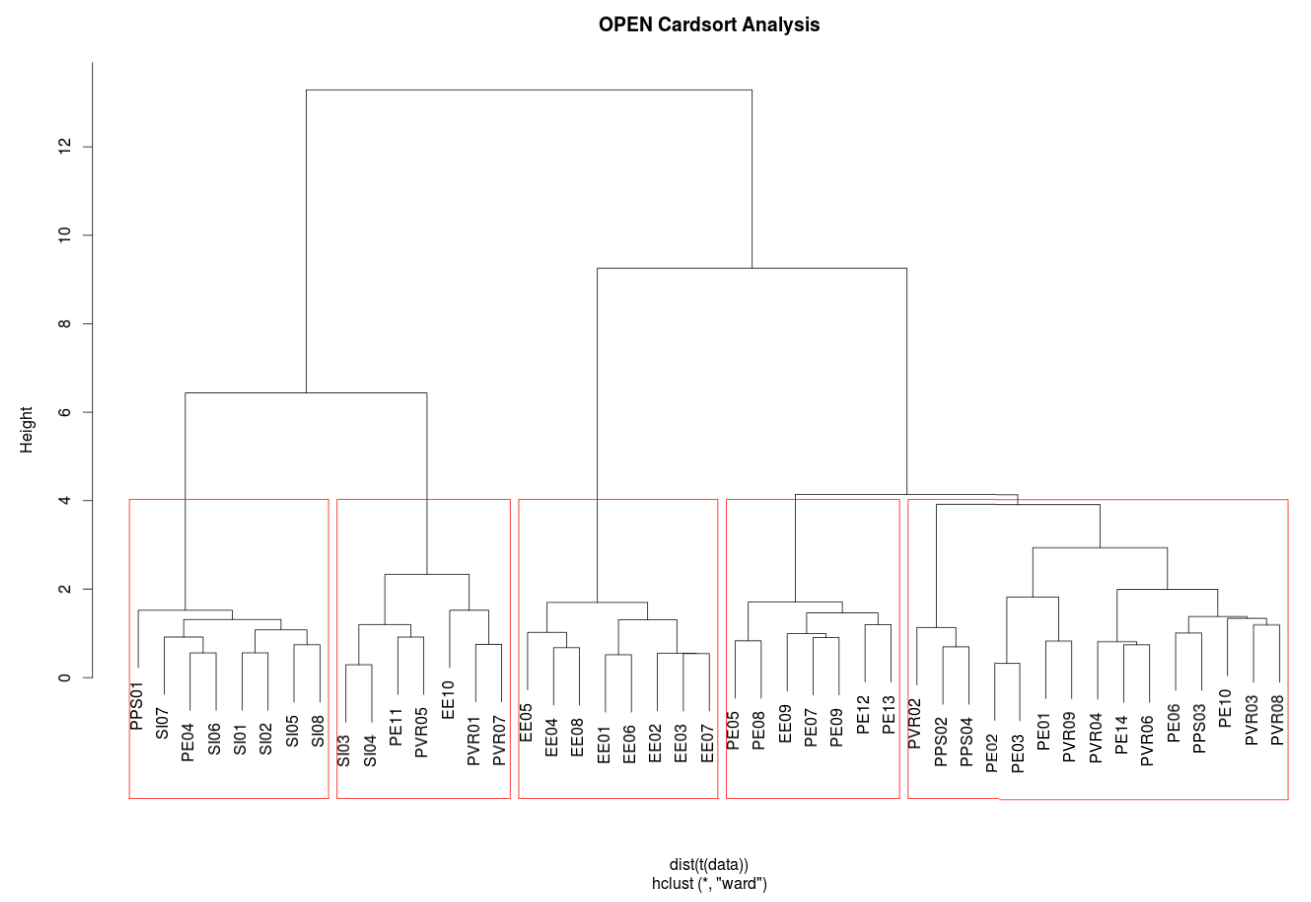

Figure 5.4: Dendrogram of the unadjusted (raw) open card sort data

On the left of Figure 5.4 are the cluster of SI items (with the exception of PPS01 and PE04). However, in the closed card sort analysis, it was noted that PPS01 and PE04 had loaded more heavily on to SI (with 0.50 and 0.58 respectively). The observation of PPS01 and PE04 being grouped with the SI measurement in the open card sort data analysis provided further confirmation of their association with the social influence construct.

Because of the observed relationship between the open and closed card sort data, as well as the general confirmation in the pattern between the two card sorts, the adjustments to the measurement items that were made based on the closed card sort agreement frequency matrix were applied to the open card sort data; namely, the removal of three measurement items and the recategorization of five other measurement items based on the closed card sort agreement frequency analysis (Table 5.18, p. 119).

The resulting adjusted open card sort dendrogram is seen in Figure 5.5. The results of this analysis confirms the closed card sort's agreement frequency matrix's findings concerning the strength of particular SI and EE measurement items. It also confirms some potential measurement-item-to-construct validity 
issues for PE and PVR, which were revisited during the survey data analysis stage.

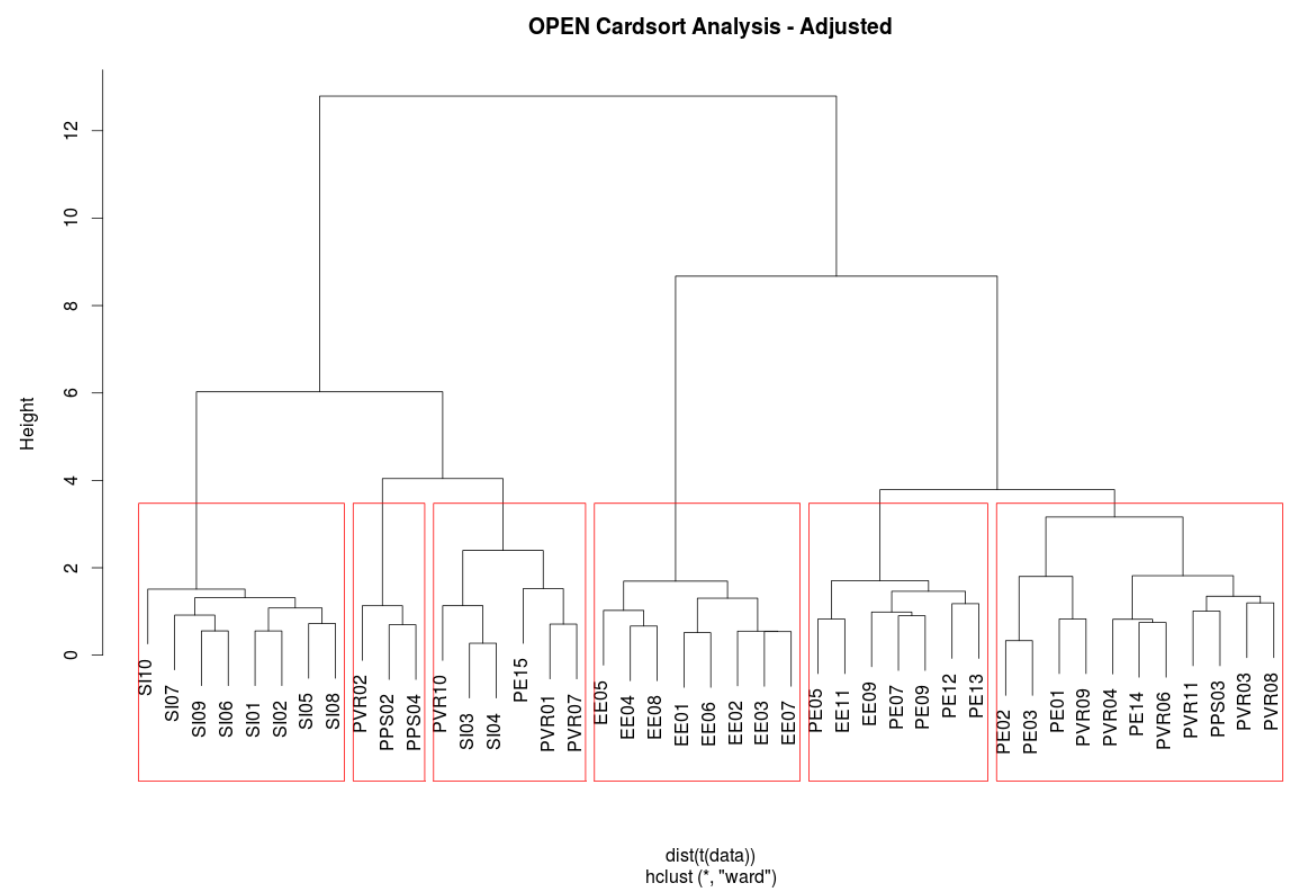

Figure 5.5: Dendrogram of the adjusted open card sort data

\subsection{Chapter Summary}

As discussed in Chapter 4, Phase I of the empirical research focused on qualitative methods with the goal of developing a set of measurement items in three stages.

The first stage was exploratory in nature. Using interviews, this stage consisted of collecting data that focused on different aspects of electronic recordkeeping system use and attitudes to and about electronic recordkeeping. Through thematic analysis, the transcripts were iteratively coded and consolidated until a number of themes emerged. These themes were then mapped to the research model. The nominated research model constructs appeared to be both necessary and sufficient to reflect the themes that emerged from the interviews. 
Efforts were then made to convert the themes to measurement items. Measurement items from the literature were first mapped to the emergent themes, as there was a strong preference to use validated measurement items where possible. Where a theme did not have a corresponding validated measurement item, one was created. Individual measurement items were then tentatively mapped to reflect their construct by the researcher.

The second stage took the additional step of proofreading the initial pool of measurement items using another ten participants. The value of this step was considerable, with over 90 issues raised (and addressed) in total. The refined set of measurement items formed the basis for stage 3's verification of the measurement item to construct relationship.

The third stage tested the proposed measurement item to construct relationship through the use of open and closed card sorting. The analysis demonstrated that the assumptions of the researcher were not necessarily shared by the participants. The card sort data highlighted several measurement items that either mapped poorly or mapped more strongly to different constructs. These were considered on a case-by-case basis. The measurement items were then remapped and the analysis was performed again, demonstrating an increase in construct validity.

Equipped with a set of qualitatively developed and validated measurement items, the focus now turns to the development of the survey instrument in the next chapter: Phase II Quantitative Methods. 


\section{Chapter 6}

\section{Phase II: Quantitative Methods}

\section{Contents}

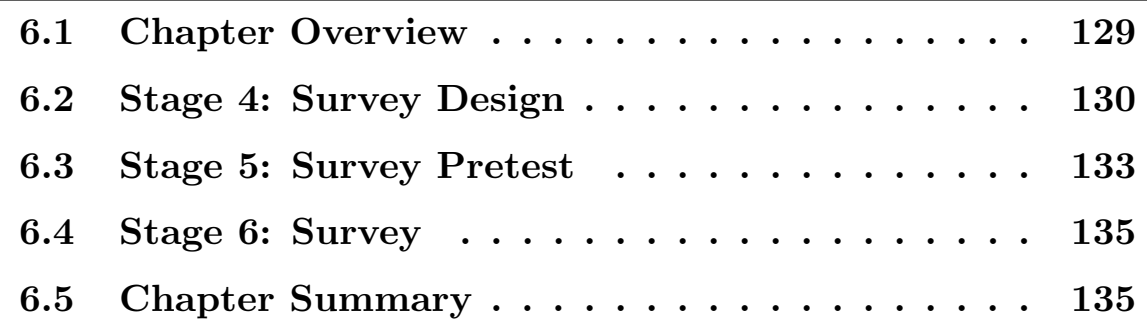

\subsection{Chapter Overview}

As noted in Chapter 4: Research Design and Methodology, Phase II of the research employed a quantitative methodology. Specifically, this phase used quantitative methods to validate the links between the measurement items and their constructs, and by capitalizing on those linkages, sought to quantitatively test the research model.

Building on Stages 1-3 of the qualitative phase, the research design of the quantitative phase consisted of three additional stages (Stages 4-6):

1. Stage 4: Survey Design: the design of the survey instrument and its implementation within a suitable online survey technology;

2. Stage 5: Survey Pretest: conducting a pretest of the online survey and using the feedback to further refine the survey instrument; and 
3. Stage 6: Survey: this was the main data-gathering exercise, which consisted of using the survey instrument to gather sufficient data to validate the underlying research model.

In the next three sections, the methods used in each is discussed in detail. Stages $4-5$ were necessarily completed prior to Stage 6 , and thus their methods included some analysis and implications that were considered before moving on to the final survey. The analysis of the Stage 6 data will be further discussed in Chapter 7: Phase II Data Analysis.

\subsection{Stage 4: Survey Design}

The survey design stage consisted of transforming the final measurement item pools that emerged from Stage 3 into an initial (draft) survey instrument. The objectives for this stage included:

1. the selection of an appropriate online survey technology;

2. consideration of the data analysis approach and the implications for how and in what detail the data needed to be captured;

3. the creation of the survey instrument, including design, item grouping, section flow, branding, etc.; and

4. the creation of associated documentation and communications templates, including participant information sheets, consent forms, introductory emails, graduated automated reminder messages, and thank you emails.

The outcome of this stage was a draft survey instrument package that included all materials required to initiate the final data-gathering activity. The survey design decisions are briefly discussed below:

\subsubsection{Online Survey Platform}

The targeted sample population (as discussed in Section 4.3) consisted of knowledge workers who had ready access to electronic recordkeeping systems as part of their work. An online survey tool was selected as the best way to reach this 'connected' audience.

The advantages of online survey tools are numerous and well-documented. They are time efficient, cost effective, customizable, capable of providing 
automated or immediate follow-up, unrestricted in geographic coverage, and able to minimize/eliminate processing and coding errors (T.-Z. Chang, 2013). An additional benefit is that they allow for direct access to public sector employees who are literally behind locked doors.

However, online surveys are not without their disadvantages. T.-Z. Chang (2013) points out that online surveys are constrained by Internet accessibility and the state of the participant's computer (e.g., featuring an up-to-date browser). Some populations are inaccessible to online surveys due to these technological constraints. Furthermore, online surveys can be overused or automated by commercial providers, leading to selection bias, truthfulness concerns (if anonymous), and even privacy concerns (T.-Z. Chang, 2013). In this research, the target population was known to be accessible online and was known to be operating compliant technology. Selection bias and privacy concerns were mitigated through the sampling procedures, information sheets, and privacy waivers. Furthermore, each invitation was customized to include the participant's name, which was expected to address the truthfulness concern associated with anonymity.

To this end, Qualtrics (http://qualtrics.com) was adopted as the survey technology platform for use in the implementation of the pretest and final survey stages. The next section addresses the survey design and data capture decisions.

\subsubsection{Analysis-Informed Data Capture}

As discussed in Section 4.4 .2 (p. 76), the selected analysis approach was a form of structural equation modeling (Partial Least Squares Path Modeling - PLSPM - see Section 7.4 on p. 162). Structural equation modeling is particularly suited for use in "inferential data analysis and hypothesis testing where the pattern of inter-relationships among the study constructs are specified a priori and grounded in established theory" (Hoe, 2008, p. 76). PLS-PM is a good fit to analyze models such as this confirmatory research model.

Knowing that the data would be analyzed using the PLS-PM technique, the survey design had to reflect that technique's requirements and capture data in a format suitable for subsequent analysis. In the case of PLS-PM, the data needed to be captured in the form of a multi-point Likert scale. A five-point Likert scale had been found to be sufficient as a measure of reliability (Hinkin, 1998) and was therefore used in the design of the survey instrument. 
Qualtrics provided standardized preset scale labels as an option during the survey design. These labels (e.g., common Likert scales such as Strongly Disagree to Strongly Agree) were accepted and incorporated into the final design. The final survey is reproduced in Appendix C on p. 239. Having selected the survey tool and data capture method, the next decision point concerned the organization of the survey and the presentation of the measurement items.

\subsubsection{Creation of Survey Instrument}

There was a final pool of 56 measurement items plus additional demographic questions. Given the need to organize these items onto short survey screens/ pages, the next question concerned how best to present them. To that end, three separate survey instruments were created using different methods of organization.

- Randomized: this survey consisted of $\approx 15$ measurement items per question block (which formed a page of the survey). The order of the questions generally followed the standard practice of starting with easier questions, which then lead on to the more thoughtful or difficult questions; however, in terms of their affiliation with specific model constructs, they were otherwise mixed together.

- Grouped by Conceptual Area: this survey consisted of three main blocks that corresponded to: the recordkeeping system, the impacts of the organizational context, and the personal opinions toward recordkeeping. The measurement items were roughly divided into the three blocks, but were otherwise presented in a random order within each block.

- Grouped by Construct: this version consisted of blocks that corresponded directly to the research model's constructs, where each block consisted of that construct's pool of measurement items $(\approx 10$ per block).

The three surveys were then considered from the viewpoint of potential participants. The survey based on the conceptual area was the first to be discarded. Although many of the measurement items fitted logically into those three groupings, several did not place well. In addition, each block consisted of as many as 20 measurement items, and this was likely to make 
the survey feel long and daunting to participants (which could negatively impact participation rates).

The randomized survey was the next to be discarded. Although there is much to be said for randomizing the measurement items, there was a risk that how a participant may interpret an item could be impacted by its contextual position next to other items. A truly randomized survey (and Qualtrics provides a randomization feature so that each participant could receive the items in a different random order) could also cause problems in comprehension and interpretation.

Thus, the survey design that grouped the measurement items by construct was selected as the preferred survey design. This design provided an opportunity to describe each construct to the participant and then introduce that construct's measurement item pool for consideration. By considering each pool individually, the participant would be able to focus on the construct dimensions portrayed by each of the measurement items in relation to each other, and this was expected to assist in how participants would interpret each measurement item, leading to improved data and model measurements.

\subsubsection{Survey Documentation Templates}

The next task was to assemble and design the associated documentation and communication templates. This included introductory emails, information sheets, consent forms, encouraging reminder email templates, and thank you templates. The final versions of these documents are shown in Appendix C and Appendix D starting on p. 239.

Having selected a survey design and drafted the survey communication templates, the survey was ready for pretesting.

\subsection{Stage 5: Survey Pretest}

The Survey Pretest is the phase where all of the parts come together for advanced testing; in other words, a 'dress rehearsal' for the final survey. Although a pretest is generally recommended, Dillman (2007) warns that the Survey Pretest is often done "haphazardly, if at all." He further emphasizes that a properly conceived pretest phase is a chance to:

- evaluate the distribution procedures and see whether any problems come up; 
- discover production mistakes by having a few people fill out the survey;

- learn whether people clearly understand all of the questions; and

- provide an opportunity for feedback to improve the survey.

In the case of an online survey, it is where the technology meets the measurement items; and a pretest can provide the researcher with an opportunity to fine-tune the survey instrument as well as the platform on which it will be administered.

Malhotra and Grover (1998) emphasized that "careful pretesting of instruments $[. .$.$] can serve as a reality check indicating to the researcher how$ well conceptualizations of the problem match the actual experience of the practitioner." Foddy (1998) also argued that "standard advice" to survey researchers has been to "field test questionnaires and interview schedules on small subsamples of respondents that have been drawn from the populations of interest so that problematic questions can be identified."

Hunt, Sparkman Jr., and Wilcox (1982) provided an early framework for identifying the role of the pretest in improving the survey instrument itself. They separated the pretest items into three categories: items about the questionnaire itself; items about specific questions; and items about data analysis (Hunt et al., 1982). Adapting this framework, the pretest of this survey instrument focused on two primary aspects of the online survey: the testing of the survey instrument itself and the testing of the survey instrument delivery, including distribution platform, mode of transmission, and ease of data file collection and processing.

The draft survey instrument was sent to a convenience sample of a dozen participants drawn from the New Zealand public sector and Victoria University of Wellington. Each of the participants was asked to complete the survey and provide feedback on the survey instrument and its individual measurement items.

The participant feedback was positive and didn't result in any changes to wording. All templates worked and the data was successfully extracted and run through a trial PLS-PM analysis. 


\subsection{Stage 6: Survey}

In November 2013, the introductory email template was sent to a panel of 254 employees of a large New Zealand public sector organization.

Appendix D (p. 249) shows the final Qualtrics communication templates and the orchestration of the timings of their release to participants. Where possible, the number of recipients for each communication template is indicated. The communication template they received was determined by their individual survey completion status at the time of each mail-out. In total, the survey was open for 13 business days.

The progress of the survey was monitored closely using the Qualtrics online monitoring tools. In addition, a personal email and telephone number was provided to all recipients to provide an easy channel for contacting the researcher should they have any questions, issues or comments.

A detailed descriptive summary of the collected survey data is provided in Appendix E (p. 257). The subsequent analysis of the collected data is covered in-depth in Chapter 7 - Phase II: Data Analysis.

\subsection{Chapter Summary}

As discussed in Chapter 4, Phase II of the research focused on quantitative methods and the goal of developing the measurement items into a survey instrument and collecting sufficient data to empirically measure the research model. Phase II included the next three stages (Stages 4-6) of the research methodology.

The first quantitative stage (Stage 4: Survey Design) covered the steps taken to design a survey instrument and select a suitable platform to orchestrate its delivery. After selecting the online survey platform, the type and format of the data needed to meet the analysis requirements was determined, resulting in the selection of a standard 5-point Likert scale. With these design decisions in place, the organization of the survey instrument was next considered. Three surveys were eventually created, and after examination of their potential advantages and disadvantages, one was selected for use as the final survey. In addition, a variety of communication templates were drafted in accordance with the modified Tailored Design Method (Dillman, 2009).

In the next stage (Stage 5: Survey Pretest), two aspects of the draft survey instrument were tested. The first aspect was the survey instrument itself, where a dozen participants were asked to fill out the survey and provide 
feedback. The second aspect tested the automated follow-up email functionality of the Qualtrics survey platform. This included several scenarios that validated the mail-merge capability as well as the tracking of the completion status. For example, the completion status determined the set of people who would receive reminder emails as opposed to thank you emails. The final pretest consisted of extracting the dozen pretest records and running them through a trial PLS-PM analysis in order to validate that the data was collected in sufficient detail and in the correct format to facilitate analysis.

The final stage covered in this chapter was Stage 6: Survey. Having developed confidence in the survey instrument and the delivery platform, the survey was sent to 254 employees of a large New Zealand public sector organization.

In the next chapter (Chapter 7 - Phase II: Data Analysis), the analysis of the survey data is discussed. 


\section{Chapter 7}

\section{Phase II: Data Analysis}

Contents

7.1 Chapter Overview . . . . . . . . . 137

7.2 Descriptive Statistics . . . . . . . . . 138

7.3 Factor Analysis . . . . . . . . . . . . . 143

7.4 Partial Least Squares Path Modeling (PLS-PM) 162

7.5 Analysis of Hypothesized Effects . . . . . . 181

7.6 Additional Comments by Respondents . . . . 184

7.7 Chapter Summary . . . . . . . . . . . 188

\subsection{Chapter Overview}

In this chapter, the analysis of the survey data is discussed. The chapter starts with a summary of the response rate and descriptive statistics associated with the sample. This is followed by a closer look at the relationship between the measurement items and their parent constructs, using a factor analysis technique in an effort to further refine and improve the measurement item pools. With the final measurement item pools selected, the preferred analysis technique, Partial Least Squares Path Modeling (PLS-PM), is introduced and its various merits discussed.

A description of the PLS-PM results for the survey data follows, with the focus first on the outer (measurement) model, and then on the inner 
(structural) model, with the estimated parameters, significance levels, and model validation analysis covered.

These results are then related back to the original research hypotheses in order to determine how well the research model approximates the observed behavior of the target population. Lastly, the qualitative data collected from an open-ended question are analyzed.

\subsection{Descriptive Statistics}

As discussed in Section 4.4.2 on estimated sample size (p. 78), the sample required for a statistically significant analysis was conservatively estimated to be between $60-120$ participants. By using PLS-PM as the primary method for analysis (see Section 7.4 on p. 162), it is likely that significant results could be obtained at the lower end of that estimate. However, non-response (and its associated bias) always remains a significant risk. As such, a generous sample size was set at $\approx 200$ participants and an effort was made to minimize non-response as much as possible.

\subsubsection{Response Rate}

The survey consisted of 254 invitations that were emailed out to a large New Zealand public sector organization consisting of a cross section of business units and responsibilities. ${ }^{1}$ The personal assistant to one of the general managers volunteered to compile a list of suitable employees, which was imported as a panel within Qualtrics. Each record included the first name, last name, email address, title, and phone number, which assisted greatly in personalizing the mass emailed invitations.

Of the 254 invitations, 208 surveys were opened and viewed, resulting in an $81.89 \%$ opening rate. Of the 208 opened surveys, there were 193 usable records, consisting of:

\footnotetext{
${ }^{1}$ Although the sample was limited to one organization, it captured a cross-section of functions and activities that was expected to provide a similar representative variability to that of the public sector study population. It has been observed that "the relative importance of business unit effects far outweighs those of corporate or industry effects" (Misangyi, Elms, Greckhamer, \& Lepine, 2006, p. 587), and it is argued that the differences in attitudes between functional business units is likely greater than the aggregate differences between public sector organizations.
} 
- 101 responses (48.6\% of 208) that were $100 \%$ complete (representing $39.76 \%$ of all invitations);

- 91 responses ( $43.8 \%$ of 208 ) that were $>90 \%$ complete (e.g., completed all measurement items, but did not answer all of the optional demographic questions such as 'age' or provide text in the optional comment box); and

- 1 response $(0.5 \%$ of 208$)$ that was $>80 \%$ complete - i.e., answered all of the measurement item questions, but none of the demographic questions.

This means that of the 208 opened invitations, $92.8 \%$ provided usable data, or a $76.0 \%$ usable response rate against all invitations. This relatively high response rate avoids the majority of the risk associated with non-response bias (Sivo et al., 2006). Of the remaining 15 opened surveys:

- 10 responses (4.8\% of 208) did not agree to the terms of the survey.

- 5 responses completed less than $30 \%$ of the questions and thus were excluded from the study.

The 193 participant responses were exported to a text file and used for the subsequent analysis.

\subsubsection{Sample Demographics}

Several different demographic measures of the sample were collected, including age, gender, indication of seniority or influence, and the duration of their employment with the target organization.

Some observations from the collected demographic data are listed below.

- Age (Figure 7.1a): the age profile was unremarkable, with a median age of approximately 45 years.

- Gender (Figure 7.1b): the gender was skewed to female.

- Seniority (Figure 7.1c): the sample population consists of a mix of workers and team leaders and/or managers, representing a ratio of 4:1.

- Employment duration (Figure 7.1d): the employment duration profile indicates a stable group of workers who could be expected to be familiar with organizational policies and procedures, with $71 \%$ having more than 2 years of experience working in the organization. 


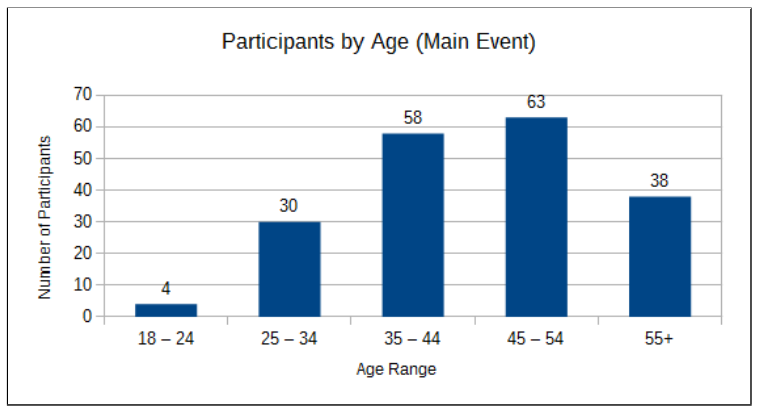

(a) Age

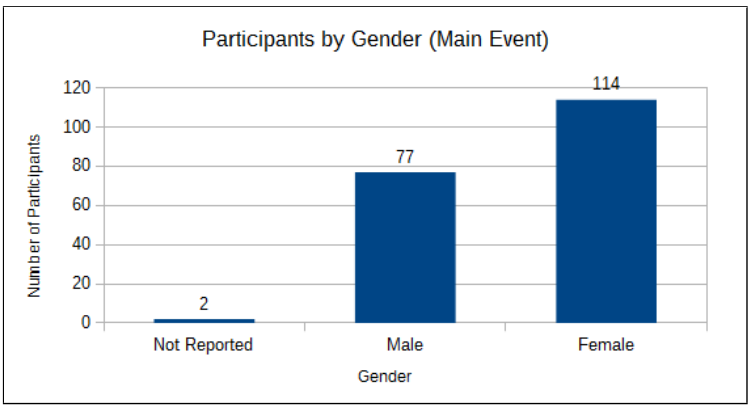

(b) Gender

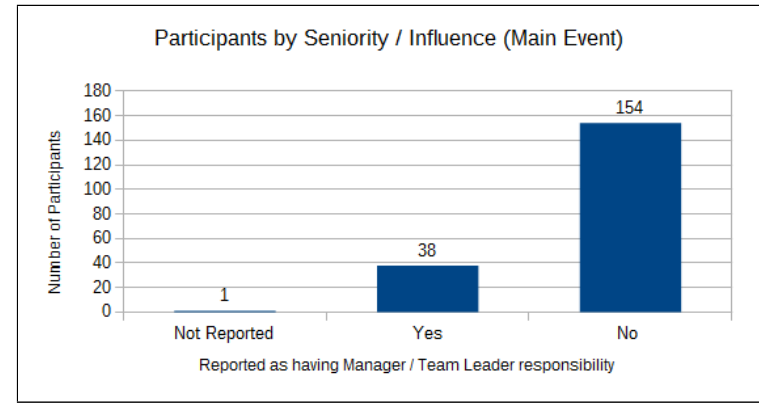

(c) Position of Seniority or Influence

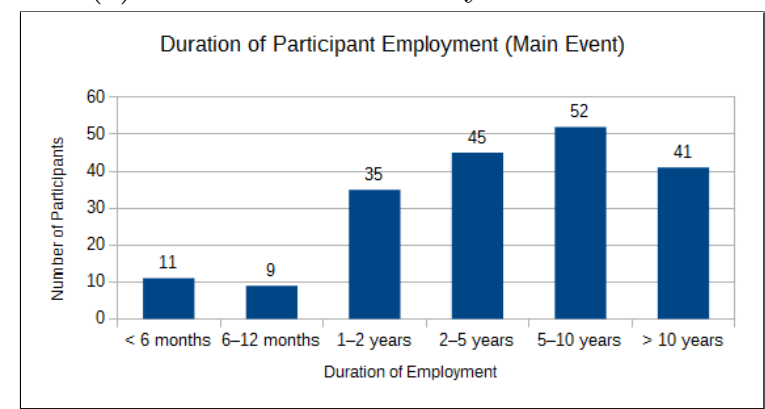

(d) Employment Duration

Figure 7.1: Demographics of the survey participants, including: (a) age, (b) gender, (c) position of seniority or influence, and (d) employment duration. 


\subsubsection{EDRMS Experience Profile}

During the preparations for the survey, the targeted public sector organization reported that their electronic recordkeeping system had not yet been made available to all business units. Some business units had access to the system for years, whereas others had only been using the system for a few months (and some not at all - these were not included in the sample). In order to account for the experience of the participants (or their stage of 'continued use' - see Figure 2.5, p. 31) and their knowledge of their organization's records policies and guidelines, three questions were asked. Figure 7.2 illustrates the distribution of the target sample's responses.

- Frequency of use (Figure 7.2a): although the electronic recordkeeping system had been made available to all staff in the sample population, only $56.4 \%$ used it daily. However, $88.0 \%$ used it at least once a month, which was expected to be sufficient to maintain their skills.

- Duration of experience (Figure 7.2b): the majority of the participants had significant experience with the electronic recordkeeping system, with $84.4 \%$ having at least a full year of usage experience.

- Awareness of records policy (Figure 7.2c): in spite of the Public Records Act and the EDRMS training received, when asked whether recordkeeping was mandatory, $40 \%$ either responded no or not sure.

The above descriptive statistics are provided in order to better describe the sample population and to provide additional context for the research model findings. However, the impact that any of the sample population demographics (e.g., gender or age) may have had on the outcome of the research model is out of scope.

In the next section, the measurement items that make up the bulk of the survey are considered in light of their associated construct groupings. 


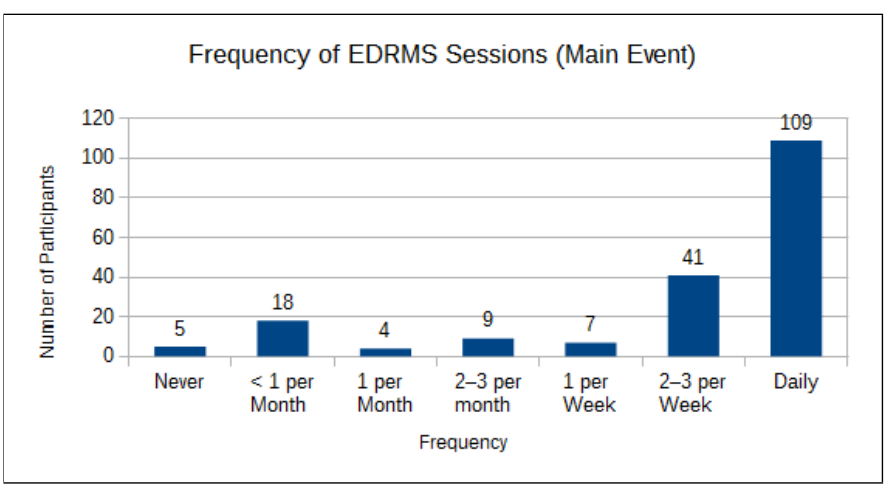

(a) Frequency of Use

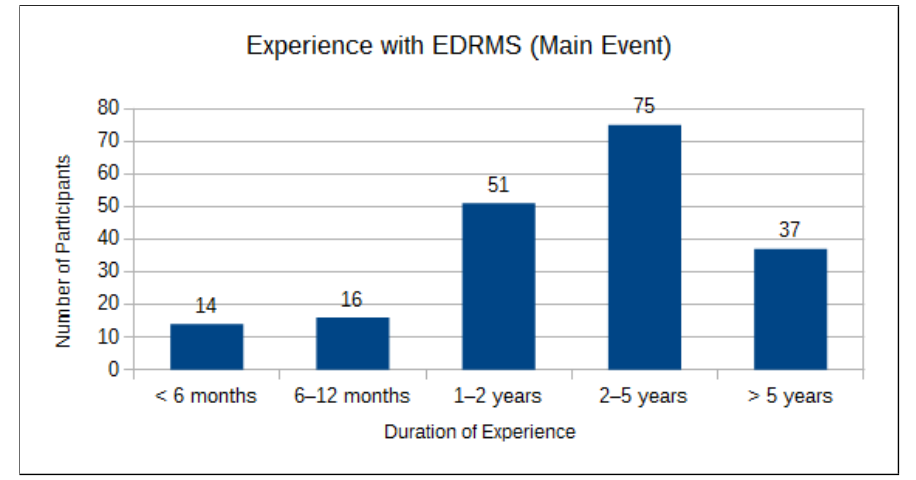

(b) Duration of EDRMS Experience

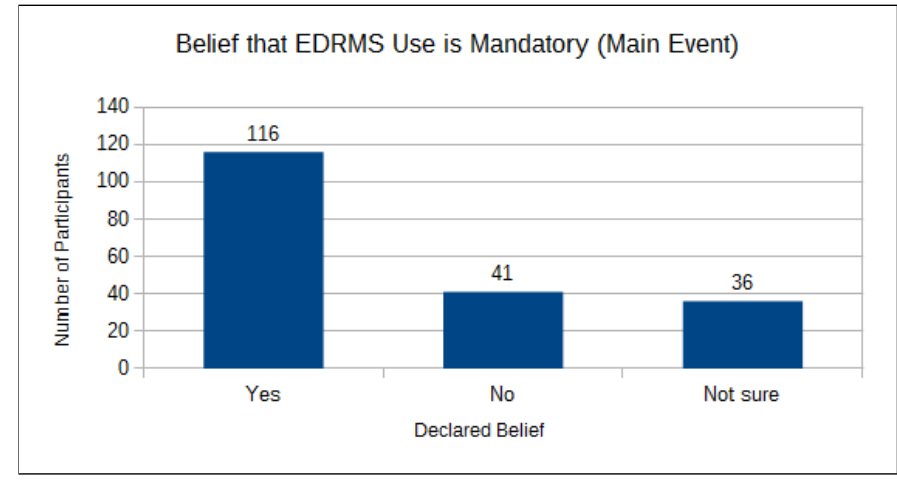

(c) Recordkeeping is Mandatory

Figure 7.2: Profile of the sample population's experience with their EDRMS, including: (a) frequency of use, (b) duration of EDRMS experience, and (c) belief that recordkeeping is mandatory. 


\subsection{Factor Analysis}

The initial selection process was based on Principal Component Analysis (PCA). This statistical method was used to measure how well each of the measurement item pools co-correlated with each other.

The goals of PCA are to (a) extract the most important information from the data table, (b) compress the size of the data set by keeping only this important information, (c) simplify the description of the data set, and (d) analyze the structure of the observations and the variables. (Abdi \& L. Williams, 2010, p. 3)

To achieve these goals, PCA computes a new set of variables (principal components) that represent a linear combination of the original variables. The first principal component is the one that explains the largest source of inertia, where inertia is a term used to represent the total variance of the data. The second principal component is computed "under the constraint of being orthogonal to the first component and to have the [next] largest possible inertia" (Abdi \& L. Williams, 2010, p. 4).

If one were to imagine a positive correlation scatter plot (with the points forming a diagonal distribution from the lower left to the upper right of the graph), the first principal component would describe the primary regression line moving in the same direction (in parallel) with the correlation distribution and would thus describe the majority of the observed variance (inertia). The second principal component would describe the width of the distribution and is measured as being perpendicular (orthogonal) to the first principal component. The first two principal component variables thus capture the majority of the inertia associated with a regular positive correlation scatter plot. These variables are called, depending on their context, either "principal components, factors, eigenvectors, singular vectors, or loadings" (Abdi, 2003, p. 1).

If the correlation scatter plot were irregularly shaped, additional principal components could be similarly computed (and may be required to explain the additional inertia/variance). Principal component calculations result in factor scores that can be "interpreted geometrically as the projections of the observations onto the principal components" (Abdi \& L. Williams, 2010, p. 4).

The circle of correlations is a common visualization to help with the interpretation of the first two principal components. A high correlation on the 
first and second principal components indicates that the measurement items are also sufficiently correlated to perform well in the final PLS-PM analysis, which uses a similar statistical method for calculating its outer model.

PCA (via the circle of correlations) was applied to each of the model constructs in turn in order to verify aspects of construct validity and select a final pool of measurement items for use in the structural equation model.

\subsubsection{Circle of Correlations}

The circle of correlations consists of a plot of correlations between the original variables and a pair of principal components (usually the first two). For an example, see Figure 7.3 (p. 146). Abdi and Williams (2010, p. 11) provided a method to interpret the circle of correlations as follows:

Recall that the sum of the squared loadings for a variable is equal to one. Remember, also, that a circle is defined as the set of points with the property that the sum of their squared coordinates is equal to a constant. As a consequence, when the data are perfectly represented by only two components, the sum of the squared loadings is equal to one, and therefore, in this case, the loadings will be positioned on a circle which is called the circle of correlations. When more than two components are needed to represent the data perfectly, the variables will be positioned inside the circle of correlations. The closer a variable is to the circle of correlations, the better we can reconstruct this variable from the first two components (and the more important it is to interpret these components); the closer to the center of the plot a variable is, the less important it is for the first two components.

When plotting multiple measurement items on to the same circle, a tight cluster demonstrates that the measurement items reflect similar aspects of the same latent construct. As such, highly clustered measurement items are regarded as being better predictors of the latent construct (Sanchez, 2013b). Measurement items that did not cluster well were reviewed for possible alternative interpretations, and some were dropped from the measurement item pools.

In the next section, the analysis of each construct and how its measurement item pool performed is described, with an emphasis on how the analysis results were interpreted and actioned to create the final pool of measurement items taken forward for further structural analysis. 


\subsubsection{Factor Analysis by Construct}

The PCA-based circle of correlations, in combination with the qualitative card sorting data collected in Phase I and discussed in Chapter 5, Section 5.4 (p. 112), was used to identify the measurement items that were highly correlated and thus provided a tight unidimensional reflection of their construct. Measurement items that did not cluster well were reviewed for the possibility of different semantic interpretations and other potential sources of error. Many measurement items were subsequently removed from the final measurement model. Each of the constructs (and their related measurement item pools) are discussed below.

\section{Effort Expectancy (Ease of Use) Factor Analysis}

The first construct under consideration was effort expectancy (ease of use). Effort expectancy measurement items grouped well during the card sorting exercises (see Table 5.19 on p. 123) with several items sorting perfectly among all participants. In this stage, the larger dataset collected during the survey supported the use of the more robust PCA-based circle of correlations as a method to confirm the earlier card sorting analysis. The resulting effort expectancy (EE) circle of correlations is shown in Figure 7.3.

Figure 7.3 shows that the EE measurement items were generally clustered and shared a similar directional alignment. In addition, the majority of the items were well explained by the first two principal components (as illustrated by the proximity to the edge of the circle). Note that EE09 is closer to the center of the circle than the other items, and this indicates that at least one additional principal component may be required to fully explain the shape of its distribution. This could also mean that survey participants interpret the EE09 measurement item as having additional meanings or dimensions that are beyond the context intended - i.e., an unintended source of error.

The primary cluster of EE measurement items in Figure 7.3 is designated with the manual addition of a small red circle. All of the measurement items within that circle were considered to be well correlated and were expected to reflect the construct in the final measurement item with low error. Table 7.1 provides the full text of the EE measurement items and divides the primary cluster of measurement items from the outliers (those that did not cluster as well) to assist with the interpretation.

The initial observation was that the measurement items in the primary EE cluster coincided with the highest scoring measurement items from the 


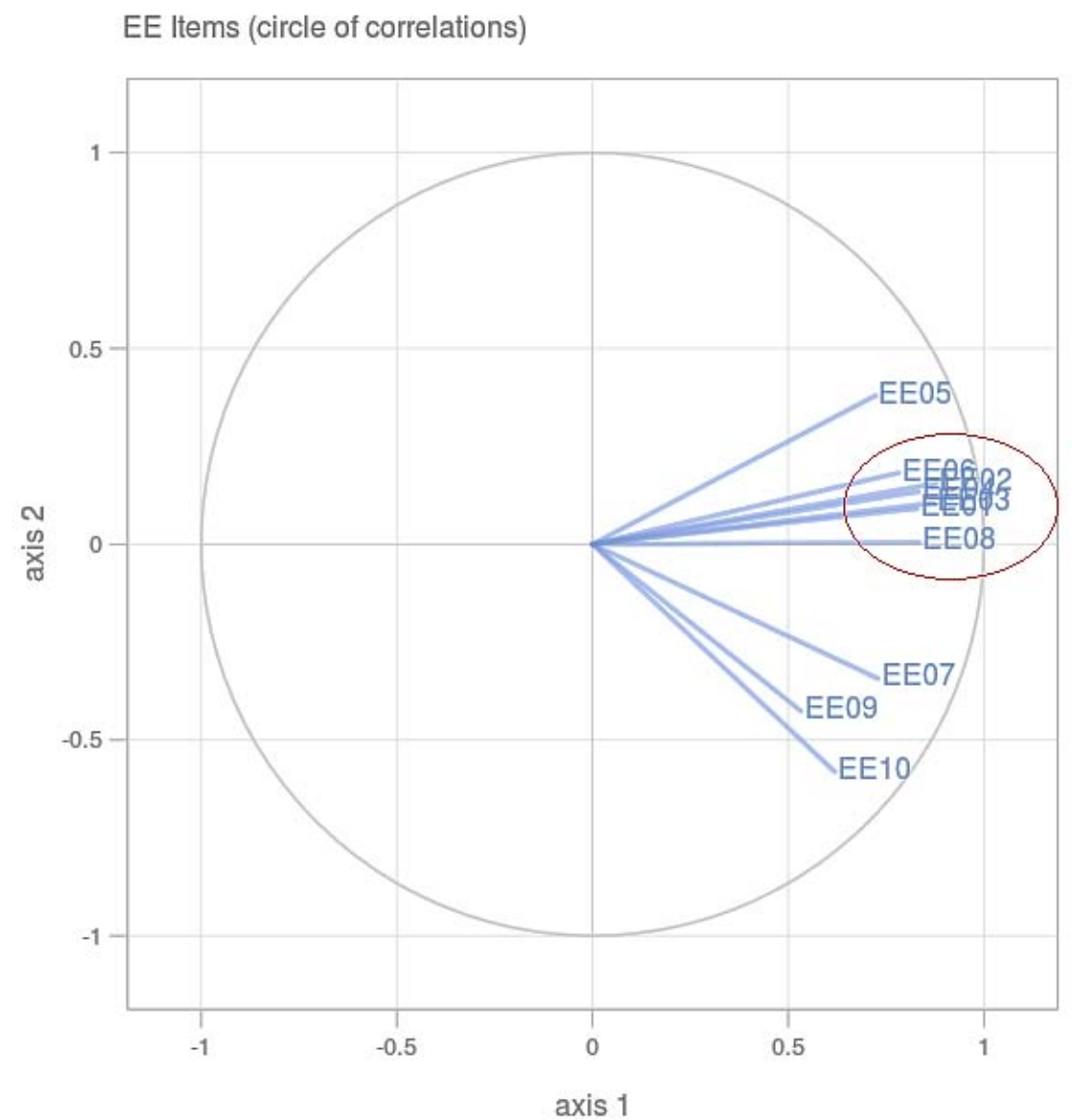

Figure 7.3: Circle of Correlations: Effort Expectancy (ease of use) measurement items. The small red circle indicates the primary cluster and suggested the need for additional consideration of the remaining measurement items. 
Effort Expectancy (EE): Principal Component-based Item Selection

\begin{tabular}{|l|l|l|}
\hline PCA Primary Cluster (Keep in Model) & Comments \\
\hline EE01 & $\begin{array}{l}\text { I find the system's interface (e.g., its } \\
\text { menus and layout) to be clear and un- } \\
\text { derstandable. }\end{array}$ & \\
\hline EE02 & $\begin{array}{l}\text { I find it easy to become skillful at using } \\
\text { the system. }\end{array}$ & $\begin{array}{l}\text { All of these measurement } \\
\text { items are indicated as being } \\
\text { part of the primary EE PCA } \\
\text { cluster. Semantically, } \\
\text { they also reflect a similar as- } \\
\text { pect of software ease of use. }\end{array}$ \\
\hline EE04 & $\begin{array}{l}\text { I find the system easy to use in my job to operate the system is easy. } \\
\text { EE06 }\end{array}$ & $\begin{array}{l}\text { The process of contributing a record } \\
\text { to the system (electronic filing) is easy } \\
\text { and straightforward. }\end{array}$ \\
\cline { 1 - 2 } EE08 & $\begin{array}{l}\text { I can learn how to do administrative } \\
\text { tasks in the system without difficulty. }\end{array}$ & \\
\hline
\end{tabular}

\begin{tabular}{|l|l|l|}
\hline PCA Outlier Items (Drop from Model) & Comments \\
\hline EE05 & $\begin{array}{l}\text { I use the system often enough to main- } \\
\text { tain my skill in its use. }\end{array}$ & $\begin{array}{l}\text { Targets skill fade rather } \\
\text { than ease-of-use. Drop from } \\
\text { item pool. }\end{array}$ \\
\hline EE07 & $\begin{array}{l}\text { I have no trouble searching for records } \\
\text { in the system. }\end{array}$ & $\begin{array}{l}\text { Searching relies on search } \\
\text { term selection skill, and less } \\
\text { on software design. Drop } \\
\text { from item pool. }\end{array}$ \\
\hline EE09 & $\begin{array}{l}\text { I can access the system directly from } \\
\text { other computer applications - e.g., di- } \\
\text { rectly from Microsoft Office. }\end{array}$ & $\begin{array}{l}\text { Measures awareness of exist- } \\
\text { ing functionality, not ease-of- } \\
\text { use. Drop from item pool. }\end{array}$ \\
\hline EE10 & $\begin{array}{l}\text { I find that the records are logically } \\
\text { organized in the system. }\end{array}$ & $\begin{array}{l}\text { Reflects the logic of the } \\
\text { records classification rather } \\
\text { than the ease-of-use of the } \\
\text { software. Drop from item } \\
\text { pool. }\end{array}$ \\
\hline
\end{tabular}

Table 7.1: Effort Expectancy (EE): Semantic analysis of the primary PCA cluster of EE measurement items, including items that were removed from the measurement item pool due to poor PCA alignment. 
card sorting frequency matrix in Table 5.19 (p. 123). This provided strong empirical support (using the larger survey data set) for the earlier qualitative observations that emerged from the card sorting activity.

The remaining four outlier measurement items were reviewed to determine if there were different possible interpretations of their meaning or if there were the potential for them to target concepts that are only indirectly associated with ease of use.

As noted in Table 7.1, EE05 reflected aspects of ease of use, but also incorporated aspects related to skill fade. EE07 targeted the dimension of search; however, it could also be interpreted to incorporate the level of a user's search skill. EE09 sought to reduce effort expectancy through integration with other office software; however, this item likely measured the awareness of existing functionality rather than its ease of use. Finally, EE10 could be interpreted as reflecting the logic of the records classification rather than the ease of use of the software interface.

Therefore, EE05, EE07, EE09, and EE10 were subsequently removed from the measurement model. The remaining measurement items - EE01, EE02, EE03, EE04, EE06 and EE08 - were retained and formed the final EE measurement item pool going forward.

As illustrated above, PCA (when considered in combination with the earlier qualitative analysis) provided a method to further select the most correlated measures of effort expectancy. In the next sections, this method was repeated for performance expectancy and the other remaining research model constructs.

\section{Performance Expectancy (Usefulness) Factor Analysis}

Performance expectancy (usefulness) was one of the poorest performing pools of measurement items during the card sorting exercise. The closed card sorting (Table 5.18, p. 119) found that the average agreement frequency for PE items was only 0.55 , with five of the original items performing poorly enough that they were either rewritten, recategorized to a different construct, or completely removed from the measurement item pool prior to their use in the survey. The best performing item in the card sorting activity (PE01) only achieved a 0.83 agreement frequency.

Figure 7.4 displays the results of the circle of correlations on the final set of PE measurement items. This analysis indicated that the improvements to the measurement items (as a result of the card sorting activity) were generally successful when implemented in the survey. Again, the manual addition of a 


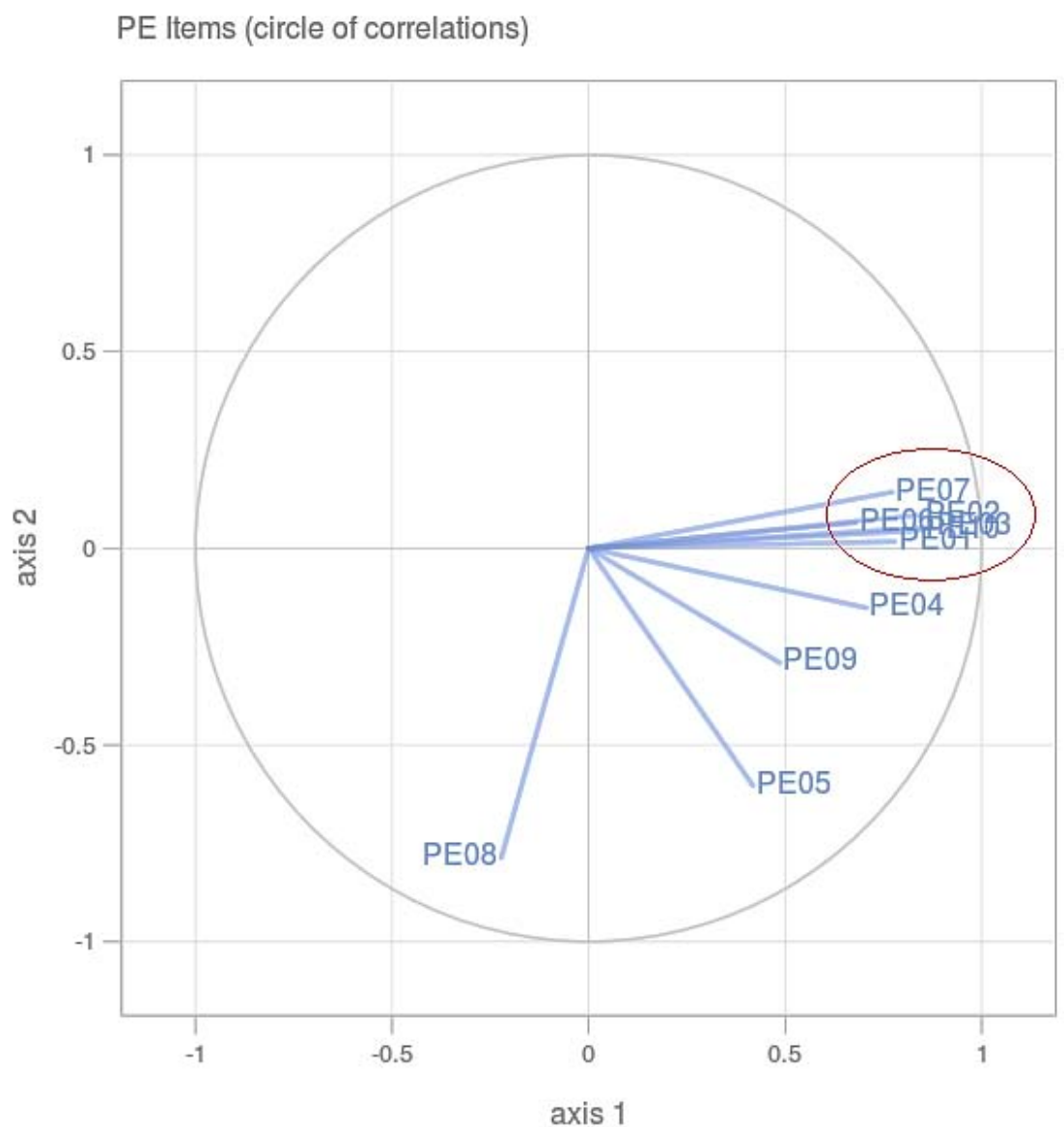

Figure 7.4: Circle of Correlations: Performance Expectancy (Usefulness) measurement items indicating the primary cluster. The small red circle indicates the primary cluster and suggested the need for additional consideration of the remaining measurement items. 
Performance Expectancy (PE): Principal Component-based Item Selection

\begin{tabular}{|c|c|c|}
\hline \multicolumn{2}{|c|}{ PCA Primary Cluster (Keep in Model) } & Comments \\
\hline PE01 & I find the system useful in my job. & \multirow{6}{*}{$\begin{array}{l}\text { All of these measurement } \\
\text { items are indicated as being } \\
\text { part of the primary PE PCA } \\
\text { cluster. Semantically, they } \\
\text { also reflect a similar aspect of } \\
\text { software usefulness. }\end{array}$} \\
\hline PE02 & $\begin{array}{l}\text { Using the system enables me to accom- } \\
\text { plish tasks more quickly. }\end{array}$ & \\
\hline PE03 & $\begin{array}{l}\text { Using the system increases my produc- } \\
\text { tivity. }\end{array}$ & \\
\hline PE06 & I have found the system to be reliable. & \\
\hline PE07 & $\begin{array}{l}\text { The system's ability to integrate with } \\
\text { my other computer applications ulti- } \\
\text { mately saves me time in my job. }\end{array}$ & \\
\hline PE10 & $\begin{array}{l}\text { The system is flexible enough to sup- } \\
\text { port both my needs as well as the or- } \\
\text { ganization's requirements. }\end{array}$ & \\
\hline
\end{tabular}

\begin{tabular}{|l|l|l|}
\hline \multicolumn{2}{|c|}{ PCA Outlier Items (Drop from Model) } & Comments \\
\hline PE04 & $\begin{array}{l}\text { Using the system's Search Function } \\
\text { allows me to locate records effectively. }\end{array}$ & $\begin{array}{l}\text { May incorporate search skill } \\
\text { in addition to usefulness. } \\
\text { Drop from item pool. }\end{array}$ \\
\hline PE05 & $\begin{array}{l}\text { The system effectively manages secu- } \\
\text { rity to the level required for my sens- } \\
\text { tive records. }\end{array}$ & $\begin{array}{l}\text { Many users may not use se- } \\
\text { curity features or connect } \\
\text { those features with usefulness. } \\
\text { Drop from item pool. }\end{array}$ \\
\hline PE08 & $\begin{array}{l}\text { The system is often slow in its opera- } \\
\text { tion. }\end{array}$ & $\begin{array}{l}\text { Speed is not necessarily cor- } \\
\text { related with usefulness. Drop } \\
\text { from item pool. }\end{array}$ \\
\hline PE09 & $\begin{array}{l}\text { I use the system to report on the status } \\
\text { of various records. }\end{array}$ & $\begin{array}{l}\text { People may have different } \\
\text { ideas of what "reporting" } \\
\text { means, or may not use that } \\
\text { feature. Drop from item pool. }\end{array}$ \\
\hline
\end{tabular}

Table 7.2: Performance Expectancy (PE): Semantic analysis of the primary PCA cluster of PE measurement items, including items that were removed from the measurement item pool due to poor PCA alignment. 
small red circle indicates the primary cluster of measurement items near the outer edge of the circle.

Unfortunately, Figure 7.4 also shows four outlier measurement items that were not clustered and were also well within the circle boundary, indicating that they do not share the same principal components as the primary cluster. The location in the circle of correlations suggests that they may contain additional dimensions or methods of interpretation by participants.

Table 7.2 provides the full text of the PE measurement items and divides the primary cluster from the outliers. Reviewing the remaining four outlier measurement items showed that there was either room for different interpretations of their meaning or the potential for them to target concepts that are only indirectly associated with usefulness.

PE04 sought to categorize search as useful; however, the measurement item could also be interpreted as incorporating aspects of a user's search skill. PE05 sought to determine the usefulness of managing security; however, many users may not be aware of that capability or may not consider it as part of software usefulness. PE08 sought to link speed to usefulness; however, speed is not necessarily correlated with usefulness. Note that PE08 shared the least directional orientation in the circle of correlations. Finally, PE09 sought to include reporting as a dimension of usefulness; however, people may have differing ideas of what reporting entails or may not use the reporting function.

Therefore, PE04, PE05, PE08, and PE09 were removed from the measurement model. The remaining measurement items - PE01, PE02, PE03, PE06, PE07 and PE10 - were retained as the PE measurement item pool for later use in the final structural model.

\section{Social Influence and Perceived Power Security Factor Analysis}

The social influence (SI) measurement items were one of the higher performing construct pools in the card sorting activities. For an example, see Figure 5.3 on p. 124. However, when subjected to the more sensitive principal component analysis and graphed in the circle of correlations, three distinct clusters emerged from the SI measurement item pool. This is illustrated in Figure 7.5 below.

A theoretical basis to help explain this clustering was sought from the literature. The original selection of the organizational context constructs, following a review of the relevant literature (Section 2.5.2, p. 45), was based on 
Social Influence items (circle of correlations)

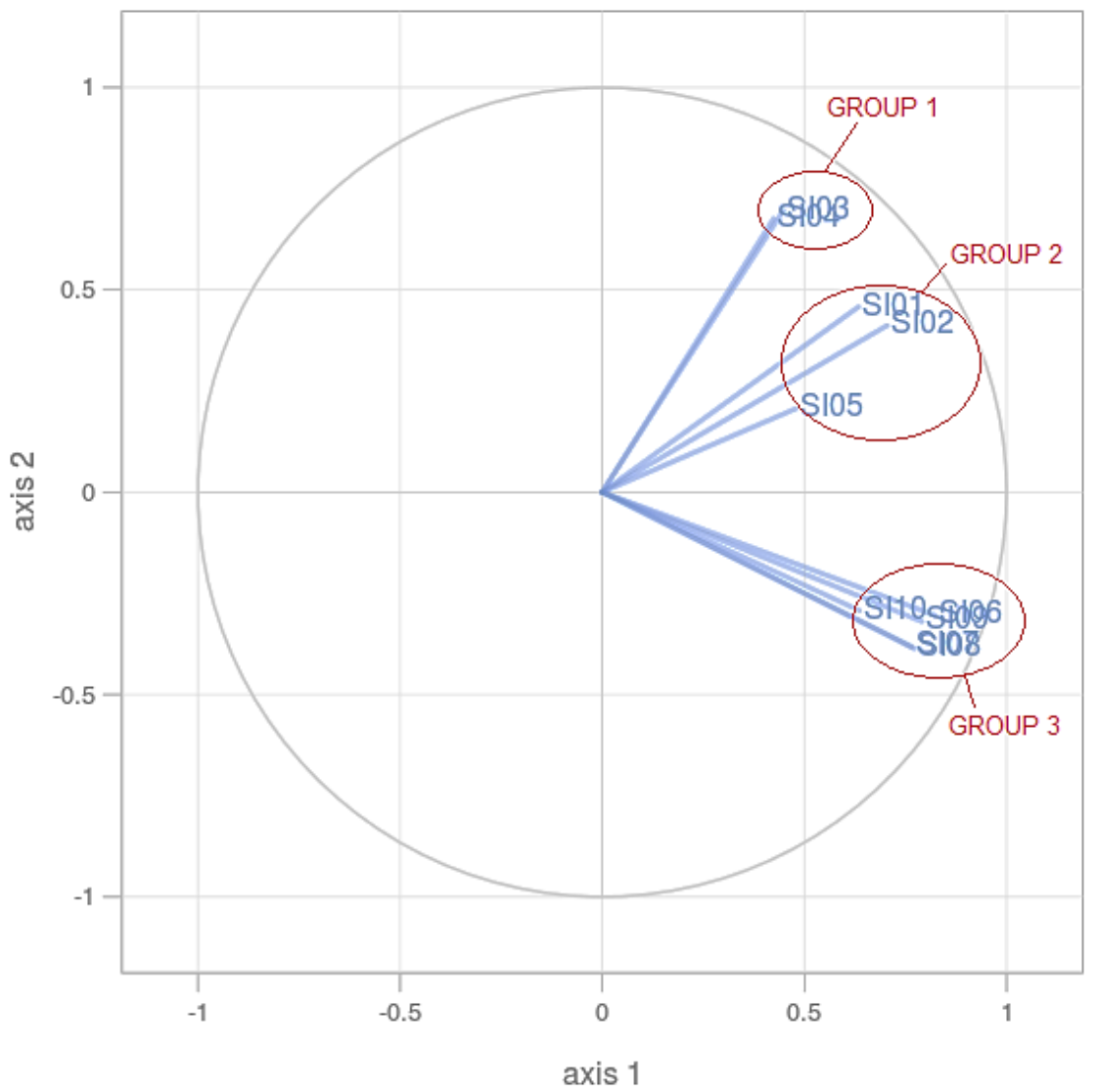

Figure 7.5: Circle of Correlations: Social Influence measurement items. The items formed three clusters, indicated by Groups 1-3. 
Social Influence(SI): Principal Component-based Item Selection

\begin{tabular}{|l|l|l|}
\hline \multicolumn{2}{|l|}{ PCA Group 1 } & Comments \\
\hline SI03 & $\begin{array}{l}\text { The senior management of this organi- } \\
\text { zation support the use of the system. }\end{array}$ & $\begin{array}{l}\text { Sanction: reflects the sanc- } \\
\text { tion interaction via the struc- } \\
\text { ture of Legitimation } \\
\text { and the modality of Norm. }\end{array}$ \\
\hline SI04 & $\begin{array}{l}\text { In general, the organization supports } \\
\text { the use of the system. }\end{array}$ & \\
\hline
\end{tabular}

\begin{tabular}{|c|c|c|}
\hline \multicolumn{2}{|c|}{ PCA Group 2} & Comments \\
\hline SI01 & $\begin{array}{l}\text { People who influence my behavior think } \\
\text { that I should use the system. }\end{array}$ & \multirow{3}{*}{$\begin{array}{l}\text { Communication: uses the } \\
\text { traditional measures of Sub- } \\
\text { jective Norm and Social Influ- } \\
\text { ence to reflect Giddens' } \\
\text { communication interaction. }\end{array}$} \\
\hline SI02 & $\begin{array}{l}\text { People who are important to me think } \\
\text { that I should use the system. }\end{array}$ & \\
\hline SI05 & $\begin{array}{l}\text { I use the system because many of my } \\
\text { co-workers also use the system. }\end{array}$ & \\
\hline
\end{tabular}

\begin{tabular}{|c|c|c|}
\hline \multicolumn{2}{|c|}{ PCA Group 3} & Comments \\
\hline SI06 & $\begin{array}{l}\text { People in my organization who use the } \\
\text { system are more highly regarded than } \\
\text { those who do not. }\end{array}$ & \multirow{5}{*}{$\begin{array}{l}\text { Power: Reflects aspects of } \\
\text { Giddens' power interaction } \\
\text { by providing indications of } \\
\text { ranking and means of facil- } \\
\text { itating domination and pro- } \\
\text { moting status. }\end{array}$} \\
\hline SI07 & $\begin{array}{l}\text { People in my organization who use the } \\
\text { system are generally more dependable } \\
\text { than those who do not. }\end{array}$ & \\
\hline SI08 & $\begin{array}{l}\text { People in my organization who regularly } \\
\text { use the system acquire a higher profile. }\end{array}$ & \\
\hline SI09 & $\begin{array}{l}\text { Using the system increases my chances } \\
\text { of getting recognition in the workplace - } \\
\text { e.g., contributes to promotion chances. }\end{array}$ & \\
\hline SI10 & $\begin{array}{l}\text { Placing my documents in the system, } \\
\text { where other people may view them, may } \\
\text { positively effect my reputation. }\end{array}$ & \\
\hline
\end{tabular}

Table 7.3: Social Influence (SI): Semantic analysis of the three PCA clusters of SI measurement items. The PCA clustering aligns well with Giddens' social interactions of communication, power, and sanction. 
Giddens' Structuration Theory and his social interactions of communication, power, and sanction. (See Figure 2.2 on p. 20.)

Table 7.3 groups the SI measurement items by their PCA cluster and aligns them with Giddens' social interactions. The interpretation of this cluster analysis indicates that the social influence measurement items incorporate more than a single dimension of the construct, and that social influence can be further considered in terms of its structurational components. This interpretation is explored in Chapter 8 Discussion, p. 195.

The initial selection of social influence as a construct was made because it partially reflected Giddens' interactions of communication and to some extent sanction, with power being reflected separately by perceived power security (PPS). However, by adding in additional measurement items from component constructs that were claimed by social influence, the appearance of three clusters (including one that could be interpreted as covering power) raised a question. Were the measurement items contained in Figure 7.5 Group 3 (power) sufficiently aligned with PPS to consider merging the two power item pools into one?

Perceived Power Security Factor Analysis When applying PCA to the PPS data, the resulting circle of correlations for the PPS measurement items revealed relatively weak clustering. See Figure 7.6.

The PPS measurement items had been problematic throughout the first stages of the research. They were among the most targeted for comprehension problems during proofreading; and subsequently, they did not sort well in the card sorting activity. The survey data (see p. 261) provided what appeared to be conflicting responses - i.e., participants reported that use of the system simultaneously increased and decreased their power and influence over others. Finally, the PCA analysis confirmed only a weak relationship between the measurement items. Nonetheless, were these still power measurement items in spite of their imperfections?

With the SI measurement item pool providing a potential alternative power grouping (i.e., the Figure 7.5 Group 3 power cluster), additional analysis was conducted to determine the extent of the overlap of PPS and SI measurement items in terms of their principal component alignment.

To this end, the PPS and SI measurement items were combined into a single pool for analysis. Figure 7.7 revealed that the PPS measurement items appeared to cluster well in the context of the other SI measurement items that had been associated with power, thus seeming to create a larger 


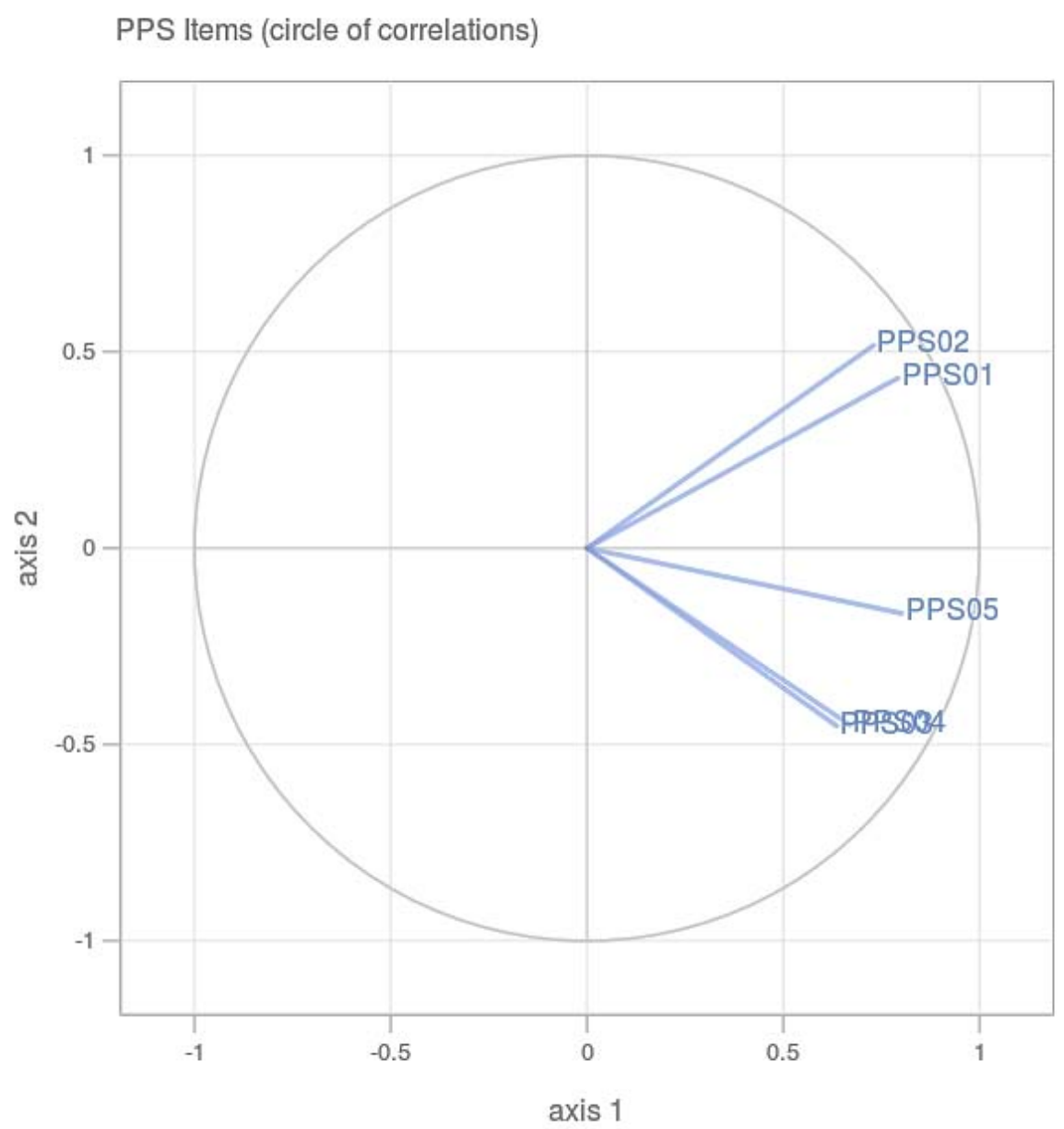

Figure 7.6: Circle of Correlations: perceived power security items reveal a lack of adequate clustering on their own. 
SI \& PPS Items (circle of correlations)

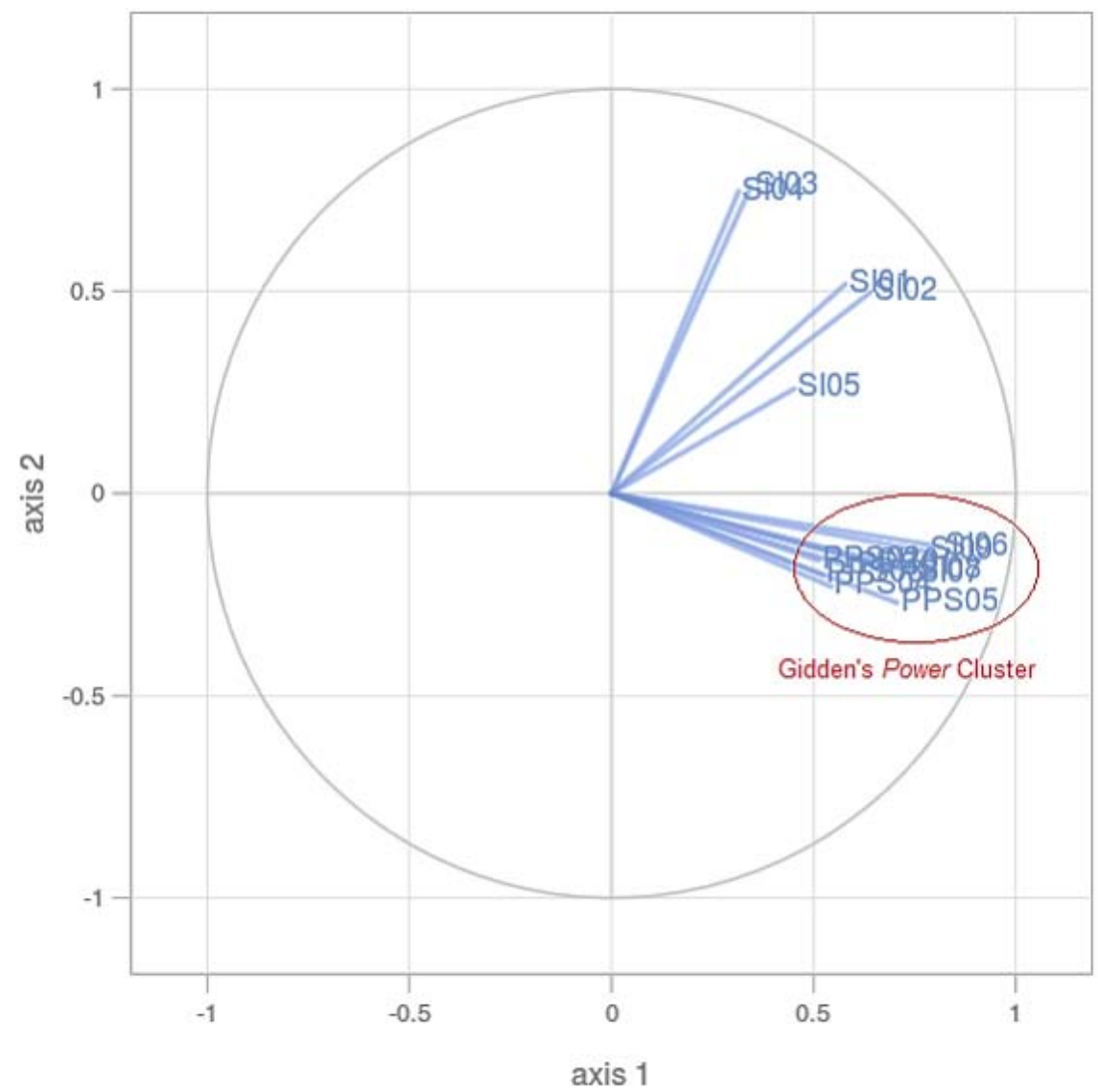

Figure 7.7: Circle of Correlations: combined social influence and perceived power security measurement items reveal a shared PCA cluster associated with Giddens' structurational power interaction. 
cluster of potential power measurement items that share a similar PCA alignment. Nonetheless, it was already known from Figure 7.6 that the PPS items themselves did not cluster well as a group. The next step was to focus solely on the larger set of power items in an effort to select the tightest cluster for further analysis.

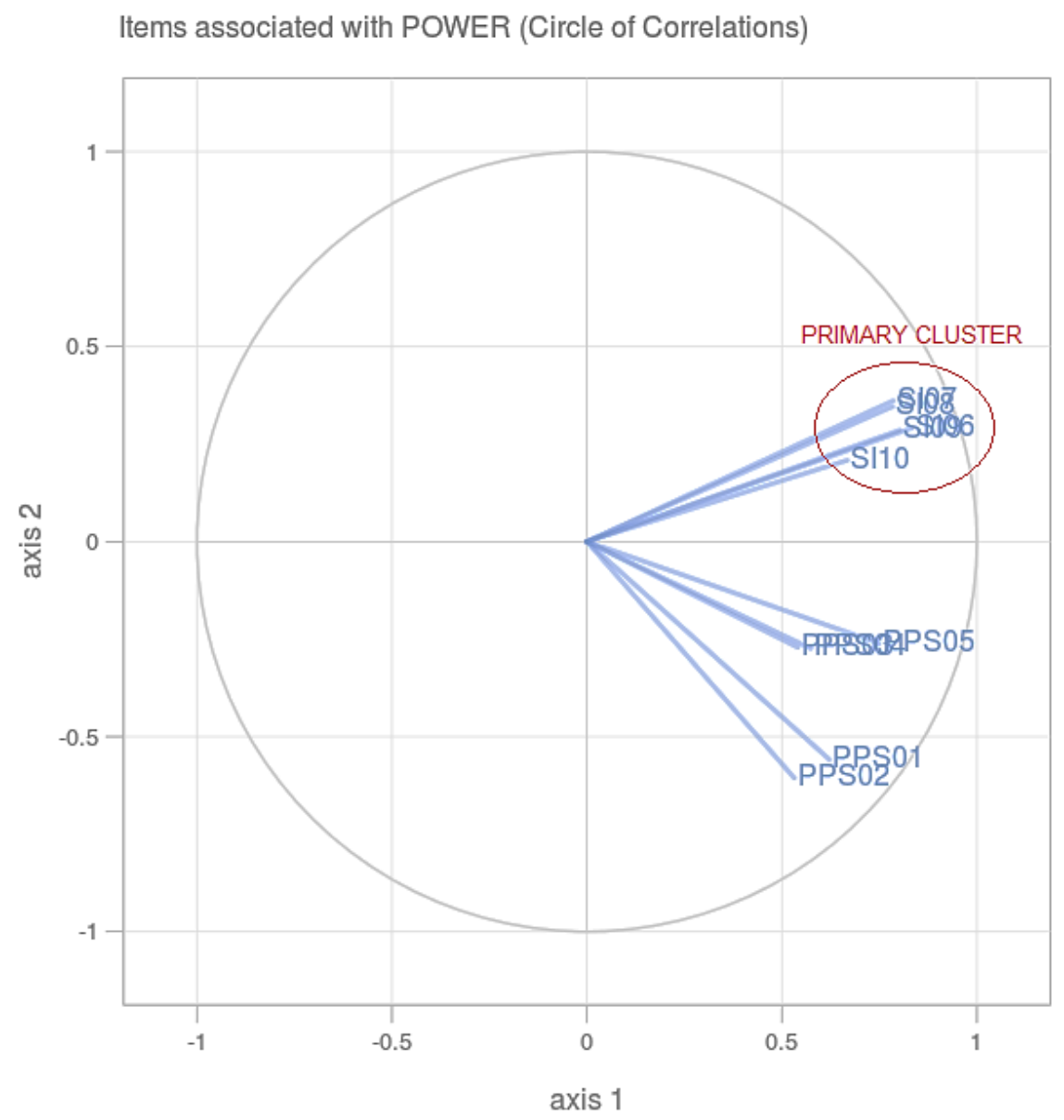

Figure 7.8: Circle of Correlations: combined power-related measurement items sourced from SI and PPS reveal that the SI-based power items form the primary cluster.

Figure 7.8 shows the circle of correlations that include only the set of SI and PPS power measurement items. The power-related SI items are still tightly clustered; however, the PPS items are once again spread outside of the 
ideal clustering, forming two additional groups. These groups were considered separately to determine if the items provided additional support to the power construct.

PPS01: Use of the system decreases my power over others; and PPS02: By recording my knowledge in the system, I will be less valuable to the organization as a source of knowledge were clustered and semantically dealt with an internal sense of personal value associated with use. Unlike the SI measurement items that dealt with a direct cause and effect, these PPS measurement items measure power indirectly by requiring an intermediary concept: the power value of knowledge. To reduce error associated with potential differences in interpretation of the power value of knowledge (a different construct from the perceived value of records), PPS01 and PPS02 were removed from the power measurement item pool.

PPS03, PPS04, and PPS05 were clustered, but were not logically related. They included measurement items that dealt with allocative control over one's records; risk of judgment and exposure; and increasing influence over others. Their principal component clustering may be due to other factors outside of the Giddens' social power interaction. PPS03, PPS04 and PPS05 were also removed from the power measurement item pool.

The PPS construct (having been stripped of all of its original measurement items) was provided with a new set of measurement items taken from the social influence measurement item pool (coinciding with Figure 7.5 Group 3). To signify this change in measurement item pool, the construct label was updated to Power (POW). This replacement construct retained the original PPS definition and theoretical foundation, but replaced the measurement items with a new set that exhibited better PCA alignment.

\section{Perceived Value of Records (PVR) Factor Analysis}

The perceived value of records was the final construct to be analyzed using PCA. Once again, the measurement item data from the survey were analyzed, resulting in a new PVR circle of correlations. See Figure 7.9.

Figure 7.9 shows two distinct clusters that emerged from the PVR measurement item pool. As with the social influence clusters, the literature was consulted in an effort to provide a theoretical foundation to explain the differences between the clusters. During the initial development of the measurement items, emergent themes were considered through the lens of Upward's Records Continuum Model (see the Records Continuum Model in Figure 2.3 on p. 24), particularly the continuum of evidentiality. With the 


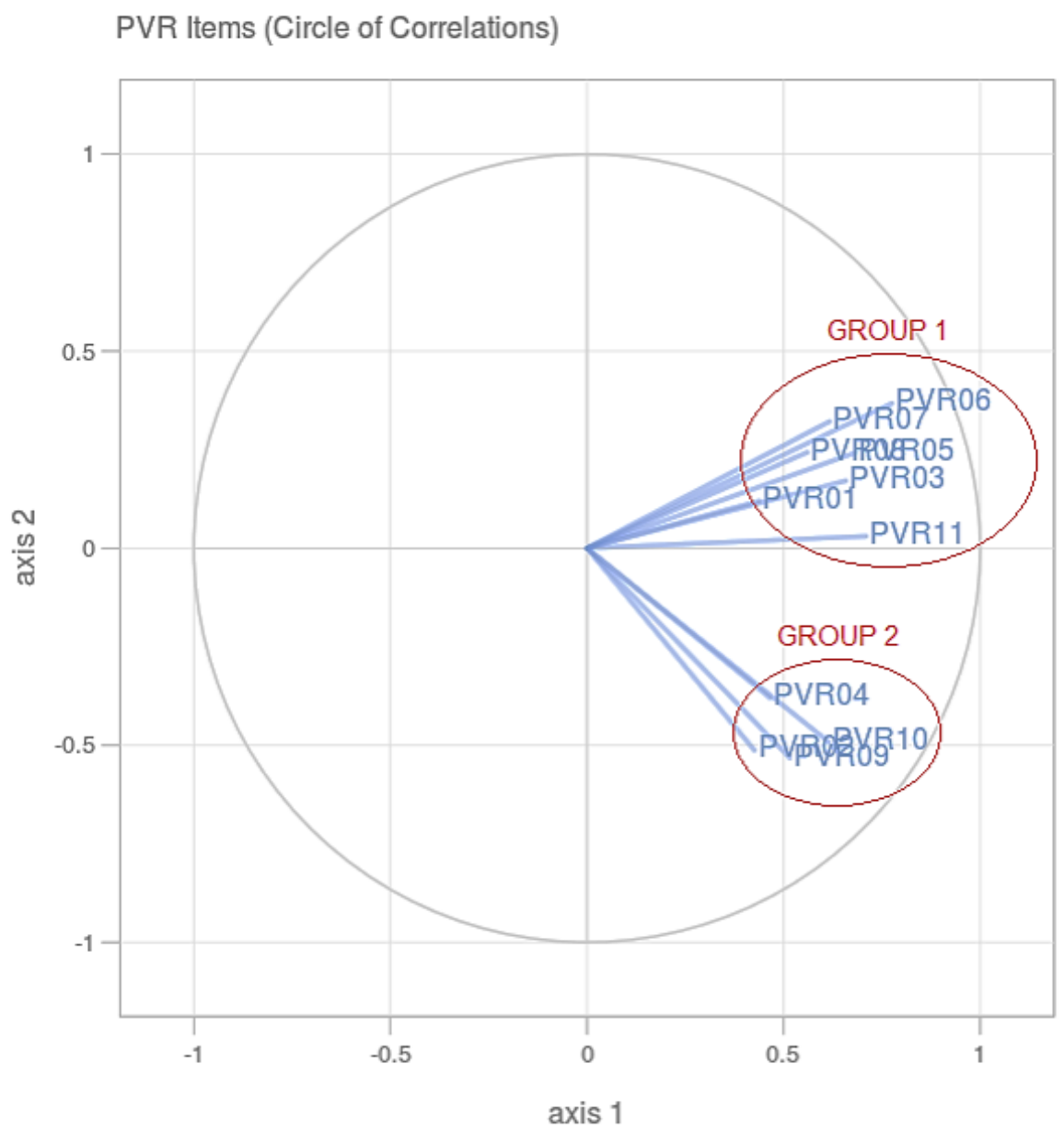

Figure 7.9: Circle of Correlations: Perceived Value of Records measurement items, resulting in two distinct clusters. Note: PVR02 was reverse-coded in order to provide a corrected directional alignment required for analysis and interpretation. 
Perceived Value of Records (PVR): PCA-based Item Selection

\begin{tabular}{|c|c|c|}
\hline \multicolumn{2}{|c|}{ PCA Group 1} & Comments \\
\hline PVR01 & $\begin{array}{l}\text { Records management is everyone's } \\
\text { responsibility. }\end{array}$ & \multirow{7}{*}{$\begin{array}{l}\text { Organizational Memory } \\
\text { (Content): } \\
\text { PCA Group } 1 \text { reflects the } \\
\text { Records Continuum's } \\
\text { Organizational Memory } \\
\text { aspect of the continuum of } \\
\text { evidentiality. Retain items in } \\
\text { pool. }\end{array}$} \\
\hline PVR03 & $\begin{array}{l}\text { Records management is an essential } \\
\text { part of my work. }\end{array}$ & \\
\hline PVR05 & $\begin{array}{l}\text { I rely on records to remind me of } \\
\text { details of my earlier work. }\end{array}$ & \\
\hline PVR06 & $\begin{array}{l}\text { I regularly refer to records to obtain } \\
\text { information needed in my job. }\end{array}$ & \\
\hline PVR07 & $\begin{array}{l}\text { Particularly during times of high staff } \\
\text { turnover, records are necessary to } \\
\text { provide continuity to my organiza- } \\
\text { tion's business processes. }\end{array}$ & \\
\hline PVR08 & $\begin{array}{l}\text { I save my records into the system } \\
\text { with the thought that someone in } \\
\text { the future will read them. }\end{array}$ & \\
\hline PVR11 & $\begin{array}{l}\text { Well-managed records improve ac- } \\
\text { countability. }\end{array}$ & \\
\hline
\end{tabular}

\begin{tabular}{|c|c|c|}
\hline \multicolumn{2}{|c|}{ PCA Group 2} & Comments \\
\hline PVR02* & $\begin{array}{l}\text { Records management requirements } \\
\text { are a barrier to working efficiently. }\end{array}$ & \multirow{4}{*}{$\begin{array}{l}\text { Trace/Evidence } \\
\text { (Context): } \\
\text { PCA Group } 2 \text { does not reflect } \\
\text { the trace/evidence aspects of } \\
\text { the Records Continuum } \\
\text { of evidentiality well. } \\
\text { Drop from item pool. }\end{array}$} \\
\hline PVR04 & $\begin{array}{l}\text { I make sure all my work-related } \\
\text { emails are filed appropriately. }\end{array}$ & \\
\hline PVR09 & $\begin{array}{l}\text { I have used records to provide reliable } \\
\text { evidence of my personal accomplish- } \\
\text { ments. }\end{array}$ & \\
\hline PVR10 & $\begin{array}{l}\text { My organization relies on records as } \\
\text { a way to achieve one or more of its } \\
\text { goals. }\end{array}$ & \\
\hline
\end{tabular}

*Reverse-coded: '. . facilitates working efficiently'

Table 7.4: Perceived Value of Records (PVR): Semantic analysis of the two PCA clusters of PVR measurement items as they reflect Upward's Records Continuum Model's continuum of evidentiality. 
literature in mind, the emergent interview themes were originally grouped into three main dimensions: ownership of accountability (trace), need or value of organizational memory, and need for reliable evidence.

In considering the two emergent clusters from the principal component analysis, the measurement items were again considered through the lens of the Records Continuum Model. The full text of the measurement items making up the two groups are documented in Table 7.4. Group 1 reflected the need and importance of records as a method to capture their information for use in the future. The importance of the content of the records is in alignment with the dimension of organizational memory within the continuum of evidentiality.

Group 2, however, did not reflect the semantic aspects of the dimensions of trace and/or evidence as strongly, nor did it reflect the value of the content of the records. Instead, Group 2 appeared to consist of a mixed bag of items ranging from the willingness to save individual emails to the perception of records management requirements as a barrier to working efficiently. Only PVR09 clearly connected a measurement item to the dimension of evidence, and unfortunately a single measurement item was insufficient to introduce a new dimensional construct into the model. This is an area to potentially investigate in future research.

Consequently, PVR02, PVR04, PVR09 and PVR10 were removed from the PVR measurement item pool, and the perceived value of records construct was instead focused on the remaining Records Continuum dimension of organizational memory as the sole dimension of interest for this research.

In this section, PCA was applied to the measurement item pools associated with each of the main constructs. The results were analyzed using the circle of correlations as a method of identifying measurement items with principal component factors that were aligned and clustered. Based on this analysis, those measurement items both closely clustered with each other and that were also semantically related to the targeted construct were retained. Those outside of the primary cluster were reconsidered in light of these two selection criteria and, in some cases, were dropped from the measurement model.

In the next section, Partial Least Squares Path Modeling (PLS-PM) is introduced, and those measurement items remaining in the construct pools were combined to form the measurement model, which supported the evaluation of the research question. 


\subsection{Partial Least Squares Path Modeling}

Partial Least Squares Path Modeling (PLS-PM) "is a statistical approach for modeling complex multivariable relationships among observed and latent variables" (Vinzi et al., 2010, p. 2). It falls within the general category of structural equation modeling (SEM) techniques.

When applied correctly, "SEM-based procedures have substantial advantages over first-generation techniques such as principal component analysis, discriminant analysis, or multiple regression because of the greater flexibility that a researcher has for the interplay between theory and data" (Chin, 1998a, p. vii). Haenlein and Kaplan (2004, p. 284) go so far as to state that the above mentioned first-generation techniques are "only applicable when there is neither a systematic nor a random error component - a rare situation in reality."

SEM-based procedures allow researchers to construct latent variables and statistically test a priori theoretical models against empirical data; in other words, it is an excellent tool for confirmatory analysis (Chin, 1998a). SEM provides particular value due to its "increased rigor" surrounding construct validity, where it provide substantial improvements to testing many subdimensions of construct validity, including "unidimensionality, convergent validity, discriminant validity, and predictive validity" (Garver \& Mentzer, 1999, p. 33). In addition, these measures greatly assisted in providing support and validation for the perceived value of records.

\subsubsection{Selection of PLS-PM}

SEM generally falls into two camps: Covariance Structure Analysis (CSA) and Partial Least Squares Path Modeling (PLS-PM). PLS-PM was selected as the more appropriate analysis method for this research primarily due to the advantages and characteristics listed below. ${ }^{2}$

- Philosophy of soft modeling: Soft modeling refers to "the ability of PLS to exhibit greater flexibility in handling various modeling problems in

\footnotetext{
${ }^{2}$ On his website dedicated to PLS modeling, Sanchez (2013a) compares and contrasts CSA to PLS-PM across 23 different dimensions, ranging from the "fitting algorithm" through to the method for estimating model correctness. PLS-PM clearly fits the context of this research better than CSA. Furthermore, many of the limitations of PLS-PM, such as its inability to handle recursive models, were of little concern given the straightforward reflective structure of the research model.
} 
situations where it is difficult or impossible to meet the hard assumptions of more traditional multivariate statistics" (Vinzi et al., 2010, p. 2). This is particularly true in relation to statistical "distributional assumptions" that are quite stringent for covariance-based approaches.

- Sample size: PLS-PM can provide significant results on substantially smaller sample sizes (Vinzi et al., 2010). Sanchez (2013a) suggests that PLS-PM will perform well with as few as 30-100 cases, whereas the sample sizes required for CSA are often larger and can require from 200-800 cases to return similar results.

- Predictive capability: PLS-PM seeks "optimal linear predictive relationships rather than for causal mechanisms, thus privileging a predictionrelevance oriented discovery process to the statistical testing of causal hypotheses" (Vinzi et al., 2010, p. 2). In other words, it is more datadriven and provides more emphasis on its measurement items than on the structural model, thereby improving its predictive capability.

In addition, Chin (1998b, p. 299) suggested a straightforward set of criteria for choosing between PLS and CSA modeling approaches:

Three essential distinctions to be brought forth between the PLS and covariance-based approaches are (a) whether the underlying constructs are modeled as indeterminate or determinate, (b) the extent to which one is confident in one's theoretical model and auxiliary theory linking measures to constructs, and (c) whether one is parameter oriented or prediction oriented. If, in each instance, one's answer is the latter, then the PLS approach is likely more suitable.

In considering Chin's SEM-method selection criteria, PLS-PM was found to be more suitable. Mirroring his above selection structure: (a) the constructs, which were reflected by the selection of measurement items, were being modeled in an effort to obtain determinate values within the latent variables that could then be used for predictive purposes; (b) the level of confidence in the theoretical model was moderated by the inclusion of a new, and untested, construct (perceived value of records); and (c) the model was predictionoriented, in that one of the objectives of the research was to develop and validate a model in order "to determine the relative importance of the factors that influence a user's intention to use an electronic recordkeeping system" 
with the intention being to use those factors to predict use. PLS-PM was thus confirmed as the preferred analysis technique for this research.

\subsubsection{PLS-PM Described}

A PLS-PM model can be considered as two separate models. The first model is the outer (measurement) model and it consists of the pools of measurement items. The measurement items are also called manifest variables since they are made manifest through direct measurement using the survey instrument. The measurement model attempts to form the latent variables (or constructs) by reflecting aspects of the measurement items using a technique based on principal component analysis. The latent variables (constructs) cannot be measured directly: they are an abstract representation. The estimate of this latent variable is simply a linear combination of their manifest variables. More precisely, the latent variables are estimated as a weighted sum of their indicators (Sanchez, 2013b, p. 39).

The second model (also called the structural or inner model) consists of the relationships between the estimated latent variables (the model's constructs). The relationships to be tested are based on the hypothesized paths between the constructs. These expected paths (research propositions) were sourced from the literature and are the basis for the research model. The research propositions were covered in detail in Chapter 3.

Sanchez (2013b) describes the general logic behind the PLS-PM algorithm. The algorithm has three major stages. Stage 1 estimates the weights necessary to compute the latent variables scores. Stage 2 estimates the path coefficients (the measure of the inner model), and stage 3 computes the loadings (the measure of the outer model).

In order to estimate the weights for the latent variables (stage 1), PLS-PM performs an iterative estimation approach. Successive iterations refine the weight estimates between the internal and external approximations of the latent variables until they reach convergence. Once the stage 1 weights have converged, the stage 2 and stage 3 calculations complete the PLS-PM model.

There are many in-depth sources that go into great detail concerning the mathematical basis and implementation of the PLS-PM algorithm (Sanchez, 2013b; Tenenhaus, Vinzi, Chatelin, \& Lauro, 2005; Chin, 1998b), and most notably Vinzi et al. (2010)'s Handbook of Partial Least Squares.

With the PLS-PM algorithm having been validated and incorporated into several different reliable and popular statistical software tools, the focus of the remainder of the analysis was on the interpretation and application of 
the outcome of the algorithm. The software packages selected to conduct the analysis and produce the relevant tables were plspm (Sanchez, Trinchera, \& Russolillo, 2013) and plsdepot (Sanchez, 2012b) as implemented in the R-Statistics platform (R Core Team, 2013).

\subsubsection{PLS-PM Outer (Measurement) Model Analysis}

In this section, the creation and testing of the outer model is described. This includes the steps taken to verify that each measurement item suitably reflected its parent construct.

The outer model was first defined in the software by allocating each of the measurement items into their construct pools (called blocks in plspm). For example, the social influence block consisted of: $\mathrm{SI}=\{\mathrm{SI01}, \mathrm{SI} 02, \mathrm{SI} 05\}$. The algorithm then approximated the latent variables and calculated the loadings for the outer model.

In terms of model fit, the loading of each measurement item is the most important measure of the contribution of that measurement item to the model. The calculated outer model loadings are listed in Table 7.5, and visually plotted in Figure 7.10 for each of the latent constructs. Note that Table 7.5 includes both the weight and loading calculations for each of the measurement items, but it also introduces two additional measures of the quality of that fitting function; namely, communality and redundancy. These are defined below:

Communality is calculated in order to "check that [the] indicators in a block are well explained by its latent variable" (Sanchez, 2013b, p. 62). It is the square of the loading $\left(\right.$ loading $\left.^{2}\right)$, and it measures the percentage of variance explained by the latent variable. It is interpreted as the reliability of the measurement item. For example, the latent construct social influence explains $\approx 67 \%$ of the observed variability of the SI01 measurement item. ${ }^{3}$

Redundancy is a measure of the "amount of variance in an endogenous construct explained by its independent latent variables" (Sanchez, 2013b,

\footnotetext{
${ }^{3}$ In the case of single measurement items, communality "is not informative about validity" (Henseler \& Sarstedt, 2013, p. 570). However, a communality index (such as mean communality - also known as Average Variance Extracted (AVE)), which assesses the whole measurement model's combined communality can be used to assess the quality of the entire outer model and is a key component of the Goodness-of-Fit indicator (Vinzi et al., 2010, p. 57).
} 


\section{OUTER MODEL: MEASURES OF FIT}

\begin{tabular}{|c|c|c|c|c|}
\hline & Weight & Loading & Communality & Redundancy \\
\hline \multicolumn{5}{|c|}{ Social Influence (Communication) } \\
\hline SI01 & 0.364 & 0.818 & 0.670 & 0.000 \\
\hline $\mathrm{SI0} 2$ & 0.393 & 0.845 & 0.713 & 0.000 \\
\hline SI05 & 0.492 & 0.752 & 0.565 & 0.000 \\
\hline \multicolumn{5}{|c|}{ Social Influence (Power) } \\
\hline SI06 & 0.247 & 0.857 & 0.734 & 0.000 \\
\hline $\mathrm{SI07}$ & 0.406 & 0.873 & 0.763 & 0.000 \\
\hline SI08 & 0.030 & 0.755 & 0.570 & 0.000 \\
\hline SI09 & 0.098 & 0.781 & 0.610 & 0.000 \\
\hline SI10 & 0.419 & 0.800 & 0.639 & 0.000 \\
\hline \multicolumn{5}{|c|}{ Perceived Value of Records } \\
\hline PVR03 & 0.268 & 0.698 & 0.488 & 0.000 \\
\hline PVR06 & 0.243 & 0.772 & 0.595 & 0.000 \\
\hline PVR07 & 0.183 & 0.671 & 0.450 & 0.000 \\
\hline PVR08 & 0.418 & 0.765 & 0.586 & 0.000 \\
\hline PVR11 & 0.265 & 0.692 & 0.479 & 0.000 \\
\hline \multicolumn{5}{|c|}{ Effort Expectancy } \\
\hline EE01 & 0.190 & 0.846 & 0.715 & 0.009 \\
\hline EE02 & 0.191 & 0.892 & 0.796 & 0.010 \\
\hline EE03 & 0.235 & 0.890 & 0.792 & 0.010 \\
\hline EE04 & 0.175 & 0.855 & 0.732 & 0.009 \\
\hline EE06 & 0.186 & 0.805 & 0.648 & 0.008 \\
\hline EE08 & 0.192 & 0.834 & 0.696 & 0.009 \\
\hline \multicolumn{5}{|c|}{ Performance Expectancy } \\
\hline PE01 & 0.228 & 0.815 & 0.665 & 0.390 \\
\hline PE02 & 0.238 & 0.890 & 0.792 & 0.464 \\
\hline PE03 & 0.233 & 0.904 & 0.817 & 0.479 \\
\hline PE06 & 0.148 & 0.662 & 0.439 & 0.257 \\
\hline PE07 & 0.177 & 0.780 & 0.609 & 0.357 \\
\hline PE10 & 0.189 & 0.822 & 0.675 & 0.396 \\
\hline \multicolumn{5}{|c|}{ Intention to Use } \\
\hline ITU01 & 0.220 & 0.832 & 0.693 & 0.357 \\
\hline ITU02 & 0.217 & 0.894 & 0.799 & 0.412 \\
\hline ITU03 & 0.208 & 0.911 & 0.830 & 0.428 \\
\hline ITU04 & 0.233 & 0.855 & 0.730 & 0.377 \\
\hline ITU05 & 0.253 & 0.928 & 0.860 & 0.444 \\
\hline
\end{tabular}

Table 7.5: Outer Model Measures of Fit. 

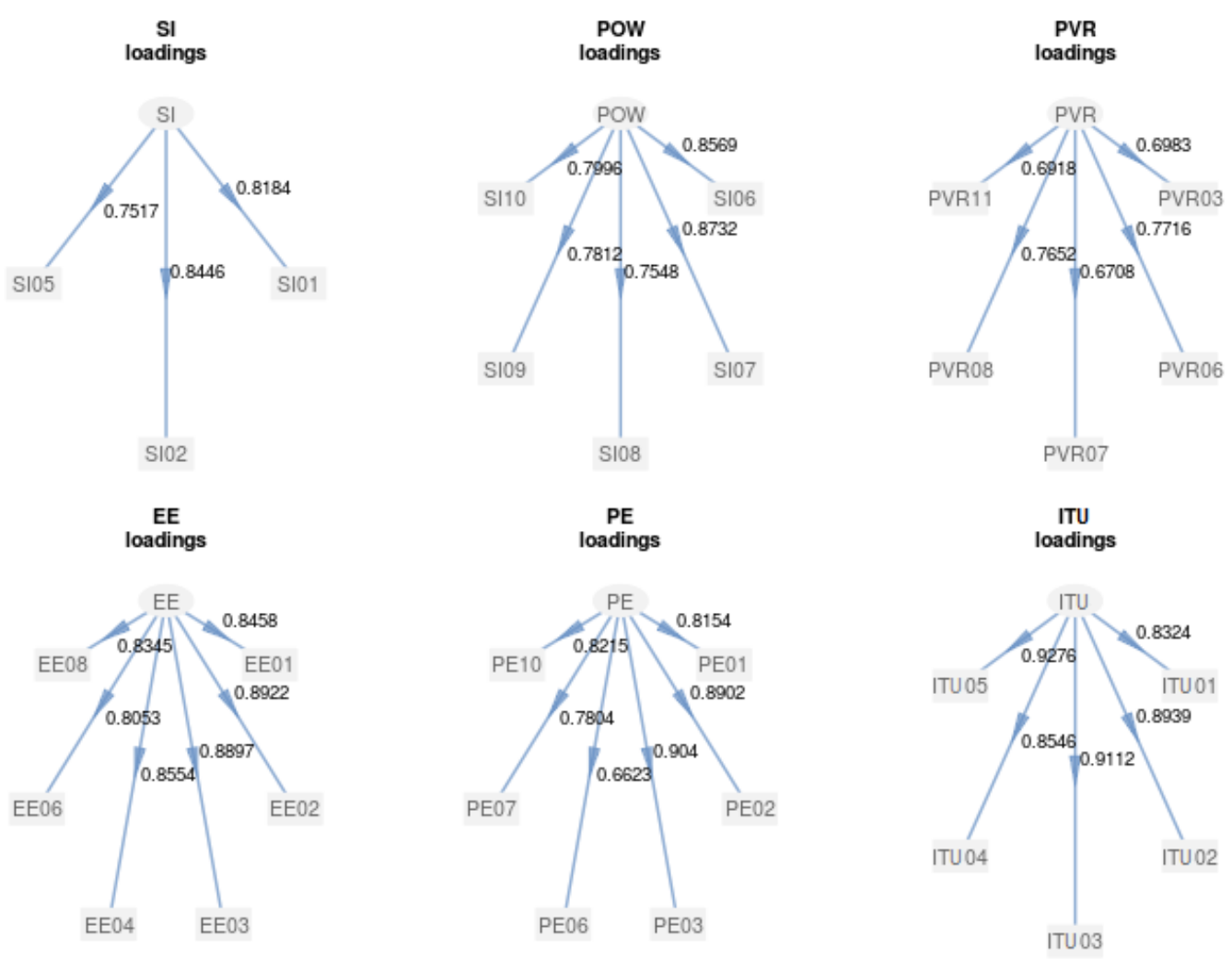

Figure 7.10: Loadings of the final selections of Outer Model Measurement Items by Construct. 
p. 65). It is only calculated for endogenous constructs, and is interpreted as: "a higher value means a higher ability to predict." 4

The redundancy definition above referred to an endogenous construct. An endogenous construct is one that acts as a dependent variable in the model path relationships, whereas an exogenous construct serves the role as an independent variable. Sometimes, a construct can serve in both roles.

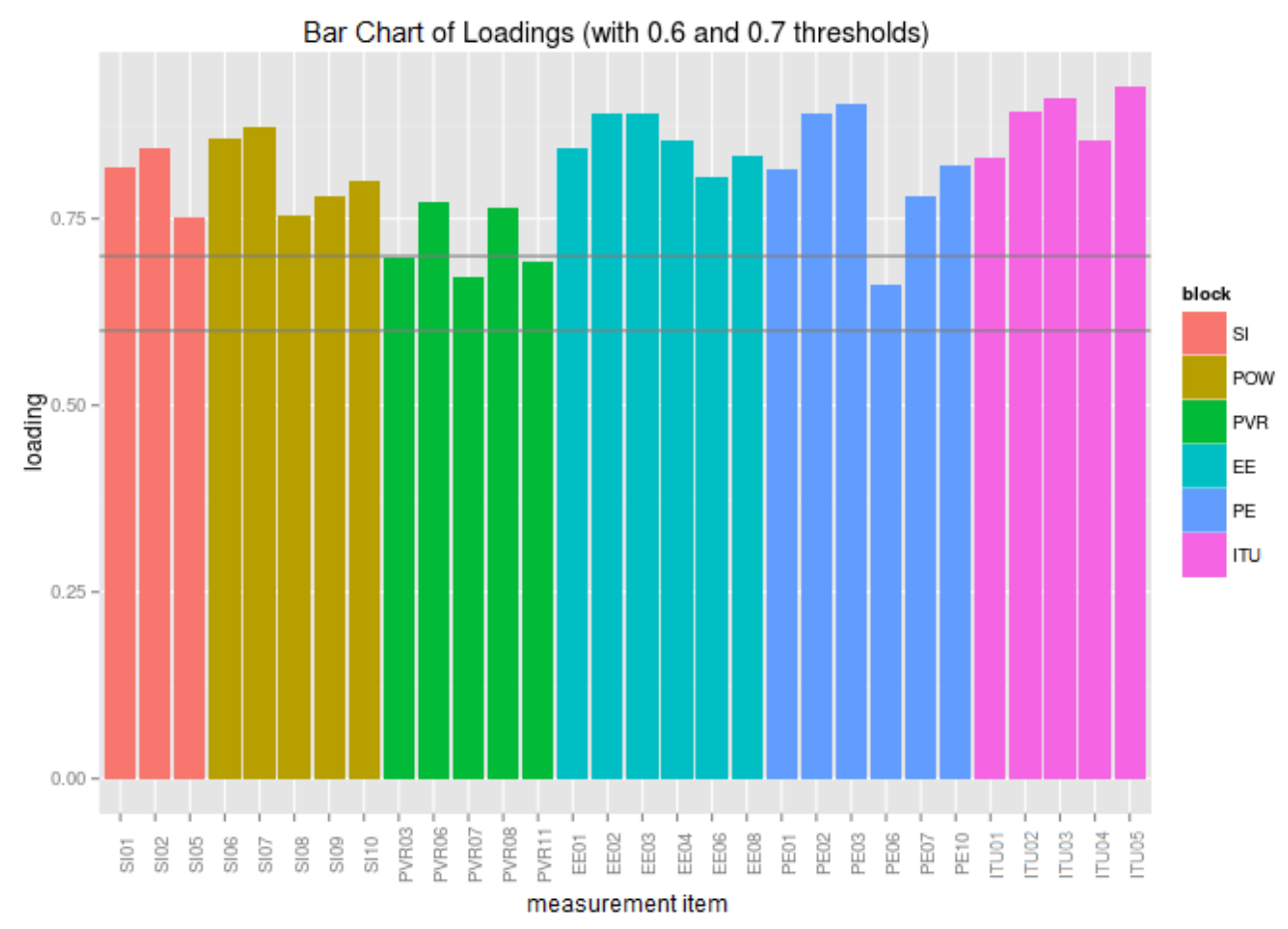

Figure 7.11: Measurement Item Loadings (with thresholds of 0.6 and 0.7 indicated). The four measurement items that were $\leq 0.7$ were retained in the model contingent on exceeding the statistical tests of unidimensionality.

Figure 7.11 better illustrates the magnitude of the loadings by displaying them in the form of a bar chart indicating thresholds at the 0.6 and 0.7 levels. Loadings that are $<0.7$ may be acceptable (can contribute to the model), as long each construct block is able to exceed thresholds for statistical

\footnotetext{
${ }^{4}$ Similarly to the communality index, a redundancy index can be calculated as a measure of the prediction performance of the measurement model (Vinzi et al., 2010, p. 58).
} 
tests of unidimensionality across its pool of measurement items. ${ }^{5}$ Figure 7.11 highlights four measurement items that fell within the range: $0.6 \leq X \leq 0.7$. After reviewing the measurement items, it was decided to retain all of them in the model, as each of them contained semantically important aspects that were expected to add to the interpretability of the model.

Having verified that the loadings were generally within tolerances, the PLSPM statistical tests for unidimensionality were then calculated. See Table 7.6. These tests considered different aspects of how well the calculated model explained its measurement item pool. As recommended by Chin (2010b), the model was also checked by verifying the inter-construct correlations and reliability measures (see Table 7.7 ) and verifying that the desired loadings were larger than any cross-loadings (see Table 7.8).

\begin{tabular}{lcccccc}
\multicolumn{2}{l}{ Blocks Unidimensionality } \\
\hline & Mode & MVs & C.alpha & DG.rho & eig.1st & eig.2nd \\
\hline SI & A & 3 & 0.733 & 0.852 & 1.99 & 0.764 \\
POW & A & 5 & 0.891 & 0.921 & 3.50 & 0.634 \\
PVR & A & 5 & 0.776 & 0.848 & 2.65 & 0.785 \\
EE & A & 6 & 0.926 & 0.942 & 4.38 & 0.472 \\
PE & A & 6 & 0.898 & 0.923 & 4.00 & 0.705 \\
ITU & A & 5 & 0.930 & 0.947 & 3.91 & 0.395 \\
\hline
\end{tabular}

Table 7.6: Unidimensionality Indicators: measures of the validity of the outer model indicators. All measures achieved the required thresholds.

Each of the column descriptors and statistical tests referred to in Table 7.6 are further defined and described below (including the relevant interpretation and thresholds as required).

Mode: this variable indicates the type of measurement block. In this case, all measurement blocks are Mode A or reflective measurement blocks (as opposed to formative measurement blocks - Mode B). In the case of Mode A, the latent variable has been theoretically predefined and the measurement items are evaluated on their ability to reflect the defined latent variable (Hanafi, 2007; Haenlein \& Kaplan, 2004; Chin, 1998a).

\footnotetext{
${ }^{5}$ It is desirable for the loading of each measurement item to exceed 0.7 , because its communality (which is the square of the loading) would then exceed 0.5 or $50 \%$, thereby explaining at least half of the observed variance.
} 


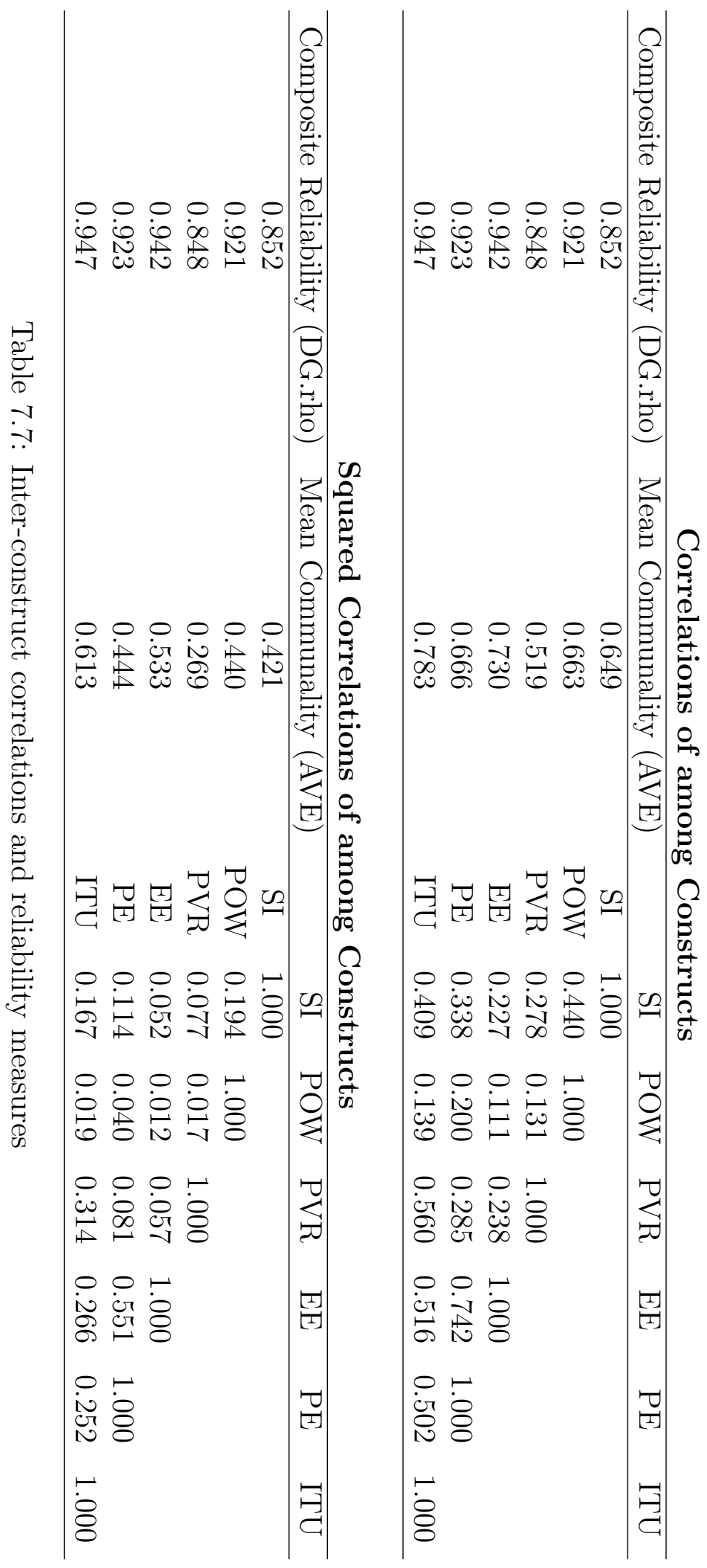


Loadings and Cross-loadings

\begin{tabular}{|c|c|c|c|c|c|c|}
\hline & SI & POW & PVR & $\mathrm{EE}$ & $\mathrm{PE}$ & ITU \\
\hline \multicolumn{7}{|c|}{ Social Influence (SI) } \\
\hline SI01 & 0.818 & 0.350 & 0.181 & 0.187 & 0.258 & 0.256 \\
\hline SI02 & 0.845 & 0.419 & 0.266 & 0.157 & 0.267 & 0.288 \\
\hline SI05 & 0.752 & 0.299 & 0.219 & 0.198 & 0.282 & 0.413 \\
\hline \multicolumn{7}{|c|}{ Perceived Power Security (POW) } \\
\hline SI06 & 0.417 & 0.857 & 0.115 & 0.054 & 0.164 & 0.096 \\
\hline SI07 & 0.358 & 0.873 & 0.085 & 0.132 & 0.206 & 0.115 \\
\hline SI08 & 0.368 & 0.755 & 0.051 & 0.019 & -0.097 & 0.001 \\
\hline SI09 & 0.385 & 0.781 & 0.062 & 0.040 & 0.083 & 0.019 \\
\hline SI10 & 0.341 & 0.800 & 0.152 & 0.094 & 0.155 & 0.160 \\
\hline \multicolumn{7}{|c|}{ Perceived Value of Records (PVR) } \\
\hline PVR03 & 0.184 & 0.075 & 0.698 & 0.187 & 0.176 & 378 \\
\hline PVR06 & 0.197 & 0.022 & 0.772 & 0.123 & 0.163 & 0.339 \\
\hline PVR07 & 0.063 & 0.054 & 0.671 & 0.062 & 0.076 & 0.302 \\
\hline PVR08 & 0.320 & 0.192 & 0.765 & 0.262 & 0.334 & 0.530 \\
\hline PVR11 & 0.136 & 0.059 & 0.692 & 0.139 & 0.171 & 0.376 \\
\hline \multicolumn{7}{|c|}{ Effort Expectancy (EE) } \\
\hline EE01 & 0.149 & 0.096 & 0.093 & 0.846 & 0.640 & 0.393 \\
\hline EE02 & 0.224 & 0.062 & 0.228 & 0.892 & 0.611 & 0.463 \\
\hline EE03 & 0.241 & 0.139 & 0.236 & 0.890 & 0.750 & 0.511 \\
\hline EE04 & 0.188 & 0.098 & 0.190 & 0.855 & 0.574 & 0.368 \\
\hline EE06 & 0.162 & 0.029 & 0.242 & 0.805 & 0.602 & 0.479 \\
\hline EE08 & 0.190 & 0.133 & 0.221 & 0.835 & 0.599 & 0.413 \\
\hline \multicolumn{7}{|c|}{ Performance Expectancy (PE) } \\
\hline PE01 & 0.215 & 0.094 & 0.306 & 0.613 & 0.815 & 0.557 \\
\hline $\mathrm{PE} 02$ & 0.333 & 0.217 & 0.291 & 0.659 & 0.890 & 0.478 \\
\hline PE03 & 0.338 & 0.251 & 0.258 & 0.682 & 0.904 & 0.449 \\
\hline PE06 & 0.212 & 0.153 & 0.167 & 0.471 & 0.662 & 0.247 \\
\hline PE07 & 0.266 & 0.131 & 0.185 & 0.550 & 0.780 & 0.313 \\
\hline PE10 & 0.277 & 0.121 & 0.153 & 0.631 & 0.822 & 0.340 \\
\hline \multicolumn{7}{|c|}{ Intention to Use (ITU) } \\
\hline ITU01 & 0.325 & 0.091 & 0.479 & 0.453 & 0.478 & 0.832 \\
\hline ITU02 & 0.378 & 0.111 & 0.448 & 0.446 & 0.414 & 0.894 \\
\hline ITU03 & 0.351 & 0.100 & 0.476 & 0.388 & 0.404 & 0.911 \\
\hline ITU04 & 0.340 & 0.142 & 0.467 & 0.515 & 0.461 & 0.855 \\
\hline ITU05 & 0.410 & 0.164 & 0.591 & 0.471 & 0.458 & 0.928 \\
\hline
\end{tabular}

Table 7.8: Loadings and cross-loadings for the outer (measurement) model 
MV (Manifest Variables): the number of manifest variables ${ }^{6}$ or measurement items that are included in the block associated with each latent variable or construct.

C.alpha (Cronbach's Alpha): [goal: > 0.7] is a classic coefficient to measure internal consistency. "A block is considered homogenous [unidimensional] if this index is larger than 0.7 for confirmatory studies" (Vinzi et al., 2010, p. 50).

DG.rho (Dillon-Goldstein's rho): [goal: > 0.7] (also called composite reliability) it is another measure of block homogeneity. In the context of PLS-PM, Dillon-Goldstein's rho is considered a better indicator than Cronbach's Alpha because it bases its estimates on the PLS-PM loadings directly instead of relying on the measurement item correlation matrix of the data. "A block is considered homogenous [unidimensional] if this index is larger than 0.7" (Vinzi et al., 2010, p. 50).

eig.1st (1st Eigenvalue): [goal: $>1.0$ ] is also known as the 1st principal component. A high value (significantly greater than one) indicates that the majority of the correlation matrix of measurement items is based on the importance of the first eigenvalue, and is therefore unidimensional (Vinzi et al., 2010, p. 50).

eig.2nd (2nd Eigenvalue): [goal: $<1.0$ ] is also known as the 2nd principal component. This is a double-check of the power of the 1st Eigenvalue. A very low value (less than 1) [Kaiser's Rule], which indicates that the remainder of the correlation matrix is unimportant and is therefore unidimensional (Vinzi et al., 2010, p. 50).

With regard to the tests of unidimensionality, all measures presented in Table 7.6 were well within the established thresholds. Therefore, the outer model was considered good and was able to provide the foundation for testing the inner model.

\subsubsection{PLS-PM Inner (Structural) Model Analysis}

The inner model is also known as the structural model and corresponds with the theoretical research model. It consists of the proposed relationships

\footnotetext{
${ }^{6}$ Measurement items are sometimes called manifest variables (MV) to differentiate them from a construct or latent variable (LV). In the plspm output, MV and LV are often used as labels.
} 
between the latent variables (the model's constructs). The logic of the research model's relationships as proposed in Figure 3.2 (p. 65) was re-created in the plspm software package and linked to the appropriate manifest variables (or measurement items) to support the remaining analysis.

\section{PLS-PM Path Coefficients}

When run, the resulting weights and loadings for each measurement item statistically formed estimates for the latent variables, which were then related via their designated path relationships in the inner model using a regression model. The plspm calculations provide path coefficients that are a measure of the strength and direction of the relationships between the independent and dependent variables. A plspm plot of the resulting inner model with path coefficients is shown in Figure 7.12.

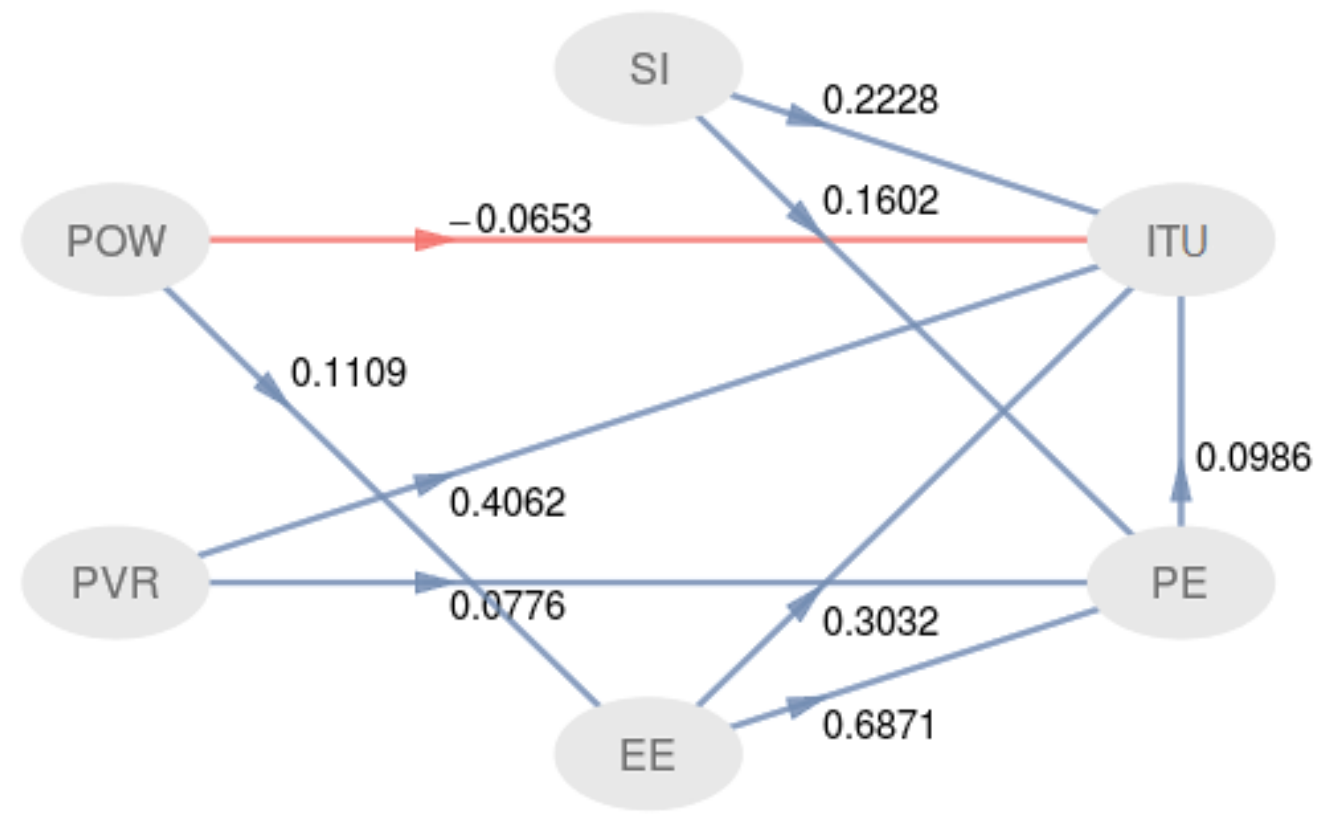

Figure 7.12: Research Model with Path Coefficients

The path coefficients in Figure 7.12 show both positive effects, designated as blue lines and positive path coefficients, and one neutral/negative effect, 


\section{Total Path Effects}

\begin{tabular}{cccc}
\hline Relationship & Direct & Indirect & Total \\
\hline $\mathrm{SI} \rightarrow \mathrm{POW}$ & 0.0000 & 0.0000 & 0.0000 \\
$\mathrm{SI} \rightarrow \mathrm{PVR}$ & 0.0000 & 0.0000 & 0.0000 \\
$\mathrm{SI} \rightarrow \mathrm{EE}$ & 0.0000 & 0.0000 & 0.0000 \\
$\mathrm{SI} \rightarrow \mathrm{PE}$ & 0.1602 & 0.0000 & 0.1602 \\
$\mathrm{SI} \rightarrow \mathrm{ITU}$ & 0.2228 & 0.0158 & 0.2386 \\
$\mathrm{POW} \rightarrow \mathrm{PVR}$ & 0.0000 & 0.0000 & 0.0000 \\
$\mathrm{POW} \rightarrow \mathrm{EE}$ & 0.1109 & 0.0000 & 0.1109 \\
$\mathrm{POW} \rightarrow \mathrm{PE}$ & 0.0000 & 0.0762 & 0.0762 \\
$\mathrm{POW} \rightarrow \mathrm{ITU}$ & -0.0653 & 0.0411 & -0.0241 \\
$\mathrm{PVR} \rightarrow \mathrm{EE}$ & 0.0000 & 0.0000 & 0.0000 \\
$\mathrm{PVR} \rightarrow \mathrm{PE}$ & 0.0776 & 0.0000 & 0.0776 \\
$\mathrm{PVR} \rightarrow \mathrm{ITU}$ & 0.4062 & 0.0077 & 0.4139 \\
$\mathrm{EE} \rightarrow \mathrm{PE}$ & 0.6871 & 0.0000 & 0.6871 \\
$\mathrm{EE} \rightarrow \mathrm{ITU}$ & 0.3032 & 0.0678 & 0.3710 \\
$\mathrm{PE} \rightarrow \mathrm{ITU}$ & 0.0986 & 0.0000 & 0.0986 \\
\hline
\end{tabular}

Table 7.9: Total Path Effects: the effects that a construct has on other constructs in the inner model. The direct effects (path coefficients) and the indirect effects (effect via an indirect path) can be summed to calculate the total effect. 
designated as a red line and in this case a negative path coefficient. ${ }^{7}$ In this case, the perceived value of records is seen to explain $40.6 \%$ of the observed variance associated with its relationship with the intention to use.

An independent analysis of the total path effects of the research model is shown in Table 7.9 (p. 174). These path effects are also shown graphically in Figure 8.1 on p. 203.

\subsubsection{PLS-PM Model Significance Tests}

Since the inner model as illustrated in Figure 7.12 can be interpreted as a regression model, standard path significance tests can be used to determine the confidence intervals (or significance levels) for each of the path coefficients. These are presented in Table 7.10.

Table 7.10 consists of four columns that provide measures of the significance of the path coefficients. The columns are:

Estimate: the PLS-PM estimate of the path coefficient. For example, the estimate of $P V R \rightarrow I T U$ shows PVR explains $40.62 \%$ of the observed variance of ITU.

Std. Error (Standard Error): the statistical standard deviation of the path coefficient estimate mean.

$t$ value: a single-tailed $t$-test showing the standardized $t$ score.

Probability $p(>|t|)$ : the probability calculated from the $t$ value at the $95 \%$ significance level. This probability is interpreted using the standard asterisk system with the key located at the bottom of the Table.

Table 7.10 also illustrates that the apparent negative path coefficient associated with $P O W \rightarrow I T U$ (as indicated by the red arrow in Figure 7.12) is also not statistically significant. In fact, both effects of power were found to be insignificant, including: $P O W \rightarrow I T U$ and $P O W \rightarrow E E$.

Somewhat of a surprise was the finding that performance expectancy's effect $(P E \rightarrow I T U)$ was not significant, as performance expectancy's impact

\footnotetext{
${ }^{7}$ Note that the path coefficients (which represent both the strength and direction of the relationship) require interpretation. For example, the statement "the more power I lose due to electronic recordkeeping within my organization, the more I will use the system" does not make sense. Rather, the low impact value of $P O W \rightarrow I T U$ should be interpreted as "The impact of power on intention to use is negligible."
} 


\section{Inner Model Significance Tests}

Path Loadings onto Effort Expectancy (EE)

\begin{tabular}{|l|c|c|c|c|}
\cline { 2 - 5 } \multicolumn{1}{c|}{} & Estimate & Std. Error & t value & $p(>|t|)$ \\
\hline Intercept & 0.0000 & 0.0719 & 0.0000 & 1.0000 \\
\hline POW $^{\text {ns }}$ & 0.1109 & 0.0719 & 1.5400 & 0.1250 \\
\hline
\end{tabular}

Path Loadings onto Performance Expectancy (PE)

\begin{tabular}{|c|c|c|c|c|}
\hline & Estimate & Std. Error & t value & $p(>|t|)$ \\
\hline Intercept & 0.0000 & 0.0468 & 0.0000 & 1.0000 \\
\hline SI** & 0.1602 & 0.0495 & 3.2400 & 0.0014 \\
\hline $\mathrm{PVR}^{\mathrm{ns}}$ & 0.0776 & 0.0496 & 1.5700 & 0.1190 \\
\hline $\mathrm{EE}^{* * *}$ & 0.6871 & 0.0489 & 14.0000 & 0.0000 \\
\hline
\end{tabular}

Path Loadings onto Intention to Use (ITU)

\begin{tabular}{|l|c|c|c|c|}
\cline { 2 - 5 } \multicolumn{1}{c|}{} & Estimate & Std. Error & t value & $p(>|t|)$ \\
\hline Intercept & 0.0000 & 0.0509 & 0.0000 & 1.0000 \\
\hline SI $^{* * *}$ & 0.2228 & 0.0602 & 3.7000 & 0.0003 \\
\hline POW $^{\text {ns }}$ & -0.0653 & 0.0568 & -1.1500 & 0.2520 \\
\hline PVR $^{* * *}$ & 0.4062 & 0.0543 & 7.4800 & 0.0000 \\
\hline EE $^{* * *}$ & 0.3032 & 0.0761 & 3.9800 & 0.0001 \\
\hline $\mathrm{PE}^{\mathrm{ns}}$ & 0.0986 & 0.0793 & 1.2400 & 0.2150 \\
\hline$n s=p>0.05$ & $* *=p \leq 0.01$ \\
$*=p \leq 0.05$ & $* *=p \leq 0.001$
\end{tabular}

Table 7.10: Inner Model Significance Tests 
on system use constructs is generally quite high. Finally, the new perceived value of records construct $(P V R \rightarrow I T U)$ resulted in a strong positive effect of 0.4062 , which was also highly significant $(p \leq 0.001)$. These path effects and their interpretations will be covered in more detail in Section 7.5.

\section{Quality Summary of the Structural Model}

The quality of a regression model is generally measured using the coefficient of determination $\left(R^{2}\right)$ - a statistical measure of how well the regression line approximates the actual data. For a visual illustration, a correlation matrix of the model's path coefficients has been created in Figure 7.13. This correlation matrix consists of scatter plots of the individual participants' path coefficients, which, when viewed together, either show a positive correlation (e.g., between EE and PE) or no correlation at all (e.g., between POW and PE). The $R^{2}$ value provides a measure of the goodness of fit between the regression line and its data points, and it is calculated for all endogenous variables of the structural model's designated paths.

However, as mentioned earlier, the quality measure for the outer (measurement) model is its communality, which is calculated as the index of communality (or average communality across the model). Unfortunately, the traditional measure of $R^{2}$ does not capture the quality of fit for that outer model. Therefore, a new measure for the goodness of fit for a PLS-PM model - one that reflects both the outer model and the inner model - has been proposed. Called the Goodness-of-Fit Index (GoF), it consists of the geometric mean of the average communality and the average $R^{2}$ and is interpreted similarly to $R^{2}$, as an improved indicator of the quality-of-fit for the model (Tenenhaus et al., 2005; Chin, 2010b; Sanchez, 2013b). Conceptually, Equation 7.1 illustrates how GoF combines the two primary measures of quality-of-fit together in one index.

$$
\text { GoF }=\sqrt{\overline{\text { Communality }} * \overline{R^{2}}}
$$

The final measure of the model's quality is its redundancy (see p. 165). The mean redundancy provides an indicator of the "predictive relevance of one's theoretical/structural model" (Chin, 2010b, p. 680). Table 7.11 provides a summary of the various measures for the research model's quality-of-fit.

The $R^{2}$ for the intention to use is 0.516 , which rates the model as moderate to high in its explanatory capability (Sanchez, 2013b). By incorporating the additional error associated with the mean communality, the GoF Index 


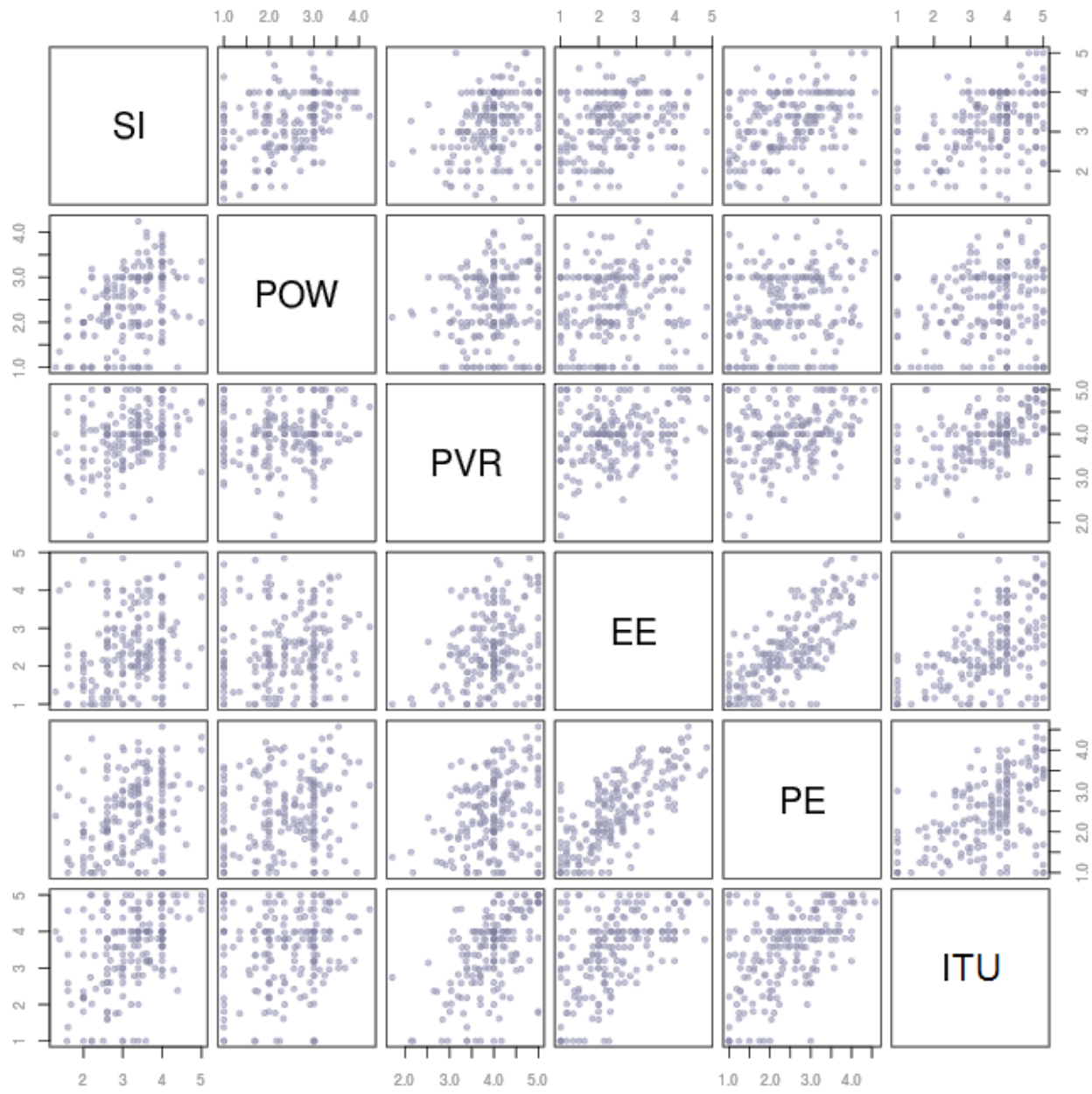

Figure 7.13: Correlation Matrix of Model Path Coefficients demonstrating positively correlated relationships and uncorrelated relationships between constructs. 
Summary: Measures of Model Fit

\begin{tabular}{|l|l|c|c|c|}
\cline { 2 - 5 } \multicolumn{1}{c|}{} & Type & $R^{2}$ & Mean Communality & Mean Redundancy \\
\hline SI & Exogenous & 0.000 & 0.649 & 0.000 \\
\hline POW & Exogenous & 0.000 & 0.663 & 0.000 \\
\hline PVR & Exogenous & 0.000 & 0.519 & 0.000 \\
\hline EE & Endogenous & 0.012 & 0.730 & 0.009 \\
\hline PE & Endogenous & 0.586 & 0.666 & 0.390 \\
\hline ITU & Endogenous & 0.516 & 0.783 & 0.404 \\
\hline \multicolumn{5}{|c|}{ Goodness-of-Fit (GoF): 0.4994} \\
\hline
\end{tabular}

Table 7.11: Quality of Model Fit: a summary of the multiple measures, including $R^{2}$ for the inner (structural) model, Mean Communality (AVE) for the outer (measurement) model, Mean Redundancy as an indicator of the predictive relevance, and the Goodness-of-Fit $(\mathrm{GoF})$ as a global criterion for the overall predictive performance of the model.

presents a more conservative value of $\approx 0.50$. This can be interpreted as the model explaining $50 \%$ of the observed variance of the intention to use.

The above parameters provide estimates of the quality-of-fit; however, they do not provide information about the variability of the parameter estimates. Also, since PLS-PM "does not rest on any distributional assumptions, significance levels for the parameter estimates (based on normal theory) are not suitable" (Sanchez, 2013b, p. 66). To provide an estimate of the variability of the parameter estimates, a resampling procedure called bootstrapping was applied.

\subsubsection{PLS-PM Bootstrapping and Model Validation}

Bootstrapping is "a non-parametric approach for estimating the precision of the PLS parameter estimates" (Sanchez, 2013b, p. 67). It essentially creates a new dataset by randomly sampling with replacement from the original dataset. By comparing the two datasets, it is possible to calculate an estimate of the standard error and thus determine the significance levels for each of the measurement items and their effect paths (Chin, 2010a).

For the bootstrapping analysis of the survey data, the sampling size was set at 200 resamples in order to mirror the approximate size of the original data set (note that the default for the plspm software package is 100 resamples). 
The results of the bootstrapping validation are shown in Appendix $\mathrm{F}$ on p. 265.

For each measurement item, the original value is compared to the mean of the resampled data, resulting in a standard error (indicating the standard deviation of the mean), and the lower and upper percentiles of the $95 \%$ bootstrap confidence interval.

Owing to the care with which the measurement items were selected in terms of their loadings, there was only one measurement item whose loading was marginally insignificant - i.e., SI08, associated with the power construct. However, all of the weights for all of the power construct measurement items were also not significant, indicating that power was not performing well within the research model.

In terms of the path effects associated with the inner model, the same paths that were found to be insignificant in the inner model significance tests as shown in Table 7.10 on p. 176, were confirmed to be insignificant in the bootstrapping validation results in Table F.3 (p. 268). Conversely, all significant paths in Table 7.10 were also validated to be significant in Table F.3.

Finally, the $R^{2}$ bootstrap validation (which calculated the mean bootstrap $R^{2}$ at a slightly higher value of 0.538 ) was found to be highly significant during resampling. See Figure 7.14 for the final measurement of the research model. 


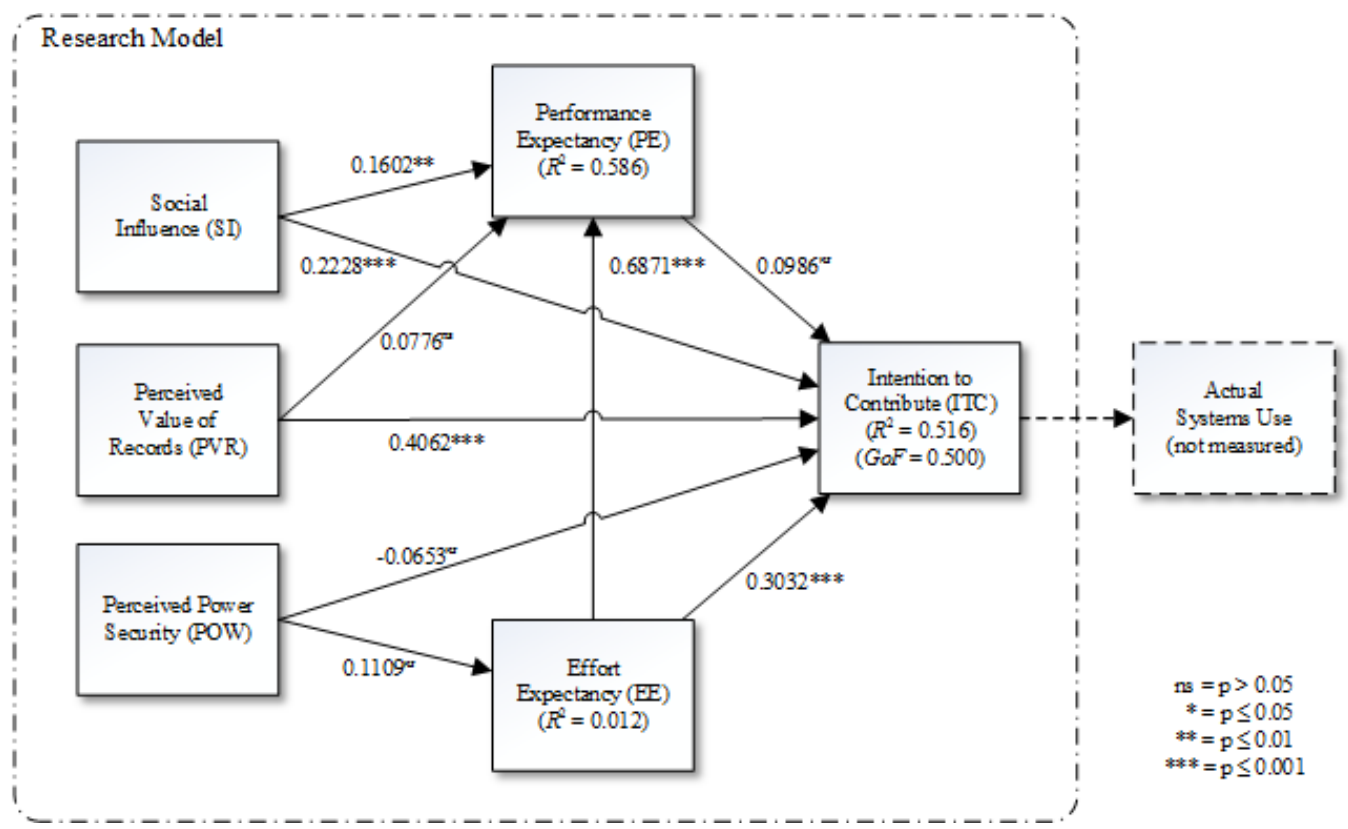

Figure 7.14: The measured research model.

\subsection{Analysis of Hypothesized Effects}

In Chapter 3, an analysis of the literature resulted in a set of nine research propositions that formed the research model. In this section, each of the research hypotheses is discussed in light of the research analysis results. In Table 7.12, each hypothesized path effect is considered using the plspm path coefficient and a measure of its statistical significance (see Table 7.10).

Research Hypothesis $\mathbf{1}$ (H1): [EE $\rightarrow$ ITU] effort expectancy (perceived ease of use) will have a positive effect on a user's intention to use an electronic recordkeeping system.

Supported - effort expectancy had both a strong and positive effect on intention to use, with a path coefficient of 0.3032 . The measure of this positive effect was highly statistically significant $(p \leq 0.001)$.

Research Hypothesis 2 (H2): $[E E \rightarrow P E]$ effort expectancy (perceived ease of use) will have a positive effect on a user's performance expectancy (perceived usefulness) of an electronic recordkeeping system. 


\begin{tabular}{ccc}
\hline Hypothesis & Impact & Result \\
\hline H1 & $E E \rightarrow I T U$ & Supported \\
H2 & $E E \rightarrow P E$ & Supported \\
H3 & $P E \rightarrow I T U$ & Not Supported \\
H4 & $S I \rightarrow P E$ & Supported \\
H5 & $S I \rightarrow I T U$ & Supported \\
H6 & $P O W \rightarrow I T U$ & Not Supported \\
H7 & $P O W \rightarrow E E$ & Not Supported \\
H8 & $P V R \rightarrow P E$ & Not Supported \\
H9 & $P V R \rightarrow I T U$ & Supported \\
\hline
\end{tabular}

Table 7.12: Research Hypotheses Results Summary

Supported - effort expectancy had a strong and positive effect on performance expectancy, with a path coefficient of 0.6871 . This effect was highly statistically significant $(p \leq 0.001)$.

Research Hypothesis 3 (H3): $[P E \rightarrow I T U]$ performance expectancy (perceived usefulness) will have a positive effect on a user's intention to use an electronic recordkeeping system.

Not supported - the analysis indicated that performance expectancy (perceived usefulness) had a very small positive effect on a user's intention to use, with a path coefficient of 0.0986. However, that small positive effect was not statistically significant $(n s)$, indicating that this hypothesized effect is not in evidence.

Research Hypothesis $4(\mathbf{H} 4):[S I \rightarrow P E]$ social influence will have a positive effect on a user's performance expectancy (perceived usefulness) of an electronic recordkeeping system.

Supported - social influence had a positive effect on a user's performance expectancy (perceived usefulness), with a path coefficient of 0.1602 . The measurement of this positive effect was statistically significant $(p \leq 0.01)$.

Research Hypothesis $\mathbf{5}$ (H5): $[S I \rightarrow I T U]$ social influence will have a positive effect on a user's intention to use an electronic recordkeeping system. 
Supported - social influence had a strong positive effect on a user's intention to use, with a path coefficient of 0.2228 . The measurement of this positive effect was highly statistically significant $(p \leq 0.001)$.

Research Hypothesis 6 (H6): $[P O W \rightarrow I T U]$ perceived power security will have a positive effect on a user's intention to use an electronic recordkeeping system.

Not supported - The analysis found that perceived power security did not have a positive effect on a user's intention to use. Instead, it found a small neutral to negative effect, with a path coefficient of 0.0653. Furthermore, the measurement of this effect was not statistically significant $(n s)$, indicating that this hypothesized effect was not in evidence.

Research Hypothesis $\mathbf{7}(\mathbf{H 7}):[P O W \rightarrow$ EE] perceived power security will have a positive effect on a user's effort expectancy (perceived ease of use) of an electronic recordkeeping system.

Not supported - the analysis found evidence that perceived power security had a positive effect on a user's effort expectancy (perceived ease of use), with a path coefficient of 0.1109 . But the variance of the measurements indicate that this positive effect was not statistically significant $(n s)$. The combination of $\mathrm{H} 6$ and $\mathrm{H} 7$ draws into question the actual effect of power as a factor in the research model.

Research Hypothesis $\mathbf{8}$ (H8): $[P V R \rightarrow P E]$ perceived value of records will have a positive effect on a user's performance expectancy (perceived usefulness) of an electronic recordkeeping system.

Not supported - the analysis found that perceived value of records exhibited a very small positive effect on a user's performance expectancy (perceived usefulness), with a path coefficient of 0.0776. However, the variance of the measurements indicate that this measured positive effect was not statistically significant $(n s)$.

Research Hypothesis 9 (H9): $[P V R \rightarrow I T U]$ perceived value of records will have a positive effect on a user's intention to use an electronic recordkeeping system.

Supported - perceived value of records had a very strong positive effect on a user's intention to use, with a large and positive path coefficient of 0.4062 . The effect was highly statistically significant $(p \leq 0.001)$. 
As mentioned at the beginning of this section, the hypothesized effects between the constructs contributed to forming the paths of the research model. The results of the survey were then used to confirm those proposed effects. In general, the proposed effects were confirmed, with a few noted exceptions. The implications of these results are considered more fully in Chapter 8: Discussion.

In the next section, an analysis of the additional comments is discussed. This analysis provided a broader context and further support for the main constructs included in the research model.

\subsection{Additional Comments by Respondents}

In addition to providing empirical data to measure the research model, the survey also included one final (optional) question: Please share any other comments you may have concerning your organization's electronic recordkeeping system.

The response rate for this question was high. In total, $53.4 \%$ of the 193 active participants provided 103 comments. Many of the comments consisted of full essays, with one reaching 366 words in length. The resulting transcript provided observations, complaints, and suggestions.

Given the volume of the comments, an NVivo-based thematic analysis technique (introduced in Section 5.2.3, p. 84) was applied to the survey's additional comments transcript. Table 7.13 outlines the emergent themes and their relative frequencies as they were recorded by the participants. ${ }^{8}$

\footnotetext{
${ }^{8}$ Note that more than one thematic reference may be associated with a single respondent. In general, there is a 1:1 ratio of thematic reference to respondent; however, in the case of longer essay-like responses, there may be $2-3$ references per respondent. In no situation were there $>3$ references per respondent for a single theme.
} 
General praise for EDRMS implementation

Acknowledge value of electronic recordkeeping

19

$18.4 \%$

\section{General criticism for EDRMS implementation}

Lack of sufficient training

40

Search or classification problems 38

User interface difficulties $\quad 27$

Speed of operation $\quad 23$

Usage not organization-wide 22

$38.8 \%$

$36.9 \%$

$26.2 \%$

$22.3 \%$

Prefer alternative storage (e.g., shared drive) 20

$21.4 \%$

Security and/or access issues

$19.4 \%$

Poor technical support

$15.5 \%$

Unfavorable comparison to other systems

13

$12.6 \%$

EDRMS not required/don't create records

10

$9.7 \%$

Lack of use-enforcement policy

8

$7.8 \%$

Use is only due to social pressure

$6.8 \%$

Too busy to do recordkeeping

TOTAL

3

$2.9 \%$

2

$1.9 \%$

248

103 Responses

Table 7.13: Survey Open Answer Question: frequency of NVivo node-coding by number of reference quotations (References) and references as a percentage of the number of individuals (\% of Responses) completing this optional survey question. 
Nineteen respondents (18.4\%) included some positive comments concerning their EDRMS; however, those comments were often in the form of: "Electronic recordkeeping is great; but..." ${ }^{9}$ For example:

Electronic recordkeeping is necessary and can be a blessing, if the system is user-friendly and all staff have access to it. [Participant then proceeds to list aspects that are not user-friendly.]

Good to have documents all in one place, but takes a long time to save them.

I like the system and find it fairly easy to use. However, I know that some of my colleagues don't use it as they don't know how, or when they do, they have problems.

One of the most insightful comments reflected aspects of the perceived value of records construct as well as characteristics of trustworthy records. The following comment reflected the need for trustworthy organizational memory and highlighted the challenges of educating/motivating end users to input records in a manner that will enhance the value of this repository, rather than detract from its usefulness.

EDRMS is a tool well worthy of investment. The problem we have is around the quality control of documents (particularly versions and updates), naming conventions and the basic foundations of any process or system that make it easy to find the right information at the right time. There needs to be a tremendous amount of thought and education into how we want to make it successful for all to use without doubt that the information found is either inaccurate or out of date.

In addition, a large percentage of responses (38.8\%) highlighted a lack of sufficient training as a primary driver of usage problems. This is in spite of compulsory business unit training upon introduction, weekly EDRMS training sessions available for new users or those who require additional help, nominated local super-users, and online manuals illustrating the step-by-step basics. Nonetheless, three comments provided some insight into the need for buy-in and the importance of personal ownership/responsibility in learning to use the system:

\footnotetext{
${ }^{9}$ There were no comments that consisted solely of praise for their EDRMS. All comments included some caveats or criticisms.
} 
[N]ow that I have taken ownership of using [the system] as part of my everyday use, I find it a great tool.

I do not use it as I also do not see the point in it. As far as I am aware, it records documents and work, and the department already has a storage system for storing work and documents [i.e., a shared network-drive] so I use that instead [of the EDRMS]. I just don't see its point.

I strongly support the idea of an EDRMS, but sadly the organization has not really bought into [the system] and it is still only used in a few areas [...]. This, combined with a lack of senior champions, has done little to promote the use of [the system].

The next most frequent criticisms focused on the difficulty of search and/or classification problems (36.9\%) and user-interface difficulties (26.2\%). These were generally linked by the respondents to insufficient training and/or the poor quality of the recordkeeping metadata that is required to provide a useful foundation of content for Search function. For example, the name "draft.doc" would not be a suitably descriptive title for a record (nonetheless, it is very common), and while it might be expected that perhaps its location in context may help shed some light on the purpose of that draft document, another participant lamented that "many users have a single personal folder, crammed full of different records, and completely defeating the purpose of the system."

Finally, many participants ( $21.4 \%$ of comment responses) pointed out that although the EDRMS has been designated as the official records repository, it has not been made available to the entire organization.

It's only been partially rolled out across the organization. It was rolled out to our team over two years ago, and we have no option but to use it. However, none of the other teams we work with use it, which makes version control a nightmare when you're trying to work on a document with people from other teams. In this type of situation, trying to keep one master record in [the system] is an exercise in futility and eats up enormous amounts of time.

In general, the comments by the survey respondents provided additional support for the proposed research model. The subsequent thematic analysis further highlighted aspects of technology acceptance, the importance of 
organizational context, and differences in knowledge interpretation/perceived value of records. Furthermore, no additional factors were suggested that were not already covered by the existing research model. The complex combination of lack of buy-in and the poor understanding of the purpose of records, low quality recordkeeping metadata input by end users, and mixed social influence messages resulted in low-use of the technology. One interview participant concisely summarized the current situation:

As an information analyst, having good recordkeeping is a key to my performance. However, the use of [the system] in my area is appalling. I've done what what I could to improve things - I've trained, I've shamed, I've improved the structure, I've cleaned up records. But there's a disconnect. People have to use [the system], but nobody uses [the system]. There's just an unwillingness by most people to use it, and there's no lever to make people use it.

In summary, the optional comments by the participants at the end of the survey provided additional support for the current research model. More importantly, they provided a glimpse of the organizational context in which the electronic recordkeeping system is expected to be used. Furthermore, given the types of complaints, concerns, and grumbles, the comments also support the findings of the model in that perceived value of records (and primarily the regard for organizational memory) is a key driver for use.

\subsection{Chapter Summary}

In this chapter, the steps associated with the analysis of the survey data were discussed in detail. The response rate for the survey was quite high, with $76 \%$ of survey invitations resulting in a usable record - a total of 193 participant records - which was well in excess of the 60-120 samples estimated to be required for the analysis to be statistically valid. Demographic measures of the sample population were also described.

To further refine the measurement item pools, each construct pool was analyzed separately to determine its ability to reflect its parent construct. Using Principal Component Analysis (PCA) and a common graphical visualization called the Circle of Correlations, measurement items that were found to cluster were considered to be more uniform (unidimensional) in their capability to reflect their parent construct and were thus retained for use in the final model. Outliers (those items that did not cluster well) were considered in 
light of their performance in the card sorting activity and via the possibility of comprehension and interpretations differences between participants. Many of those measurement items were then excluded from the final model.

Partial Least Squares Path Modeling (PLS-PM) was then introduced as the preferred analysis technique for the survey dataset, with a short discussion of its relative merits. The PLS-PM analysis started with the outer (measurement) model, building on and validating the factor analysis results from the previous section. Once the outer model was deemed ready, it was statistically checked to make sure that its measures of unidimensionality were within optimal tolerances. This proved to be the case.

The focus then moved to the inner (structural) model, where the theoretical research model finally emerged for measurement. The PLS-PM path coefficients were calculated, showing significant promise for the new construct perceived value of records (which is discussed in more detail in Chapter 8).

Having effectively measured the structural model, the statistical significance of those measurements was determined by turning to standard regression model significance tests for the inner model, followed by the non-parametric bootstrapping validation technique to evaluate the overall precision of the PLS parameter estimates. The bootstrap findings validated the initial estimates completely.

Armed with a validated model, the research hypotheses were reviewed with an aim to determine how well the research model supported the hypothesized path relationships.

Finally, the survey's additional comments were analyzed, providing further contextual background for the interpretation of the research model while also adding support to the selected constructs and measurement items making up this model.

In the next chapter, Chapter 8: Discussion, the implications of the findings are considered and discussed. 


\section{Chapter 8}

\section{Discussion}

Contents

8.1 Chapter Overview . . . . . . . . . . 191

8.2 The Measures of the Constructs . . . . . . 192

8.3 The Research Model . . . . . . . . . . . 202

8.4 Chapter Summary . . . . . . . . . . 212

\subsection{Chapter Overview}

This chapter discusses the research findings reported in Chapter 7. First, the outer measurement model is examined, focusing on the measures of the constructs and the implications of the PCA-based clustering. The discussion then moves on to the interplay of the constructs by exploring the inner model (the research model) and considering the implications of how the empirical results affect the interpretation of the hypothesized effect paths. 


\subsection{The Measures of the Constructs}

The constructs were originally chosen to represent three conceptual aspects of the research model: technology acceptance, organizational context, and knowledge interpretation. The empirical results from Chapter 7 are discussed below, particularly as they apply to the measurement validity for each of the constructs.

\subsubsection{Technology Acceptance}

The two technology acceptance constructs used in this research were effort expectancy (perceived ease of use) and performance expectancy (perceived usefulness).

The original Technology Acceptance Model (Figure 2.9, p. 36) hypothesized that the cognitive responses (consisting of the above constructs) required moderation via attitude before having an effect on the behavioral response. However, subsequent models (e.g., TAM 2: Figure 2.10, p. 39 and TAM3: Figure 2.12, p. 43) dispensed with the attitude construct and instead linked the models' effect paths directly to the behavioral intention construct (which is, in this study, intention to use (ITU)). ${ }^{1}$

The measurement item pools were intentionally enlarged to include the measurement items used in the broader technology acceptance literature claimed by the UTAUT constructs as well as additional measurement items that emerged from the Stage 1 interviews. As discussed in Chapter 7, the circle of correlations greatly assisted in refining the final set of measurement items to represent a unidimensional (in the principal component space) measurement of each construct. In general, the technology acceptance literature's measurement items were found to be satisfactory and were mainly retained as measures for their respective constructs in the final research model analysis.

\section{Effort expectancy (perceived ease of use)}

On the surface, effort expectancy (EE) is one of the least complex of the constructs in the research model. It simply seeks to determine if a technology is easy to use. However, that apparent lack of complexity is itself a challenge

\footnotetext{
${ }^{1}$ The survey instrument captured measures for attitude in the unlikely event that the research model failed to provide significant path effects directly to intention to use. However, as confirmed in the later technology acceptance models (e.g., TAM2 and TAM3), attitude was not required to moderate those path effects in the model.
} 
for measurement. Many of the measurement items found in the literature could almost be considered tautological in their approach, with one common measurement item for perceived ease of use being: "I would find the system easy to use" (Davis, 1989; Davis et al., 1989).

To address this situation, the pool of EE measurement items was enlarged to include other contextual dimensions in which the effort of use could be measured in an electronic recordkeeping context. Examples include the ease of learning, the ease of filing, the ease of search, and the ease of records administration (see Table 5.5, p. 91, for the EE thematic codes that emerged from Stage 1).

Each of the emergent thematic codes contained within them a rich story of additional contextual complexity. For example, several of the Stage 1 interview participants expressed criticism of the content and quality of training available in their organizations. Some individuals subsequently explored the system interface and claimed to have successfully taught themselves, while others waited to receive training and then complained that it was inadequate. Those that didn't put effort into learning the system also reported that other measures of effort such as ease of filing or ease of search were also "difficult."

Nonetheless, the interviews indicated that additional training may not be the sole answer, as there were similar complaints from individuals who had attended training, but may not have actively engaged during that training. This issue is only raised here to indicate that all constructs and/or models are designed to simplify reality to a manageable and measurable level (BailerJones, 2003). In the case of this research, the measurement items were selected to best approximate the defined construct and were deemed appropriate when they were both logical and clustered in the circle of correlations.

The principal component clustering analysis resulted in a final (smaller) pool of measurement items (Table 7.1, p. 147). It should be noted that the exclusion of any measurement items from the final model was due primarily to their inability to cluster precisely enough with other such items. Having leftover measurement items does not suggest that there are additional constructs that need to be added to the research model to somehow explain these items. Nothing from the card sorting activities (particularly the open card-sorting) or the circle of correlations analyses suggested that there were any additional technology acceptance constructs lurking in the data. Instead, the excluded measurement items were simply not clear enough for the survey participants to uniformly interpret them in a similar manner. Retaining them would only have added error to the final analysis. 


\section{Performance expectancy (perceived usefulness)}

Performance expectancy (or perceived usefulness) has typically been the strongest of the technology acceptance constructs (Venkatesh \& Davis, 2000; Venkatesh \& Bala, 2008). In their research, Venkatesh and Davis (2000) found that "the performance expectancy construct within each individual model... is the strongest predictor of intention and remains significant at all points of measurement" (Venkatesh \& Davis, 2000, p. 447).

Once again, the pool of measurement items for performance expectancy was intentionally enlarged to include additional dimensions in which the performance/usefulness could be measured in an electronic recordkeeping context, while remaining true to the construct definition. See Table 5.2 (p. 87), for the thematic analysis from which these additional PE measurement items were sourced.

The circle of correlations was then applied to the survey data in an effort to identify the measurement items that clustered well. Performance expectancy's final measurement items are listed in Table 7.2 (p. 150). In general, the published construct measurement items were retained. The majority of the additional measurement items added on account of the thematic analysis were eventually excluded due to a lack of precision in reflecting only this construct. Those that were retained were unidimensional and tightly clustered in the circle of correlations.

However, usefulness is a very broad concept and the adopted construct definition is quite narrow. Does the technology acceptance literature's definition of performance expectancy/perceived usefulness continue to work in different cultural and/or use contexts? In the UTAUT model, performance expectancy was defined as

the degree to which an individual believes that using the system will help him or her to attain gains in job performance (Venkatesh et al., 2003, p. 447).

This definition focuses on personal gain and aligns usefulness with a competitive performance edge. Many of the published measurement items directly linked usefulness to increased job performance or even increased pay. This performance lens may work well in a competitive American corporate environment, where each employee will quickly adopt the technology that will provide them with a personal competitive advantage. This definition appears to celebrate individualism and, to some extent, self-promotion. 
In contrast, the culture of the New Zealand public sector and its public sector ethos (see p. 89) creates a very different environment in which usefulness can be defined. Many of the interviewees claimed no expectation of receiving personal recognition for their work, much less personal gain. Their altruistic public servant philosophy appears to seek motivation from sources other than gains in performance.

A dictionary entry for useful (Dictionary.com, 2014) is:

1. being of use or service; serving some purpose; advantageous, helpful, or of good effect: a useful member of society.

2. of practical use, as for doing work; producing material results; supplying common needs: the useful arts; useful work.

Perhaps there are other dimensions of perceived usefulness (and therefore other measurement items) that could replace Venkatesh and Davis' performance lens set. These new definitions and measures could reflect cultures that view usefulness through different lenses, and could thus provide a contextual definition of what usefulness means. Such a definition could be tailored to a culture and its use context.

Usefulness could be - helping out co-workers - streamlining policy development - improving the organization's ability to meet legislative requirements - making information discoverable to improve decision-making. All of these are other-centric and yet can reflect the goals of many public servants and still be covered by the definition of being useful.

In the case of electronic recordkeeping systems, the traditional performance expectancy measures that focused on attaining gains in job performance did not reflect the usefulness of a system that is mainly used for storage, reference, and compliance purposes. Far from being the "strongest predictor of intention", in the context of EDRMS, the effect of PE was found to be statistically insignificant.

\subsubsection{Organizational Context}

The bulk of recordkeeping theory finds its origins in Giddens' Structuration Theory (Section 2.3.1) and builds upon this theory to explain how records and their requirements reflect and adapt to the changing needs of society. Because the adoption of electronic recordkeeping systems also occurs within the context of a society, the organizational context constructs were selected to reflect the three proposed structurational interactions that form a society, namely 
communication, power, and sanction. Social influence (SI), which is primarily based on the subjective norm, was selected to reflect the communication and to some extent, the sanction interactions. Perceived power security (PPS) was selected to reflect Giddens' power interaction.

The outcome of the analysis in Chapter 7 showed clear clustering of the combined SI and PPS measurement items into three distinct groupings. See Figure 7.5 (p. 152) for the SI circle of correlations and Figure 7.7 (p. 156) for the SI and PPS combined analysis. Further consideration of the three clusters revealed a strong alignment with Giddens' structurational interactions.

\section{Social Influence}

The original social influence (SI) measurement items (Table 5.10, p. 97) were drawn from the literature in order to represent UTAUT's broader social influence construct and its source constructs. Unlike technology acceptance measures, the literature's SI measurement items were found to cover all of the emergent interview themes (Table 5.8, p. 94). No additional dimensional measurement items were added as a result of the Stage 1 interviews, although some items were slightly adapted to the recordkeeping context.

In the UTAUT model, Venkatesh et al. (2003) found that "none of the social influence constructs [were] significant in voluntary contexts; however, each becomes significant when use is mandated" (Venkatesh et al., 2003, pp. 451-452). In this research, whether the use of their electronic recordkeeping system is voluntary or mandated was not well understood by the participant sample. Table 8.1 shows the mixed responses, with $60 \%$ replying yes and $40 \%$ either replying no or not sure. Given the lack of clarity and the apparent lack of organizational policy on recordkeeping in the sample organization, measuring the potential impact of mandatory versus voluntary use on social influence was not possible. However, the effect on intention when

Is use of the system mandatory?

\begin{tabular}{ccc}
\hline Stated Belief & No. of Participants & Percentage \\
\hline Yes & 116 & $60.1 \%$ \\
No & 41 & $21.2 \%$ \\
Not Sure & 36 & $18.7 \%$ \\
\hline
\end{tabular}

Table 8.1: Number of survey respondents who believed that use of the electronic recordkeeping system was mandatory in their organization. 
recordkeeping is clearly understood to be mandated would be a candidate for future research.

While discussing their statistically insignificant findings, Venkatesh et al. (2003, p. 452) acknowledged that "social influence in technology decisions is complex" and then suggested that social influence has an impact via three mechanisms. The proposed mechanisms (compliance, internalization, and identification) are based on Warshaw's (1980) work, and predate Giddens' (1984) Structuration Theory.

In this research, there were three distinct groups or clusters of SI items detected. These groups were interpreted as aligning with the three structurational interactions (communication, power, and sanction). To further test the structurational interpretation (versus Warshaw's interpretation), the perceived power security items were added to the circle of correlations analysis. This additional analysis revealed a clustering with the Giddens' power interaction group. In other words, the power measurement items shared a similar principal component orientation to the social influence power grouping (Figure 7.7, p. 156).

There are two implications of this interpretation. First, the social influence construct is multidimensional. ${ }^{2}$ In other words, if each of the three SI clusters had been treated as separate constructs in the measurement model, they would have resulted in different path coefficients and significance levels. Second, this multidimensionality can be interpreted well via the structures of society put forward by Giddens' Structuration Theory, where social influence could be redefined in terms of the structurational interactions:

$$
\text { social influence }=\{\text { communication, power, sanction }\}
$$

In an effort to retain a unidimensional construct, only the measurement items that aligned with Giddens' communication interaction were retained to represent the SI construct. This pool of measurement items includes the measurement items used in UTAUT to represent SI and were a similar pool to those used to represent the original subjective norm.

The final measures of SI (now defined in structurational terms as $S I_{\text {comms }}$ to reflect their measure of the communication interaction) were purged of any

\footnotetext{
${ }^{2}$ Although the UTAUT model claims that social influence unites multiple overlapping constructs, combining the measurement item pools resulted in three PCA clusters. This analysis suggests that social influence, as claimed in UTAUT, is not unidimensional. However, the final UTAUT measurement items selected to represent SI correspond only with the communication interaction cluster. Thus, $S I_{\text {comms }}$ has been confirmed by this analysis to be unidimensional.
} 
measures relating to sanction. As sanction was not included in the original model (and no effort was made to refine a pool of sanction measurement items prior to the survey), the inclusion of Giddens' sanction interaction was necessarily out of scope. However, research into defining and measuring the effect of sanction behaviors on technology acceptance would be a candidate for future exploration.

\section{Perceived Power Security}

Perceived power security (PPS) was selected from the literature as the construct that was most likely to represent Giddens' final social interaction: power. As discussed in Section 5.2.4 (p. 98), many of the interview participants did not believe that power (either represented as allocative or authoritative) was likely to affect the intention to use an electronic recordkeeping system. In addition, the measurement items taken from the literature had proved to be problematic. The original wording of the measurement items had been confusing to participants in the proofreading stage, and subsequent efforts to create meaningful measurement items had shown mixed results in the card-sorting stage (Figure 5.5, p. 126).

Nonetheless, these measurement items were partially successful. When they were added to the social influence items in the circle of correlations analysis (see discussion above and Figure 7.7, p. 156), their shared orientation with other power-associated measurement items provided confirmation of their PCA power alignment and appeared to hold much promise for the research model.

However, the observed PCA clustering was primarily due to the scale of differences between the power grouping and the other SI groups. A more restricted circle of correlations analysis focused solely on the combined pool of power measurement items (Figure 7.8, p. 157) revealed that the PPS constructs were not clustering as well as the $S I_{\text {power }}$ constructs. In order to retain a unidimensional measure of power, all of the PPS measurement items were eventually dropped from the model. To represent the change in measurement items, the PPS label was changed to POW (to represent the power interaction, but separate it from the PPS measurement items). The new POW construct retained the same construct definition as PPS, but uses different measurement items. Nonetheless, the logic and predicted path effects of POW on the inner research model remained unchanged.

The role of structuration theory in defining organizational context has the potential to provide a powerful lens for empirically viewing the cultural 
aspects of technology acceptance. Additional investigation into developing and defining measurement constructs based on the structurational interactions would be another candidate for future research. However, incorporation of Giddens' structures and modalities (see Figure 2.2, p. 20) into this future research could provide even more insight into organizational context. In a study of information culture, Zheng (2005) argued that the development of a mature information culture is the key to increased use of technology and used Giddens' modalities as a basis for proposing a mechanism for actioning that change. Additional research into information culture and its relationship with recordkeeping is ongoing (Oliver \& Foscarini, 2014).

\subsubsection{Knowledge Interpretation}

The final conceptual aspect of the research model focused on the knowledge interpretation aspect of electronic records and process of recordkeeping. As a suitable validated construct was not found in the literature, a new construct was developed out of Schultze and Stabell (2004)'s knowledge management discourses (Section 2.5.2, p. 35). These were used to provide a foundation for discussing the value of records and the perceived value of the information they contain.

Schultze and Stabell (2004)'s discourses suggested how knowledge (or in this case, recorded evidence) can be viewed from the perspective of a social order continuum (consensus to dissensus) and an epistemological continuum (dualism to duality). The epistemological continuum aligned with Giddens' approach to Structuration Theory (and his attempt to reconcile the two), and thereby linked the discourses to the common structuration-based theoretical platform used throughout this research. The discourses lens also provided the basis for the Stage 1 interview questions and the development of the new construct, the perceived value of records.

\section{Perceived Value of Records}

The subsequent analysis of the interviews provided a set of thematic codes (Figure 5.14, p. 101). When they were considered in light of Upward's Records Continuum Model (Section 2.3.2, p. 21), the codes appeared to group well according to Upward's continuum of evidentiality. The continuum of evidentiality consists of four dimensions: trace, evidence, corporate/individual memory, and collective memory. Semantically, the emergent measurement 
items appeared be grouped into three groups (where memory uses were combined into a single category):

- The ownership of accountability corresponds to the trace dimension of the records continuum. It represents the role that records play to trace accountability and the responsibility that users take in maintaining this aspect of recordkeeping.

- The need for trustworthy evidence reflects the evidence dimension of the records continuum. It also represents the importance of evidence, such as the importance of keeping a record of one's personal work as evidence of job quality.

- The value of organizational memory corresponds to the two memory dimensions of the records continuum. It represents the organization's ability to "collect, store and use knowledge" (Olivera, 2000, p. 811) and reflects the value of the content of those records and whether the recorded knowledge would have value in the future.

However, when these measurement items were analyzed using PCA, the circle of correlations only provided two clusters rather than three (Figure 7.9 and Table 7.4). Group 1 aligned well with the organizational memory dimension and reflected the need and importance of records as a vehicle for capturing information for use in the future. Unfortunately, Group 2 was not as logically consistent, and did not reflect the record continuum's trace or evidence dimensions well, nor did it semantically reflect the accountability or trustworthy evidence groupings as suggested above. Consequently, the measurement items in Group 2 were dropped from the measurement model.

Nonetheless, the two groups that emerged from the PCA circle of correlations did suggest that the perceived value of records may consist of two dimensions. As mentioned above, Group 1 appeared to reflect the value accorded to the content of the records. As noted earlier, a document is defined as "recorded information... which can be treated as a unit" (ISO 15489.1, 2002 , p. 3). Documents can be (and are) a vehicle for storing organizational memory, and many public sector organizations rely on shared-drives or other ad hoc locations to store these documents for use in the future. They value the content of those documents.

Group 2 provided a hint of what the second dimension may be. Documents cannot be records unless they are under systematic control. As defined in ISO 15489: Records Management, a record is "information created, received, 
and maintained as evidence and information by an organization or person, in pursuance of legal obligations or in the transaction of business" (ISO 15489.1, 2002 , p. 3), while records management is defined as the "field of management responsible for the efficient and systematic control of the creation, receipt, maintenance, use and disposition of records, including processes for capturing and maintaining evidence of and information about business activities and transactions in the form of records"(ISO 15489.1, 2002, p. 3).

Therefore, it is likely that the second dimension reflects the evidential importance and contextual tracking of a document; in other words, the value of its context. Information about its context is held in its recordkeeping metadata and in the systems that support electronic recordkeeping. In terms of the trace and evidence dimensions of the records continuum, it is the recordkeeping metadata that can answer such questions as: what is the status of this draft policy? (with the metadata allowing users to trace their record in the system), or: did this policy decision predate that action? (which relies on the metadata to provide trustworthy evidence of timing). Even questions such as, Who last edited this record? is information stored in the metadata rather than in the document itself. This research, then, suggests two dimensions that are expected to contribute to the perceived value of records:

$$
\text { perceived value of records }=\text { value }\{\text { content }, \text { context }\}
$$

The perceived value of records was initially defined on p. 63 as a user's belief that a document has value beyond the current application and is worthy of storing for the future. This definition can now be updated as follows:

Perceived Value of Records: the perceived importance that a user places on a document's information content combined with the importance accorded to its evidential context through time. (Lewellen, Hooper, \& Oliver, 2014a)

Identifying the content and context dimensions of a record provides an approach to explaining the current state of electronic recordkeeping systems adoption within public sector organizations, as well as explaining the resistance to moving away from legacy shared-drive systems in favor of an EDRMS.

Table 8.2 illustrates a hypothetical matrix consisting of the perceived value of content (organizational memory) [low-high] and the perceived value of information about a document's context (recordkeeping metadata) [low-high]. 


\section{Content}

\begin{tabular}{|c|c|c|c|}
\hline & & Low & High \\
\hline & & System: workflow & System: $E D R M S$ \\
\hline & & Solution is only required & Records have need for both \\
\hline & Hior & to track documents. The & informational and eviden- \\
\hline & 1119ा1 & content of documents has & tial characteristics to be \\
\hline & & little future value. & systematically managed. \\
\hline Context & & Cost: medium & Cost: high \\
\hline & & System: ad hoc & System: shared-drives \\
\hline & & Documents have little or & Content can be shared \\
\hline & Low & no evidential value and no & with simple shared drives, \\
\hline & & $\begin{array}{l}\text { tracking is required. } \\
\text { Cost: low }\end{array}$ & $\begin{array}{l}\text { wikis or intranet. } \\
\text { Cost: medium }\end{array}$ \\
\hline
\end{tabular}

Table 8.2: The perceived value of records suggests four system requirement categories based on the perceived need of content (organizational memory) versus context (recordkeeping metadata).

The matrix suggests a logical approach to information management (and the associated technology solution) that would meet those perceived needs.

This research has focused on developing and measuring the content dimension of the perceived value of records. Future research to develop a construct representing the context dimension could provide additional insight into how organizations value their information as well as explaining or predicting the choices they make in managing that information.

In the next section, the inner (structural) model will be discussed, along with the implications of the hypothesized path effects and interactions.

\subsection{The Research Model}

The research model consisted of nine hypothesized path effects linking the six research constructs. The path coefficients are graphically portrayed by location in Figure 7.12 (p. 173) and by magnitude in Figure 8.1 (below). The empirical results for each of the hypothesized effects are summarized in Section 7.5 (p. 181). In this section, each of the hypothesized path effects and the implications of the measurement results are discussed. 


\subsubsection{Hypothesized Path Effects}

The research model hypothesized nine path effects, five of which impacted the intention to use (ITU) directly. Figure 8.1 illustrates the total magnitude of those path effects on ITU by combining the direct effects (where the construct impacts ITU directly) and indirect effects (where a construct impacts a construct that impacts ITU).

\section{Bar chart of the path effects on Intention to Use \\ $\square$ Indirect $\square$ Direct}
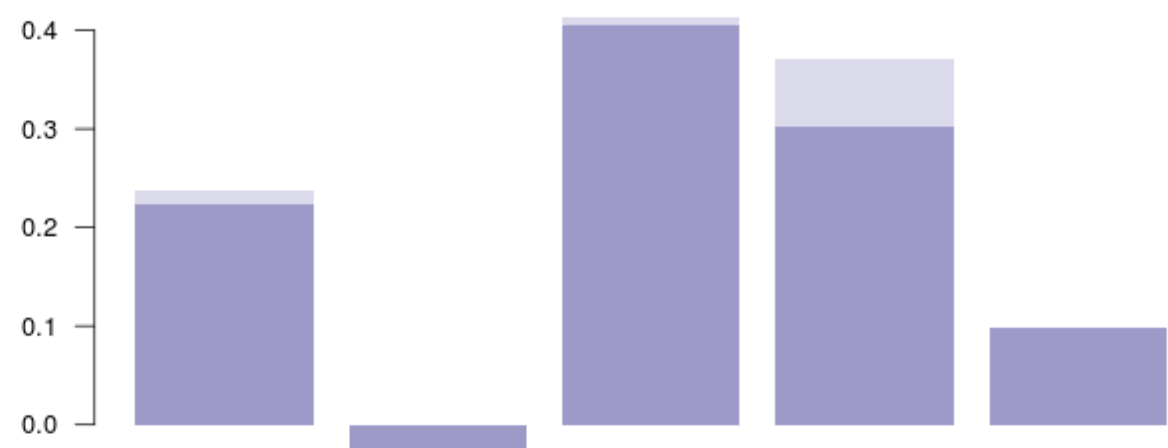

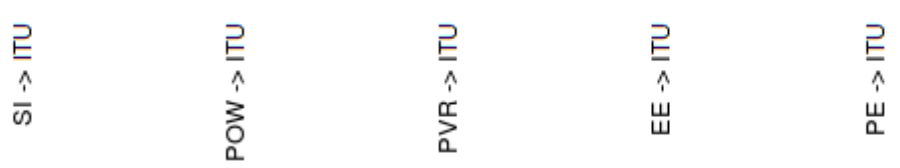

Figure 8.1: Bar chart of the path effects on Intention to Use (ITU)

\section{Technology Acceptance}

Effort Expectancy The first two hypotheses proposed a direct effect of effort expectancy (EE) on intention to use (ITU), as well as an indirect effect of EE on ITU via performance expectancy (PE). The Chapter 7 analysis found that the path coefficient of the EE construct was both strong and statistically significant. Both of these hypotheses were supported.

- Hypothesis $\mathbf{1}$ [EE $\rightarrow I T U]$ : effort expectancy (perceived ease of use) will have a positive effect on a user's intention to use an electronic recordkeeping system.

\section{Supported}


- Hypothesis $2[E E \rightarrow P E]$ : effort expectancy (perceived ease of use) will have a positive effect on a user's performance expectancy (perceived usefulness) of an electronic recordkeeping system.

\section{Supported}

As seen in Figure 8.1, the $E E \rightarrow I T U$ total effect was measured at 0.37 (consisting of a direct effect of 0.30 and an indirect effect via PE of an additional 0.07). Effort expectancy represents the second most important construct in the research model.

Given the importance of EE in explaining ITU in the research model, it was interesting to see that the raw effort expectancy results (see Figure E.1, p. 258) showed that the majority of the survey participants did not find the technology easy to use. This apparent contradiction was later resolved when the relationship between the variables was viewed as a correlation matrix (see the interaction between EE and ITU in Figure 7.13, p. 178). This matrix indicated that some people who find it difficult to use will use it anyway, whereas all people who find it easy to use will use it. There is a definite positive correlation between the EE and ITU constructs. In other words, the intention to use the system increases as people find it easier to use.

The strength of effort expectancy's effect in the model would suggest that any strategy that reduces the effort required of end users would dramatically increase use. For example, many of the recorded complaints were targeted at the effort associated with the need to enter metadata (as compared to using a shared-drive with no metadata requirements). The overhead associated with manually entering metadata is a known barrier to the use of electronic recordkeeping systems (Joseph et al., 2012; Kettunen \& Henttonen, 2010; Polfreman \& Rajbhandari, 2008). Therefore, any efforts that can reduce or automate the metadata entry requirements are likely to have the greatest impact.

Another approach would be to increase the awareness and importance of context information to end users as discussed on p. 202. If end users understand and value the contextual information that electronic recordkeeping can provide, then the need for that contextual information may exceed the annoyance barrier associated with having to enter that metadata. However, a two-pronged approach, consisting of increasing the demand for and use of contextual information while simultaneously reducing/automating much of the metadata entry requirements, would be expected to provide the greatest impact on the intention to use associated with effort expectancy. 
Performance Expectancy As discussed earlier, performance expectancy (or perceived usefulness) has generally been the strongest of the two main technology acceptance constructs. In their work on TAM2, Venkatesh and Davis (2000) emphasized this construct's importance: "Across the many empirical tests of TAM, perceived usefulness has consistently been a strong determinant of usage intentions, with standardized regression coefficients typically around 0.6" (Venkatesh \& Davis, 2000, p. 187). In the later UTAUT model, the "performance expectancy construct within each individual model [was found to be] the strongest predictor of intention and remains significant at all points of measurement" (Venkatesh \& Davis, 2000, p. 447).

Nonetheless, in the context of electronic recordkeeping systems, this research appears to reflect an exception to the rule. The impact of $P E \rightarrow I T U$ was measured as having a small positive path coefficient of 0.098 (as compared with Venkatesh \& Davis' impressive 0.6). In addition, it was statistically insignificant, meaning that it may have no effect at all insofar as technology solutions used in a recordkeeping context are concerned.

- Hypothesis 3 [PE $\rightarrow I T U]$ : performance expectancy (perceived usefulness) will have a positive effect on a user's intention to use an electronic recordkeeping system.

\section{Not Supported}

As mentioned in Section 8.2.1, p. 194, the effect strength of PE was expected to be less for recordkeeping technologies in the context of the public service. This has to do with the performance lens associated with the published PE measurement items. The performance emphasis misses the contextual mark in two ways.

First, electronic recordkeeping systems are not focused directly on job performance improvements. It is true that the competent use of an EDRMS can have a positive effect on job outcomes by providing consistent and reliable storage and improved search and access to historical records that may improve decision-making; however, the primary use of electronic recordkeeping is for storage, reference and compliance purposes. The link back to improved job performance is weak.

Second, as mentioned on p. 89 and in Section 8.2.1, p. 195, many of the interviewees displayed a public sector ethos and claimed no expectation of receiving personal recognition for their work, much less personal gain. Their altruistic public servant philosophy appears to seek motivation from sources other than from personal gain. 
In cases where a new technology may give a user a competitive edge, this construct is expected to continue to weigh heavily (and significantly) in their decision to use that technology. However, in the case of electronic recordkeeping, the use of the system is less likely to be perceived to help the individual user in this manner. In contrast, use will help others and provide accountability and transparency at the organizational level, but the performance edge provided by good recordkeeping to the individual user is expected to be perceived as small.

In this case, the technology acceptance constructs behave in a different manner to the cases presented in the literature. Based on the technology acceptance constructs, users appear to be willing to use this technology only if the system is easy to use. Furthermore, they see very little usefulness in the system in terms of their job performance, and what they do see does not significantly affect their intention to use the system.

As proposed in the research model, other factors must be considered to explain the intention to use an EDRMS.

\section{Organizational Context}

The organizational context constructs include social influence and perceived power security. Social influence (SI) represents the structurational interaction of communication $\left(S I_{\text {comms }}\right.$ ), whereas perceived power security (POW) represents the structurational interaction of power (and consists of the $S I_{\text {power }}$ measurement items).

Social Influence As seen in Figure 8.1, the $S I \rightarrow I T U$ total effect was measured at 0.24 (consisting of a direct effect of 0.22 and an indirect effect via PE of an additional 0.016). Social influence represents the third most important construct in the research model.

- Research Hypothesis $4[S I \rightarrow P E]$ : social influence will have a positive effect on a user's performance expectancy (perceived usefulness) of an electronic recordkeeping system.

\section{Supported $^{3}$}

\footnotetext{
${ }^{3}$ The effect of $S I \rightarrow P E$ was statistically significant; however, the effect of $P E \rightarrow I T U$ was not. As such, the inclusion of the indirect effect for SI provides an indication of the path coefficient strength, but not the statistical validity.
} 
- Research Hypothesis 5 [SI $\rightarrow I T U]$ : social influence will have a positive effect on a user's intention-to-use an electronic recordkeeping system.

\section{Supported}

As discussed in Section 8.2.2, p. 196, Venkatesh et al. (2003) observed that SI was only significant in mandatory use situations. In the sample organization, it was not clear among the survey participants whether use of the recordkeeping system was mandatory or not. Nonetheless, the effect of $S I \rightarrow I T U$ was both strong and highly significant. Lacking clear organizational recordkeeping guidelines in the sample organization, it would appear that the 'opinions of important others' provided the required use guidance in the absence of clear written policies. This suggests there may be other aspects of operational policy that are left to grow organically, and that staff may rely on their peers and managers to communicate acceptable norms and behaviors. If this is the case, then the relevance of structuration theory in explaining technology acceptance increases as the societal norms concerning usage behaviors recursively evolve in the absence of a static policy.

A clear policy mandating the use of an EDRMS (supported by a culture that respects and adheres to clear policies) would be expected to reduce the importance of social influence in the research model. In other words, users would know what they were expected to do and would rely less on the opinions of others. However, this outcome is the opposite of what was found by Venkatesh et al. (2003), who said "none of the social influence constructs are significant in voluntary contexts; however, each becomes significant when use is mandated" (Venkatesh et al., 2003, pp. 451-452).

This apparent conflict can be explained by analyzing the channel by which an organization's policy expectations are transmitted. If a written mandatory use policy is communicated to users via managers and peers, then social influence will be seen as being a significant factor in use. However, if the written mandatory use policy is known via a non-social channel (e.g., as part of the code of conduct or via one's job description and performance evaluation criteria), then a social communication channel as a mode of transmission would be expected to be less important. In the case of clear written policies transmitted via a non-social channel, it is likely that Giddens' communication interaction would decrease in importance in the research model, while Giddens' sanction interaction would increase as managers and peers increasingly apply negative social pressure against non-users. 
Future research is required to determine the relevance of communication and sanction social interactions within mandatory use situations, taking into account cultural context and mode of transmission of these policies. It is expected that a campaign to present clear policies and expectations would effectively reduce the need for social communication interactions, while increasing the role of sanction interactions as a means to boost compliance.

Perceived Power Security The remaining organizational context construct in the research model is perceived power security (POW). Its hypothesized path effects are listed below.

- Research Hypothesis 6 [POW $\rightarrow$ ITU]: perceived power security will have a positive effect on a user's intention-to-use an electronic recordkeeping system.

\section{Not Supported}

- Research Hypothesis $7[P O W \rightarrow E E]$ : perceived power security will have a positive effect on a user's effort expectancy (perceived ease of use) of an electronic recordkeeping system.

\section{Not Supported}

The impact of $P O W \rightarrow I T U$ appeared to be small and negative; however, it was also statistically insignificant. So, the logical interpretation is that it had no impact at all in the model.

This observation is further supported by comments that emerged in the stage 1 interviews (p. 98). When pressed in the interviews, the participants acknowledged that someone could conceivably feel a loss of power associated with storing records in a common system; however, the majority of participants remained unconvinced as to the importance of power, power-security, or powerloss in explaining system use.

The measurement model supports this sentiment. Nonetheless, there remains the potential for records to be transformed into a source of power. As discussed earlier, two kinds of power can be manifested: authoritative extending over people, and allocative - extending over objects or materials (Orlikowski, 1992, p. 405).

As use of electronic recordkeeping becomes more mature (and the awareness of the value of context and access controls increases), it is possible that individuals will begin to use access rights or contextual storage as means of expressing power over others. Most EDRMS prevent unapproved destruction 
of records; however, access rights are generally assigned to a record type or classification, and the selection of that classification (along with granular access controls) is left to the individual.

Record storage and access thus have the potential to be used maliciously. For example, placing a record in a different context could make that record appear to be something that it is not, and opening access to that record too broadly could damage someone's reputation. Alternatively, reducing access to or hiding records could similarly affect decisions and reflect power agendas.

In addition, should records grow in importance and become a form of measurement (for example, linking pay to records of one's work), it is possible that individuals could enter metadata in such a way that the measures would load positively onto themselves or perhaps load negatively onto others. In this way, power could become a significant factor in the research model.

However, the evidence suggests that recordkeeping systems have not (yet) been used for these sorts of power struggles. Nonetheless, future researchers could consider revisiting this construct, especially in organizations that display aspects of dissensus in their organizational culture.

\section{Knowledge Interpretation}

The final conceptual aspect of the research model references how individuals interpret the value of knowledge. As discussed in Section 8.2.3, the new construct perceived value of records (PVR) captures the value associated with organizational memory (content), but excludes measures of contextual value.

Perceived Value of Records As seen in Figure 8.1 (p. 203), the total effect of $P V R \rightarrow I T U$ was measured with a path coefficient of 0.41 (consisting of a direct effect of 0.406 and a small indirect effect via PE of an additional 0.007). ${ }^{4}$ Perceived value of records represents the most important construct in the research model.

The related research hypotheses are included below.

- Research Hypothesis $8[P V R \rightarrow P E]$ : perceived value of records will have a positive effect on a user's performance expectancy (perceived usefulness) of an electronic recordkeeping system.

\section{Not Supported}

\footnotetext{
${ }^{4}$ The effect of $P V R \rightarrow P E$ was not statistically significant, nor was the effect of $P E \rightarrow I T U$. Thus, the inclusion of the indirect effect for PVR provides an indication of the path coefficient strength, but not the statistical validity.
} 
- Research Hypothesis 9 [PVR $\rightarrow$ ITU]: perceived value of records will have a positive effect on a user's intention-to-use an electronic recordkeeping system.

\section{Supported}

Research Hypothesis 8 proposes that individuals with a high perceived value of records will be more likely to believe that an EDRMS is a useful tool that will further increase their job performance. As discussed earlier, performance expectancy was found to have an insignificant role to play in the context of electronic recordkeeping. The reduced importance of PE was attributed to the performance lens of the PE construct's definition of usefulness.

In other words, the research finding suggests that survey participants saw little or no impact of using a recordkeeping technology on their job performance. With this perception in mind, further attempts to create a logical connection between perceived value of records and job performance would no longer appear likely or logical. The results are in alignment with this interpretation. The indirect path coefficient was extremely small (near zero) and was also statistically insignificant.

However, Research Hypothesis 9, which posited a direct effect of perceived value of records on the intention to use a recordkeeping system, was strongly supported with a high level of statistical significance. It is perhaps unsurprising that individuals who generally value records are likely to use an electronic recordkeeping system. However, the strength of the direct $P V R \rightarrow I T U$ effect has an important implication in identifying the epistemological perspective of the survey participants, and may provide some insight into the cultural norms of the research population.

As noted earlier, Schultze and Stabell (2004)'s four discourses for knowledge management research (see Figure 2.16 on p. 52) illustrate different world views concerning the value of knowledge in differing social contexts. The literature suggests that the main difference in the epistemological perspectives is the perceived value-duration of a particular knowledge artifact. Those adhering to a neo-functionalist or critical knowledge discourse see all records as potential long-term assets that are worthy of keeping and managing for use in the future. In contrast, those adhering to a constructivist or dialogic knowledge discourse perceive the same knowledge artifacts as merely supporting the current working knowledge and conclude that these same knowledge artifacts have only short-term value. These latter individuals would see little 
or no reason to invest in the effort to formally classify and manage records beyond their current application.

The strength of the $P V R \rightarrow I T U$ effect provides strong evidence for the prevalence of dualism as the epistemological world view for the majority of the survey participants. These individuals regard documents and records as assets that have value beyond today's working knowledge. According to this view, records have the potential to have value in the future and in new and different contexts. This view is also in alignment with Upward's Records Continuum Model (Section 2.3.2, p. 21), which is built on the tacit assumption of dualism and records-as-assets that require management.

The prevelance of dualism is further supported by the Stage 1 interviews, where several participants spoke of taking pride in being part of the organizational record, and one participant went so far as to require that his staff not use common acronyms so that distant future readers of their reports would have greater clarity as to their contextual meaning.

In addition, the insignificant impact of power in the research model provides insight into the social order dimensions of Schultze and Stabell (2004)'s knowledge management discourses. The surveyed organization had displayed anecdotal evidence of dissensus, with participants referring to tension between business units as well as between senior management and workers. However, the perceived context of the use of electronic recordkeeping for the majority of users appears to be at a more local level. The lack of power politics combined with a high reliance on social influence for guidance on social norms and expectations (pertaining to the use of their EDRMS) provides evidence of a consensus-based social order, at least at the local level.

The presence of a consensus social order in a population that has adopted dualism as their epistemological frame of reference neatly places the majority of the sample into the knowledge discourse of the neo-functionalists. As discussed earlier, the neo-functionalists are the most likely group to adopt electronic recordkeeping. Their metaphor of knowledge is an asset, and the role of knowledge in the organization is "progressive enlightenment, prediction, reduction of uncertainty, and optimal allocation of resources" (Schultze \& Stabell, 2004, p. 556).

The neo-functionalist world view is the foundation on which a positive information culture can be built. Its presence bodes well for the future of electronic recordkeeping as a distributed responsibility. Although this research has detected the presence of neo-functionalist thinking, it has not quantified the distribution of people among the four discourses. Future research into identifying and measuring the distribution of people among the knowledge 
discourses could provide additional insight where electronic recordkeeping systems adoption has not gone as planned.

\subsubsection{The Explanatory Power of the Model}

The most important constructs in the research model (in rank order) were the perceived value of records, effort expectancy, and social influence. The model confirms that the acceptance and use of an electronic recordkeeping system requires that its users have a high perceived value of records. In this case, the users with the most positive attitude toward recordkeeping have an epistemological bias in favor of knowledge as a dualism and generally function within a social order based on consensus. The importance of the social order is illustrated by the strong effect of social influence in the model.

The research model also provides insight into the reported challenges that have been experienced concerning the acceptance and uptake of electronic recordkeeping systems. In the case of EDRMS, the research model revealed that the performance expectancy/perceived usefulness construct fails to pull its weight in affecting system use. It is likely that change management strategies appealing to the usefulness of this class of system will miss the mark with users. This leaves effort expectancy as the sole remaining technology acceptance construct, and due to the nature of recordkeeping metadata, the implementation of these systems can be quite complex.

Overall, the research model demonstrated moderate to high explanatory capability, with the combined research constructs explaining more than half of the observed variance associated with the intention to use the electronic recordkeeping system $\left(R^{2}=0.52, G o F=0.50\right)$.

\subsection{Chapter Summary}

This chapter discussed the findings of the research and provided an interpretation of their significance. First, the outer (measurement) model was explored. The conceptual research model was used as a framework to introduce and consider each construct in context. The findings from the various stages of the research were drawn together to provide insight into each construct and a means to interpret each of its dimensions and its role in the research model. In several cases, the research findings identified additional theoretical dimensions that may produce new avenues for future research. 
Next, the hypothesized interactions of the constructs were discussed, with an emphasis on the levels of support provided to each of the nine research hypotheses that formed the inner (research) model. Again, the findings from the various stages of the research were drawn together to provide insight and interpretation for each instance whether a hypothesis was supported or not supported.

The research model produced moderate to high explanatory capability. However, the pattern of non-support associated with one of the technology acceptance constructs shed additional light on the importance of organizational context and knowledge interpretation in the acceptance and use of electronic recordkeeping systems. 


\section{Chapter 9}

\section{Conclusions}

\section{Contents}

9.1 Chapter Overview . . . . . . . . . . 215

9.2 Overview of the Research . . . . . . . 216

9.3 Contributions of the Research . . . . . . . 221

9.4 Limitations of the Research . . . . . . . . 224

9.5 Directions for Future Research . . . . . . 227

9.6 Chapter Summary . . . . . . . . . . . 230

\subsection{Chapter Overview}

In this final chapter, the research is summarized and brought to a close. Firstly, the research process, as it was conducted, is presented. Next, the contributions of the research are identified and discussed, including the theoretical benefits to academics and the pragmatic implications for practitioners and managers. The limitations of the research are then examined. These limitations pertain to the conceptual model, the research design, and the research instrument. Finally, directions for future research are outlined. 


\subsection{Overview of the Research}

This section will briefly summarize the research, the conceptual model, the research design, and the findings.

\subsubsection{Research Gap}

The discipline of recordkeeping has long been maintained by professional records managers. However, with the introduction of electronic recordkeeping systems, the responsibility for recordkeeping has moved from the records professional to the creators of the record - the end users (Joseph et al., 2012).

Many large-scale implementations of organization-wide electronic document and records management systems (EDRMS) have met with limited success and acceptance. However, as a class, the EDRMS had not yet been investigated empirically using the validated technology acceptance models. In addition, the EDRMS as a foundational organizational technology plays a unique role in that its primary focus is generally ancillary to the jobs and responsibilities of its primary users.

Given that these end users are now responsible for the creation of the majority of an organization's records (and many choose not to participate), the research question that sought to further the understanding in this area was:

- What factors influence a user's intention to use an electronic recordkeeping system?

\subsubsection{Research Objectives and Conceptual Model}

Converted into the research objectives, the aim of the research was:

- To identify the factors that influence a user's intention to use an electronic recordkeeping system.

- To determine the relative importance of the factors that influence a user's intention to use an electronic recordkeeping system.

The literature review sought to more fully understand the role of records and recordkeeping in society. Records and recordkeeping were defined and considered in light of the key characteristics of records, namely: authenticity, reliability, integrity, and usability (ISO 15489.1, 2002, p. 7). A short history 
of how these key characteristics have been used to create and manage records helped to place the EDRMS' current design and manifestation in context.

One of the primary functions of records is to act as evidence to demonstrate that a business function or transaction has occurred. As such, recordkeeping primarily relies on social theories such as Giddens' (1984) Structuration Theory to understand how society conceives of that evidential threshold, and how that threshold can change over time and space, as explained by Upward's (1997) Records Continuum model. These theories influenced the development of national and international standards which, when combined with work on recordkeeping metadata models, strongly influenced the development of the EDRMS in its current form.

In developing the conceptual research model, the technology acceptance literature was used as the model's core with system use being the dependent variable. As the EDRMS could be seen as having multiple potential uses (e.g., search, retrieval, security, workflow, etc.), the research focused on the point of contribution. All other uses require that records first be entered into the system.

The conceptual model was extended to include the organizational context. Records and the information they contain are necessarily created within the context of an organization. The organization is a microcosm of society. The social interactions that form that society, as put forward in Giddens' Structuration Theory, were expected to effect how people viewed the records and recordkeeping system within that society.

In addition, the literature also suggested that individuals view information differently, giving rise to the third part of the conceptual research model: knowledge interpretation. The knowledge management literature suggested several approaches. Schultze and Stabell (2004) provided the four discourses of knowledge management research combining a social order dimension (consensus to dissensus) that reflects the hegemonic order within the organizational context with an epistemological dimension that mirrors that used by Structuration Theory (dualism versus duality). The resulting matrix suggested that only one of the four discourses was conducive to successful information management and recordkeeping. In other words, how one views recorded information could impact one's willingness to use an EDRMS.

Six constructs were then selected from the literature to represent the three conceptual areas - performance expectancy, effort expectancy, social influence, perceived power security, and perceived value of records - which were all expected to drive the intention to use an EDRMS. The perceived 
value of records was developed for this research and is a new construct being introduced into the literature.

The conceptual research model is shown in Figure 3.1 on p. 58. The hypothesized path effects between the selected constructs were developed and discussed in Chapter 3 resulting in nine hypotheses.

\subsubsection{Research Design}

Having identified likely constructs from the literature and having formed a conceptual research model, the next step focused on the research design. The design consists of two main phases.

Phase I consisted of a qualitative methodological phase to develop a pool of potential measurement items relating to the conceptual research model and its constructs. This phase also addressed the first research objective, namely: To identify the factors that influence a user's intention to use an electronic recordkeeping system.

Phase II consisted of a quantitative methodological phase to validate the links between the measurement items and their constructs, and thereby test the research model. This phase addressed the second research objective, namely: To determine the relative importance of the factors that influence a user's intention to use an electronic recordkeeping system.

The final research design consisted of six stages. Each stage collected data and resulted in analysis that informed the next stage. Phase I (qualitative methods) comprised:

- Stage 1: Interviews - The interview stage consisted of a semi-structured interview (see Appendix A on p. 231) with 12 participants selected from the targeted research population. The interviews sought to gather data that could be used to validate the existing set of construct measurement items derived from the literature, explore potential measures for the new construct (perceived value of records), and search for additional items or factors that may impact a user's intention to use an EDRMS that were not specifically covered by the research model. See Section 5.2.4 (p. 86) to trace the development of the measurement items.

- Stage 2: Proofreading - The proofreading stage sought ten volunteers to check the proposed measurement item statements and verify that each statement was written clearly and unambiguously. Problematic measurement items were reworked to incorporate the best of the feedback 
and the final wordings were checked for tense and clarity. Only then were the measurement items tentatively associated with a parent construct from the research model. See Table 5.16 (p. 108) for a summary of the results and Appendix B (p. 235) for the annotated measurement items.

- Stage 3: Card Sorting - The card sorting stage focused on validating the proposed relationship between a measurement item and its parent construct. Card sorting (Moore \& Benbasat, 1991; Hinkle, 2008) was used to test both the convergent validity and discriminant validity of the measurement-item-to-construct relationship in order to inform the development of the final survey instrument and reduce the risk of poorly performing measurement items in the Phase II quantitative analysis.

Twenty-four participants were recruited from the research population to participate in the card sorting activity, with twelve focusing on closed card sorting (Spencer \& Warfel, 2004) and the remaining twelve focusing on open card sorting (Faiks \& Hyland, 2000).

The participants' qualitative sorting judgments were quantitatively analysed, resulting in a series of agreement frequency matrices - see Table 5.18 (p. 119) and Table 5.19 (p. 123) - and dendrograms - see Figure 5.2 (p. 118), Figure 5.3 (p. 124), Figure 5.4 (p. 125), and Figure 5.5 (p. 126).

The findings linked the measurement items to their parent constructs and supported the development of the survey instrument as part of the next stage.

Having developed a set of measurement items, the research then focused on a quantitative methodological approach. Phase II comprised:

- Stage 4: Survey Design - This stage focused on the selection of the survey delivery technology, the selection of the analysis approach (which dictated the form of the data to be collected), the creation of the survey instrument, and the development of the associated documentation and communication templates. See Appendix C (p. 239) for the final survey instrument and Appendix D (p. 249) for the associated documents and communication templates.

- Stage 5: Survey Pretest - This stage focused on bringing everything together for a "dress rehearsal" of the final survey. This stage sought to evaluate the distribution procedures, discover any production mistakes, 
learn whether people clearly understood all of the survey statements and questions, and provide an opportunity for feedback.

The pretest survey was sent to a sample of a dozen participants. The feedback was positive, and although some errors were noted and corrected, the feedback did not result in any major changes to the survey instrument.

- Stage 6: Survey - The final stage consisted of the survey data collection activity. The survey was sent to a panel of 254 employees of a large New Zealand public sector organization and resulted in 193 usable records. See Figure 7.1 (p. 140) for an analysis of the demographics of the participants and Figure 7.2 (p. 142) for a breakdown of their experience with EDRMS technologies. The raw survey data is presented in Appendix E (p. 257).

The research findings and model evaluation focused on the analysis of the quantitative data collected by the survey instrument.

\subsubsection{Research Findings and Model Evaluation}

The survey data were first examined using Principal Component Analysis (PCA) in order to determine how well each pool of measurement items reflected their target construct. The measurement items must be statistically unidimensional (i.e., they must share the same measurement dimensions) in order to adequately reflect their latent construct.

In order to select the best measures of each construct for use in the final structural equation model, each construct's pool of measurement items were compared in the PCA space. The Circle of Correlations was adopted as a common and practical method to visualize the data. See Section 7.3.2 starting on p. 145. Measurement items that did not cluster well were reviewed for the possibility of different semantic interpretations and other potential sources of error. Several measurement items were subsequently removed from the final measurement model.

The remaining measurement items formed the basis for the final analysis using Partial Least Squares Path Modeling (PLS-PM) (Vinzi et al., 2010; Chin, 1998b; Sanchez, 2013b; Tenenhaus et al., 2005). PLS-PM can be considered as two separate models. The outer (measurement model) consists of the relationship between the measurement items and their constructs. The inner (structural) model consists of the relationship between the constructs 
and thus represents the conceptual research model. The validity of the latter relies on effective measures of the former.

Having already been through the initial filter using PCA and the circle of correlations, the reduced set of measurement items comprising the outer model loaded well. The measures of fit (see Table 7.5 on p. 166 and Figure 7.11 on p. 168) were well above the required thresholds. In addition, all statistical measures of unidimensionality (see Table 7.6 on p. 169) were also well above the required levels. The outer model provided a strong statistical foundation for measuring the inner model and addressing the research question.

The next step was to evaluate the inner (structural) model. The PLS-PM algorithm calculated path coefficients for each of the hypothesized paths that make up the research model. These path coefficients provide an indication of the size of the effect that one construct has on another. These path effects were graphically plotted in Figure 7.12 on p. 173.

Once these were calculated, the statistical significance of the path coefficients was evaluated. See Table 7.10 (p. 176) for the inner model significance tests and Table 7.11 (p. 179) for the quality of the model fit. The bootstrapping results are presented in Appendix F on p. 265 and the final measured model is portrayed in Figure 7.14 on p. 181.

The survey data and measurements supported five of the nine hypothesized path effects. These are summarized on p. 182 and discussed in Section 8.3.1 starting on p. 203. The three most important constructs influencing use that emerged from this research were, in order of significance, the perceived value of records, effort expectancy, and social influence (see Figure 8.1 on p. 203).

\subsection{Contributions of the Research}

This research has made several contributions to the existing body of knowledge. These are divided into contributions which provide academic value and contributions which provide practitioner value.

\subsubsection{Academic Value of the Research}

The primary academic contribution of this research is the combination of existing IS technology acceptance theory with recordkeeping and its set of underlying theories. Information systems that are designed to support recordkeeping requirements are quite complicated, and they require the input of extensive recordkeeping metadata and an understanding of what records 
are and how records should be stored and organized. Furthermore, these systems expect end users to take on this responsibility.

This research sought to determine which factors influence a user's intention to use an electronic recordkeeping system. It tested the existing technology acceptance theory on a recordkeeping class of enterprise information system and found it wanting. Performance expectancy - the most important of the technology acceptance constructs - measured as insignificant in explaining the use of an electronic recordkeeping system.

Lee (2014) described TAM and UTAUT as being 'incomplete models' because they fail to take into account the social structure(s) in which the user operates. This research sought to test and expand on the traditional determinants of system use by including constructs representing the users' organizational context (which captures Lee's referenced social dimension) and knowledge interpretation (which focuses on the perceived value of records stored in the system), thereby combining aspects of structuration theory and the Records Continuum Model with technology acceptance theory.

The results suggest that TAM's perceived usefulness and/or UTAUT's performance expectancy may be too narrowly defined and culturally specific to work in all situations. The use of a performance lens for usefulness focuses on the competitive advantage afforded by the use of the technology as the primary driver of technology acceptance, and thus excludes other social drivers (including altruism) that occur when the primary beneficiaries of use are not solely the individual, but include the social structure(s) in which that individual operates.

This research also introduced a new construct, the perceived value of records, and provided a corresponding set of validated measurement items. This construct proved to have a high and significant path coefficient within the measurement model, and outperformed all other constructs.

During the search for new measurement items to reflect the perceived value of records, this research took the opportunity to recheck the nomological validity of the more established constructs and their associated measurement items. During this process, many of the traditional measurement items were further validated; however, this research also detected that the social influence construct was multidimensional. Structuration Theory was used to explain the three observed dimensions; furthermore, this multidimensionality could also explain why social influence has often been found to be insignificant in previous studies. This opens up opportunities for future research to explore this finding (see Directions for Future Research, Section 9.5, p. 227). 


\subsubsection{Practitioner Value of the Research}

As reliance on physical (paper) files reduces, an organization's documents and records are increasingly being stored only in an electronic (digital) format. Unfortunately, the success of organizational electronic recordkeeping systems in managing these records, as measured by acceptance and use, has been mixed.

The impact of low utilization of an organization's electronic recordkeeping system means that documents are not being properly managed, can be lost through destruction or deterioration, or else can be made irretrievable through lack of an indexing mechanism (Wilson, 1995). Loss of these records also has an impact on society. In the short term, this could lead to a loss of accountability, a cornerstone of our legal system and civilization; and in the long term, ultimately a loss of history (Johnston \& Bowen, 2005).

By identifying the factors that impact the use of electronic recordkeeping systems, this research will help software vendors (e.g., systems developers) by informing future strategies and prioritizing development work that target constructs likely to affect a user's intention to use the system. This research suggests that ease of use would have the greatest impact, whereas seeking to create gains in job performance would have little or no effect on use.

This research will also assist organizations (e.g., records managers and implementation project teams) by improving understanding of the motivations behind contribution behaviors. This knowledge will help practitioners to develop implementation and change management strategies that focus on addressing concerns likely to provide social barriers to use, as well as focusing on aspects of training, such as the importance of contextual metadata and an emphasis on the value of records, that will increase the perceived benefit of using systems.

\subsubsection{Implications of the Research}

In addition to the benefits for both researchers and practitioners, a number of observations can be made about the implications of this research.

Firstly, although it is expected that the measured path effects (and thus the interpretation of the model) will vary based on the local organizational context, this research has highlighted that the technology acceptance model has not worked as expected in a recordkeeping context. With the performance expectancy/perceived usefulness measurement effect being low and insignificant, this research has shown that the context of systems use is important. 
Recordkeeping systems in public sector organizations have a unique set of factors influencing use.

The large effect size of the new construct, perceived value of records, also has implications for the governance of organizations. This research has shown that those individuals placing a high value on records are more likely to be interested in managing those records in the provided EDRMS. Furthermore, Ashton and Reed (2011) argued that aligning the captured records with an organization's business processes is an excellent way to provide evidence of those business processes. Increased reliance on records, when combined with improved alignment of the records classification with business processes, could provide a way to automate the majority of the metadata capture (i.e, reduce effort), increase the transparency and accountability of the business processes, and provide trustworthy and strategically captured data with which to evaluate business performance. In other words, the EDRMS could be the next source for efficiency and effectiveness measures.

Finally, records bring both transparency and accountability, and depending on the organizational culture, these attributes can either be perceived as an asset (i.e, in a consensus organization) or as a liability (i.e., in a dissensus organization). Management should be aware of organizational culture when working on strategies to improve their recordkeeping policies and strategies (see Directions for Future Research, Section 9.5, p. 227).

\subsection{Limitations of the Research}

There were also certain limitations of this research that need to be recognized. They fall into three areas: limitations associated with the conceptual model, the research design, and the research instrument. These are discussed below.

\subsubsection{Limitations of the Conceptual Model}

The conceptual model was developed to represent three conceptual areas, including technology acceptance, organizational context, and knowledge interpretation. However, not all of the constructs selected to represent these three conceptual areas measured well.

Specifically, the constructs associated with organizational context presented measurement challenges. As suggested in the Directions for Future Research (Section 9.5, p. 227), the constructs representing the organiza- 
tional context conceptual area may be improved by focusing on the three structurational interactions of communication, power, and sanction.

\subsubsection{Limitations of the Research Design}

The primary limitations associated with the research design may be linked to constraints associated with the sample/sample frame and the selected level of analysis.

One source of concern may be the decision to sample a New Zealand public sector organization to represent the target population for the Stage 6: Survey data collection activity. To address this concern, it should be noted that the majority of the constructs and their measurement items have been sourced from studies from North America and that these have since been validated in additional studies internationally. By including those measurement items and retesting their nomological validity in this research (as assessed both in the qualitative phase's thematic analysis and in the quantitative phase's measures of statistical validity), we have assessed that those measures work similarly within a New Zealand context.

In addition, the literature in support of the challenges associated with EDRMS adoption comes from numerous locations around the globe, thus demonstrating that EDRMS acceptance challenges are not limited to New Zealand. Furthermore, the EDRMS system software used in the organization under study was a commercial off-the-shelf system developed overseas and imported into New Zealand. The challenges experienced in New Zealand are likely to be similar to those experienced by any organization using the same software.

Finally, New Zealand strives to stay current with global governance practices and recordkeeping policies, as evidenced by its regular adoption of, and contribution to, international standards. It is a global player. Given the quantitative measures of nomological validity and qualitative observations of the use of international standards, one can conclude that measurements made within New Zealand are highly likely to have applicability internationally and will likely reflect the realities associated with the adoption challenges of electronic recordkeeping systems wherever they may occur.

A second potential limitation of the research design was that it focused on a single organization and/or lacked group level variable(s) capable of reflecting and measuring possible differences between groups within an organization such as cross-level or multilevel conceptualizations of the research model's constructs (K. J. Klein \& Kozlowski, 2000). As such, the research design did 
not explore the organizational context constructs at the team or organization level nor did it explore the differences in the perceived value of records between groups within an organization. To do so would have required additional complexity such as selecting theoretically appropriate methods to conceptualize and measure multilevel constructs, justifying the levels of aggregation, and utilizing a different approach to analyzing the resulting multilevel models. As the conceptual research model was constructed on a foundation of validated constructs that were limited to single-level models, this research design also excluded analysis of potential aggregations. ${ }^{1}$ Nonetheless, future research could further explore the impacts of group behaviors on the use of electronic recordkeeping systems. See Section 9.5.4 on p. 229.

\subsubsection{Limitations of the Research Instrument}

It was not possible to know the strengths and weaknesses (and the statistical unidimensionality) of the measurement items without first measuring them. As such, many of the measurement items included in the research instrument were found not to cluster well during the PCA's circle of correlations. Several of the research instrument's items were subsequently discarded from the final PLS-PM outer measurement model.

By including more measurement items than were required to statistically reflect the constructs, this research instrument was necessarily a compromise: one which sought to measure the model and validate the measurement items at the same time (using the same data).

It is likely that several replications would be required to further refine and validate a final set of improved measurement items that could form a more succinct and parsimonious research instrument. Such an instrument would also likely include additional constructs or aggregation variables such as those suggested in the next section.

\footnotetext{
${ }^{1}$ Care must be taken to collect and analyze data at the same level as required to satisfy the research question. Attempting to deduce unit level interactions based on data collected at the aggregate level can be hindered by the ecological fallacy (Steel \& Holt, 1996). Similarly, attempting to infer group behaviors based on data collected at the unit level can be impacted by the atomistic fallacy (Diez, 2002).
} 


\subsection{Directions for Future Research}

There are many aspects of organizational recordkeeping systems that have not been explored. The primary direction of future research suggested by the findings and analysis consists of aligning various aspects of commonly used information systems constructs with Giddens' Structuration Theory. By aligning constructs and measurement items with this broad meta-theory, it will be possible to build new theory and linkages using a consistent foundation. This shared foundation could place information systems research within a contextual social framework that incorporates a robust view of the interactions and structures of society. Four major directions are suggested below.

\subsubsection{Improved Measures of Social Influence}

Social Influence was the third most important construct in the measurement model. As shared and networked information systems continue to expand and grow in importance, it is likely that social interactions concerning the use of these systems will similarly grow in importance.

As detected in Figure 7.5 (p. 152), the measures of the constructs that are claimed to be represented by social influence displayed additional clustering within the circle of correlations. The three clusters were imperfectly interpreted as aligning with Giddens' three social interactions of communication, power and sanction (Lewellen, Hooper, \& Oliver, 2014b).

The research model focused solely on social influence (representing the communication interaction) and on power. In this context, the sanctionbased measurement items were not suggested from the literature, nor did they emerge from the interviews. Nonetheless, approaching social influence using Giddens' three structurational interactions may provide improved theoretically-grounded measurement constructs that take into account the need for different types of social interactions to explain aspects of technology acceptance. A new set of social influence constructs would be ideal for use in future models, particularly those that require unidimensional measures and that take advantage of the additional sensitivity provided by modern SEM techniques. Potential research objectives include:

- Creation and definition of a new set of social influence constructs based on Giddens' three structurational interactions of communications, power, and sanction. 
- Development of a set of validated and unidimensional measurement items sufficient to measure these constructs and thereby integrate them into future structural models.

- Research that validates the appropriateness of the use of structurational interaction constructs in place of traditional social influence measures. For example, research that quantifies the latent social influence variable (in the principal component space) as measured in previous studies and determines the mathematical appropriateness of replacing the legacy construct(s) with the proposed structurational measures of social influence.

- Research into cross-level and multilevel conceptualizations of the proposed structurational measures of social influence in order to provide a richer understanding of the impact of team norms on individual behaviors and vice versa.

- Research into benchmarking the relative effect size of the different interactions (communication, power, and sanction behaviors) as they pertain to different technology acceptance and other IS use situations, particularly in organizations that exhibit different cultural attributes (e.g., on the consensus to dissensus continuum).

Furthermore, incorporating Giddens' structures and modalities into this future research agenda could provide additional insight into organizational context.

\subsubsection{Social Influence in Mandatory Use Situations}

The importance of social influence and its effect on technology acceptance is also likely to be influenced by the strength of policy associated with mandated use. In situations where the policy unambiguously requires use of a system, the reliance of social influence channels and the opinions of peers concerning the system are more likely to be ignored. However, when the policy concerning use is either ambiguous or not well publicized, it would follow that users would rely more heavily on cues from their peers and managers.

This avenue of future research could aim at determining the relative importance of communication and sanction social interactions within mandatory use situations taking into account cultural context and mode of transmission of these policies. For example, it is expected that a campaign to present clear 
organizational policies and expectations would effectively reduce the need and reliance on communication interactions, while increasing the potential role and importance of sanction interactions as a means to boost compliance with the stated policies.

\subsubsection{Aligment with the Records Continuum Model}

Another avenue of future research could focus on the development of constructs that better represent the evidentiality dimension of the records continuum. On p. 201, the revised definition of the perceived value of records included both a content dimension and a context dimension. Only the content dimension was measured in this research; however, as illustrated in Table 8.2 (p. 202), the addition of measures on the perceived value of recordkeeping context could provide additional insight into how users (and organizations) value their information as well as explaining or predicting the technological choices they make in managing that information.

For example, if future EDRMS implementations continue to rely on end users to contribute documents to the system, it may be necessary to promote awareness of the purpose of contextual metadata so that they can willingly participate as good eCitizens within a culture that values records.

\subsubsection{Development of a Multilevel Theoretical Model}

This research consisted of a single-level research design; however, future research could seek to develop a multilevel conceptualization of the research model that may be capable of providing a richer understanding of the determinants of EDRMS use.

For example, a cross-level moderator model analysis may be capable of showing that variables at different levels of analysis (e.g., a group-level variable and an individual-level variable) could interact to predict a measurable outcome at the lower unit level of analysis (K. J. Klein \& Kozlowski, 2000, p. 219). A future multilevel study may demonstrate that the proposed structurational social influence constructs are both multilevel and homologous within an organizational context. In other words, the explanatory capability of the conceptual research model may be supported across different levels of the organizational system, thereby providing additional validation of the constructs and the functional relations linking the constructs. A similar approach could be used to further explore the proposed content and context 
aspects of the perceived value of records across functional groups within an organization and/or groups between organizations.

\subsubsection{Benchmarking the Prevalence of the Knowledge Management Discourses}

This research also cited Schultze and Stabell (2004)'s four discourses of knowledge management research (see Figure 2.16 on p. 52). Furthermore, it has detected the prevalence of their neo-functionalist discourse, which identifies knowledge as an asset, worthy of sharing and storing for the future.

This research has not attempted to quantify or benchmark the distribution of people among the four proposed discourses. To address this gap, future research into identifying the contextual distribution of people among the knowledge discourses could provide additional insight into situations where electronic recordkeeping systems adoption has not gone as planned. This applies particularly if the distribution varies between different cultures and/or contexts.

\subsection{Chapter Summary}

This concluding chapter begins with a brief overview and summary of the research process; first discussing the research gap, the research objectives, and the development of the conceptual research model. The focus then moves to summarizing the research design and the subsequent findings and model evaluation.

The chapter then focused on the contributions of the research, highlighting both the academic value and the value and implications to practitioners who may be struggling to improve the acceptance and use of their EDRMS investment.

The limitations of the research were then discussed. These were categorized into the limitations of the conceptual model, the limitations of the research design, and the limitations of the research instrument. Finally, several directions for future research were both identified and discussed.

In seeking to discover the factors that influence a user's intention to use an electronic recordkeeping system, this research not only uncovered and measured those factors, but also provided several paths for future research into the social use of technology. This chapter is finished, but the research continues... 


\section{Appendix A}

\section{Interview Instrument}

The following semi-structured interview instrument was used during Stage 1: Interviews of the qualitative research phase from April-June 2012.

The indicative questions cover the general areas suggested by the conceptual research model as well as provide open-ended questions to seek out other potential factors. The first group of questions places the individual within the organization and determines their approximate level of EDRMS experience. Important questions are in bold.

[Demographics] - to be used for reporting the interview sample

- What is your current role/position?

- What was your previous occupation?

- observable age, gender

[Initial Situation]

- Would you consider yourself a 'regular' users of the EDRMS? Describe what kinds of things do you store in the EDRMS (general categories).

- Do you generally use the EDRMS for filing things you receive, or for things you create?

- Do you feel you should be storing more? Less?

The second section will explore the applicability of existing measurements. The intention is not to verify old instruments, but rather explore aspects of them that would be applicable to my model.

[Social Influence/Subjective Norm]

- Thinking of your co-workers (peers), have they expressed any opinions about the EDRMS? 
- Thinking of your manager, have they expressed any opinions about the EDRMS?

- How important is their (e.g., co-workers, managers) opinion to you? Do you feel that your overall opinion of the system is in alignment with theirs?

- Is the use of the EDRMS included in any of your performance review criteria? Do you think it should be? Explain.

[Perceived Ease of Use]

- Have you had training in the use of the EDRMS? Was it sufficient?

- Thinking of some normal activities you perform in the EDRMS, please describe the effectiveness of its interface? Is it logical and easy-to-use? Do you understand why it is set up the way it is? Explain.

- Have you ever had any difficulties or frustrations with the system? Describe how these have affected your opinion of the system?

- In terms of other large systems you have used, provide some adjectives you would use to describe the EDRMS.

[Perceived Usefulness]

- Do you believe that using the EDRMS improves your job performance? How?

- Do you believe that having a shared electronic recordkeeping system improves your personal efficiency? The organization's efficiency? Explain.

- Why do you think that the organization has invested so much time and effort in creating and maintaining the EDRMS?

- Do you know where other people store their documents in the system?

- Let's say that you need a piece of information (e.g., a document) created by your team. Thinking of all of the places that information could be stored - on a peer's desk, in a physical file, on email, in a shared drive, in the EDRMS, etc.--where would you first go looking for information? Would you expect to find it in the EDRMS?

- Let's say you need a piece of information created by another team or business unit. Again, thinking of all the places that information might be stored, what would be the process or approach you would follow when looking for that information?

- Do you believe that digital information is stored 'safely' in your organization? Do you believe that physical documents are stored 'safely'? Do you think that digital information, as it is currently managed in your business unit, would be easily accessible in 10 years time? 
[Perceived Power Security]

- Would it be a 'good thing' if everyone stored everything (e.g., emails, documents, notes, etc.) in the EDRMS? Explain.

- Assuming that someone diligently stored every document and every pertinent email in the EDRMS, how would these actions affect their 'influence' in the work place?

- Would you feel more or less job security in knowing that all of the records of your past work, contacts, and decisions had been recorded in an EDRMS? Explain.

- Do you ever have any concerns that people may look at the content you have loaded into the system and think negatively of you or your work? Do you think that management looks through your content submissions? Do you think they should? Why?

- Do you ever look at records created by your co-workers? Do you feel that this is expected and normal, or a form of invasion of privacy?

The third section will explore a new construct and gain insight on potential measurement factors:

[Perceived Value of Records]

- In your own words, describe what you think a 'record' is. Describe some of its characteristics.

- Why do you think that Parliament is interested in legislating recordkeeping - e.g., the Public Records Act? Is the value of the records worth the effort in capturing them? Are your records worthy of capture?

- Do you rely on your business unit's filing system (note: it doesn't have to be in the EDRMS)? Is the unit's filing system reliable? What criteria would you use to judge its reliability?

- Can you access information that is 10 years old easily? Can you access current up-to-date information easily? How much do you rely on organizational information as part of your job?

- To what extent do you document the processes leading up to a decision? Would you find it useful/comforting to know that decisions in the organization were backed up by documentation, emails, etc.? Would you expect others to use those records to influence/guide their decisionmaking? Would you?

- How do you relate 'records' and 'accountability' in your own words? Does your opinion match that of others in the organization? Do you ever create records as "insurance" to protect yourself or your reputation? Do you ever avoid creating records for the same reason? How do you value accountability as compared to efficiency or effectiveness? 
- How do you feel (emotionally) when you permanently lodge information attributed to you into a recordkeeping system? Explain.

- Do you 'trust' the factual nature of your organization's physical records? Would you trust them more (or less) if they were all held in an EDRMS?

- Do you have any other thoughts concerning the general 'value' of records?

The final section will draw everything together and explore their intention to contribute information to electronic recordkeeping systems:

[Intention to Use]

- Will you continue to use the EDRMS in the future?

- As more people use the system, do you expect that you will be storing more of your content in the EDRMS (e.g., emails, notes, documents, etc.)?

- Do you contribute information immediately upon creation? Or do you let it pile up and then do some recordkeeping when you have time? How does your contribution style compare to your peers?

- What categories of information do you generally NOT contribute into an EDRMS. Why?

[Conclusion]

- Please list some good or helpful things that an EDRMS would provide to you personally.

- Are there any other points you'd like to raise that haven't already been covered?

Thank the participant!! 


\section{Appendix B}

\section{Proofreading of Stage 1 Items}

In Section 5.2 .4 (p. 86), the initial pool of Stage 1 measurement items was formed as a combination of items taken from (or adapted from) the literature and measurement items that were created by the author to represent emergent themes from the interview stage.

As many of the measurement items were new and untested, and those taken from the literature were of different tone and tense, it was important that the wording of the measurement items be aligned and tested for meaning, comprehension, and grammar. To this end, Stage 2: Proofreading recruited participants to do just that. See Section 5.3 (p. 107).

A 3-page printed feedback instrument containing the Stage 1 measurement item statements was provided to each of the proofreading participants, and their combined feedback was transcribed by the author onto a single form in order to better understand the statements that are problematic and to seek patterns in the feedback. This combined master form records feedback for each statement and tally marks for instances of identical or similar feedback. In addition, those who thought the statement was fine were tracked using tally marks in the left margin.

The scanned combined (master) feedback instrument is reproduced over the next three pages. 


\section{Master (Combined)}

INSTRUCTIONS: Please review the following statements. I am particularly interested in any that you feel are vague, unclear, or difficult to understand.

- Please place a "tick" next to ones that are OK.

- Please suggest changes to ones that are problematic-e.g., adding clarifications, changing the wording, etc. The goal is to create clear and unambiguous statements.

And thanks for your help!

Matt

[ Pefine "system" and state in HLL CAPS

L Align Throughout items

What kind?

PE01 I would find the system useful in my job.

$$
\text { what kind? }
$$

HKII PE02 Using the system enables me to accomplish tasks more quickly.

HTIII PE03 Using the system increases my productivity.

|HWI PE04 If I use the system, I will increase my chances of getting [recognition in the workplace]

$$
\text { What ? yntron? }
$$

HW PE05 Using [Search] allows me to locate[important] records effectively.

$$
\text { I will be able to find/retrieve it) II }
$$

XII III PE06 When I store a record in the system, I am confident it will be there in the future.

$$
\text { I trust that... (capability of System v. my use) unit? org? }
$$

HX III PE07 The system can effectively manage the security required for[our] sensitive records.

WHII PE08 I find the document classification in the system [to be] intuitive.]

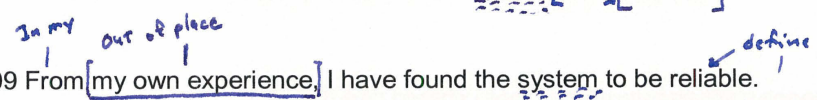

HII PE09 From [my own experience,] I have found the system to be reliable. I

$$
\text { align w/ cest of SYSTEM ?: }
$$

HIIII PE10 I know when I am supposed to use the [electronic recordkeeping] system in my job.

$$
\text { who? } 1 \text { it? org? }
$$

WHIII PE11[We] the telectronic recordkeeping]system as a way to achieve one or more of our business goals. what kind? Unsure...

IXIII PE12 The integration of the [electronic recordkeeping]ssystem with my other office programmes and tools saves me time.

$$
\begin{aligned}
& \text { Uitimately saving Suggest: "The SYSTEM integrates) } \\
& \text { S Saves me time. }
\end{aligned}
$$

$1 \Rightarrow$ "The SYSTEM integrates with my other office programmes and 1 tool ultimately saving me time." 


\section{OK}

UHF IfI PE13 I have noticed that the system is often slow in its operation.

? Reports Docs!

UX IIII PE14 I rely on[reports]generated from the system as]one of the tools to manage and achieve my job responsibilities. I...isystem to Report on the Status/timeliness, as...

WH EE01 My interaction with the system[would be]clear and understandable. $\leftarrow$ confusing II is? was? Il of"I find it easy..."

HWII EE02 It [would be] easy forme to become skillful at using the system.

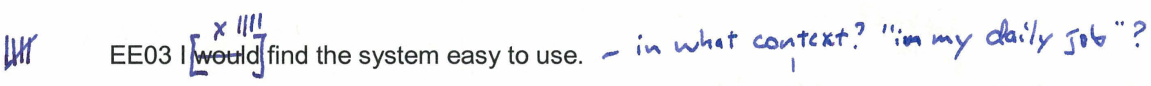

HW III EE04 Learning to operate the system is easy for me.

WII III EE05 I use the system often enough to maintain my skill [with] its use.

III assume the same thing?

If III EE06 The process of filing]-or[contributing a record]- is easy and straightforward.

WH EE07 I have no trouble searching for records in the system.

HIII EE08 I can learn how to do administrative tasks'vithout difficulty.

HII III EE09 I can access the recordkeeping system from most of ms Office? if not, Then programs.

H| |||| $\mid$ EE10 The system is flexible enough to support my needs as well as the organisation's requirements.

HII II SI1 People who influence my behaviour think that I should use the system.

HIIU SI2 People who are important to me think that I should use the system.

HI SI3 The senior[management] of this[business] has been helpful in the use of the system.

HW HI In general, the organization has supported the use of the system. 


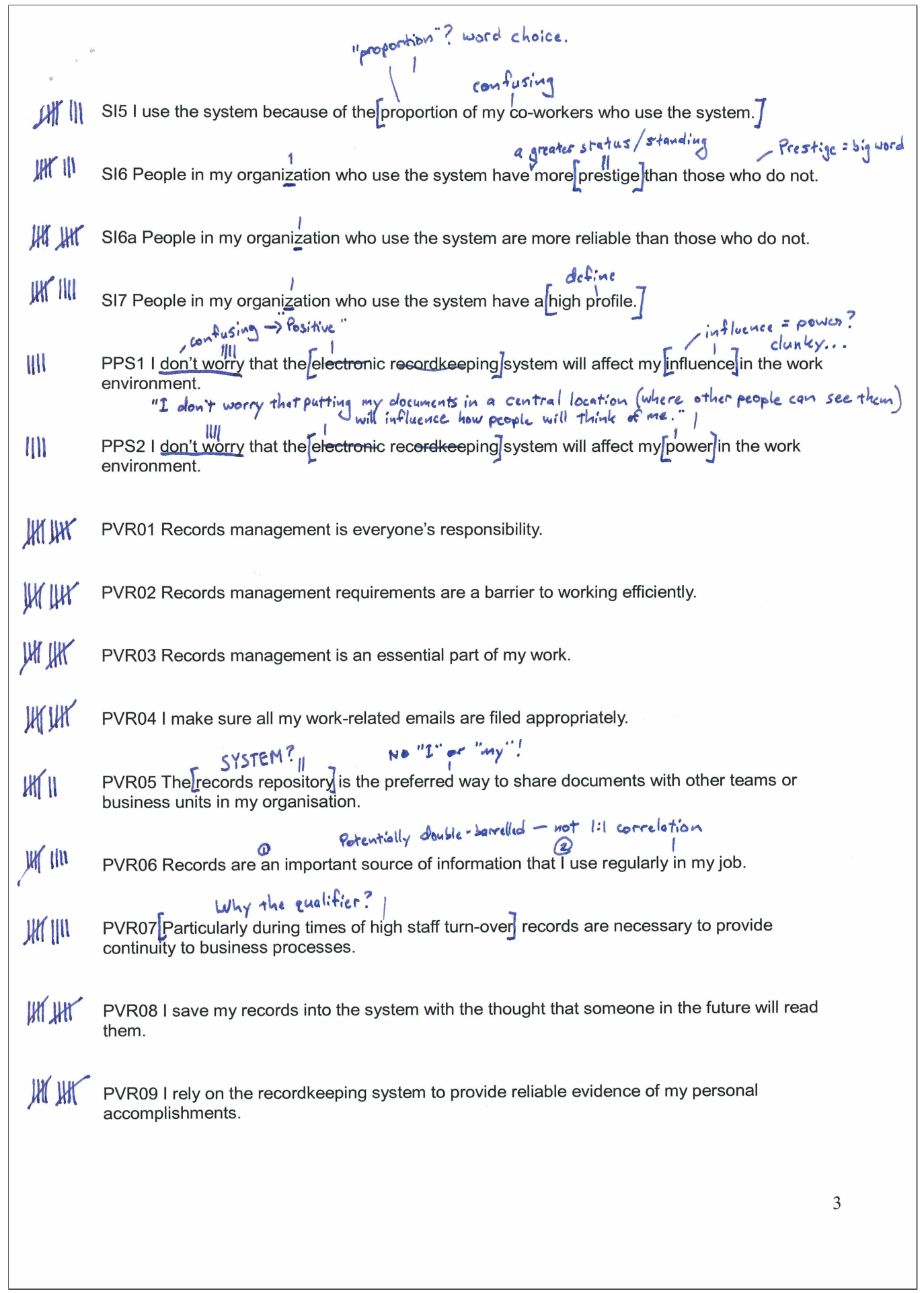




\section{Appendix C}

\section{Final Survey Instrument}

The Survey Instrument was created using Qualtrics (http://qualtrics.com/). The Qualtrics Research Suite is a popular on-line product that is designed specifically for market research, including the development of on-line surveys. Qualtrics was licensed to the researcher under the Victoria University of Wellington's site license.

The final survey instrument consisted of 7 pages (or 'screens') of questions, followed by an automated page that thanks the participant and confirms the capture of their answers.

Screenshots of the complete survey instrument as they were seen by participants are provided on the next several pages. 


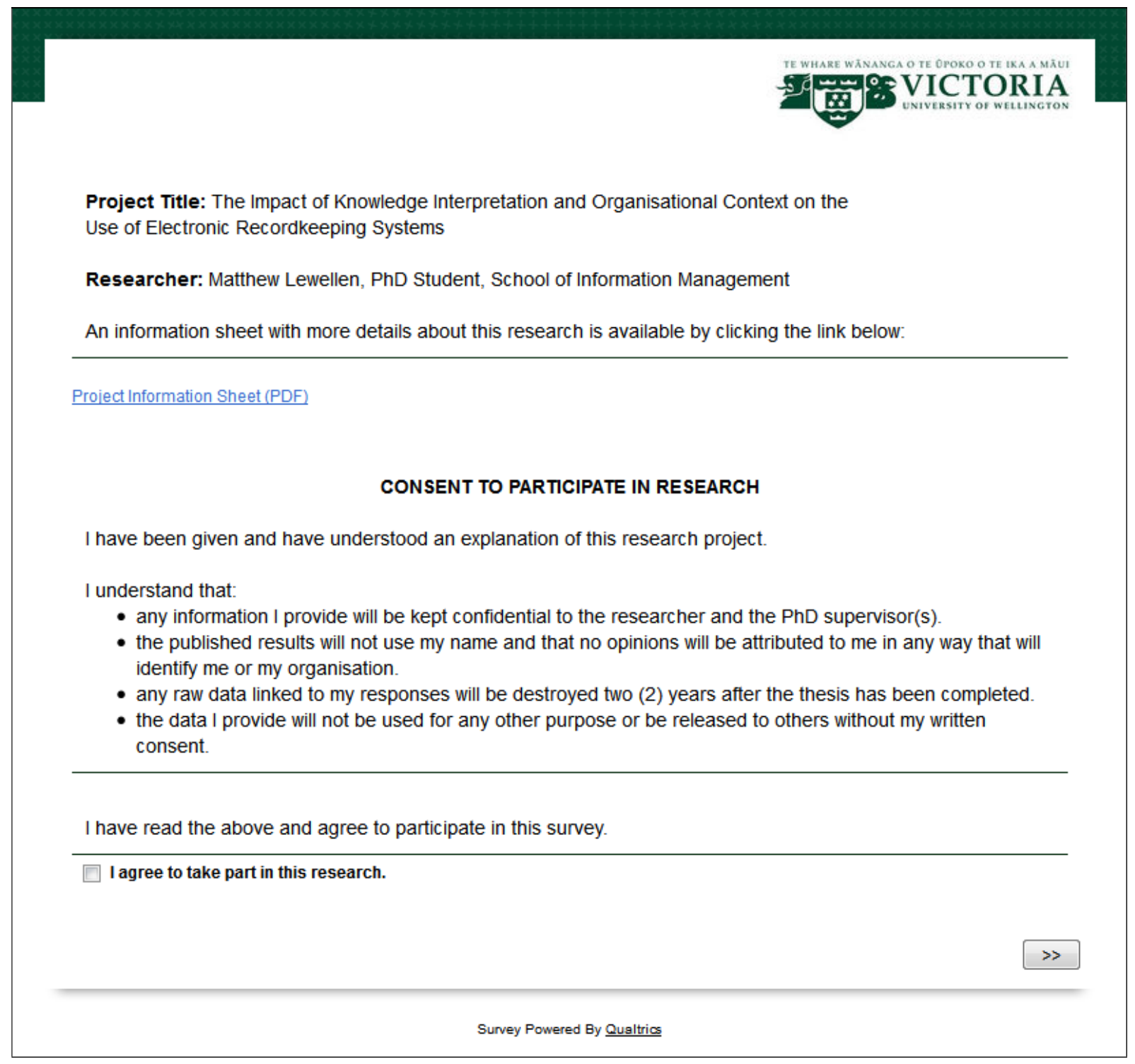

Figure C.1: Survey Instrument (Page 1) Consent to Participate 


\begin{tabular}{|c|c|c|c|c|c|}
\hline \multicolumn{6}{|c|}{$\begin{array}{l}\text { This survey focuses on the use of your organisation's electronic recordkeeping system. In your case, the focus is } \\
\text { on or other shared drives is not of interest to this study. } \\
\text { With regard to the ease-of-use of your organisation's electronic recordkeeping system, please state the extent } \\
\text { to which you agree or disagree with the following statements. }\end{array}$} \\
\hline & $\begin{array}{l}\text { Strongly } \\
\text { Disagree }\end{array}$ & Disagree & $\begin{array}{c}\text { Neither } \\
\text { Agree nor } \\
\text { Disagree }\end{array}$ & Agree & $\begin{array}{l}\text { Strongly } \\
\text { Agree }\end{array}$ \\
\hline $\begin{array}{l}\text { I find the system's interface (e.g., its menus and layout) to } \\
\text { be clear and understandable. }\end{array}$ & ○ & ○ & ○ & ○ & ○ \\
\hline I find it easy to become skillful at using the system. & ○ & 0 & ○ & 0 & ○ \\
\hline I find the system easy to use in my job. & O & O & O & O & O \\
\hline Learning to operate the system is easy. & 0 & ○ & ○ & 0 & ○ \\
\hline $\begin{array}{l}\text { I use the system often enough to maintain my skill in its } \\
\text { use. }\end{array}$ & ○ & ○ & ○ & ○ & ○ \\
\hline $\begin{array}{l}\text { The process of contributing a record to the system } \\
\text { (electronic filing) is easy and straightforward. }\end{array}$ & ○ & $\odot$ & ○ & $\bigcirc$ & O \\
\hline I have no trouble searching for records in the system. & O & O & ○ & O & ○ \\
\hline $\begin{array}{l}\text { I can learn how to do administrative tasks in the system } \\
\text { without difficulty. }\end{array}$ & ○ & ○ & ○ & ○ & ○ \\
\hline $\begin{array}{l}\text { I can access the system directly from other computer } \\
\text { applications--e.g., directly from Microsoft Office. }\end{array}$ & ○ & ○ & ○ & O & ○ \\
\hline I find that the records are logically organised in the system. & ○ & 0 & 0 & ○ & ○ \\
\hline & & & & & $\gg$ \\
\hline
\end{tabular}

Figure C.2: Survey Instrument (Page 2) Effort Expectancy construct 


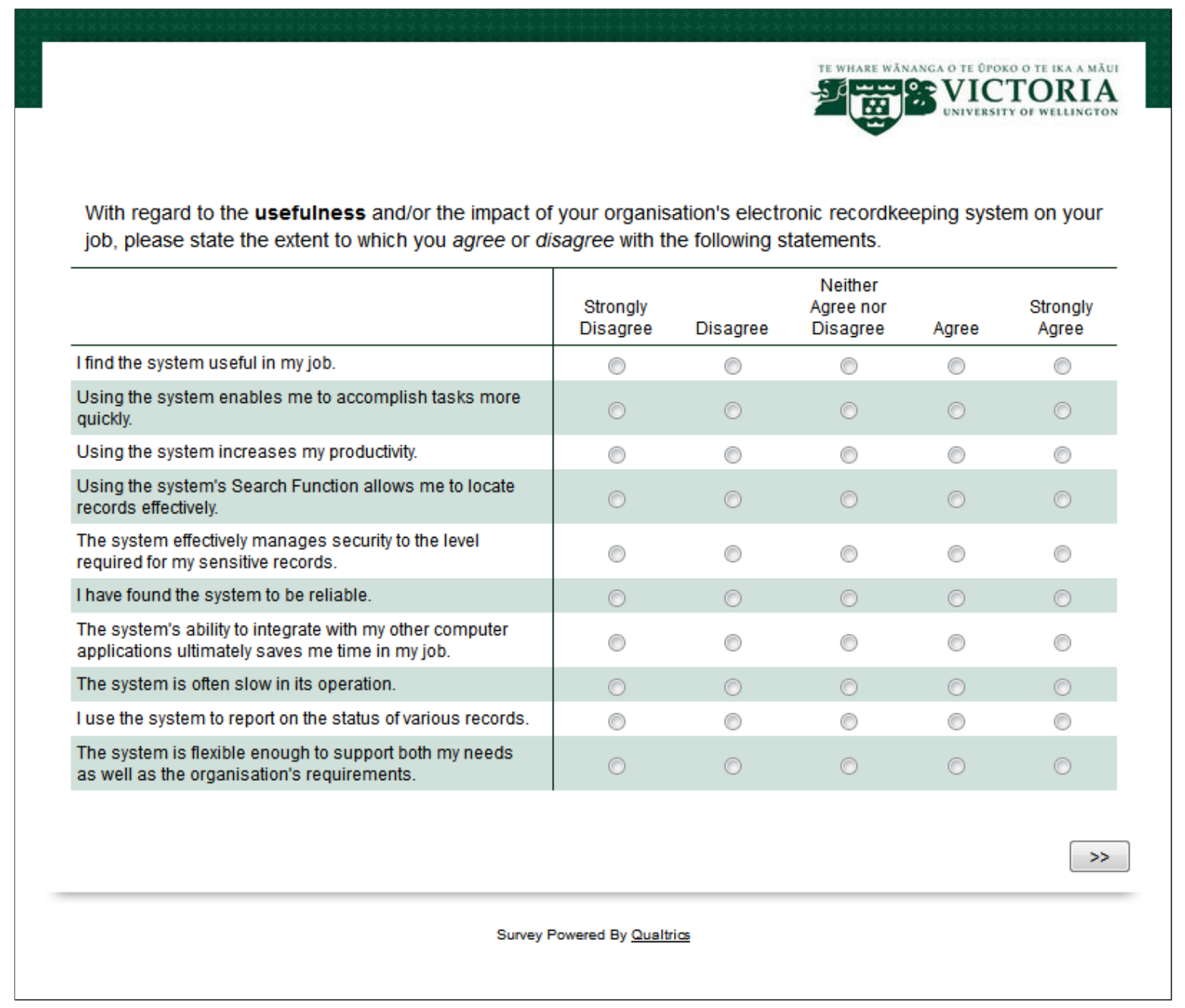

Figure C.3: Survey Instrument: Page 3-Performance Expectancy construct 


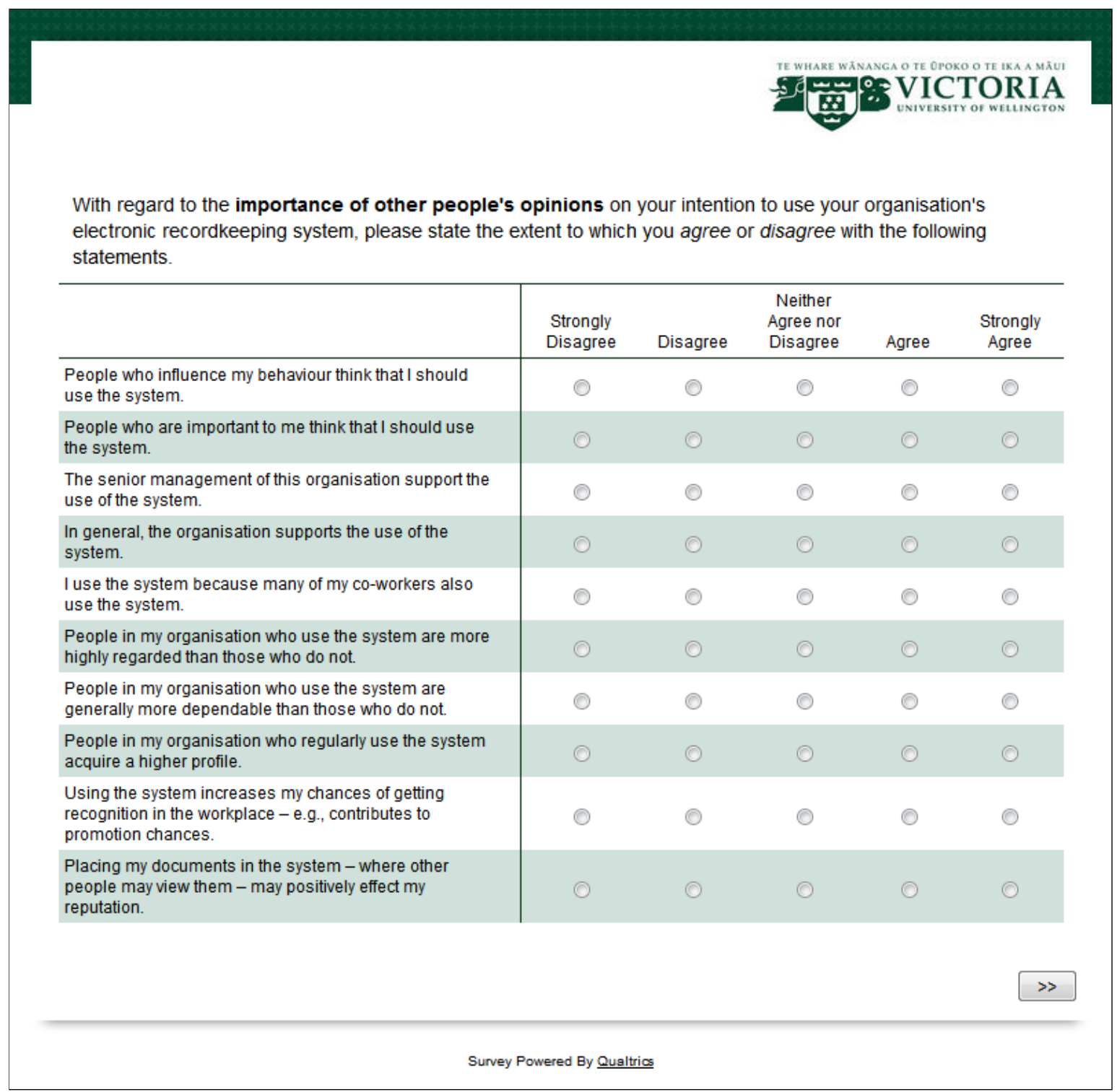

Figure C.4: Survey Instrument (Page 4) Social Influence construct 


\begin{tabular}{|c|c|c|c|c|c|}
\hline \multicolumn{6}{|c|}{$\begin{array}{l}\text { The use of a shared repository for all of your records may effect aspects of your power or influence at work. } \\
\text { Please state the extent to which you agree or disagree with the following statements. }\end{array}$} \\
\hline & $\begin{array}{c}\text { Strongly } \\
\text { Disagree }\end{array}$ & Disagree & $\begin{array}{l}\text { Neither } \\
\text { Agree nor } \\
\text { Disagree }\end{array}$ & Agree & $\begin{array}{c}\text { Strongly } \\
\text { Agree }\end{array}$ \\
\hline $\begin{array}{l}\text { By recording my knowledge in the system, I will be less } \\
\text { valuable to the organisation as a source of knowledge. }\end{array}$ & ○ & O & ○ & ○ & O \\
\hline Use of the system decreases my power over others. & ○ & O & ○ & 0 & ○ \\
\hline $\begin{array}{l}\text { By placing my records in the system, I feel that I have more } \\
\text { control over them. }\end{array}$ & ○ & ○ & ○ & ○ & ○ \\
\hline $\begin{array}{l}\text { By putting my documents into the system, there is a } \\
\text { greater potential for people to judge my work. }\end{array}$ & ○ & ○ & ○ & ○ & ○ \\
\hline $\begin{array}{l}\text { Use of the system increases my influence over other } \\
\text { people. }\end{array}$ & ○ & ○ & O & ○ & ○ \\
\hline \multicolumn{6}{|c|}{$\begin{array}{l}\text { When considering the importance of Records and Recordkeeping, please state the extent to which you } \\
\text { agree or disagree with the following statements. }\end{array}$} \\
\hline & $\begin{array}{c}\text { Strongly } \\
\text { Disagree }\end{array}$ & Disagree & $\begin{array}{l}\text { Neither } \\
\text { Agree nor } \\
\text { Disagree }\end{array}$ & Agree & $\begin{array}{c}\text { Strongly } \\
\text { Agree }\end{array}$ \\
\hline Records management is everyone's responsibility. & O & $\odot$ & ○ & O & ○ \\
\hline $\begin{array}{l}\text { Records management requirements are a barrier to } \\
\text { working efficiently. }\end{array}$ & ○ & ○ & $\bigcirc$ & ○ & ○ \\
\hline Records management is an essential part of my work. & ○ & O & ○ & ○ & ○ \\
\hline $\begin{array}{l}\text { I make sure all my work-related emails are filed } \\
\text { appropriately. }\end{array}$ & ○ & ○ & ○ & ○ & ○ \\
\hline $\begin{array}{l}\text { I rely on records to remind me of details of my earlier } \\
\text { work. }\end{array}$ & ○ & O & $\odot$ & ○ & ○ \\
\hline $\begin{array}{l}\text { I regularly refer to records to obtain information needed in } \\
\text { my job. }\end{array}$ & ○ & 0 & ○ & ○ & ○ \\
\hline $\begin{array}{l}\text { Particularly during times of high staff-turnover, records are } \\
\text { necessary to provide continuity to my organisation's } \\
\text { business processes. }\end{array}$ & O & O & O & ○ & ○ \\
\hline $\begin{array}{l}\text { I save my records into the system with the thought that } \\
\text { someone in the future will read them. }\end{array}$ & 0 & ○ & ○ & ○ & O \\
\hline $\begin{array}{l}\text { I have used records to provide reliable evidence of my } \\
\text { personal accomplishments. }\end{array}$ & O & ○ & 0 & ○ & ○ \\
\hline $\begin{array}{l}\text { My organisation relies on records as a way to achieve one } \\
\text { or more of its goals. }\end{array}$ & ○ & O & ○ & ○ & ○ \\
\hline Well managed records improve accountability. & ○ & ○ & ○ & ○ & ○ \\
\hline & & & & & \\
\hline
\end{tabular}

Figure C.5: Survey Instrument (Page 5) Perceived Power Security and Perceived Value of Records constructs 


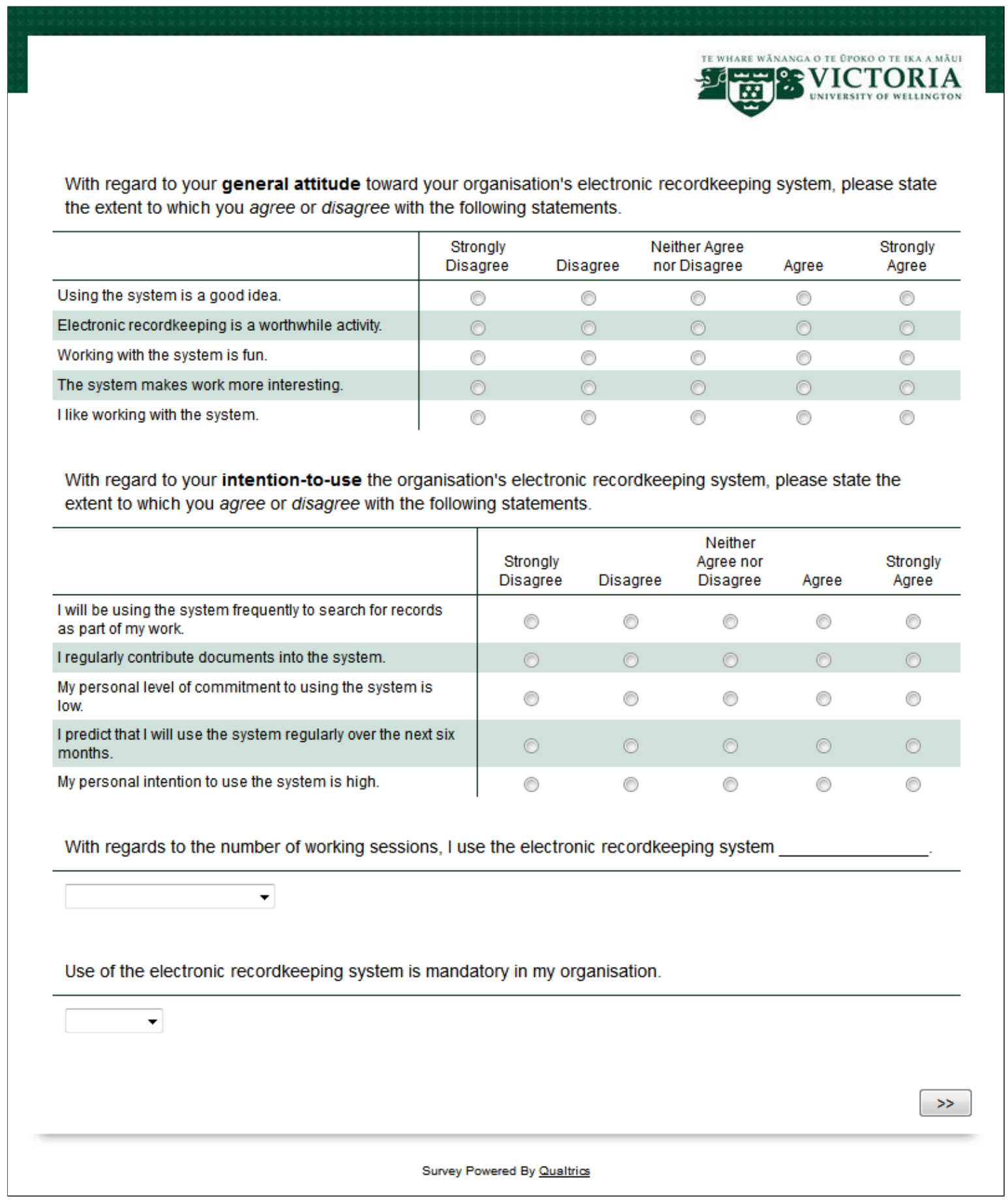

Figure C.6: Survey Instrument (Page 6) Attitude and Intention-to-use constructs 


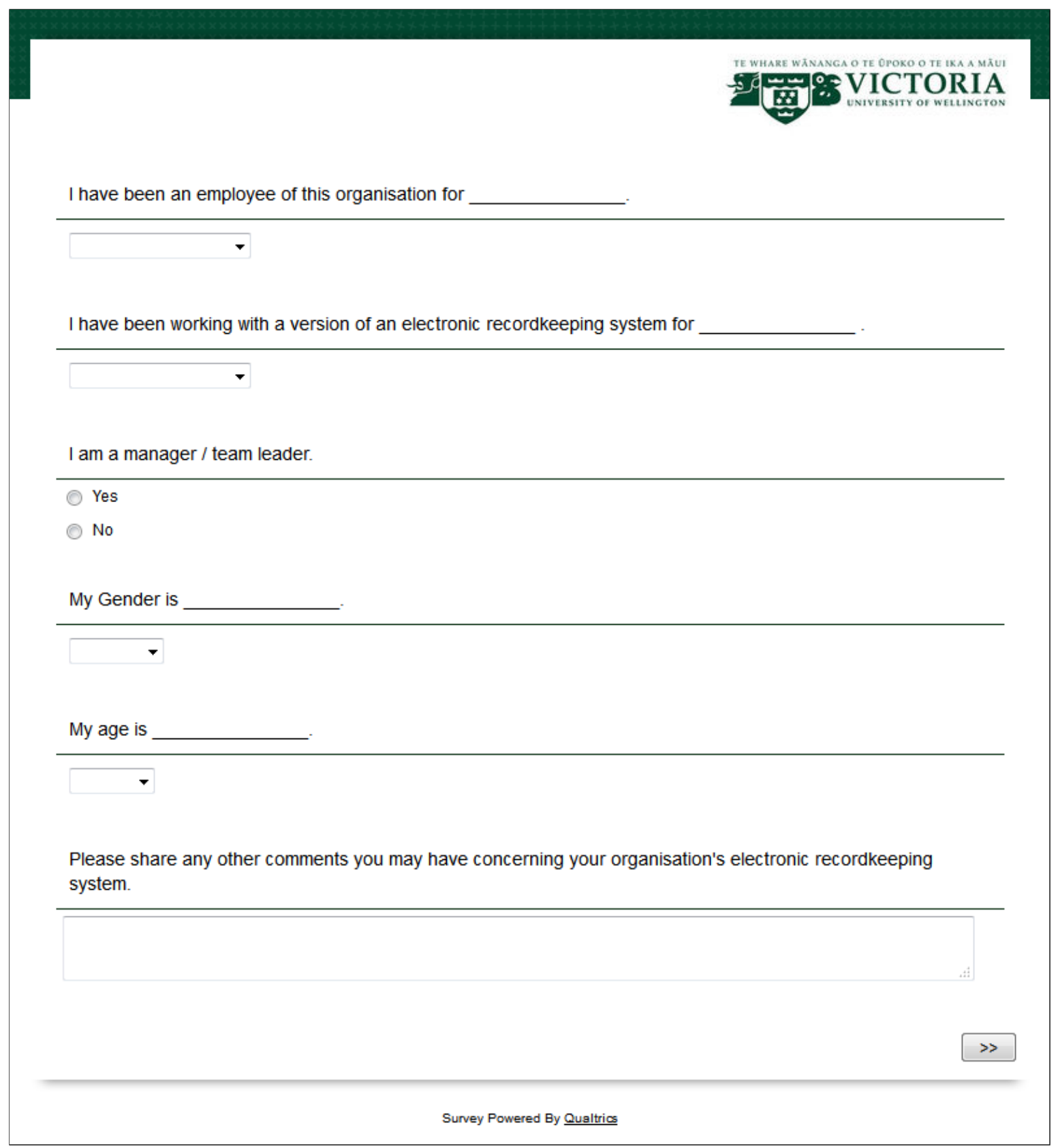

Figure C.7: Survey Instrument (Page 7) Demographics 


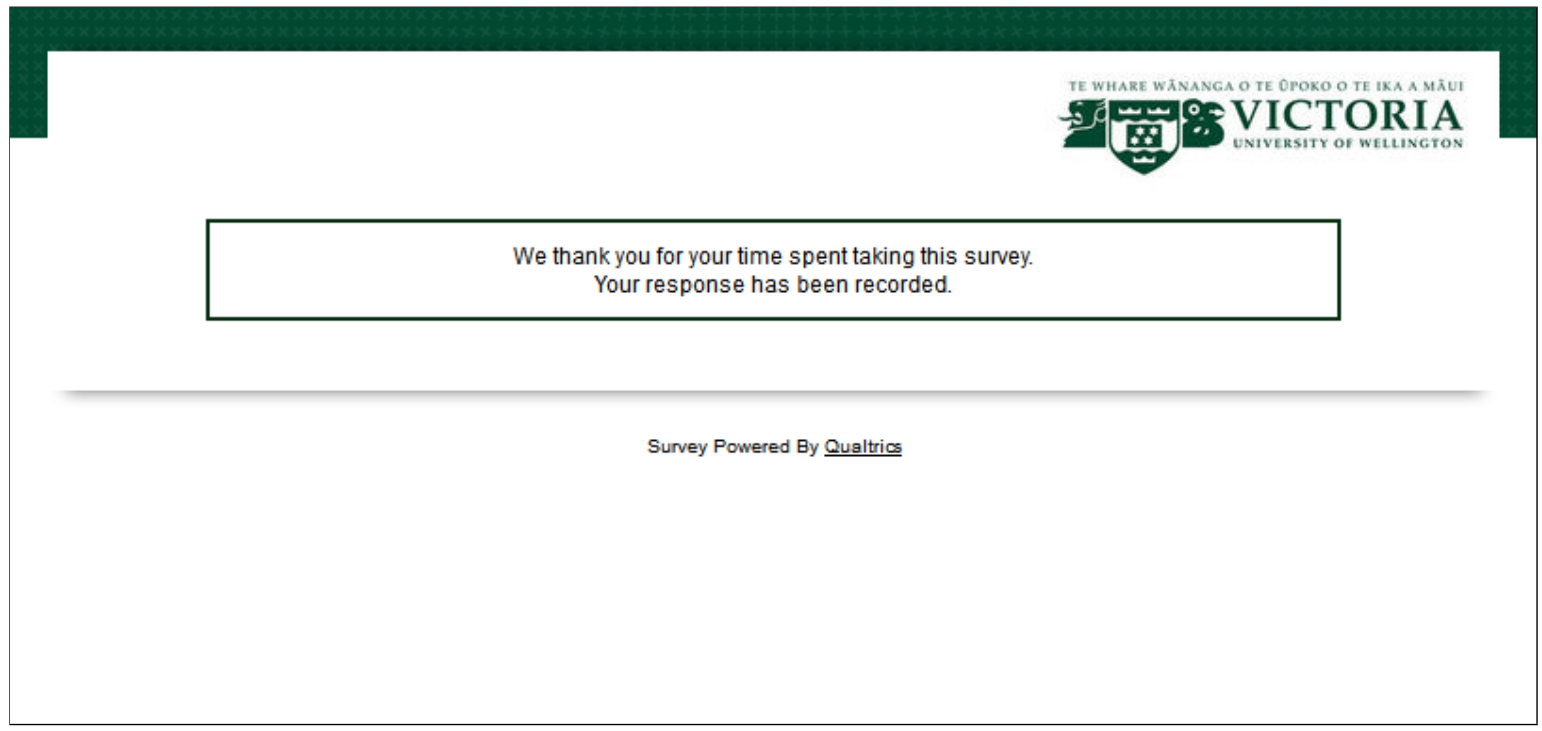

Figure C.8: Survey Instrument (Page 8) Thank you and confirmation of completion

\section{Demographic Scales}

The scales for many of the demographic questions were available as drop-down boxes within the survey instrument. Many of these were common frequency or time divisions and were available as a 'default' Qualtrics scale selection. The selected 'selection scales' are provided below for the sake of completion:

\section{Demographic Questions}

\section{Selection Scale}

With regard to the number of working sessions, Never

I use the electronic recordkeeping system:

Less than once a month

Once a month

2-3 Times a month

Once a week

2-3 Times a week

Daily

Use of the electronic recordkeeping system is mandatory in my organization

Yes

No

Not sure 
Demographic Questions-continued Selection Scale

I have been an employee of this organization for:

6 to 12 months

1 to 2 years

2 to 5 years

5 to 10 years

More than 10 years

I have been working with a version of an

Less than 6 months electronic recordkeeping system for:

6 to 12 months

1 to 2 years

2 to 5 years

More than 5 years

My Gender is:

male

female

My age is:

18-24

$25-34$

$35-44$

$45-54$

$55+$

*** End Section *** 


\section{Appendix D}

\section{Survey Communications Templates}

In an effort to minimize non-response (and thereby minimize non-response bias), Sivo et al. (2006) recommend two strategies: 1) selecting the smallest random sample from the target population that will provide sufficient power and accurately determining the effect size, and 2) "using Dillman's empirically supported Tailored Design Method (TDM) to minimize non-response" (Sivo et al., 2006, p. 355).

The Tailored Design Method was initially put forward in 1978 and, given its continued success, it continues to be the primary distribution method for reducing survey non-response (Dillman, 2009; Sivo et al., 2006). The method is grounded in social exchange theory, and includes a number of necessary elements, including: 1) a user-friendly questionnaire, 2) a multi-contact strategy, and 3) personalized correspondence. ${ }^{1}$

Dillman stressed the importance of tone and voice, and recommended that each contact in the multi-contact strategy use a different tone of voice and/or method of delivery. In an effort to reduce the perceived barriers to participation, he also recommends including the questionnaire with each contact (Dillman, 2009).

With this in mind, a series of email templates were built in Qualtrics, based roughly on the recommended paragraph order and structure (Dillman, 2007). A link to the Research Information Sheet and a link to the online survey was included with every communication (except for the 'thank you'

\footnotetext{
${ }^{1}$ Originally designed for mailed (paper) questionnaires, the original elements also included a return envelope with real first class stamps and a token prepaid financial incentive (Sivo et al., 2006)
} 
notes), and each email was personalized in an effort to build trust with the participants. In addition, the emails were scheduled to go out at 11:00am, a time of day when people might be ready for a change of activity.

The contact strategy included five contacts:

1. Introductory email

2. Reminder email \#1

3. Reminder email \#2

4. Reminder email \#3

5. Thank you email

The email templates were created in Qualtrics (http://qualtrics.com/), which can automatically track who has responded and who has not responded in order to optimally target the correct people with each mailing. In addition, the emails could be scheduled ahead to be sent at specific times, supporting a "fire and forget" approach to the contact strategy. The email templates for each stage - as well as details about their timings - are included below. Note that the email templates have been adjusted to hide the identity of the organization. 


\section{Introductory Email Template}

On 06 November 2013 at 11:00am, the following email template was sent to a panel of 254 people. It includes Qualtrics mail-merge field codes that customized each email and personalized it to the panel member. 'XXXXX' has been used to hide the identify of the organization.

[From] matthew.lewellen@vuw.ac.nz

[Subject] Electronic Recordkeeping Survey

Dear $\$\{\mathrm{~m}: / /$ FirstName $\}$,

I am a PhD student at Victoria University of Wellington as well as a past employee of XXXXX involved with the XXXXX electronic recordkeeping system.

As part of my degree, I am undertaking a research project to investigate the factors that employees consider when deciding whether to contribute documents into their organisation's electronic recordkeeping system - in your case: XXXXX. A better understanding of how people view and interact with these recordkeeping systems could influence how they are set-up and how people are trained, which could also improve legislative compliance and the quality and value of this organisational asset.

EDRMS RESEARCH - Information Sheet.pdf [LINK - see Figure D.1]

I have sought and obtained formal approval for you to participate in this online survey. The survey is short: it will only take between 7-10 minutes to complete.

Please complete the survey by close-of-business on Friday, 15 November 2013. Your opinion matters!

Follow this link to the Survey: $\$\{1: / /$ SurveyLink?d=Take the Survey $\}$

Or copy and paste the URL below into your internet browser: $\$\{1$ ://SurveyURL $\}$

Thank you for your participation.

Regards,

Matthew

Matthew Lewellen

PhD Student

Victoria University of Wellington

Follow the link to opt out of future emails:

$\$ 1: / / O p t O u t L i n k ? d=C l i c k$ here to unsubscribe 


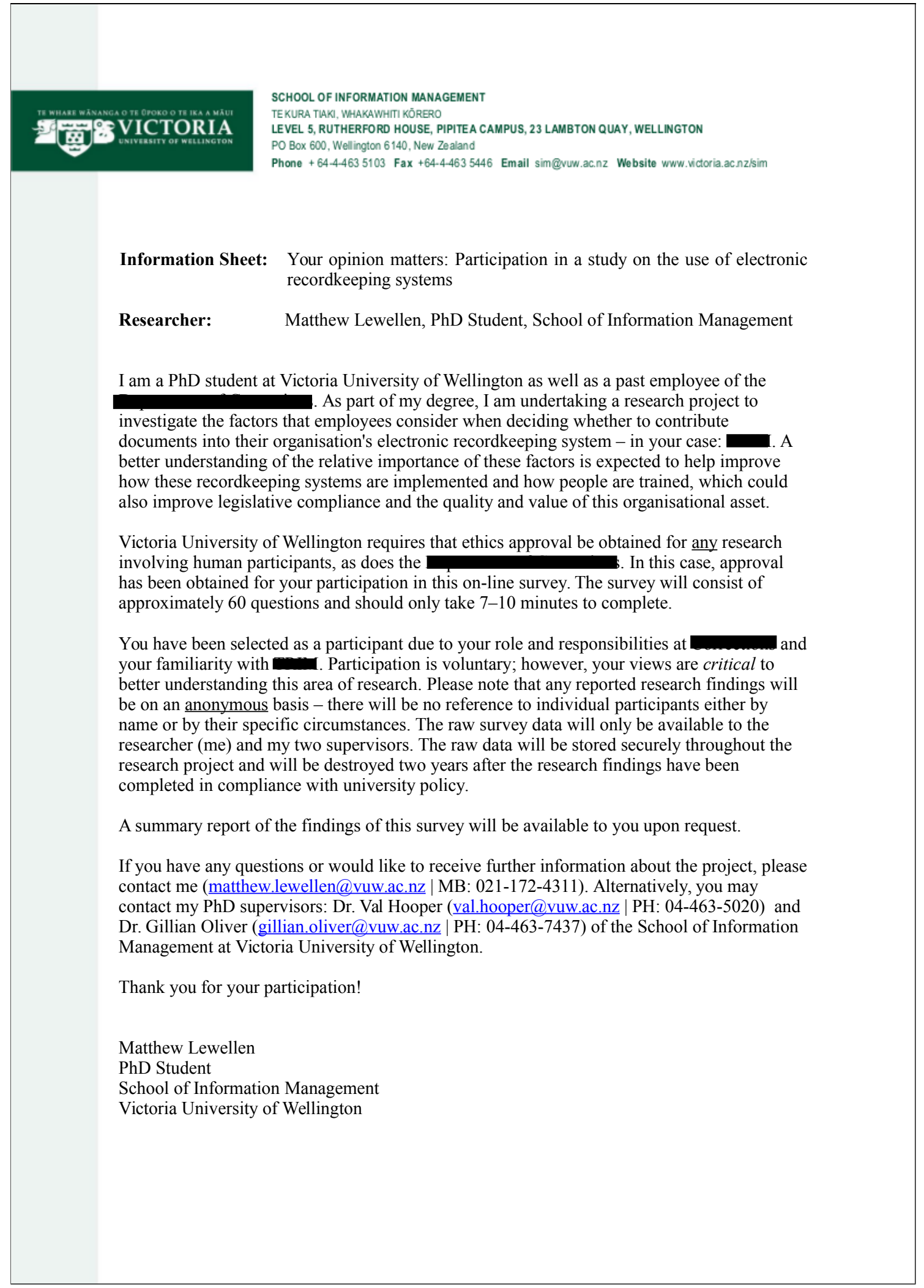

Figure D.1: Research Information Sheet - provided to participants as a link on all communications as well as on the online survey consent page. 


\section{Reminder Email Template \#1}

On 12 November 2013 at 11:00am, an email template was sent to a panel of 131 people. This was the first reminder email as per the schedule recommended by the modified Tailored Design Method (Dillman, 2009).

[From] matthew.lewellen@vuw.ac.nz

[Subject] Electronic Recordkeeping Survey (reminder)

Dear $\$\{\mathrm{~m}: / /$ FirstName $\}$,

This is a gentle reminder that the Electronic Recordkeeping Survey is still open this week, and I have yet to hear from you. The survey is short: it will only take between 7-10 minutes to complete.

Several people have asked whether the survey is only targeted at people who use XXXXX regularly. Since the purpose of the study is to look at the factors that contribute to use, I am as interested in hearing from 'infrequent users' as I am in hearing from 'regular users.' All opinions matter!

Follow this link to the Survey: $\$\{1: / /$ SurveyLink?d=Take the Survey $\}$

Or copy and paste the URL below into your internet browser:

$\$\{\mathrm{l}: / /$ SurveyURL $\}$

Also, if you started the survey, but did not yet complete it, the attached link will take you back to where you left off.

In case you missed last Wednesday's original survey invite, I have also reattached the information sheet that provides more information about this research project:

EDRMS RESEARCH - Information Sheet.pdf [LINK - see Figure D.1]

Please complete the survey by close-of-business on Friday, 15 November 2013.

Thank you for your participation.

Regards,

Matthew

Matthew Lewellen

PhD Student

Victoria University of Wellington

Follow the link to opt out of future emails:

$\$ 1: / / O p t O u t L i n k ? d=$ Click here to unsubscribe 


\section{Reminder Email Template \#2}

On 15 November 2013 at 11:00am, this extension email template was sent the remaining 80 people who had not yet completed the survey. The time extension was designed as an opportunity to encourage higher participation amongst the remaining panel members.

[From] matthew.lewellen@vuw.ac.nz

[Subject] Electronic Recordkeeping Survey - extension

Dear $\$\{\mathrm{~m}: / /$ FirstName $\}$,

The Electronic Recordkeeping Survey was due to have been completed today; however, I have received a number of emails from people requesting additional time to complete the survey due to heavy work load, planned leave, etc. A high response rate is important for the survey to have statistical validity. As such, the survey will remain open one more week, and I encourage you to take advantage of this to share your opinions. The survey is short: it will only take between 7-10 minutes to complete.

Follow this link to the Survey: $\$\{1: / /$ SurveyLink?d=Take the Survey $\}$

Or copy and paste the URL below into your internet browser:

$\$\{1: / /$ SurveyURL $\}$

Remember that I am equally interested in the opinions of both 'frequent' and 'infrequent' XXXX users. Also, if you started the survey, but did not yet complete it, the attached link will take you back to where you left off.

In case you missed the original survey invite, I have attached the information sheet that provides more information about this research project:

EDRMS RESEARCH - Information Sheet.pdf [LINK - see Figure D.1]

Please complete the survey by close-of-business on Friday, 22 November 2013.

Thank you for your participation.

Regards,

Matthew

Matthew Lewellen

PhD Student

Victoria University of Wellington

Follow the link to opt out of future emails:

$\$ 1$ ://OptOutLink?d=Click here to unsubscribe 


\section{Reminder Email Template \#3}

On 21 November 2013 at 11:00am, this reminder email template was sent the remaining 41 people who had not yet completed the survey.

[From] matthew.lewellen@vuw.ac.nz

[Subject] Electronic Recordkeeping Survey (final reminder)

Dear $\$\{\mathrm{~m}: / /$ FirstName $\}$,

You still have time to participate in the Electronic Recordkeeping Survey (which closes tomorrow)!

I encourage you to share your opinions about XXXXX and be part of this research project. The survey is short: it will only take between 7-10 minutes to complete.

Follow this link to the Survey:

$\$\{1: / /$ SurveyLink?d=Take the Survey $\}$

Or copy and paste the URL below into your internet browser:

$\$\{1: / /$ SurveyURL $\}$

Remember that I am equally interested in the opinions of both 'frequent' and 'infrequent' XXXXX users. Also, if you started the survey, but did not yet complete it, the attached link will take you back to where you left off.

Please complete the survey by close-of-business on Friday, 22 November 2013.

Thank you for your participation!

Regards,

Matthew

Matthew Lewellen

PhD Student

Victoria University of Wellington

Follow the link to opt out of future emails:

$\$ 1: / / O p t O u t L i n k ? d=$ Click here to unsubscribe 


\section{'Thank You' Email Template}

On 22 November 2013 at 06:00pm, this Thank You email template was sent to all people who had completed the survey.

[From] matthew.lewellen@vuw.ac.nz

[Subject] Electronic Recordkeeping Survey - Thank You

Dear $\$\{\mathrm{~m}: / /$ FirstName $\}$,

I wanted to thank you personally for taking the time to complete my electronic recordkeeping survey.

The survey has enjoyed a very respectable $85 \%$ response rate. This high response rate bodes well for a solid analysis and some compelling conclusions.

Thank you again!

Regards,

Matthew

Matthew Lewellen

PhD Student

Victoria University of Wellington 


\section{Appendix E}

\section{Final Survey Data}

The survey responses were recorded in Qualtrics using a 1-5 (Disagree-Agree) Likert Scale. The survey design (see Appendix C) aligned the measurement item questions by construct, resulting in multiple question groups. The groups of interest included:

- Effort Expectancy (Ease of use) - see Table E.1 (p. 258)

- Performance Expectancy (Usefulness) - see Table E.2 (p. 259)

- Social Influence - see Table E.3 (p. 260)

- Perceived Power Security - see Table E.4 (p. 261)

- Perceived Value of Records - see Table E.5 (p. 262)

- Intention to Use - see Table E.6 (p. 263)

A summary of the responses to each measurement item (in the form of bar charts using the original Likert Scale responses by construct) are provided on the following pages. 


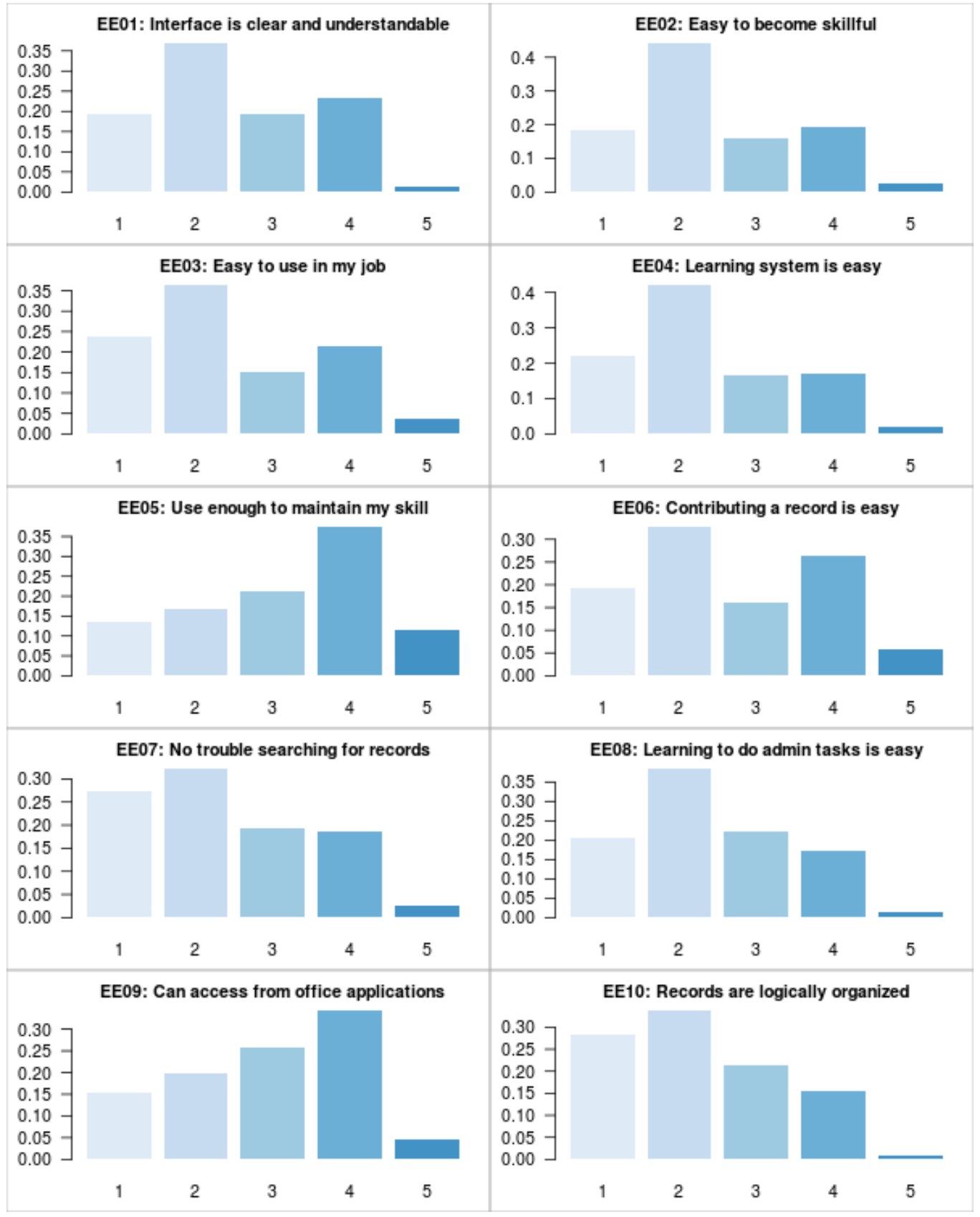

Figure E.1: Bar chart of survey responses: effort expectancy (ease of use) measurement items 


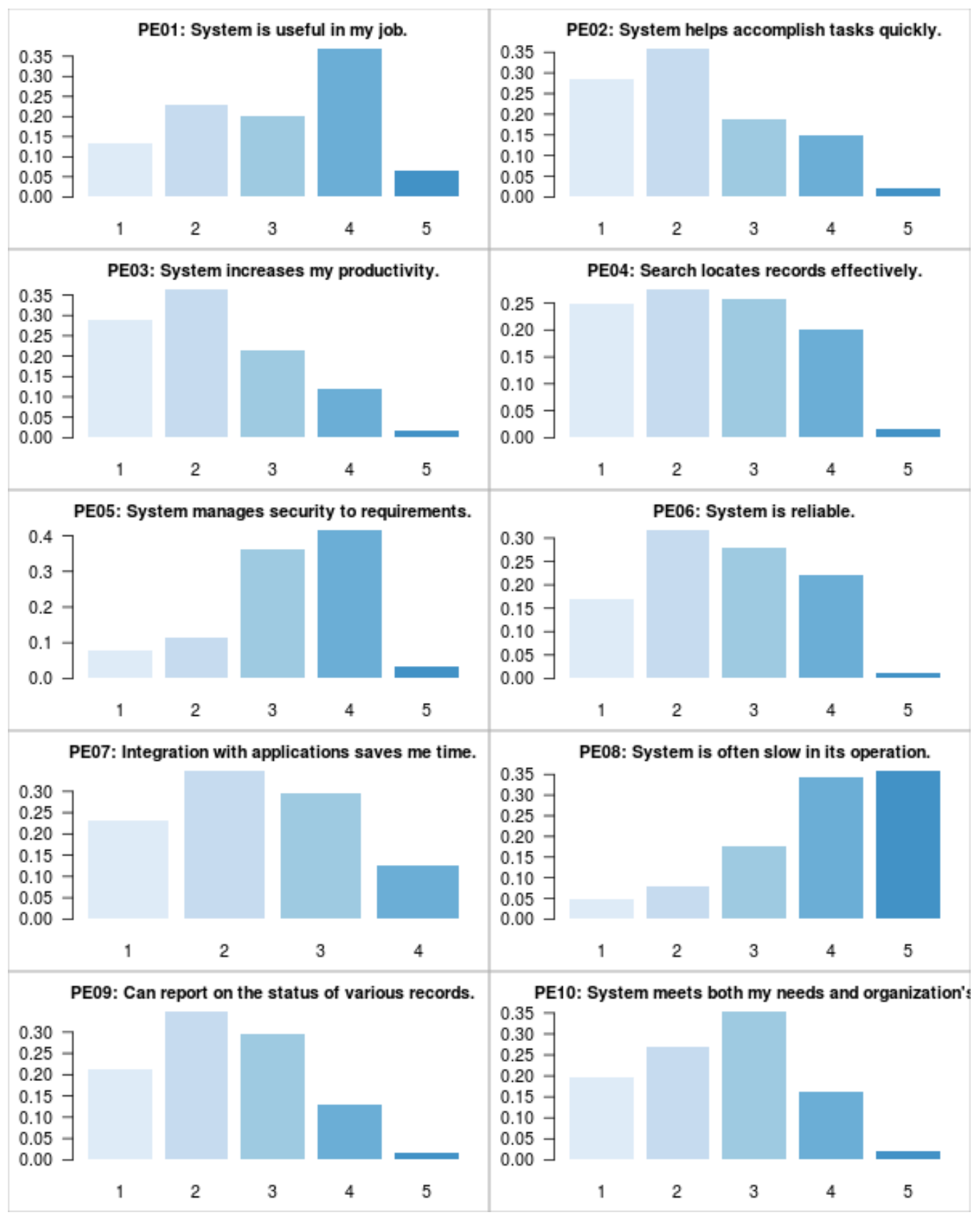

Figure E.2: Bar chart of survey responses: performance expectancy (usefulness) measurement items 


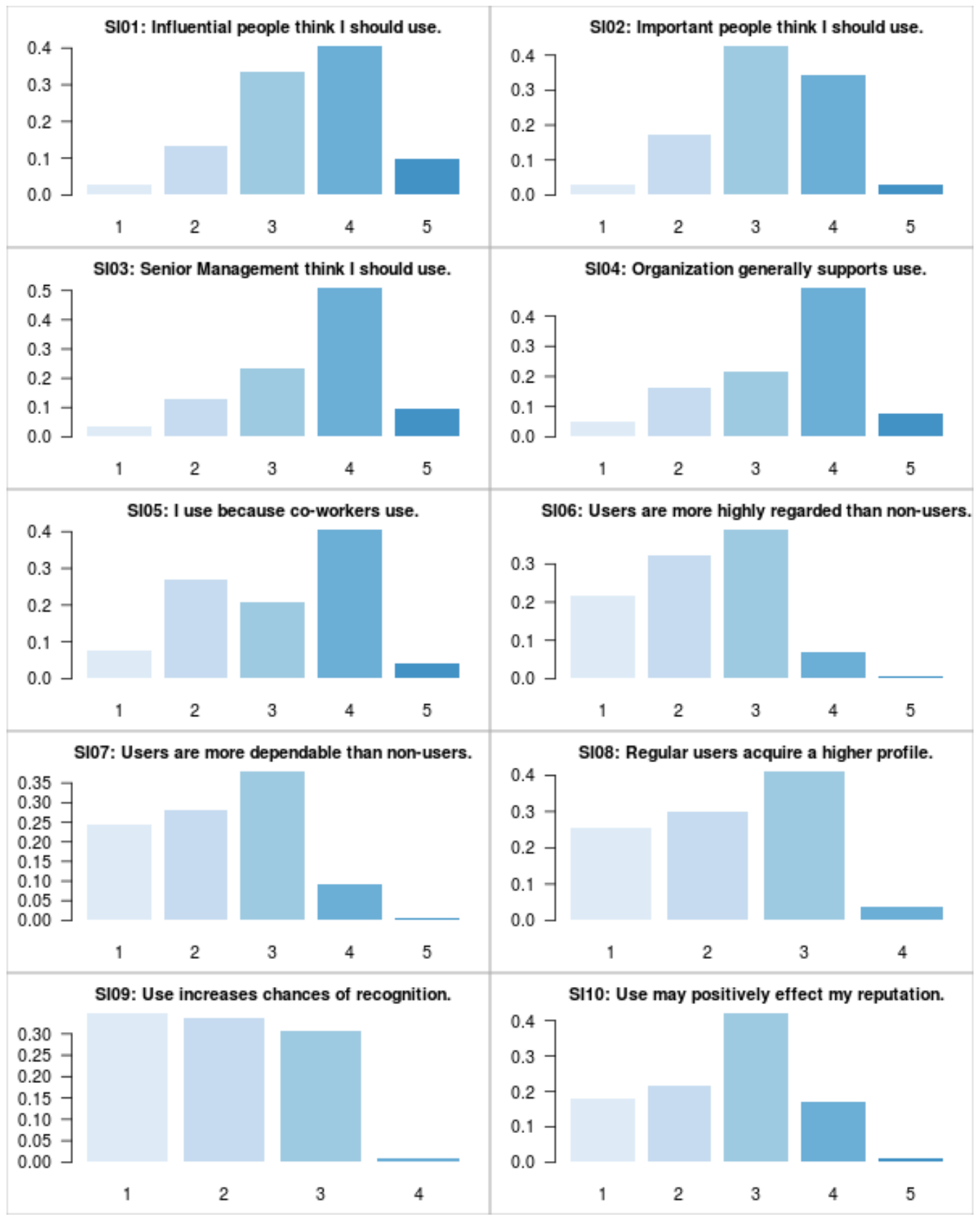

Figure E.3: Bar chart of survey responses: social influence measurement items 


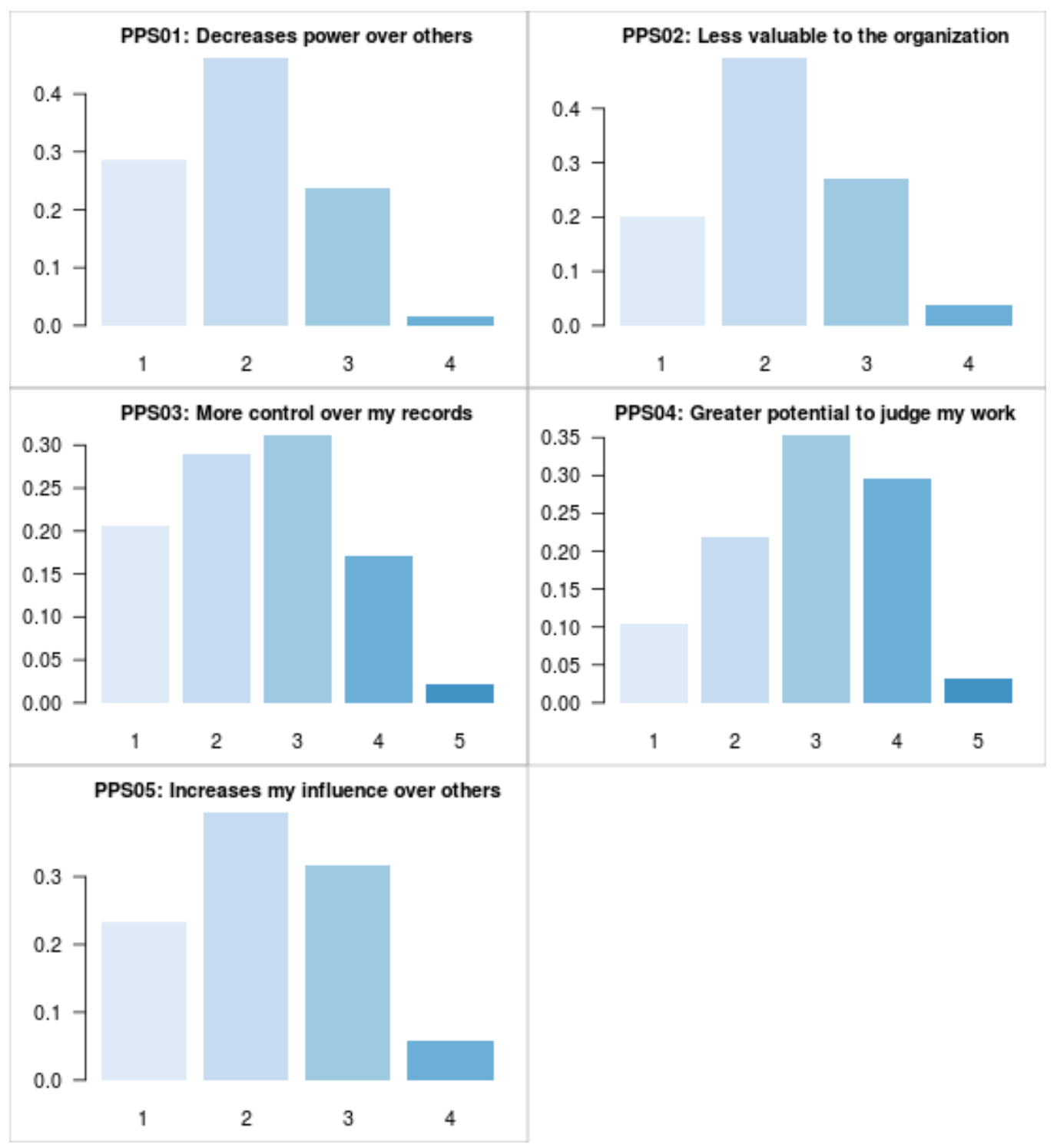

Figure E.4: Bar chart of survey responses: perceived power security measurement items 


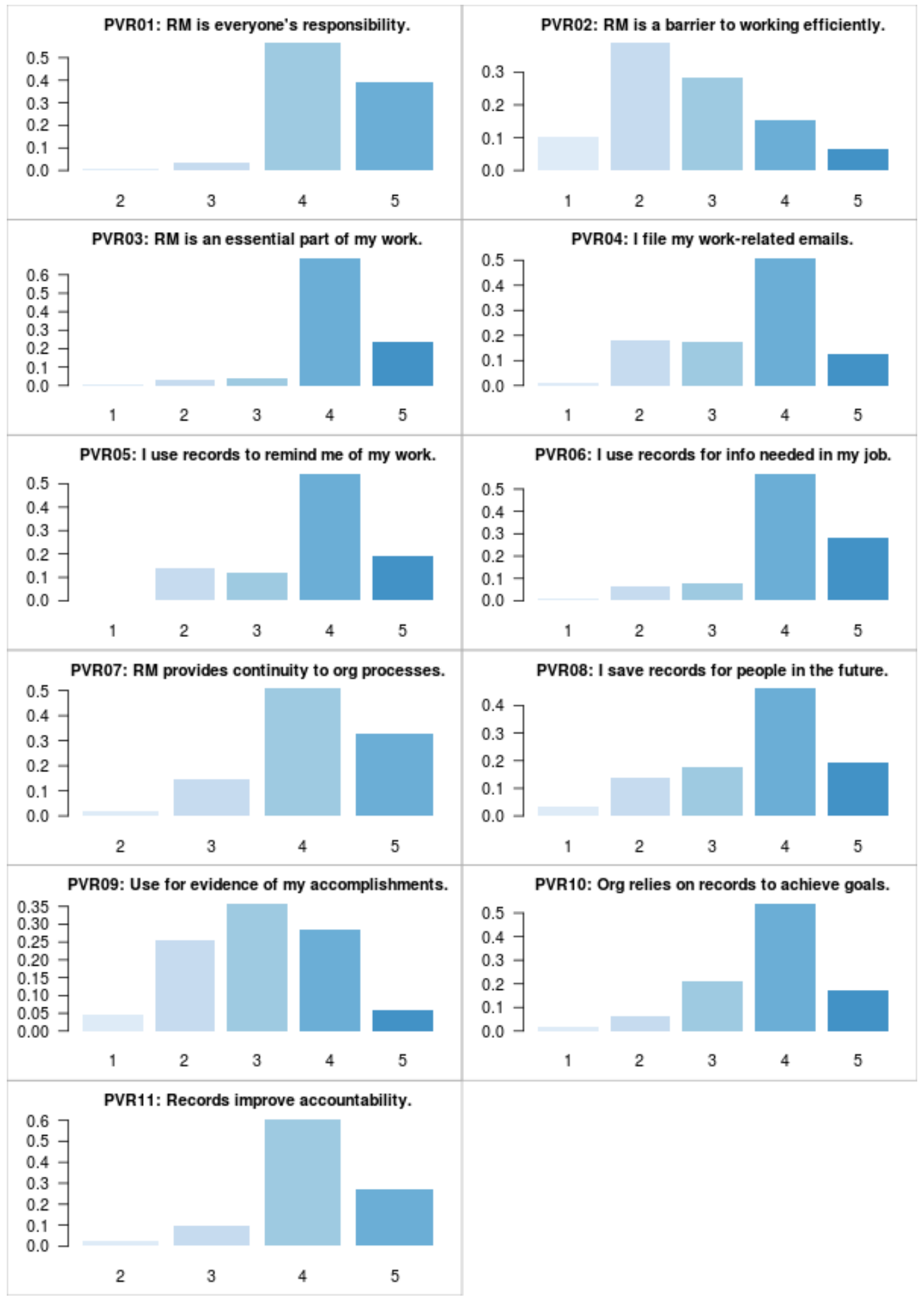

Figure E.5: Bar chart of survey responses: perceived value of records measurement items 


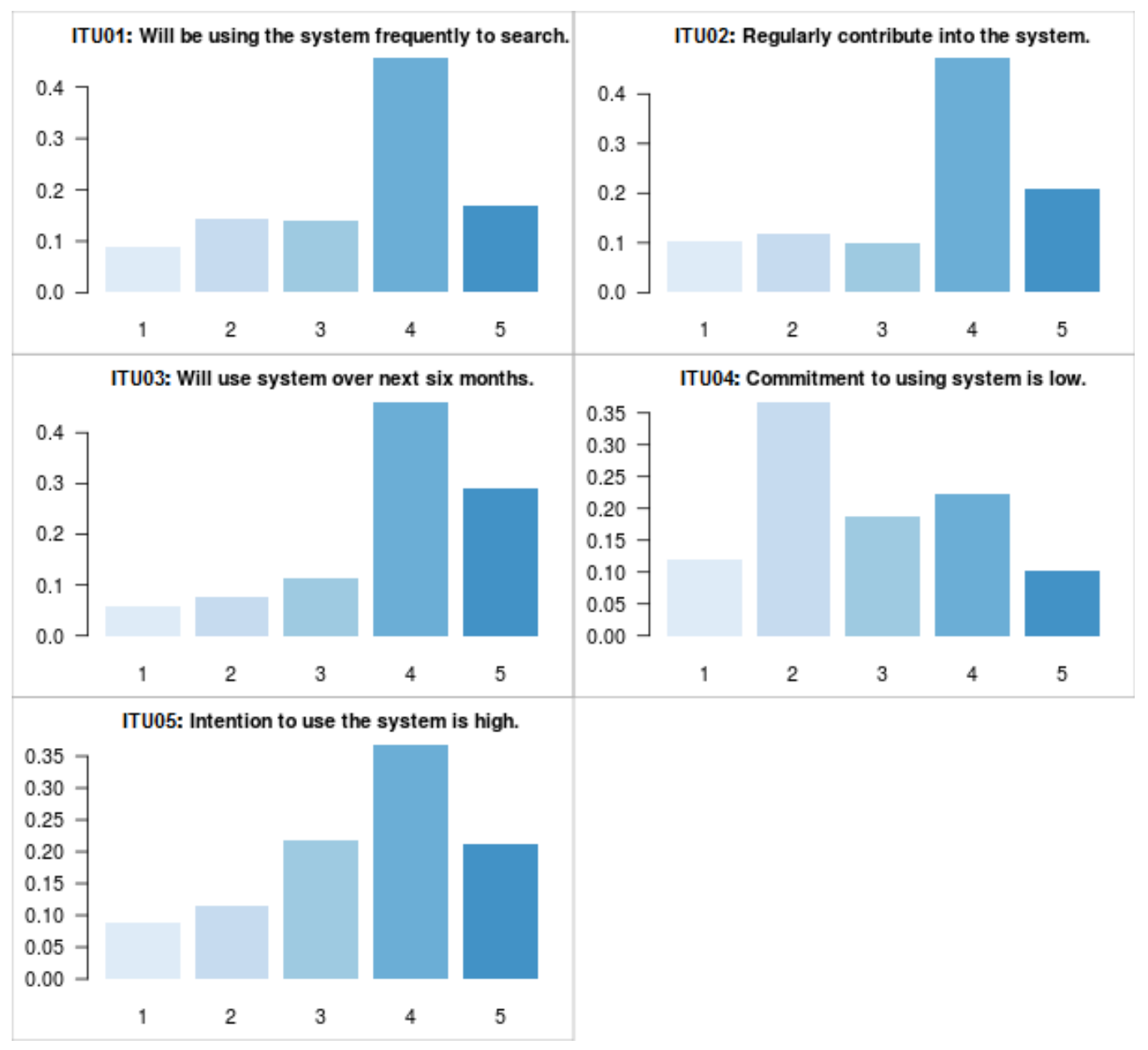

Figure E.6: Bar chart of survey responses: intention to use measurement items 


\section{Appendix F}

\section{Bootstrap Validation}

The bootstrap validation consisted of a run using a resample rate of 200 . The tables on the following pages provide the bootstrap results and significance levels for the following aspects of the model:

- Outer Model Validation

- Measurement Item Weights

- Measurement Item Loadings

- Inner Model Validation

- Paths (as specified in the model)

- Coefficients of Determination $\left(R^{2}\right)$

Each table presents the original value that emerged from the initial PLS-PM analysis, and then compares that value to the Mean Bootstrapped value (Mean.Boot) from the bootstrapping sample. The standard error (Std.Error) is then presented to provide an indication of the standard deviation of the mean. Finally, the lower percentiles (perc.025) and upper percentiles (perc. 975) of the $95 \%$ bootstrap confidence intervals are provided to indicate the significance. 


\section{WEIGHTS}

\begin{tabular}{lccccc} 
& Original & Mean.Boot & Std.Error & perc.025 & perc.975 \\
\hline SI01 & 0.3644 & 0.3577 & 0.0513 & 0.2491 & 0.4490 \\
SI02 & 0.3930 & 0.3917 & 0.0485 & 0.2979 & 0.4860 \\
SI05 & 0.4920 & 0.4973 & 0.0703 & 0.3711 & 0.6380 \\
SI06 & 0.2472 & 0.2279 & 0.1379 & $-0.0730^{*}$ & 0.4660 \\
SI07 & 0.4063 & 0.3425 & 0.2307 & $-0.1974^{*}$ & 0.8040 \\
SI08 & 0.0300 & 0.0558 & 0.2739 & $-0.6618^{*}$ & 0.5700 \\
SI09 & 0.0975 & 0.1038 & 0.2283 & $-0.5138^{*}$ & 0.5390 \\
SI10 & 0.4185 & 0.3686 & 0.2870 & $-0.3420^{*}$ & 0.9270 \\
PVR03 & 0.2682 & 0.2643 & 0.0374 & 0.2046 & 0.3440 \\
PVR06 & 0.2427 & 0.2382 & 0.0385 & 0.1418 & 0.2970 \\
PVR07 & 0.1826 & 0.1773 & 0.0499 & 0.0830 & 0.2680 \\
PVR08 & 0.4178 & 0.4216 & 0.0650 & 0.3121 & 0.5470 \\
PVR11 & 0.2649 & 0.2650 & 0.0445 & 0.1846 & 0.3650 \\
EE01 & 0.1896 & 0.1877 & 0.0117 & 0.1660 & 0.2110 \\
EE02 & 0.1906 & 0.1925 & 0.0118 & 0.1675 & 0.2150 \\
EE03 & 0.2354 & 0.2329 & 0.0139 & 0.2099 & 0.2650 \\
EE04 & 0.1748 & 0.1731 & 0.0134 & 0.1455 & 0.1970 \\
EE06 & 0.1863 & 0.1906 & 0.0162 & 0.1642 & 0.2290 \\
EE08 & 0.1924 & 0.1918 & 0.0143 & 0.1607 & 0.2180 \\
PE01 & 0.2281 & 0.2298 & 0.0173 & 0.1983 & 0.2660 \\
PE02 & 0.2377 & 0.2385 & 0.0130 & 0.2163 & 0.2640 \\
PE03 & 0.2329 & 0.2333 & 0.0128 & 0.2118 & 0.2600 \\
PE06 & 0.1482 & 0.1477 & 0.0223 & 0.0988 & 0.1880 \\
PE07 & 0.1774 & 0.1767 & 0.0158 & 0.1435 & 0.2070 \\
PE10 & 0.1890 & 0.1878 & 0.0136 & 0.1602 & 0.2150 \\
ITU01 & 0.2204 & 0.2230 & 0.0130 & 0.2010 & 0.2490 \\
ITU02 & 0.2171 & 0.2173 & 0.0100 & 0.1953 & 0.2360 \\
ITU03 & 0.2076 & 0.2102 & 0.0115 & 0.1877 & 0.2330 \\
ITU04 & 0.2326 & 0.2304 & 0.0142 & 0.1997 & 0.2570 \\
ITU05 & 0.2529 & 0.2512 & 0.0102 & 0.2331 & 0.2730 \\
\hline *Not significant (corresponding & to the Power construct) & \\
& & & & &
\end{tabular}

Table F.1: WEIGHTS: bootstrapping analysis focusing on the weights of the outer model measurement items. 


\section{LOADINGS}

\begin{tabular}{lccccc} 
& Original & Mean.Boot & Std.Error & perc.025 & perc.975 \\
\hline SI01 & 0.8180 & 0.8100 & 0.0462 & 0.7075 & 0.8880 \\
SI02 & 0.8450 & 0.8390 & 0.0419 & 0.7446 & 0.9080 \\
SI05 & 0.7520 & 0.7570 & 0.0470 & 0.6696 & 0.8410 \\
SI06 & 0.8570 & 0.7900 & 0.1748 & 0.2167 & 0.9200 \\
SI07 & 0.8730 & 0.7920 & 0.1907 & 0.1689 & 0.9450 \\
SI08 & 0.7550 & 0.7100 & 0.2119 & $0.0241^{*}$ & 0.9160 \\
SI09 & 0.7810 & 0.7290 & 0.1943 & 0.1376 & 0.9070 \\
SI10 & 0.8000 & 0.7110 & 0.2354 & 0.0261 & 0.9360 \\
PVR03 & 0.6980 & 0.6900 & 0.0507 & 0.5849 & 0.7780 \\
PVR06 & 0.7720 & 0.7640 & 0.0525 & 0.6411 & 0.8460 \\
PVR07 & 0.6710 & 0.6680 & 0.0682 & 0.4861 & 0.7720 \\
PVR08 & 0.7650 & 0.7720 & 0.0363 & 0.7016 & 0.8380 \\
PVR11 & 0.6920 & 0.6960 & 0.0592 & 0.5745 & 0.7940 \\
EE01 & 0.8460 & 0.8450 & 0.0218 & 0.8017 & 0.8860 \\
EE02 & 0.8920 & 0.8930 & 0.0185 & 0.8523 & 0.9250 \\
EE03 & 0.8900 & 0.8880 & 0.0175 & 0.8501 & 0.9170 \\
EE04 & 0.8550 & 0.8550 & 0.0230 & 0.8067 & 0.8960 \\
EE06 & 0.8050 & 0.8070 & 0.0281 & 0.7462 & 0.8570 \\
EE08 & 0.8340 & 0.8360 & 0.0262 & 0.7849 & 0.8810 \\
PE01 & 0.8150 & 0.8140 & 0.0228 & 0.7657 & 0.8540 \\
PE02 & 0.8900 & 0.8910 & 0.0190 & 0.8511 & 0.9210 \\
PE03 & 0.9040 & 0.9030 & 0.0107 & 0.8832 & 0.9230 \\
PE06 & 0.6620 & 0.6590 & 0.0625 & 0.5213 & 0.7570 \\
PE07 & 0.7800 & 0.7760 & 0.0389 & 0.6900 & 0.8360 \\
PE10 & 0.8220 & 0.8170 & 0.0280 & 0.7577 & 0.8630 \\
ITU01 & 0.8320 & 0.8310 & 0.0318 & 0.7671 & 0.8900 \\
ITU02 & 0.8940 & 0.8920 & 0.0172 & 0.8620 & 0.9270 \\
ITU03 & 0.9110 & 0.9110 & 0.0149 & 0.8808 & 0.9400 \\
ITU04 & 0.8550 & 0.8510 & 0.0312 & 0.7818 & 0.8970 \\
ITU05 & 0.9280 & 0.9270 & 0.0108 & 0.9038 & 0.9460 \\
\hline$*$ N0t signi & & &
\end{tabular}

*Not significant at the $95 \%$ confidence level.

Table F.2: LOADINGS: bootstrapping analysis focusing on the loadings of the outer model measurement items. 
PATHS (specified in the model)

\begin{tabular}{cccccc} 
& Original & Mean.Boot & Std.Error & perc.025 & perc.975 \\
\hline$S I \rightarrow P E$ & 0.1602 & 0.1592 & 0.0456 & 0.0674 & 0.2448 \\
$S I \rightarrow I T U$ & 0.2228 & 0.2221 & 0.0642 & 0.0902 & 0.3329 \\
$P O W \rightarrow E E$ & 0.1109 & 0.1100 & 0.1131 & $-0.2271^{*}$ & 0.2499 \\
$P O W \rightarrow I T U$ & -0.0653 & 0.0574 & 0.0642 & $-0.1907^{*}$ & 0.0635 \\
$P V R \rightarrow P E$ & 0.0776 & 0.0885 & 0.0500 & $-0.0116^{*}$ & 0.1807 \\
$P V R \rightarrow I T U$ & 0.4062 & 0.4093 & 0.0627 & 0.2930 & 0.5168 \\
$E E \rightarrow P E$ & 0.6871 & 0.6830 & 0.0409 & 0.5986 & 0.7529 \\
$E E \rightarrow I T U$ & 0.3032 & 0.3184 & 0.0766 & 0.1812 & 0.4804 \\
$P E \rightarrow I T U$ & 0.0986 & 0.0787 & 0.0914 & $-0.1066^{*}$ & 0.2599 \\
\hline
\end{tabular}

*Not significant

Table F.3: PATHS: bootstrapping analysis focusing on the inner model path coefficients.

\begin{tabular}{lccccc}
\multicolumn{2}{c}{ RSQ } & $(\mathrm{R}-\mathrm{Squared})$ \\
& Original & Mean.Boot & Std.Error & perc.025 & perc. 975 \\
\hline EE & 0.012 & 0.025 & 0.019 & $0.000^{*}$ & 0.073 \\
PE & 0.586 & 0.591 & 0.040 & 0.510 & 0.662 \\
ITU & 0.516 & 0.538 & 0.053 & 0.435 & 0.637 \\
\hline
\end{tabular}

*Not significant

Table F.4: R-Squared: bootstrapping analysis focusing on the coefficient of determination $\left(R^{2}\right)$ for the inner model's endogenous variables. 


\section{Bibliography}

Abdi, H. (2003). Multivariate analysis. Encyclopedia for research methods for the social sciences. Thousand Oaks: Sage, 699-702. Retrieved February 15, 2014, from http://www.utd.edu/ Herve/Abdi-MultivariateAnalysispretty.pdf

Abdi, H. \& Williams, L. (2010). Principal component analysis. Wiley Interdisciplinary Reviews: Computational Statistics, 2(4), 433-459.

Ackman, D. (2002, June 26). The WorldCom we hardly knew [Forbes.com]. Retrieved April 24, 2011, from http://www.forbes.com/2002/06/26/ 0626topnews.html

Adams, D. A., Nelson, R. R., \& Todd, P. A. (1992, June). Perceived usefulness, ease of use, and usage of information technology: a replication. MIS Quarterly, 16(2), 227-247.

Agourram, H. \& Robson, B. (2006). Defining information systems success in Canada. Information Management \& Computer Security, 14(4), 300. doi:10.1108/09685220610690781

Ajzen, I. (1991). The theory of planned behavior. Organizational Behavior and Human Decision Processes, 50(2), 179-211.

Archives New Zealand. (2009). Digital continuity action plan: managing information for public sector efficiency. Archives New Zealand. Wellington, N.Z.

Archives New Zealand. (2011). Government Digital Archive Programme [Archives New Zealand: Advice on Records \& Archiving]. Retrieved July 2, 2011, from http://archives.govt.nz/advice/government-digitalarchive-programme

Ashton, C. \& Reed, B. (2011, December 12). Reinventing records classification. Information $\&$ Records Management Annual (iRMA).

Atherton, A. \& Elsmore, P. (2007). Structuring qualitative enquiry in management and organization research: a dialogue on the merits of using 
software for qualitative data analysis. Qualitative Research in Organizations and Management: An International Journal, 2(1), 62-77.

Atherton, J. (1985). From life cycle to continuum: some thoughts on the records management-archives relationship. Archivaria, 21, 43-51.

Auer, T. (1998, September). Quality of IS use. European Journal of Information Systems, 7(3), 192-201. doi:10.1038/sj.ejis.3000305

Bagozzi, R. P. (1982). A field investigation of causal relations among cognitions, affect, intentions, and behavior. Journal of Marketing Research, 19(4), 562-583. doi:10.2307/3151727

Bailer-Jones, D. M. (2003, March). When scientific models represent. International Studies in the Philosophy of Science, 17(1), 59.

Bailey, S. (2007). Taking the road less travelled by: the future of the archive and records management profession in the digital age. Journal of the Society of Archivists, 28(2), 117-124.

Bailey, S. (2009). Forget electronic records management, it's automated records management that we desperately need. Records Management Journal, 19(2), 91-97.

Barki, H. \& Hartwick, J. (1994). Measuring user participation, user involvement, and user attitude. MIS Quarterly, 59-82.

Barkin Gary, W. \& Stephen, R. (1977). An investigation of information system utilization. Information 85 Management, 1(1), 35-45.

Barkin, S. \& Dickson, G. (1977). An investigation of information system utilization. Information \& Management, 1(1), 35-45.

Benbasat, I. \& Barki, H. (2007). Quo vadis TAM? Journal of the Association for Information Systems, 8(4), 7.

Benfell, P. (2002). An integrated approach to managing electronic records. Records Management Journal, 12(3), 94-97.

Bhaskar, R. (1975). A realist theory of science. Leeds, England: Leeds Books Limited.

Bohrnstedt, G. W. (1970). Reliability and validity assessment in attitude measurement. In G. Summers (Ed.), Attitude measurement (pp. 80-99). Chicago: Rand McNally.

Bollen, K. \& Lennox, R. (1991, September). Conventional wisdom on measurement: a structural equation perspective. Psychological Bulletin, 110(2), 305-314. doi:http: / / dx. doi.org. helicon .vuw . ac.nz / 10 .1037 / 0033 2909.110.2.305

Boudreau, M. C., Gefen, D., \& Straub, D. W. (2001). Validation in information systems research: a state-of-the-art assessment. MIS Quarterly, 1-16. 
Boztepe, S. (2007). User value: competing theories and models. International Journal of Design, 1(2), 55-63.

Braun, V. \& Clarke, V. (2006). Using thematic analysis in psychology. Qualitative Research in Psychology, 3(2), 77-101.

Brown, S. A., Massey, A. P., Montoya-Weiss, M. M., \& Burkman, J. R. (2002). Do I really have to? User acceptance of mandated technology. European Journal of Information Systems, 11(4), 283-295.

Bruce, H. (2005, April). Personal anticipated information need. Information Research, 10(3).

Burrell, G. \& Morgan, G. (1979). Sociological paradigms and organisational analysis. London: Heinemann.

Burton-Jones, A. \& Straub, D. W. (2006). Reconceptualizing system usage: an approach and empirical test. Information Systems Research, 17(3), 228.

Byrne, M. (2001a). Data analysis strategies for qualitative research. AORN, $74(6), 904-905$.

Byrne, M. (2001b). Interviewing as a data collection method. AORN, $74(2)$, $233-235$.

Cain, P. (2002). Model requirements for the management of electronic records (MoReq): a critical evaluation. Records Management Journal, 12(1), $14-18$.

Chang, S.-J., van Witteloostuijn, A., \& Eden, L. (2010, February). From the editors: common method variance in international business research. Journal of International Business Studies, 41(2), 178-184. doi:10.1057/ jibs. 2009.88

Chang, T.-Z. (2013, June). Strategies for improving data reliability for online surveys: a case study. International Journal of Electronic Commerce Studies, 121-130. doi:10.7903/ijecs.1121

Chin, W. W. (1998a). Commentary: issues and opinion on structural equation modeling. MIS Quarterly, vii-xvi.

Chin, W. W. (1998b). The partial least squares approach for structural equation modeling. In Modern methods for business research (pp. 295336). Methodology for business and management. Mahwah, NJ, US: Lawrence Erlbaum Associates Publishers.

Chin, W. W. (2010a). Bootstrap cross-validation indices for PLS path model assessment. In Handbook of partial least squares (pp. 83-97). Springer Handbooks of Computational Statistics. Springer: Handbooks of Computational Statistics. 
Chin, W. W. (2010b). How to write up and report PLS analyses. In Handbook of partial least squares (pp. 655-690). Springer: Handbooks of Computational Statistics.

Chin, W. W., Gopal, A., \& Salisbury, W. D. (1997). Advancing the theory of adaptive structuration: the development of a scale to measure faithfulness of appropriation. Information Systems Research, 8, 342-367.

Choo, C. W., Bergeron, P., Detlor, B., \& Heaton, L. (2008). Information culture and information use: an exploratory study of three organizations. Journal of the American Society for Information Science and Technology, 59(5), 792-804. doi:10.1002/asi.20797

Churchill Jr, G. A. (1979). A paradigm for developing better measures of marketing constructs. Journal of Marketing Research, 16, 64-73. doi:10. $2307 / 3150876$

Clever recordkeeping metadata project - final report. (2007, August). Monash University. Melbourne, Australia.

Collopy, F. (1996). Biases in retrospective self-reports of time use: an empirical study of computer users. Management Science, 42(5), 758-767.

Compeau, D. R. \& Higgins, C. A. (1995). Computer self-efficacy: development of a measure and initial test. MIS Quarterly, 19(2), 189-211. doi:10. $2307 / 249688$

Compeau, D. R., Higgins, C. A., \& Huff, S. (1999, June 1). Social cognitive theory and individual reactions to computing technology: a longitudinal study. MIS Quarterly, 23(2), 145-158. doi:10.2307/249749

Cook, T. (1994). Electronic records, paper minds: the revolution in information management and archives in the post-custodial and post-modernist era. Archives and Manuscripts, 22(2), 300-328.

Cook, T. (1997). The impact of David Bearman on modern archival thinking: an essay of personal reflection and critique. Archives and Museum Informatics, $11(1), 15-37$.

Corbin, J. \& Strauss, A. (2008). Basics of qualitative research: techniques and procedures for developing grounded theory. London, UK: Sage Publications, Inc.

Creswell, J. W. (2008). Research design: qualitative, quantitative, and mixed methods approaches (3rd ed.). Los Angeles, CA: Sage Publications, Inc.

CRKM Project. (2011, April 3). (CRKM) clever recordkeeping metadata project. Retrieved from http://www.infotech.monash.edu.au/research/ groups $/ \mathrm{rcrg} / \mathrm{crkm} /$

Cullen, C. T., Hirtle, P. B., Levy, D., Lynch, C. A., \& Rothenberg, J. (2000, May). Authenticity in a digital environment. Washington, D.C.: Council 
on Library and Information Resources. Retrieved from http://www.clir. org

Cunningham, A. (2011). Good digital records don't just "happen": embedding digital recordkeeping as an organic component of business processes and systems. Archivaria, 71, 21-34.

Davis, F. D. (1986). A technology acceptance model for empirically testing new end-user information systems: theory and results (PhD Thesis, Massachusetts Institute of Technology, Sloan School of Management, Massachusetts). Retrieved from http://hdl.handle.net/1721.1/15192

Davis, F. D. (1989). Perceived usefulness, perceived ease of use, and user acceptance of information technology. MIS Quarterly, 13(3), 319-340.

Davis, F. D., Bagozzi, R. P., \& Warshaw, P. R. (1989). User acceptance of computer technology: a comparison of two theoretical models. Management Science, 35(8), 982-1003.

Deetz, S. (1996). Describing differences in approaches to organization science: rethinking Burrell and Morgan and their legacy. Organization Science, $7(2), 191-207$.

DeLone, W. H. \& McLean, E. R. (1992, March). Information systems success: the quest for the dependent variable. Information Systems Research, 3(1), 60-95. doi:10.1287/isre.3.1.60

DeLone, W. H. \& McLean, E. R. (2003). The DeLone and McLean model of information systems success: a ten-year update. Journal of Management Information Systems, 19(4), 9-30.

Dictionary.com. (2014). Dictionary.com Unabridged [Random House, Inc.]. Retrieved March 26, 2014, from http://dictionary.reference.com/browse/ useful

Diez, R. (2002). A glossary for multilevel analysis. Journal of Epidemiology and Community Health, 56(8), 588.

Digital Recordkeeping Standard. (2010, August). Retrieved from http:// archives.govt.nz/advice/continuum-resource-kit/continuum-publications$\mathrm{html} / \mathrm{s} 5$-digital-recordkeeping-standard

Dillman, D. A. (2007). Mail and internet surveys: the tailored design method2007 update with new internet, visual, and mixed-mode guide (2nd ed.). Hoboken, New Jersey: John Wiley \& Sons.

Dillman, D. A. (2009). Internet, mail, and mixed-mode surveys: the tailored design method (3rd ed.). Hoboken, New Jersey: Wiley \& Sons.

Dillon, W. R., Madden, T. J., \& Firtle, N. H. (1990). Marketing research in a marketing environment (3rd ed.). Burr Ridge, IL: Irwin Homewood. 
Dishaw, M. T. \& Strong, D. M. (1999, July). Extending the technology acceptance model with task-technology fit constructs. Information 8 Management, 36(1), 9-21. doi:10.1016/S0378-7206(98)00101-3

DoD 5015.02-STD. (2007, April 25). DoD Standard - Electronic Records Management Software Applications Design Criteria Standard. Department of Defense (USA).

Dudding, A. \& Mayo, K. (2010a, July 23). Government recordkeeping survey report 2010 - local authorities (No. NZ200187). Archives New Zealand. Wellington, New Zealand.

Dudding, A. \& Mayo, K. (2010b, July 23). Government recordkeeping survey report 2010 - public offices (No. NZ200187). Archives New Zealand. Wellington, New Zealand.

Duranti, L. (1996, November). Archives as a place. Archives and Manuscripts, $24(2), 242-255$.

Duranti, L. (2010). Concepts and principles for the management of electronic records, or records management theory is archival diplomatics (republished - 1999). Records Management Journal, 20(1), 78-95.

Duranti, L. (2012). Records in the cloud: towards InterPARES-trust. Fondazione Rinascimento Digitale. The University of British Columbia, Canada. Retrieved July 6, 2014, from http://93.63.166.138:8080/ dspace/handle/2012/97

Duranti, L. \& Blanchette, J. (2004). The authenticity of electronic records: the InterPARES approach. In Proceedings of ISET 2004 archiving conference (pp. 215-220).

Evans, J. \& Lindberg, L. (2004). Describing and analyzing the recordkeeping capabilities of metadata sets. In Proceedings of the 2004 international conference on dublin core and metadata applications: metadata across languages and cultures (p. 9).

Evans, J., McKemmish, S., \& Bhoday, K. (2006, March). Create once, use many times: the clever use of recordkeeping metadata for multiple archival purposes. Archival Science, 5(1), 17-42. doi:10.1007/s10502005-4625-X

Evans, J., Reed, B., \& McKemmish, S. (2008). Interoperable data: sustainable frameworks for creating and managing recordkeeping metadata. Records Management Journal, 18(2), 115-129.

Faiks, A. \& Hyland, N. (2000). Gaining user insight: a case study illustrating the card sort technique. College \& research libraries, 61 (4), 349-357. Retrieved February 18, 2013, from http://crl.acrl.org/content/61/4/ 349.short 
Fereday, J. \& Muir-Cochrane, E. (2008). Demonstrating rigor using thematic analysis: a hybrid approach of inductive and deductive coding and theme development. International Journal of Qualitative Methods, 5(1), 80-92.

Fidock, J. \& Carroll, J. (2009, December 2). Combining variance and process research approaches to understand system use. In Combining variance and process research approaches (pp. 235-245). 20th Australasian Conference on Information Systems. Melbourne, Australia.

Fishbein, M. \& Ajzen, I. (1975). Belief, attitude, intention, and behavior: an introduction to theory and research. Reading, MA: Addison-Wesley Pub. Co.

Foddy, W. (1998, August 1). An empirical evaluation of in-depth probes used to pretest survey questions. Sociological Methods \& Research, 27(1), 103-133. doi:10.1177/0049124198027001003

Fresko, M. (2010, September). MoReq2010 - a new world standard for compliance \& best practice information management for all sectors. Information and Records Management Society Bulletin, (157), 18-20.

Garver, M. \& Mentzer, J. (1999). Logistics research methods: employing structural equation modeling to test for construct validity. Journal of Business Logistics, 20, 33-58.

Giddens, A. (1984). The constitution of society. Berkeley: University of California Press.

Gilliland, A., Rouche, N., Lindberg, L., \& Evans, J. (2005). Towards a 21st century metadata infrastructure supporting the creation, preservation and use of trustworthy records: developing the InterPARES 2 metadata schema registry. Archival Science, 5(1), 43-78. doi:10.1007/s10502-0059000-4

Ginzberg, M. J. (1981, June). Key recurrent issues in the MIS implementation process. MIS Quarterly, 5(2), 47-59. doi:10.2307/249223

Goodhue, D. L. (2007, April). Comment on Benbasat and Barki's "Quo Vadis TAM" article. Journal of the Association for Information Systems, 8(4), 220-222.

Goodhue, D. L. \& Thompson, R. L. (1995, June). Task-technology fit and individual performance. MIS Quarterly, 19(2), 213-236. doi:10.2307/ 249689

Greene, J. C. \& Caracelli, V. J. (1997). Defining and describing the paradigm issue in mixed-method evaluation. New Directions for Evaluation, 1997(74), $5-17$. 
Greene, J. C., Caracelli, V. J., \& Graham, W. F. (1989). Toward a conceptual framework for mixed-method evaluation designs. Educational Evaluation and Policy Analysis, 11 (3), 255-274.

Gregor, S. (2006, September). The nature of theory in information systems. MIS Quarterly, 30(3), 611-642.

Hackbarth, G., Grover, V., \& Yi, M. Y. (2003). Computer playfulness and anxiety: positive and negative mediators of the system experience effect on perceived ease of use. Information $\&$ management, 40(3), 221-232.

Haenlein, M. \& Kaplan, A. M. (2004). A beginner's guide to partial least squares analysis. Understanding Statistics, 3(4), 283-297.

Hanafi, M. (2007, July 1). PLS path modelling: computation of latent variables with the estimation mode b. Computational Statistics, 22(2), 275-292. doi:10.1007/s00180-007-0042-3

Hardgrave, B. C. \& Johnson, R. A. (2003). Toward an information systems development acceptance model: the case of object-oriented systems development. IEEE Transactions on Engineering Management, 50(3), 322-336.

Harries, S. (2009). Managing records, making knowledge and good governance. Records Management Journal, 19(1), 16-25.

Hasan, R., Sion, R., \& Winslett, M. (2007). Introducing secure provenance: problems and challenges. In Proceedings of the $200^{7}$ ACM workshop on storage security and survivability (p. 18).

Helberg, C. (1995). Pitfalls of data analysis (or how to avoid lies and damned lies). (Vol. 5, p. 2005). Third international applied statistics in industry. Dallas, TX.

Hendrickson, A. R., Massey, P. D., \& Cronan, T. P. (1993, June). On the test-retest reliability of perceived usefulness and perceived ease of use scales. MIS Quarterly, 17(2), 227-230.

Henseler, J. \& Sarstedt, M. (2013, April 1). Goodness-of-fit indices for partial least squares path modeling. Computational Statistics, 28(2), 565-580. doi:10.1007/s00180-012-0317-1

Hinkin, T. (1998). A brief tutorial on the development of measures for use in survey questionnaires. Organizational Research Methods, 1, 104.

Hinkle, V. (2008, October). Card-sorting: what you need to know about analyzing and interpreting card sorting results. Software Usability Research Laboratory, Wichita State University. Usability News, 10(2). Retrieved February 19, 2013, from http://tinyurl.com/logczk5

Hoe, S. (2008). Issues and procedures in adopting structural equation modeling technique. Journal of applied quantitative methods, 3(1), 76-83. 
Holbrook, M. (Ed.). (1999). Consumer value: a framework for analysis and research. London: Routledge.

Holtzman, M., Venuti, E., \& Fonfeder, R. (2003, April 3). Enron and the raptors [The CPA journal]. Retrieved April 25, 2011, from http://www. nysscpa.org/cpajournal/2003/0403/features/f042403.htm

Hox, J. \& Bechger, T. (1998). An introduction to structural equation modeling. Family Science Review, 11, 354-373.

Hunt, S. D., Sparkman Jr., R. D., \& Wilcox, J. B. (1982, May). The pretest in survey research: issues and preliminary findings. Journal of Marketing Research (JMR), 19(2), 269-273.

Hussein, R., Karim, N. S. A., \& Selamat, M. H. (2007). The impact of technological factors on information systems success in the electronicgovernment context. Business Process Management Journal, 13(5), 613.

Ince, D. C., Hatton, L., \& Graham-Cumming, J. (2012, February 22). The case for open computer programs. Nature, 482(7386), 485-488. doi:10. 1038/nature10836

InterPARES Project. (2011). InterPARES project. Retrieved April 3, 2011, from http://www.interpares.org/

ISO 15489.1. (2002). ISO Standard: Records Management - Part 1: General. Standards Australia.

ISO 23081-1. (2006). ISO 23081-1 Information and Documentation-Records Management Processes-Metadata for Records-Part 1: Principles. (2006). International Organization for Standardization.

Jackson, D. N. \& Paunonen, S. V. (1985). Construct validity and the predictability of behavior. Journal of Personality and Social Psychology, 49, 554-570.

Johnston, G. P. \& Bowen, D. V. (2005, December 1). The benefits of electronic records management systems: a general review of published and some unpublished cases. Records Management Journal, 15(3), 131-140. doi:10. $1108 / 09565690510632319$

Jones, M. \& Karsten, H. (2008, March). Giddens's structuration theory and information systems research. MIS Quarterly, 32(1), 127-157.

Jones, M., Orlikowski, W. J., \& Munir, K. (2004). Structuration theory and information systems: a critical reappraisal. In J. Mingers \& L. Willcocks (Eds.), Social theory and philosophy for information systems (pp. 297328). Chichester, UK: John Wiley \& Sons Ltd. 
Jones, S. (2012, August). eGovernment document management system: a case analysis of risk and reward. International Journal of Information Management, 32(4), 396-400. doi:10.1016/j.ijinfomgt.2012.04.002

Joseph, P. (2008). EDRMS 101: the basics. iRMA: Information and Records Management Annual, 9-26.

Joseph, P., Debowski, S., \& Goldschmidt, P. (2012, March 23). Paradigm shifts in recordkeeping responsibilities: implications for ISO 15489's implementation. Records Management Journal, 22(1), 57-75. doi:10. 1108/09565691211222108

Joseph, P., Debowski, S., \& Goldschmidt, P. (2013). Search behaviour in electronic document and records management systems: an exploratory investigation and model. Information Research - an International Electronic Journal, 18(1).

Karahanna, E. \& Straub, D. W. (1999, April 5). The psychological origins of perceived usefulness and ease-of-use. Information $\&$ Management, 35 (4), 237-250. doi:10.1016/S0378-7206(98)00096-2

Karahanna, E., Straub, D. W., \& Chervany, N. L. (1999, June). Information technology adoption across time: a cross-sectional comparison of preadoption and post-adoption beliefs. MIS Quarterly, 23(2), 183-213.

Kettunen, K. \& Henttonen, P. (2010). Missing in action? content of records management metadata in real life. Library $\&$ Information Science Research, 32(1), 43-52.

Kim, S. S. \& Malhotra, N. (2005, May). A longitudinal model of continued IS use: an integrative view of four mechanisms underlying postadoption phenomena. Management Science, 51(5), 741-755.

Klein, J., Connell, C., \& Jasimuddin, S. (2007, May). Who needs memory? The case for the Markovian organisation. Knowledge Management Research \& Practice, 5 (2), 110-116. doi:10.1057/palgrave.kmrp.8500130

Klein, K. J. \& Kozlowski, S. W. (2000). From micro to meso: critical steps in conceptualizing and conducting multilevel research. Organizational Research Methods, 3(3), 211-236.

Kling, R. (1980). Social analyses of computing: theoretical perspectives in recent empirical research. ACM Computing Surveys (CSUR), 12(1), $61-110$.

Lappin, J. (2010). What will be the next records management orthodoxy? Records Management Journal, 20(3), 252-264.

Lappin, J. (2011a, May 6). How MoReq 2010 differs from previous electronic records management (ERM) system specifications [Thinking records]. Retrieved October 5, 2011, from http://thinkingrecords.co.uk/2011/ 
05 / 06 / how - moreq- 2010 - differs - from- previous - electronic- recordsmanagement-erm-system-specifications/

Lappin, J. (2011b, October 19). Is SharePoint a records management system? podcast [Thinking records]. Retrieved 2014, from http://thinkingrecords. co . uk / 2011/10/19/is-sharepoint-a-records- management-systempodcast/

Lee, A. (2014, June 26). Keynote address. 18th Pacific Asia Conference on Information Systems (PACIS 2014), Chengdu, Sichuan, China.

Lee, Y., Kozar, K. A., \& Larsen, K. R. T. (2003, September). The technology acceptance model: past, present, and future. Communications of the Association for Information Systems, 12, 752-780.

Lewellen, M. (2013, July 26). Factors influencing employees' intention to contribute to an electronic recordkeeping system. New Zealand Information Systems Doctoral Consortium (NZISDC 2013). Auckland, New Zealand.

Lewellen, M., Hooper, V., \& Oliver, G. (2013, December 4). Factors influencing employees' intention to use an electronic recordkeeping system: development of a valid survey instrument. 24th Australasian Conference on Information Systems (ACIS 2013). Melbourne, Australia.

Lewellen, M., Hooper, V., \& Oliver, G. (2014a, December). The impact of knowledge interpretation and organizational context on the use of electronic recordkeeping systems. 25th Australasian Conference on Information Systems (ACIS 2014). Auckland University of Technology, Auckland, New Zealand: ACIS.

Lewellen, M., Hooper, V., \& Oliver, G. (2014b, June 24). Unpacking the subjective norm: applying structuration theory to traditional measures of social influence. (p. 11). 18th Pacific Asia Conference on Information Systems (PACIS 2014). Chengdu, China.

Lewis, B. R., Templeton, G. F., \& Byrd, T. A. (2005, December). A methodology for construct development in MIS research. European Journal of Information Systems, 14(4), 388-400. doi:http://dx.doi.org.helicon.vuw. ac.nz/10.1057/palgrave.ejis.3000552

Li, Z., Bao-wei, L., \& Ye-zhuang, T. (2007, August). Empirical study on organizational memory constructive factors. (pp. 1487-1492). 2007 international conference on management science \& engineering. Harbin, P.R. China: ICMSE. doi:10.1109/ICMSE.2007.4422053

Li, Z., YeZhuang, T., \& ZhongYing, Q. (2004). An empirical study on the impact of organizational memory on organizational performance in manufacturing companies. In The 37th annual Hawaii international 
conference on system sciences (p. 10). Koloa, Kauai, Hawaii: IEEE Computer Society.

Lips, M. \& Rapson, A. (2009, November). Emerging records management in 21st century New Zealand Government-Part 2. Victoria University of Wellington. Wellington, New Zealand.

Lips, M. \& Rapson, A. (2010). Exploring public recordkeeping behaviors in wiki-supported public consultation activities in the New Zealand public sector. In 2010 43rd Hawaii international conference on system sciences (pp. 1-10). HICSS. Koloa, Kauai, Hawaii: IEEE Computer Society. doi:10.1109/HICSS.2010.453

Lips, M., Rapson, A., \& Hooper, T. (2008, October 23). E-mail management knowledge and recordkeeping behaviours of New Zealand public servants. In 4 th international conference on e-government (p. 273). Melbourne, Australia.

Lucas Jr, H. C. (1978, June). Empirical evidence for a descriptive model of implementation. MIS Quarterly, 2(2), 27-42. doi:10.2307/248939

Lucas Jr, H. C. \& Spitler, V. K. (1999). Technology use and performance: a field study of broker workstations. Decision Sciences, 30(2), 291-311.

MacNeil, H. (2002). Providing grounds for trust II: the findings of the authenticity task force of InterPARES. Archivaria, 24-58.

MacNeil, H. (2004). Contemporary archival diplomatics as a method of inquiry: lessons learned from two research projects. Archival Science, 4(3), 199232.

Maguire, R. (2005). Lessons learned from implementing an electronic records management system. Records Management Journal, 15(3), 150-157. doi:10.1108/09565690510632337

Malhotra, M. \& Grover, V. (1998, July). An assessment of survey research in POM: from constructs to theory. Journal of Operations Management, 16 (4), 407-425. doi:10.1016/S0272-6963(98)00021-7

Martinko, M. J., Henry, J. W., \& Zmud, R. W. (1996). An attributional explanation of individual resistance to the introduction of information technologies in the workplace. Behaviour \& Information Technology, $15(5), 313-330$.

Mathieson, K. (1991). Predicting user intentions: comparing the technology acceptance model with the theory of planned behavior. Information systems research, 2(3), 173-191. doi:10.1287/isre.2.3.173

McCoy, S., Galletta, D. F., \& King, W. R. (2007). Applying TAM across cultures: the need for caution. European Journal of Information Systems, 16(1), 81-90. 
McKemmish, S. (1997). Yesterday, today and tomorrow: a continuum of responsibility. In Preserving yesterday, managing today and challenging tomorrow: proceedings 14th national convention RMAA, $199^{\text {ry }}$ (p. 19).

McKemmish, S., Acland, G., Ward, N., \& Reed, B. (1999). Describing records in context in the continuum: the australian recordkeeping metadata schema. Archivaria, 48, 3-43.

Melville, N., Kraemer, K., \& Gurbaxani, V. (2004). Information technology and organizational performance: an integrative model of IT business value. MIS Quarterly, 28(2), 283-322.

Misangyi, V. F., Elms, H., Greckhamer, T., \& Lepine, J. A. (2006, June). A new perspective on a fundamental debate: a multilevel approach to industry, corporate, and business unit effects. Strategic Management Journal, 27(6), 571-590.

Mishina, T., Yoshihama, S., \& Kudo, M. (2007). Fine-grained sticky provenance architecture for office documents. In Proceedings of the security 2nd international conference on advances in information and computer security (pp. 336-351).

Moore, G. C. \& Benbasat, I. (1991). Development of an instrument to measure the perceptions of adopting an information technology innovation. Information Systems Research, 2(3), 192-222.

Moreau, L., Groth, P., Miles, S., Vazquez-Salceda, J., Ibbotson, J., Jiang, S., ... Tan, V. (2008). The provenance of electronic data. Communications of the ACM, 51(4), 52-58.

MoReq2010. (2011, June 6). MoReq2010 - Modular Requirements for Records Systems. DLM Forum Foundation.

NARA. (2005). US National Archives and Records Administration Guidance on Managing Web Records: What Are the Characteristics of Trustworthy Records? Retrieved December 21, 2013, from http://www.archives.gov/ records-mgmt/policy/managing-web-records.html

National Archives of Australia. (2001). DIRKS: designing and implementing recordkeeping systems manual. Retrieved April 24, 2011, from http: //www.naa.gov.au/records-management/publications/dirks-manual. aspx

Nguyen, L. T. \& Swatman, P. (2007). EDMS, ERMS, ECMS or EDRMS: fighting through the acronyms towards a strategy for effective corporate records management. (pp. 790-800). 18th Australasian Conference on Information Systems. Toowoomba, Australia, 
Nguyen, L. T., Swatman, P., \& Fraunholz, B. (2008a). Australian public sector adoption of EDRMS: a preliminary survey. Australasia conference on information systems. Christchurch, New Zealand.

Nguyen, L. T., Swatman, P., \& Fraunholz, B. (2008b, June). Standing on the shoulders of giants: are ERP success factors relevant for EDRMS implementation? In eCollaboration: overcoming boundaries through multichannel interaction (pp. 523-536). The 21st Bled eConference. Bled, Slovenia.

Nguyen, L. T., Swatman, P., \& Fraunholz, B. (2009, December). EDRMS implementations in the Australian public sector. In 20th Australasian Conference on Information Systems (915(14)). EDRMS in the Australian Public Sector. Melbourne, Australia.

Oliver, G. (2008). Information culture: exploration of differing values and attitudes to information in organisations. Journal of Documentation, $64(3), 363$.

Oliver, G., Evans, J., Reed, B., \& Upward, F. (2009). Achieving the right balance: recordkeeping informatics, Part 1. InfoRMAA Quarterly: Official Bulletin of the Records Management Association of Australia, The, $25(4), 18-21$.

Oliver, G., Evans, J., Reed, B., \& Upward, F. (2010). Achieving the right balance: recordkeeping informatics, Part 2. InfoRMAA Quarterly: Official Bulletin of the Records Management Association of Australia, The, 26(1), 42-44, 52 .

Oliver, G. \& Foscarini, F. (2014). Records management and information culture: tackling the people problem. London: Facet Publishing.

Olivera, F. (2000). Memory systems in organizations: an empirical investigation of mechanisms for knowledge collection, storage and access. Journal of Management Studies, 37(6), 811-832.

Ong, C. S., Lai, J. Y., Wang, Y. M., \& Wang, S. W. (2005). An understanding of power issues influencing employees' acceptance of KMS: an empirical study of Taiwan semiconductor manufacturing companies. The 38th Hawaii International Conference on System Sciences. Koloa, Kauai, Hawaii.

Orlikowski, W. J. (1992). The duality of technology: rethinking the concept of technology in organizations. Organization Science, 3(3), 398-427.

Pederson, A. (2003). Understanding ourselves and others: Australian archivists and temperament. Archival Science, 3(3), 223-274. doi:10.1007/s10502004-4039-1 
Peter, J. P. (1981). Construct validity: a review of basic issues and marketing practices. Journal of Marketing Research, 18(2), 133-145.

Pinsonneault, A. \& Kraemer, K. L. (1993). Survey research methodology in management information systems: an assessment. Journal of Management Information Systems, $10(2), 105$.

Pittsburgh Project. (1993). Pittsburgh project. Retrieved September 2, 2010, from http://www.sis.pitt.edu/ bcallery/pgh/MainPage.htm

Podsakoff, P. M., MacKenzie, S. B., Lee, J.-Y., \& Podsakoff, N. P. (2003). Common method biases in behavioral research: a critical review of the literature and recommended remedies. Journal of applied psychology, $88(5), 879$.

Polfreman, M. \& Rajbhandari, S. (2008). MetaTools-Investigating metadata generation tools-final report.

Poole, M. S. \& DeSanctis, G. (2004). Structuration theory in information systems research: methods and controversies. In M. Whitman \& A. Woszczynski (Eds.), The Handbook of Information Systems Research (pp. 206-249). Idea Group Publishing.

Priest, H., Roberts, P., \& Woods, L. (2002). An overview of three different approaches to the interpretation of qualitative data. part 1: theoretical issues. Nurse Researcher, 10(1), 30.

Principles and Functional Requirements for Records in Electronic Office Environments. (2008). Principles and functional requirements for records in electronic office environments - module 2: guidelines and functional requirements for electronic records management systems. (2008). International Council on Archives.

Proctor, R. W. (2005). Methodology is more than research design and technology. Behavior Research Methods, 37(2), 197-201.

Public Records Act (NZ). (2005, April 20). Public records act 2005 of new zealand. (2005, April 20). Retrieved from http://www.legislation.govt. nz/act/public/2005/0040/latest/DLM345529.html

R Core Team. (2013). R: a language and environment for statistical computing. Vienna, Austria: R Foundation for Statistical Computing. Retrieved from http://www.R-project.org

Reed, B. (2008). Service-oriented architectures and recordkeeping. Records Management Journal, 18(1), 7. doi:10.1108/09565690810858488

Rugg, G. \& McGeorge, P. (2005). The sorting techniques: a tutorial paper on card sorts, picture sorts and item sorts. Expert Systems, 22(3), 94-107. doi:10.1111/j.1468-0394.2005.00300.x 
Salmoni, A. (2012, April 10). Open card sort analysis 101 [UX booth]. Retrieved February 19, 2013, from http://www.uxbooth.com/articles/ open-card-sort-analysis-101/

Sanchez, G. (2012a, October 3). 7+ ways to plot dendrograms in R [Gaston Sanchez]. Retrieved from http://gastonsanchez.com/blog/how-to/2012/ 10/03/Dendrograms.html

Sanchez, G. (2012b). Plsdepot: partial least squares (pls) data analysis methods. $\mathrm{R}$ package version 0.1.17. Retrieved from http://CRAN.R-project.org/ package $=$ plsdepot

Sanchez, G. (2013a). Overall comparison: PLS -vs- CSA [PLS modeling]. Retrieved 2014, from http://www.plsmodeling.com/extra/pls-csa

Sanchez, G. (2013b). PLS path modeling with r. eBook. Retrieved from http: //www.gastonsanchez.com/PLS_Path_Modeling_with_R.pdf

Sanchez, G., Trinchera, L., \& Russolillo, G. (2013). Plspm: tools for partial least squares path modeling (PLS-PM) (Version $\mathrm{R}$ package version 0.4.1). Retrieved from http://CRAN.R-project.org/package=plspm

Schepers, J. \& Wetzels, M. (2007). A meta-analysis of the technology acceptance model: investigating subjective norm and moderation effects. Information \& Management, 44(1), 90-103.

Schreiber, J., Nora, A., Stage, F., Barlow, E., \& King, J. (2006). Reporting structural equation modeling and confirmatory factor analysis results: a review. The Journal of Educational Research, 99(6), 323-338.

Schultze, U. \& Stabell, C. (2004, June). Knowing what you don't know? Discourses and contradictions in knowledge management research. Journal of Management Studies, 41(4), 549-573. doi:10.1111/j.1467-6486.2004. 00444.x

Schwarz, A. (2002, June). Managing with Markov. Harvard Magazine, 34-36.

Seddon, P. B. (1997). A respecification and extension of the DeLone and McLean model of IS success. Information Systems Research, 8(3), 240253. doi:10.1287/isre.8.3.240

Segars, A. H. \& Grover, V. (1993, December). Re-examining perceived ease of use and usefulness: a confirmatory factor analysis. MIS Quarterly, $17(4), 517-525$.

Simmhan, Y. L., Plale, B., \& Gannon, D. (2005). A survey of data provenance in e-science. ACM SIGMOD Record, $34(3), 36$.

Sivo, S. A., Saunders, C., Chang, Q., \& Jiang, J. J. (2006). How low should you go? low response rates and the validity of inference in IS questionnaire research. Journal of the Association for Information Systems, 7(6), $351-414$. 
Smith, S. M. \& Albaum, G. S. (2010). An introduction to marketing research. Qualtrics Labs Inc. Retrieved April 24, 2013, from http://www.qualtrics. com/university/researchsuite/ebooks

Smith, S. M. \& Albaum, G. S. (2012). Basic marketing research: volume 1. Qualtrics Labs Inc. Retrieved April 24, 2013, from http://www.qualtrics. com/university/researchsuite/ebooks

Smyth, Z. A. (2005). Implementing EDRM: has it provided the benefits expected? Records Management Journal, 15(3), 141.

Spencer, D. \& Warfel, T. (2004, April 7). Card sorting: a definitive guide. Retrieved 2013, from http:// boxesandarrows.com/card-sorting-adefinitive-guide/

Sprehe, J. T. (2005). The positive benefits of electronic records management in the context of enterprise content management. Government Information Quarterly, 22(2), 297-303. doi:10.1016/j.giq.2005.02.003

Sprehe, J. T. (2008, June). Exploring the information management side of RIM. Information Management Journal, 42(3), 62-67.

Sprehe, J. T. \& McClure, C. R. (2005, July). Lifting the burden. Information Management Journal, 39(4), 47. doi:Article

Steel, D. G. \& Holt, D. (1996, April 1). Analysing and adjusting aggregation effects: the ecological fallacy revisited. International Statistical Review / Revue Internationale de Statistique, 64(1), 39-60. doi:10.2307/1403423

Stein, E. W. (1995). Organizational memory: review of concepts and recommendations for management. International Journal of Information Management, 15(1), 17-32.

Stewart, C. J. \& Cash, W. B. (2008). Interviewing: principles and practices. Boston: McGraw-Hill Higher Education.

Straub Jr., D. W. \& Burton-Jones, A. (2007, April). Veni, vidi, vici: breaking the TAM logjam. Journal of the Association for Information Systems, $8(4), 224-229$.

Straub, D. W. (1989). Validating instruments in MIS research. MIS quarterly, 147-169. Retrieved April 26, 2013, from http://www.jstor.org/stable/ $10.2307 / 248922$

Straub, D. W., Limayem, M., \& Karahanna-Evaristo, E. (1995). Measuring system usage: implications for IS theory testing. Management Science, $1328-1342$.

Suhr, D. (2006). Exploratory or confirmatory factor analysis. Cary: SAS Institute. SUGI, 1-17. 
Svärd, P. (2013). Enterprise content management and the records continuum model as strategies for long-term preservation of digital information. Records Management Journal, 23(3), 159-176.

Svärd, P. (2014). The impact of information culture on information/records management: a case study of a municipality in Belgium. Records Management Journal, 24(1), 5-21.

Szajna, B. \& Scamell, R. W. (1993, December). The effects of information system user expectations on their performance and perceptions. MIS Quarterly, 17(4), 493-516.

Taylor, S. \& Todd, P. (1995a, December). Assessing IT usage: the role of prior experience. MIS Quarterly, 19(4), 561-570. doi:10.2307/249633

Taylor, S. \& Todd, P. (1995b). Understanding information technology usage: a test of competing models. Information Systems Research, 6(2), 144-176.

Tenenhaus, M., Vinzi, V. E., Chatelin, Y.-M., \& Lauro, C. (2005). PLS path modeling. Computational Statistics \&f Data Analysis, 48(1), 159-205. doi:10.1016/j.csda.2004.03.005

Thompson, R. L., Higgins, C. A., \& Howell, J. M. (1991, March). Personal computing: toward a conceptual model of utilization. MIS Quarterly, 15(1), 125-143.

Turner, M., Kitchenham, B., Brereton, P., Charters, S., \& Budgen, D. (2010). Does the technology acceptance model predict actual use? A systematic literature review. Information and Software Technology, 52(5), 463-479.

Upward, F. (1996, November). Structuring the records continuum part one: post-custodial principles and properties. Archives and Manuscripts, $24(2), 268-285$.

Upward, F. (1997, May). Structuring the records continuum part two: structuration theory and recordkeeping. Archives and Manuscripts, 25(1), $10-35$.

Upward, F. (2000). Modelling the continuum as paradigm shift in recordkeeping and archiving processes and beyond: a personal reflection. Records Management Journal, 10(3), 115-139.

Upward, F. (2005). The records continuum. In S. McKemmish, M. Piggott, B. Reed, \& F. Upward (Eds.), Archives: recordkeeping in society (pp. 197222). New South Wales: Centre for Information Studies, CSU.

Upward, F., Reed, B., Oliver, G., \& Evans, J. (2013, March 22). Recordkeeping informatics: re-figuring a discipline in crisis with a single minded approach. Records Management Journal, 23(1), 37-50. doi:10.1108/ 09565691311325013 
Van der Heijden, H. (2004). User acceptance of hedonic information systems. MIS Quarterly, 28(4), 695-704.

van Raaij, E. M. \& Schepers, J. (2008, April). The acceptance and use of a virtual learning environment in China. Computers \& Education, 50(3), 838-852. doi:10.1016/j.compedu.2006.09.001

Venkatesh, V. \& Bala, H. (2008). Technology acceptance model 3 and a research agenda on interventions. Decision Sciences, 39(2), 273-315. doi:10.1111/j.1540-5915.2008.00192.x

Venkatesh, V. \& Davis, F. D. (2000, February). A theoretical extension of the technology acceptance model: four longitudinal field studies. Management Science, 46(2), 186-204.

Venkatesh, V., Morris, M. G., Davis, G. B., \& Davis, F. D. (2003, September). User acceptance of information technology: toward a unified view. MIS Quarterly, 27(3), 425-478.

Venkatesh, V., Sykes, T., \& Zhang, X. (2011). 'Just what the doctor ordered': a revised UTAUT for EMR system adoption and use by doctors. (pp. 1-10). 2011 44th Hawaii international conference on system sciences (HICSS). Koloa, Kauai, Hawaii. doi:10.1109/HICSS.2011.1

Venkatesh, V., Thong, J., \& Xu, X. (2012). Consumer acceptance and use of information technology: extending the unified theory of acceptance and use of technology. MIS Quarterly, 36(1), 157-178.

Venkatesh, V.\& Zhang, X. (2010). Unified theory of acceptance and use of technology: U.S. vs. China. Journal of Global Information Technology Management, 13(1), 5-27.

Verhoeven, J. C., Heerwegh, D., \& De Wit, K. (2010, August). Information and communication technologies in the life of university freshmen: an analysis of change. Computers \& Education, 55(1), 53-66. doi:10.1016/ j.compedu.2009.12.002

Vinzi, V. E., Trinchera, L., \& Amato, S. (2010). PLS path modeling: from foundations to recent developments and open issues for model assessment and improvement. In Handbook of partial least squares (pp. 47-82). Springer: Handbooks of Computational Statistics.

Walsh, J. P. \& Ungson, G. R. (1991). Organizational memory. Academy of Management Review, 16(1), 57-91.

Wang, Y.-S., Wu, M.-C., \& Wang, H.-Y. (2009). Investigating the determinants and age and gender differences in the acceptance of mobile learning. British Journal of Educational Technology, 40(1), 92-118. doi:10.1111/ j.1467-8535.2007.00809.x 
Warshaw, P. R. (1980). A new model for predicting behavioral intentions: an alternative to Fishbein. Journal of Marketing Research, 17(2).

Weaver, G. R. \& Gioia, D. A. (1994). Paradigms lost: incommensurability vs structurationist inquiry. Organization Studies, 15(4), 565.

Wilkins, L., Holt, D., Swatman, P., \& Chan, E. (2007, June). Implementing information management strategically: an Australian EDRMS case study. In Merging and emerging technologies, processes and institutions. 20th Bled eMergence. Bled, Slovenia.

Wilkins, L., Swatman, P., \& Holt, D. (2009a, February 20). Achieved and tangible benefits: lessons learned from a landmark EDRMS implementation. Records Management Journal, 19(1), 37-53. doi:10.1108/ 09565690910937236

Wilkins, L., Swatman, P., \& Holt, D. (2009b, June 19). Adding value to enterprise-wide system integration: a new theoretical framework for assessing technology adoption outcomes. In Proceedings of the IADIS international conference e-commerce 2009 (pp. 53-60). IADIS multiconference on computer science and information systems 2009. Algarve, Portugal: IADIS Press.

Williams, D. (2005). EDRM implementation at the national weights and measures laboratory. Records Management Journal, $15(3), 158$.

Wilson, J. (1995). A framework for identifying the business benefits of electronic document management. Records Management Journal, 5(2), 9399. doi:10.1108/eb027081

Wood, J. R. \& Wood, L. E. (2008). Card sorting: current practices and beyond. Journal of Usability Studies, 4(1), 1-6. Retrieved February 18, 2013, from http://usabilityprofessionals.org/upa_publications/jus/ 2008november/JUS_Wood_Nov2008.pdf

Woods, L., Priest, H., \& Roberts, P. (2002). An overview of three different approaches to the interpretation of qualitative data. Part 2: Practical illustrations. Nurse Researcher, 10(1), 43.

Yuthas, K. \& Young, S. T. (1998). Material matters: assessing the effectiveness of materials management IS. Information \& Management, 33(3), 115124.

Zheng, Y. (2005). Information culture and development: chinese experience of e-health. In HICSS 2005 - Proceedings of the 38th Annual Hawaii International Conference on System Sciences (153a-153a). IEEE. 